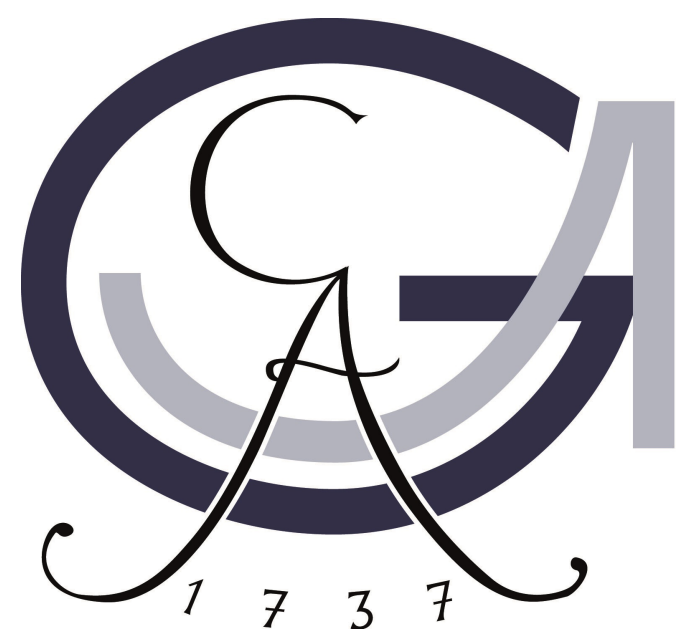

\title{
Variational Geometric Invariant Theory and Moduli of Quiver Sheaves
}

\section{Dissertation}

zur Erlangung des mathematisch-naturwissenschaftlichen Doktorgrades Doctor rerum naturalium

der Georg-August-Universität Göttingen

im Graduiertenkolleg 1493

der Georg-August-University School of Science (GAUSS)

vorgelegt von

Marcel Maslovarić

aus Langenfeld

Göttingen, 2017 


\section{Betreuungsausschuss}

Erstbetreuer: Jun.-Prof. Dr. Henrik Seppänen

Mathematisches Institut, Georg-August-Universität Göttingen Zweitbetreuer: Prof. Dr. Victor Pidstrygach

Mathematisches Institut, Georg-August-Universität Göttingen

\section{Mitglieder der Prüfungskommission}

Referent: Jun.-Prof. Dr. Henrik Seppänen

Mathematisches Institut, Georg-August-Universität Göttingen Koreferent: Prof. Dr. Victor Pidstrygach

Mathematisches Institut, Georg-August-Universität Göttingen

weitere Mitglieder der Prüfungskommission

Prof. Dr. Gert Lube

Institut für Numerische und Angewandte Mathematik, Georg-August-Universität Göttingen

Prof. Dr. Thomas Schick

Mathematisches Institut, Georg-August-Universität Göttingen Prof. Dr. Evelina Viada

Mathematisches Institut, Georg-August-Universität Göttingen Prof. Dr. Max Wardetzky

Institut für Numerische und Angewandte Mathematik, Georg-August-Universität Göttingen

Tag der mündlichen Prüfung

18. Januar 2018 



\begin{abstract}
We are concerned with two applications of GIT.

First, we prove that a geometric GIT quotient of an affine variety $X=$ $\operatorname{Spec}(A)$ by a reductive group $G$, where $A$ is an almost factorial domain, is a Mori dream space, regardless of the codimension of the unstable locus. This includes an explicit description of the Picard number, the pseudoeffective cone, and the Mori chambers in terms of GIT.

We apply the results to quiver moduli to show that they are Mori dream spaces if the quiver contains no oriented cycles, and if stability and semistability coincide. We give a formula for the Picard number in quiver terms. As a second application, we prove that geometric quotients of Mori dream spaces are Mori dream spaces as well, which again includes a description of the Picard number and the Mori chambers. Some examples are given to illustrate the results.

The second instance where we use GIT, is the construction and variation of moduli spaces of quiver sheaves.

To that end, we generalize the notion of multi-Gieseker semistability for coherent sheaves, introduced by Greb, Ross, and Toma, to quiver sheaves for a quiver $Q$. We construct coarse moduli spaces for semistable quiver sheaves using a functorial method that realizes these as subschemes of moduli spaces of representations of a twisted quiver, depending on $Q$, with relations. We also show the projectivity of the moduli space in the case when $Q$ has no oriented cycles. Further, we construct moduli spaces of quiver sheaves which satisfy a given set of relations as closed subvarieties.

Finally, we investigate the parameter dependence of the moduli.
\end{abstract}




\section{Acknowledgments}

My deepest gratitude goes to my thesis advisor Henrik Seppänen, for his patient support and many fruitful discussions, both about mathematics and on general nonsense. I also thank the other members of our working group for a stimulating atmosphere, and in particular Georg Merz, my fellow inmate of room 2.111 .

Furthermore, I thank the people I got to know at the institute, during conferences and as a guest.

Last, but certainly not least, I thank my family and friends for their love and support throughout this endeavor.

Funding acknowledgment: This thesis was supported by the German Research Foundation (DFG) through the Research Training Group 1493 "Mathematical structures in modern quantum physics" at the University of Göttingen. 


\section{Contents}

Introduction $\quad 5$

$\begin{array}{ll}\text { Chapter 1. Preliminaries } & 17\end{array}$

1.1. Geometric Invariant Theory 18

1.1.1. Good and geometric quotients 18

$\begin{array}{ll}\text { 1.1.2. GIT quotients } & 19\end{array}$

1.1.3. Variational GIT 21

1.2. Quivers and their representations 25

1.2.1. The category of representations 26

1.2.2. Stability conditions 31

1.2.3. Moduli spaces 32

1.3. Rational maps and divisors 34

1.3.1. Divisors 34

1.3.2. Rational contractions 37

1.4. Mori dream spaces 38

1.4.1. Characterization via cones of divisors 38

1.4.2. GIT quotients and the Cox ring 40

1.5. Almost factorial domains 42

Chapter 2. Mori dream spaces $\quad 45$

2.1. Quotients of spectra of AFDs 45

2.1.1. Divisors under the group action 45

2.1.2. Divisors under descent 49

2.1.3. The main theorem 53

2.2. Quotients of Mori dream spaces 58

2.3. Application to quiver moduli 62

2.4. Examples 64

2.4.1. Affine prequotients 64

2.4.2. Quiver moduli examples $\quad 67$

2.4.3. Quotients of Mori dream spaces 71

Chapter 3. Moduli spaces of quiver sheaves 73

3.1. Quiver sheaves 73

3.1.1. Basic notions $\quad 73$

3.1.2. Stability conditions $\quad 75$

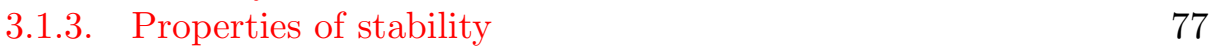

3.2. The embedding functor $\quad 82$

3.2.1. The twisted quiver $\quad 82$

3.2.2. The functor $\quad 85$

3.3. Stability under embedding $\quad 89$

3.3.1. The Le Potier-Simpson theorem 89 
3.3.2. Semistability under embedding

3.3.3. $S$-equivalence under embedding

3.4. Construction of the moduli space

3.5. Projectivity of the moduli space

3.5.1. Extensions

106

3.5.2. Langton's theorem

108

3.6. Variation of stability conditions

117

3.6.1. Walls and chambers

3.6.2. Variation of moduli spaces

3.7. Symmetric stability conditions

3.8. Quiver Quot-Schemes

Bibliography

Anhänge

Erklärung gemäß $§ 8.2$

133

Lebenslauf 


\section{Introduction}

In his talk [Mum63], Mumford outlined a strategy for building varieties, or schemes, which parameterize algebro-geometric objects - like, the vector bundles on a curve, or 0-cycles on a variety. Later, he developed this idea into Geometric Invariant Theory (GIT), as explained in his famous book [MFK94].

The general strategy is to write the objects under consideration as a variety $X$, together with the action of a group $G$, which encodes isomorphisms of the objects. To parameterize the isomorphism classes, we should then try to find a variety structure on the set of orbits.

Though in general, it is not possible to find a scheme structure on the whole set of orbits: in some sense, this set is too large. But on a subset consisting of so called stable orbits, or on semistable orbits, a construction is possible. This implies that for classification problems of algebro-geometric objects, we have to find suitable notions of stable and semistable objects as well.

From here, two natural directions of further development emerge. First, one could try to apply Mumford's strategy in order to construct moduli spaces of other interesting objects. Another natural question is to describe properties of the spaces of orbits, also called GIT quotients, in terms of properties of the action on $X$.

\section{Mori dream spaces}

One example of the second line of thought is the notion of a Mori dream space, as introduced by $\mathrm{Hu}$ and Keel in [HK00].

By construction, Mori dream spaces are varieties which are well-behaved with respect to Mori's minimal model program. They are also closely related to Variational Geometric Invariant Theory.

Examples of Mori dream spaces include $\mathbb{P}^{n}$, Grassmannians, quiver moduli, toric varieties, and Fano varieties.

The results of this thesis which are concerned with Mori dream spaces may be found in Chapter 2. Also, they are available as the preprint [Mas15].

\section{Mori dream spaces and VGIT.}

Suppose that a reductive group $G$ acts on a quasiprojective variety $X$. The construction of a GIT quotient starts with the choice of an ample line bundle

$$
E \rightarrow X \text {, }
$$


together with a lift of the group action, a so called $G$-line bundle. To such a choice, GIT associates the semistable locus

$$
X^{E-\text { sst }}=\bigcup_{s} X \backslash N(s),
$$

where the union is taken over all $s \in H^{0}(X, m E)^{G}$ for all multiples $m>0$. This is an open subset, and the central result of GIT is the existence of a quotient

$$
q_{E}: X^{E-\text { sst }} \rightarrow Y_{E} .
$$

A simple, yet important, example of a $G$-line bundle is $L_{\chi}$, given by the trivial line bundle

$$
L \rightarrow X,
$$

where the lift of the group action is induced by a character $\chi$ of the group $G$.

Evidently, the construction of the GIT quotient depends on the choice of the $G$-line bundle $E$. Variational Geometric Invariant Theory (VGIT), as developed by [BP89],[DH98],[Hu92],[Th96],[Hal04], provides a systematic study of this dependence.

The $G$-line bundles which are ample as line bundles and have non-empty semistable locus form a rational polyhedral cone

$$
C^{G}(X) \subset \mathrm{NS}^{G}(X)_{\mathbb{R}}
$$

in the space of all $G$-line bundles. This cone is called the $G$-ample cone. Moreover, the $G$-ample cone is divided into finitely many full-dimensional and rational polyhedral subcones, the so called GIT chambers. The hyperplanes diving the GIT chambers are called GIT walls.

Within a chamber, the semistable locus does not depend on the concrete choice of the $G$-line bundle. Consequently, the quotient does not depend on it as well.

Suppose that we have two neighboring GIT chambers $C_{+}$and $C_{-}$, separated by a wall $H$. A line segment connecting interior points $E_{+} \in C_{+}$and $E_{-} \in C_{-}$of the chambers intersects $H$ in a $G$-line bundle $E_{0}$. In good cases, the corresponding quotients are related by a so called Thaddeus flip

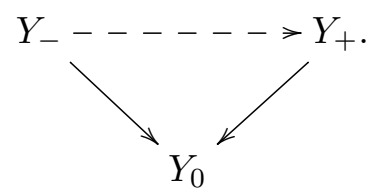

Hence, VGIT influences the birational geometry of the quotients. We remark, that the construction of rational maps between the quotients is not restricted to this case. Under some mild conditions, it is possible to map from the quotient associated to any point in the interior of the $G$-ample cone to any other quotient.

On a Mori dream space $Y$, the cone of pseudo-effective divisors

$$
\overline{\operatorname{Eff}}(Y) \subset N^{1}(Y)_{\mathbb{R}}
$$


admits a similar decomposition into finitely many full-dimensional and rational polyhedral cones with disjoint interior, the so called Mori chambers. They have the property that for all divisors in the interior of one fixed Mori chamber, the associated contractions

$$
f_{D}: Y \rightarrow Y_{D}
$$

are well-defined, and are equivalent in some suitable sense. The equivalence used here is the notion of Mori equivalence, also introduced by $\mathrm{Hu}$ and Keel.

If the variety and group action satisfy some good conditons, a GIT quotient translates between these two chamber structures.

Assumption 1. Among some technical conditions, $\mathrm{Hu}$ and Keel consider the action of a reductive group $G$ on an affine variety $X$, and the choice of a character $\chi_{0}$ of $G$, such that the following conditions hold.

(1) $X$ is normal and $\mathrm{Cl}(X)_{\mathbb{Q}}=0$.

(2) $\chi_{0}$ is contained in the interior of a GIT chamber, and

$$
\operatorname{codim}\left(X \backslash X^{\chi-\text { sst }}\right) \geq 2 \text {. }
$$

Here, the first condition implies that all $G$-line bundles are, up to multiples, of the form $L_{\chi}$ for a character $\chi$. In that sense, the GIT chamber structure for $G$-line bundles reduces to a chamber structure in the space $\chi(G)_{\mathbb{R}}$ of characters, explaining the second condition.

In the following, we will refer to the inequality in the second assertion as the codimension condition on the unstable locus.

$\mathrm{Hu}$ and Keel then show that the GIT quotient $Y=Y_{\chi_{0}}$, associated to $\chi_{0}$, is a Mori dream space, closely related to the VGIT of the group action on $X$.

Theorem 1. Under the Assumption above, the following assertions hold.

(1) $Y$ is a Mori dream space.

(2) Descent of $G$-line bundles induces an isomorphism

$$
\psi: \chi(G)_{\mathbb{Q}} \rightarrow \operatorname{Pic}(Y)_{\mathbb{Q}} .
$$

(3) The map $\psi$ identifies the $G$-ample cone with the pseudo-effective cone, and the GIT chambers with the Mori chambers.

(4) For any divisor $D$, the associated map

$$
f_{D}: Y \rightarrow Y_{D}
$$

is equivalent to the rational map induced by VGIT for a character $\chi$ such that $\psi(\chi)=D$.

Conversely, to a Mori dream space $Y, \mathrm{Hu}$ and Keel associate an affine variety $X$, such that $Y$ is given as a GIT quotient satisfying the conditions imposed above.

This affine variety is the spectrum of a so called Cox ring

$$
\operatorname{Cox}(Y)=\bigoplus_{a \in \mathbb{Z}^{r}} H^{0}\left(Y, \mathcal{O}\left(a_{1} D_{1}+\ldots+a_{r} D_{r}\right)\right),
$$


where $D_{1}, \ldots, D_{r}$ are Cartier divisors on $Y$ such that

$$
\mathcal{O}\left(D_{1}\right), \ldots, \mathcal{O}\left(D_{r}\right)
$$

forms a basis of the vector space $\operatorname{Pic}(Y)_{\mathbb{Q}}$. The group action is that of the torus $T=T^{r}$, action via the multi-grading of the Cox ring.

To ensure that this construction is well-defined, $\mathrm{Hu}$ and Keel show that if $Y$ is a Mori dream space, $\operatorname{Cox}(Y)$ is finitely generated as a $k$-algebra.

\section{Unstable components in codimension one.}

In applications of $\mathrm{Hu}$ and Keel's results, the codimension condition on the unstable locus seems to be a technical hurdle.

For example, in [J11], Jow even considers a Mori dream space $Y$ such that the unstable locus in the spectrum $X$ of the Cox ring is of codimension at least three. This requirement is guided by his desire to construct a hypersurface $Y^{\prime} \subset Y$ which is itself a Mori dream space. Indeed, his stronger codimension assumption ensures, that for a hypersurface in $X$, the unstable locus is of codimension at least two.

Another example is the work [Cr11] of Craw, where he wants to consider a certain class of quiver moduli as Mori dream spaces. The need to ensure the codimension condition restricts him to a rather limited special case.

On the other hand, in [Ba11], Bäker proved, that a quotient of a variety with finitely generated Cox ring also has finitely generated Cox ring in general, posing no condition on the unstable locus at all. But, his work does not allow insight into the quantitative data, like the Mori chambers. Even the Picard rank remains unknown.

It thus seems desirable to obtain a version of $\mathrm{Hu}$ and Keel's theorem about GIT quotients, which does not need the codimension condition. This was achieved by the author in [Mas15], and is outlined in Section 2.1.

Consider a situation as in the Assumption of $\mathrm{Hu}$ and Keel, but where the unstable locus is potentially of codimension one. We have an induced action of the group $G$ on the irreducible components

$$
\mathcal{Z}=\left\{Z_{1}, \ldots, Z_{s}\right\}
$$

of the unstable locus which are of codimension one. To each orbit

$$
B_{i} \in \mathcal{Z} / G=\left\{B_{1}, \ldots, B_{r}\right\},
$$

we can associate a regular function $f_{i}$, such that

$$
N\left(f_{i}\right)=\bigcup_{Z \in B_{i}} Z,
$$

and such that $f_{i}$ is a semiinvariant with respect to a character $\chi_{i}$. The descend of line bundles $\psi$ then has a kernel

$$
0 \rightarrow \mathbb{Q}^{r} \rightarrow \chi(G)_{\mathbb{Q}} \stackrel{\psi}{\rightarrow} \operatorname{Pic}(Y)_{\mathbb{Q}} \rightarrow 0,
$$

which is spanned by the characters $\chi_{i}$.

As we can show, the quotient is still a Mori dream space, but not all GIT 
chambers correspond to a Mori chamber. By definition, a GIT chamber $C \subset \chi(G)_{\mathbb{Q}}$ is called stable with respect to $\chi_{0}$, if

$$
\chi_{i}+C \subset C
$$

for all the characters $\chi_{i}$ associated to the orbits in $\mathcal{Z}$. Our main result is now the following.

Theorem 2. Under the Assumption of $\mathrm{Hu}$ and Keel, but where the unstable locus is potentially of codimension one, the following assertions hold.

(1) $Y$ is a Mori dream space.

(2) Descent of $G$-line bundles induces a surjection

$$
\psi: \chi(G)_{\mathbb{Q}} \rightarrow \operatorname{Pic}(Y)_{\mathbb{Q}},
$$

such that the kernel is spanned by the, linearly independent, characters $\chi_{i}$ associated to the orbits in $\mathcal{Z}$.

(3) The union of the images of the stable GIT chambers under $\psi$ is the pseudo-effective cone of $Y$, and the stable GIT chambers are identified with the Mori chambers.

(4) For any divisor $D$, the associated map

$$
f_{D}: Y \rightarrow Y_{D}
$$

is equivalent to the rational map induced by VGIT for a character $\chi$ such that $\psi(\chi)=D$, and such that $\chi$ is contained in the closure of a stable GIT chamber.

\section{Applications of the Theorem.}

We provide two applications of our main result. The application to quiver moduli is discussed in Section 2.3, and the application to quotients of Mori dream spaces may be found in Section 2.2.

Quiver moduli. In the language of representation theory of algebras, directed graphs are typically called quivers. Moduli spaces for the representations of a quiver, or simply quiver moduli, were constructed by King, employing Mumford's general strategy (cf. [K94]). The GIT quotient he used satisfies the Assumption of $\mathrm{Hu}$ and Keel, except for the codimension condition on the unstable locus.

Given a quiver $Q$, consisting of a set of vertices $Q_{0}$ and arrows $\alpha: i \rightarrow j$, King considered the representation variety

$$
R_{d}(Q)=\bigoplus_{\alpha: i \rightarrow j} \operatorname{Hom}\left(k^{d_{i}}, k^{d_{j}}\right),
$$

where $d$ is a fixed dimension vector, assigning to each vertex $i \in Q_{0}$ a dimension $d_{i}$. Simultaneous base change induces a natural action of the group

$$
G_{d}=\prod_{i \in Q_{0}} \mathrm{GL}\left(d_{i}\right)
$$

on this variety. King then showed that the isomorphism classes of representations of $Q$, with fixed dimension vector $d$, correspond to the orbits of this 
action. Furthermore, he translated GIT stability on $R_{d}(Q)$ to a stability condition for representations. Hence, the GIT quotients parameterize such representations, forming a moduli space.

It is not true in general that the unstable loci are of codimension two, so that the results of $\mathrm{Hu}$ and Keel can not be applied directly. However, our results show that quiver moduli are Mori dream spaces even if this is not the case. Since the GIT behavior of the representation variety admits an, at least partial, description via combinatorics, the same is thus true for the birational geometry of quiver moduli. We build upon this philosophy in some of the examples in Section 2.4.

Quotients of Mori dream spaces. As a second application, we were concerned with quotients of a Mori dream space $Y$. Suppose that a connected and reductive group $G$ acts on $Y$, such that there exists a quotient

$$
q^{\prime}: V \rightarrow Z
$$

for a $G$-invariant and open subset $V \subset Y$. Using the Cox ring description of $Y$, we were able to express $Z$ as a quotient

$$
q: U \rightarrow Z
$$

for an open and $T \times G$-invariant subset $U \subset X=\operatorname{Spec}(\operatorname{Cox}(Y))$. Here, $T$ is the torus used in the Cox ring description of a Mori dream space, and the quotient $q$ is taken with respect to the lifted action of $T \times G$. By comparing these two actions, and applying our main result, we are able to prove the following.

Theorem 3 . The variety $Z$ is a Mori dream space, and

$$
\text { rk } \operatorname{Pic}(Z)=\operatorname{rk} \operatorname{Pic}(Y)+\operatorname{rk} \chi(G)-|\mathcal{Z}(V)|,
$$

where $\mathcal{Z}(V)$ is the set of irreducible components of the complement of $V$ in codimension one. The Mori chamber structure of $Z$ is determined by the stable GIT chamber structure of $T \times G$ on $X$.

\section{Moduli spaces of quiver sheaves}

As a second project, we applied GIT to construct moduli spaces of multiGieseker semistable quiver sheaves, following the general strategy of Mumford. Furthermore, we establish a theory of variation for these moduli spaces, which includes a variation result for the usual notion of stability for quiver sheaves.

In Chapter 3, the reader may find the detailed results of this thesis which are concerned with quiver sheaves. Additionally, many results are available in the preprint [MS17]. This is joint work with Henrik Seppänen. 


\section{Gieseker-stability of sheaves.}

The vector bundles on a curve were one of the earliest examples of a class of algebro-geometric objects which can be parameterized using Mumford's strategy. The work of Gieseker and Maruyama (cf. [Gi77],[Mar77]), extended this classification to the case of torsion-free sheaves on arbitrary smooth and projective varieties. Finally, Simpson gave a further extension to the case of pure sheaves (cf. [Si94]).

We briefly sketch Simpson's approach.

A purely $d$-dimensional sheaf $E$ is Gieseker-semistable, if for all non-trivial subsheaves $F \subset E$ we have an inequality

$$
\frac{P_{F}^{L}}{\alpha_{d}^{L}(F)} \leq \frac{P_{E}^{L}}{\alpha_{d}^{L}(E)}
$$

where $P_{F}^{L}$ and $P_{E}^{L}$ are the Hilbert polynomials with respect to some fixed ample line bundle $L$ on $X$, and $\alpha_{d}^{L}(F)$ as well as $\alpha_{d}^{L}(E)$ are their leading coefficients.

Since the family of semistable sheaves with fixed Hilbert polynomial $P$ is bounded, such sheaves are $n$-regular for sufficiently large $n$. Further, they can be embedded as points in a Grassmannian scheme

$$
\left[V \otimes H \rightarrow H^{0}(E(m))\right] \in \operatorname{Grass}^{P(m)}(V \otimes H),
$$

where $H=H^{0}(\mathcal{O}(m-n))$, and $V \simeq H^{0}(E(n))$ is a fixed vector space among the $h^{0}(E(n))$-dimensional ones, and $m \gg n \gg 0$. Different choices of the isomorphism $V \simeq H^{0}(E(n))$ correspond to the action of $\mathrm{GL}(V)$ on the Grassmannian scheme. One can then show, that the semistable sheaves correspond to GIT semistable points in the Grassmannian, and that the GIT quotient of the image of the embedding parameterizes $S$-equivalence classes of semistable sheaves.

This approach was later reformulated by Álvarez-Cónsul and King in a functorial way (cf. [ACK07]), where they build upon the fact that Grassmannians are quiver moduli.

In some analogy to the case of VGIT, the set of Gieseker-semistable sheaves, and hence their moduli space, depends on the choice of the ample line bundle $L$. The behavior under variation of $L$ bears some similarity to VGIT. Namely, the ample cone of $X$ is divided into chambers by hypersurfaces, such that stability does not depend on the explicit choice of $L$ within some fixed chamber. However, in contrast to the case of VGIT, the walls are generally of higher degree, and need not contain the class of an honest line bundle, potentially consisting only of formal real powers of line bundles (consider [Sch00] for an example). Hence, for such a wall, a birational map

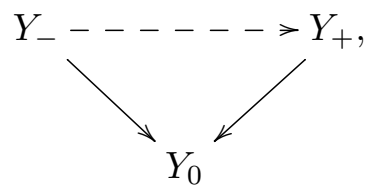


which is the analogue of a Thaddeus flip, can not be constructed. A notable exception is the case of a surface (eg. consider [MW97]). Here, the walls are rational hyperplanes, so that analogues of Thaddeus flips exist, and the moduli spaces are generically birational.

\section{Multi-Gieseker-stability of sheaves.}

In [GRT16], Greb, Ross and Toma approached this problem from a different direction. Instead of varying the line bundle $L$ directly, they essentially consider linear combinations of the stability conditions for different line bundles.

Let $\left(L_{1}, \ldots, L_{N}\right)$ denote a tuple of ample line bundles on $X$, which we think of as fixed, and choose a tuple $\sigma \in \mathbb{R}_{\geq 0}^{N}$. Then, a purely $d$-dimensional sheaf $E$ is called multi-Gieseker semistable if for all non-trivial subsheaves $F \subset E$ we have an inequality

$$
\frac{\sum_{j=1}^{N} \sigma_{j} P_{F}^{L_{j}}}{\sum_{j=1}^{N} \sigma_{j} \alpha_{d}^{L_{j}}(F)} \leq \frac{\sum_{j=1}^{N} \sigma_{j} P_{E}^{L_{j}}}{\sum_{j=1}^{N} \sigma_{j} \alpha_{d}^{L_{j}}(E)} .
$$

Clearly, for $\sigma=e_{j}$, this recovers the notion of usual Gieseker-semistability with respect to the ample line bundle $L_{j}$. In that sense, the problem of variation of $L$ is translated into a problem of variation of $\sigma$ within the space $\mathbb{R}_{\geq 0}^{N}$.

For the case of torsion-free sheaves on an integral and projective scheme, Greb, Ross and Toma showed that there is a decomposition of $\mathbb{R}_{\geq 0}^{N}$ into chambers by finitely many rational hyperplanes, which encode the equivalence of multi-Gieseker semistability.

Moreover, subject to a boundedness condition, and after fixing the Hilbert polynomials, they constructed moduli spaces of multi-Gieseker semistable sheaves, by building upon the functorial approach of Álvarez-Cónsul and King. In their work, they construct an embedding functor

$$
\operatorname{Hom}(T, *): \operatorname{Coh}(X) \rightarrow Q^{\prime}-\operatorname{rep},
$$

from the category of sufficiently regular sheaves, to the category of representations of an auxiliary quiver $Q^{\prime}$, which is schematically given as follows

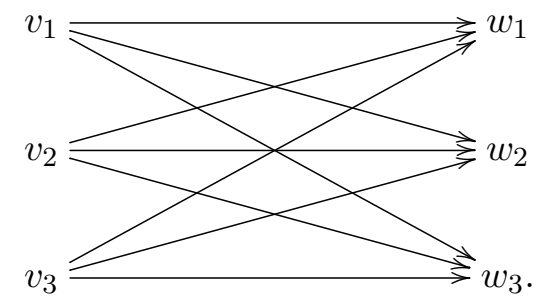

Each row of this quiver corresponds to the functor as introduced by ÁlvarezCónsul and King, further corresponding to one of the ample line bundles $L_{j}$. Consequently, there is a parameter space for such sheaves which is embedded into the representation variety $R_{d}\left(Q^{\prime}\right)$.

By an argument in the spirit of Le Potier and Simpson, they further showed that the multi-Gieseker semistable sheaves correspond to GIT-semistable 
points. Hence, the moduli space of semistable sheaves $M^{\sigma-\text { sst }}(X)$ is embedded into the moduli space of semistable quiver representations.

Furthermore, by invoking an argument of Langton, they showed that this moduli space is projective. Together with the result about the chamber structure indicated above, this enabled them to establish a variation result. More precisely, they show that for two stability conditions $\sigma_{1}$ and $\sigma_{2}$, the moduli spaces $M^{\sigma_{1}-\text { sst }}(X)$ and $M^{\sigma_{2}-\text { sst }}(X)$ are related by a sequence of Thaddeus flips

$$
M^{\sigma_{1}-\mathrm{sst}}(X) \rightarrow Y_{1} \rightarrow Y_{2} \rightarrow \ldots \rightarrow M^{\sigma_{2}-\mathrm{sst}}(X),
$$

and are thus birational. Here, the Thaddeus flips, and the varieties $Y_{i}$, are given by VGIT of a suitable variety $Z \subset R_{d}\left(Q^{\prime}\right)$ which encodes the image of the embedding functor $\operatorname{Hom}(T, *)$.

\section{The case of Quiver sheaves.}

In [GRT16], Greb, Ross and Toma raised the question whether their approach can be extended to quiver sheaves. Indeed, we are able to show that this is possible, as outlined in Chapter 3. Most of the results may also be found in the preprint [MS17].

Quiver sheaves are representations of a quiver $Q$ in the category of coherent sheaves on some scheme $X$. The prototypical example is that of a morphism of sheaves

$$
F \rightarrow G
$$

For a general quiver $Q$, a quiver sheaf $\mathcal{E}$ consists of sheaves $\mathcal{E}_{i}$ at the vertices, and morphisms of sheaves $\mathcal{E}_{\alpha}: \mathcal{E}_{i} \rightarrow \mathcal{E}_{j}$ attached to the arrows.

Stability conditions. We also fix a tuple $\left(L_{1}, \ldots, L_{N}\right)$ of ample line bundles, and consider stability conditions $\sigma \in \mathbb{R}_{>0}^{Q_{0} \times N}$. We then say that a purely $d$-dimensional quiver sheaf $\mathcal{E}$ is multi-Gieseker semistable, if for all non-trivial quiver subsheaves $\mathcal{F} \subset \mathcal{E}$ the inequality

$$
\frac{\sum_{i \in Q_{0}} \sum_{j=1}^{N} \sigma_{i j} P_{\mathcal{F}_{i}}^{L_{j}}}{\sum_{i \in Q_{0}} \sum_{j=1}^{N} \sigma_{i j} \alpha_{d}^{L_{j}}\left(\mathcal{F}_{i}\right)} \leq \frac{\sum_{i \in Q_{0}} \sum_{j=1}^{N} \sigma_{i j} P_{\mathcal{E}_{i}}^{L_{j}}}{\sum_{i \in Q_{0}} \sum_{j=1}^{N} \sigma_{i j} \alpha_{d}^{L_{j}}\left(\mathcal{E}_{i}\right)}
$$

holds. This notion reduces to that of usual Gieseker-semistability for quiver sheaves with respect to $L_{k}$ if we choose $\sigma_{i j}=\delta_{j k}$, and to the multi-Gieseker semistability for sheaves in the case that $Q$ consists of a single vertex, where quiver sheaves are simply ordinary sheaves.

The parameter space of possible stability conditions admits a decomposition respecting the change of stability as well.

TheOREM 4. The space $\mathbb{R}_{>0}^{Q_{0} \times N}$ is divided into chambers by finitely many hypersurfaces, such that the notion of stability is unchanged as we vary $\sigma$ within some chamber. 
These hypersurfaces need not be hyperplanes, but are of degree two in general, so that we can not ensure the existence of rational points on the walls.

Symmetric stability condition. However, for the special case of a symmetric stability condition $\sigma$, ie. such that the value of $\sigma_{i j}$ is independent from $i \in Q_{0}$, and torsion-free quiver sheaves, we are able to prove that the walls are indeed rational hyperplanes.

Another technical condition is the boundedness of the family of semistable quiver sheaves, which might possibly not hold in general. In the case of a symmetric stability condition $\sigma$, we show that the family of $\sigma$-semistable quiver sheaves is bounded if and only if the family of $\hat{\sigma}$-semistable sheaves is bounded. Here, $\hat{\sigma}$ is the multi-Gieseker stability condition for sheaves defined by the symmetry of $\sigma$. Hence, we can translate the main boundedness result of [GRT16] to the case of quiver sheaves.

Theorem 5. Suppose that one of the following conditions holds.

(1) The Picard number of $X$ is at most two.

(2) The dimension of $X$ is at most three.

(3) The ranks of the sheaves under consideration are at most two.

Then, the family of quiver sheaves which are semistable with respect to any symmetric stability condition $\sigma \in \mathbb{R}_{\geq 0}^{Q_{0} \times N}$, is bounded.

Hence, at least for symmetric stability conditions in this special case, all of our technical assumptions are satisfied.

Construction of the moduli space. Our construction of the moduli spaces of quiver sheaves further exploits the functorial approach of ÁlvarezCónsul and King. We consider a twisted quiver $Q\left(Q^{\prime}\right)$, essentially given by replacing each vertex of $Q$ with a copy of Greb, Ross and Toma's auxiliary quiver $Q^{\prime}$. Arrows $\alpha: i \rightarrow j$ in $Q$ are split up to arrows between the corresponding vertices in the copies of $Q^{\prime}$, attached to the vertices $i$ and $j$. Schematically, $Q\left(Q^{\prime}\right)$ thus looks like

$$
Q^{\prime} \rightleftharpoons Q^{\prime} \text {. }
$$

We additionally need relations $I^{\prime}$ on this quiver, to ensure that the morphisms attached to the split up arrows are compatible. On the technical side, this made it necessary to introduce an extension of King's construction of quiver moduli spaces to the case of labeled representations with relations.

The embedding functor $\operatorname{Hom}(T, *)$ of [GRT16] easily extends to a functor from the category of quiver sheaves on $X$ to the category of representations of $Q$ in the category of representations of $Q^{\prime}$, and the latter can be identified with representations of $Q\left(Q^{\prime}\right)$ satisfying the relations sketched above. Our embedding functor thus reads as

$$
\operatorname{Hom}(T, *): Q-\operatorname{Coh}(X) \rightarrow\left(Q\left(Q^{\prime}\right), H, I^{\prime}\right)-\text { rep. }
$$

Moreover, by extending the argument of Le Potier and Simpson even further, we manage to show that this embedding preserves stability, in the sense that $\operatorname{Hom}(T, \mathcal{E})$ is semistable for a suitable stability condition of vector space 
representations, if and only if $\mathcal{E}$ is multi-Gieseker semistable. Hence, we are able to form the GIT quotient of the image of the embedding functor.

Theorem 6. The moduli space $M^{\sigma-s s t}(X, Q)$ of multi-Gieseker semistable quiver sheaves can be constructed as the restriction of the GIT quotient which produces the moduli space of quiver representations of $Q\left(Q^{\prime}\right)$.

Variation of the moduli space. If $Q$ does not contain oriented cycles, we are able to show that the moduli space we constructed is projective. In view of the fact that moduli spaces of quiver representations are projective if and only if the quiver does not contain oriented cycles, this condition appears to be necessary. Combining this projectivity result with the description of the chamber structure, allows us to prove a variation result in the same spirit as that of Greb, Ross and Toma. That is, as we change $\sigma$, the moduli spaces are related by sequences of Thaddeus flips

$$
M^{\sigma_{1}-\text { sst }}(X, Q) \rightarrow Y_{1} \rightarrow Y_{2} \rightarrow \ldots \rightarrow M^{\sigma_{2}-\text { sst }}(X, Q),
$$

again given by VGIT of a suitable variety $Z \subset R_{d}\left(Q\left(Q^{\prime}\right), H, I^{\prime}\right)$, and are thus birational.

Relations on $Q$. In a further refinement, we succeed in incorporating relations $I$ on $Q$ into our results. For example, this makes it possible to consider moduli spaces of sheaves $E$, together with a nilpotent, or idempotent, endomorphism $\phi: E \rightarrow E$. Another example is that of a commutating square.

Again, the treatment of relations was greatly facilitated by the functorial nature of the construction. Namely, we can translate the relations $I$ on $Q$ to a set of relations $I^{\prime}(I)$ on $Q\left(Q^{\prime}\right)$, such that if $\mathcal{E}$ satisfies the relations $I$, $\operatorname{Hom}(T, \mathcal{E})$ satisfies the relations $I^{\prime}(I)$. Then, by following the program of construction similar to the one outlined above, we obtain the moduli space of quiver sheaves which satisfy the relations $I$, using the GIT construction of the quiver moduli.

TheOrem 7. The moduli space of multi-Gieseker semistable quiver sheaves which satisfy the relations $I$ is given as a closed subvariety

$$
M^{\sigma-\text { sst }}(X, Q, I) \subset M^{\sigma-\text { sst }}(X, Q) .
$$

If $Q$ does not contain oriented cycles, this moduli space is projective, and the same variation results as in the case without relations hold. 



\section{CHAPTER 1}

\section{Preliminaries}

In this chapter, we collect basic notions and results about the topics discussed in this thesis.

The discussion about Geometric Invariant Theory, rational maps and divisors contains almost no novel results, so we omit proofs here. For almost factorial domains, we review the results from Storch's PhD thesis [St67], and discuss the uniqueness of primary decompositions. The subsection about moduli of representations of quivers is fairly detailed, and contains sketches of proofs. Mainly, this is to justify our use of relations in the labeled case.

Before discussing the special topics, we give some general conventions.

Unless indicated otherwise, we work with varieties and schemes over an algebraically closed field $k$, and varieties are assumed to be irreducible. We restrict ourselves to characteristic zero.

Whenever we have a $\mathbb{Z}$-module $A$ we denote by

$$
A_{K}=A \otimes_{\mathbb{Z}} K
$$

the change of scalars, where $K \in\{\mathbb{Z}, \mathbb{Q}, \mathbb{R}\}$. Of course, a similar convention holds for $\mathbb{Q}$-vector spaces.

For a variety $X$ we denote

$$
E(X)=\mathcal{O}(X)^{*} / k^{*} .
$$

This is a free and finitely generated group (consider [KKV89], Proposition $1.3)$. If an algebraic group $G$ acts on $X$, there is an induced action of $G$ on $E(X)$.

Finally, we remark that over normal varieties we have an analogue of Hartogs' lemma (see Theorem 6.45 in [GW10]).

Lemma 1.0.1. Let $U \subset X$ denote an open subset in a normal variety such that $X \backslash U$ is of codimension greater or equal to 2. Then the restriction map

$$
\mathcal{O}(X) \rightarrow \mathcal{O}(U)
$$

is an isomorphism. 


\subsection{Geometric Invariant Theory}

We recollect basic definitions of Geometric Invariant Theory (GIT), mainly to fix notation. For the relevant aspects of VGIT we refer to [DH98] or [Th96], or to [Hal04] for the transfer to the affine case. For GIT itself, we refer to [MFK94].

\subsubsection{Good and geometric quotients.}

Let an algebraic group $G$ act on some variety $X$.

A naive hope is that the set $X / G$ of orbits canonically admits the structure of an algebraic variety such that the quotient map is a morphism of varieties. But this is not possible in general.

Assume that there was some quotient

$$
p: X \rightarrow X / G,
$$

which is a morphism of varieties. The fiber of a closed point $x \in X / G$ should be an orbit

$$
\mathcal{O}=p^{-1}(x),
$$

which must be closed by the continuity of $p$. Morally, quotients can thus only see closed orbits. But in general, orbits are not closed.

It turns out that good quotients are a useful approximation to the properties we desire.

Definition 1.1.1. Let an algebraic group $G$ act on a variety $X$. Then a morphism

$$
q: X \rightarrow Y
$$

is called a good quotient if the following holds.

(1) $q$ is surjective and invariant under the group action.

(2) For an affine open subset $U \subset Y$ the inverse image $q^{-1}(U) \subset X$ is affine and pullback of regular functions induces an isomorphism

$$
\mathcal{O}(U) \simeq \mathcal{O}\left(q^{-1}(U)\right)^{G} .
$$

If additionally, each fiber consists of exactly one orbit, we say that $q$ is a geometric quotient.

A good quotient $q: X \rightarrow Y$ is also a categorical quotient. That is, any $G$-invariant morphism $f: X \rightarrow Z$ uniquely factors over $q$.

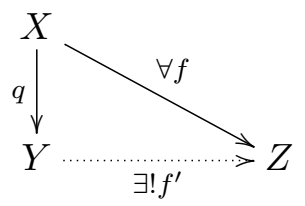

In particular, if a good quotient exists, it is unique up to unique isomorphism.

Moreover, one can show that two orbits $\mathcal{O}$ and $\mathcal{O}^{\prime}$ are mapped to the same point if and only if

$$
\overline{\mathcal{O}} \cap \overline{\mathcal{O}^{\prime}} \neq \emptyset
$$


Finally, we remark that a geometric quotient $q: X \rightarrow Y$ induces a geometric quotient

$$
q: U \rightarrow q(U)
$$

on any open and $G$-invariant subset $U \subset X$, and that $q(U) \subset Y$ is open. In general, this is not true for good quotients.

There is a very simple example illustrating these concepts.

EXAMPle 1.1.2. Let the torus $T=\mathbb{G}_{m}$ of rank one act on the variety $X=\mathbb{A}^{n}$ via scaling. A good quotient of this action exists, though it is the trivial morphism

$$
q: \mathbb{A}^{n} \rightarrow \operatorname{Spec}(k)
$$

because the origin is the unique closed orbit.

Note that if we remove the origin, all pointed lines become closed orbits, and indeed the well-known map

$$
q^{\prime}: \mathbb{A}^{n} \backslash 0 \rightarrow \mathbb{P}^{n-1}
$$

is a good and geometric quotient.

The general philosophy of removing a set of bad orbits is also found in the construction of quotients via Geometric Invariant Theory.

\subsubsection{GIT quotients.}

Let a reductive group $G$ act on a quasiprojective variety $X$, which we sometimes call the prequotient.

Mumford's Geometric Invariant Theory (GIT) is a powerful tool to find open subsets such that good quotients exist.

A $G$-line bundle $E \rightarrow X$ on $X$ is a line bundle $E \rightarrow X$, equipped with an action $G \times E \rightarrow E$ such that the diagram

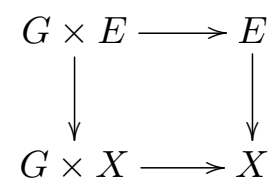

commutes, and the induced action on fibres of $E$ is linear. We refer to [KKLV 89] for more background on this notion.

Two $G$-line bundles $E, E^{\prime}$ are isomorphic if there exists an equivariant isomorphism of line bundles $E \rightarrow E^{\prime}$ (leaving the base space fixed).

Definition 1.1.3. $\mathrm{By} \mathrm{Pic}^{G}(X)$ we denote the group of isomorphism classes of $G$-line bundles on $X$. 
Interpreted correctly, $\operatorname{Pic}^{*}(*)$ is functorial.

Lemma 1.1.4. Consider the category $C$, where objects are pairs $(X, G)$ of algebraic groups acting on varieties $X$, and morphisms

$$
(f, \varphi):(X, G) \rightarrow(Y, H)
$$

are pairs of morphisms of varieties and algebraic groups respectively, such that $f(g * x)=\varphi(g) * f(x)$ for all $x \in X$ and $g \in G$. Then there is a contravariant functor

$$
\operatorname{Pic}^{*}(*): C \rightarrow A b,
$$

extending the usual functor $\operatorname{Pic}(*)$. If $(f, \varphi)$ is a morphism in $C$ as above, and $\chi \in \chi(H)$, then $(f, \varphi)^{*}\left(L_{\chi}\right)=L_{\varphi^{*}(\chi)}$.

Proof. We use the notation $(f, \varphi)$ for a morphism in $C$ as in the statement of the lemma. Recall that the pullback of an ordinary line bundle $\pi: E \rightarrow Y$ is given as

$$
f^{*}(E)=\{(x, e) \in X \times E \mid f(x)=\pi(e)\},
$$

and if additionally $E$ is an $H$-line bundle, we define $g *(x, e)=(g * x, \varphi(g) * e)$. Now, it is straightforward to check that this construction is well-defined and satisfies the assertions.

For a $G$-line bundle $E$ we denote by $H^{0}(X, E)^{G}$ the space of invariant sections, and by $R(X, E)^{G}$ the section ring consisting of invariant sections. We now want to associate the stable and semistable locus to a $G$-line bundle $E$. To avoid further complication, we assume that $E$ is ample as an ordinary line bundle.

Definition 1.1.5. To a $G$-line bundle $E$, which is ample as a line bundle, we associate the following loci.

(1) The semistable locus with respect to $E$ is defined as

$$
X^{E-\mathrm{sst}}=\bigcup_{f} \mathrm{D}(f),
$$

where $\mathrm{D}(f)=X \backslash \mathrm{N}(f)$, and the union is taken over all invariant sections $f \in H^{0}(X, m E)^{G}$, where $m \geq 1$.

(2) The stable locus $X^{E-\text { st }}$ is the set of points $x \in X^{E-\text { sst }}$ such that the stabilizer $G_{x}$ is finite, and the orbit $G * x$ is closed in the semistable locus.

(3) The unstable locus with respect to $E$ is the complement

$$
X \backslash X^{E-\text { sst }} .
$$

As is apparent from the definition,

$$
X^{E-\text { st }} \subset X^{E-\text { sst }} \subset X
$$

are open and $G$-invariant subsets, though they could possibly be empty. The technical cornerstone of GIT is the fact that, unless they are empty, quotients for these subsets exist. 
TheOREM 1.1.6. The evaluation of sections defines a good quotient

$$
q_{E}: X^{E-\text { sst }} \rightarrow Y_{E}=\operatorname{Proj}\left(R(X, E)^{G}\right),
$$

which restricts to a geometric quotient on the stable locus.

REMARK 1.1.7. By construction of the quotient $Y=Y_{E}$, the canonical line bundle $\mathcal{O}_{Y}(1)$ pulls back to $E$, i.e.

$$
q_{E}^{*}\left(\mathcal{O}_{Y}(1)\right)=E,
$$

where we use the notion of pullback as outlined in Lemma 1.1.4. If the quotient is projective, this could also be phrased as the statement that $E$ descends to an ample line bundle on the quotient.

A special case important to us is that of the linearization of the trivial line bundle on an affine variety.

Convention 1.1.8. We will denote the trivial line bundle by

$$
L \rightarrow X \text {. }
$$

As [KKV 89] shows, linearizations of the trivial line bundle are essentially given by a character

$$
\chi \in \chi(G)=\operatorname{Hom}\left(G, \mathbb{G}_{m}\right) .
$$

For such a character, $L_{\chi}$ is the corresponding $G$-line bundle with action

$$
g *(x, e)=(g * x, \chi(g) \cdot e) .
$$

A section $f \in H^{0}\left(X, L_{\chi}\right)^{G}$ is also called a semiinvariant and satisfies

$$
g * f=\chi(g) \cdot f .
$$

Note that some authors call a function as given above a semiinvariant of rank 1 . We do not need this distinction.

Convention 1.1.9. We abbreviate the semistable locus as

$$
X^{\chi-\text { sst }}=X^{L_{\chi}-\text { sst }}
$$

and similarly for the stable locus. We write the associated good quotient as

$$
q_{\chi}: X^{\chi-\text { sst }} \rightarrow Y_{\chi}
$$

\subsubsection{Variational GIT.}

The space $\operatorname{Pic}^{G}(X)_{\mathbb{R}}$ can be thought of as a parameter space for GIT, and it is interesting to ask, how the GIT quotient changes as we vary the choice of $E \in \mathrm{Pic}^{G}(X)$. The answer to this question is the theory of Variational Geometric Invariant Theory (VGIT). For general reductive groups, this was developed by Thaddeus (cf. [Th96]) and Dolgachev and Hu (cf. [DH98]). For the most part, they work over a normal and projective variety $X$. We also need VGIT on affine varieties, which was discussed by Halic (consider [Hal04]), at least for linearizations of the trivial line bundle. 
The main results of VGIT are about the $G$-ample cone

$$
C^{G}(X) \subset \mathrm{NS}^{G}(X)_{\mathbb{R}},
$$

which we now need to describe. The right hand side is a finite-dimensional replacement for the possibly infinite-dimensional space $\operatorname{Pic}^{G}(X)_{\mathbb{R}}$.

REMARK 1.1.10. In the convention of Thaddeus, the space $\mathrm{NS}^{G}(X)$ is given as the set of $G$-line bundles modulo equivariant algebraic equivalence (see [Th96], Section 2 for details). In the language of Dolgachev and $\mathrm{Hu}$, it is given by modding out homologically trivial line bundles which are trivially linearized (consider [DH98], Definition 2.3.4.).

In the cases we are interested in, we only want to vary linearizations of the trivial line bundle $L$, so that the subtle difference between $\operatorname{Pic}^{G}(X)$ and the two versions of $\mathrm{NS}^{G}(X)$ is of no further relevance.

Definition 1.1.11. The $G$-ample cone is the convex cone

$$
C^{G}(X) \subset \mathrm{NS}^{G}(X)_{\mathbb{R}}
$$

spanned by classes of $G$-line bundles $E$ which are ample as line bundles, and which are $G$-effective. That is,

$$
H^{0}(X, m E)^{G} \neq 0
$$

for some multiple.

For ample line bundles, the second condition is equivalent to

$$
X^{E-\text { sst }} \neq \emptyset \text {. }
$$

Definition 1.1.12. We say that two $G$-line bundles $E$ and $E^{\prime}$ are GIT equivalent if

$$
X^{E-\mathrm{sst}}=X^{E^{\prime}-\mathrm{sst}} .
$$

This also implies that the stable loci, as well as the associated quotients, on the stable and semistable locus, coincide.

The first main result of VGIT (consider [Th96], Theorems 2.3 and 2.4, as well as [DH98], Theorem 3.3.3 and Theorem 3.4.2) can now be phrased as follows.

TheOREM 1.1.13. Let $X$ denote a normal and projective variety.

The $G$-ample cone $C^{G}(X)$ is rational polyhedral. There are finitely many rational hyperplanes, called GIT walls, such that $E$ and $E^{\prime}$ are GIT equivalent if they can be connected by a path which does not cross a wall.

Here, we say that a path $\gamma$ crosses a wall $H$ if there exists a point $\gamma(a) \in H$ and a point $\gamma(b) \notin H$. In particular, a path can potentially start and end in some wall $H$ without crossing it.

Clearly, the walls divide the $G$-ample cone into finitely many pieces, which we call GIT chambers and cells.

Definition 1.1.14. Consider the wall structure on the ample cone $C^{G}(X)$ as discussed in Theorem 1.1.13.

A GIT chamber is a connected component of $C^{G}(X)$ with the walls removed. A GIT cell is a connected component of some wall $W$ without the intersections with any other, properly intersecting, wall. 
By Theorem 1.1.13 it is immediately clear that the GIT chambers are precisely the full-dimensional GIT equivalence classes, and that each GIT cell is contained in a single GIT equivalence class.

REMARK 1.1.15. There are some differing conventions for the notion of walls and chambers. In the language of [DH98], for $E$ inside a chamber we necessarily have

$$
X^{E-\mathrm{sst}}=X^{E-\mathrm{st}},
$$

although it is not explicitly required in the definition (consider [DH98], Theorem 3.3.2). On the other hand, their definition of a wall allows codimension zero walls.

Even worse, the definition of a chamber as used in Section 3.6.2, which discusses walls and chambers in the space of stability conditions for quiver sheaves, includes subsets which are not of full dimension. Additionally, the walls used there are not necessarily hyperplanes. Since both conventions seem to be standard in their respective field of study, we will use both in the according sections.

The second main result of VGIT states that the GIT quotients undergo birational transformations as the choice of the stability condition $E$ varies (compare with [Th96], Lemma 3.2 and Theorem 3.3, and [DH98], Lemma 4.2.1 and the discussion below that Lemma).

Suppose that $C_{+}$and $C_{-}$are chambers in $C^{G}(X)$, separated by a wall $H$. Assume that we may choose $G$-line bundles $E_{+} \in C_{+}$and $E_{-} \in C_{-}$which are linearization of the same (class of a) line bundle $E$. Then the line segment between them intersects the wall in a rational point, which is thus another linearization $E_{0} \in H$ of the line bundle $E$.

THEOREM 1.1.16. There are inclusions

$$
X^{E_{+}-\text {sst }} \subset X^{E_{0}-\text { sst }} \supset X^{E_{-}-\text {sst }},
$$

and a diagram

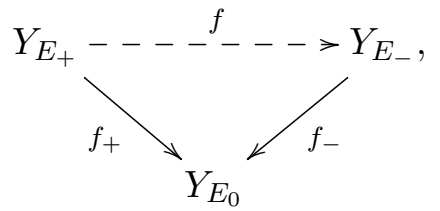

where $f_{+}$and $f_{-}$are proper and birational.

Actually, more is true. It is possible to construct a rational map

$$
f: Y_{E} \rightarrow Y_{E^{\prime}}
$$

from any $G$-line bundle $E$ in the interior of the $G$-ample cone to any other $G$-line bundle $E^{\prime} \in C^{G}(X)$. If $E^{\prime}$ is also contained in the interior, this map is birational. We will give a more explicit construction of this map below, at least for the case of linearizations of the trivial line bundle. 
REMARK 1.1.17. The rational map which is constructed in Theorem 1.1.16 is called a Thaddeus-flip, even though it is not necessarily a flip in the sense usually considered in birational geometry. Moreover, the same naming is used in other contexts of variation problems (eg. in [MW97] or [GRT16]). However, if the complements of the stable loci with respect to $E_{+}$and $E_{-}$ both have codimension at least 2 , the birational map $f$ is a flip, in the sense of birational geometry, with respect to the ample line bundle $\mathcal{O}_{Y_{+}}(1)$ as explained in Remark 1.1.7.

Now, we want to discuss the case of an affine and normal variety $X$, following [Hal04]. We can use the standard linearization

$$
X \hookrightarrow \mathbb{A}^{N}
$$

into a $G$-module $\mathbb{A}^{N}$, to give the description

$$
X^{\chi-\text { sst }}=X \cap\left(\mathbb{A}^{N}\right)^{\chi-\text { sst }},
$$

where $\chi \in \chi(G)$ is some character, and a similar description for the stable loci. Thus, at least for linearizations of the trivial line bundle, we have the same chamber behavior for the action of $G$ on $X$ as outlined for projective varieties above.

From now on, we want the following to hold.

Assumption 1.1.18. For all characters $\chi$ which are contained in a GIT chamber $C \subset \chi(G)_{\mathbb{R}}$ we have

$$
X^{\chi-\mathrm{st}}=X^{\chi-\mathrm{sst}} .
$$

Note that this may fail for all GIT chambers at once, or it may fail for some chambers, while it holds for others (consider the counterexample [Res98]). However, there are two important situations where our assumption is satisfied.

(1) This holds for the action of $P G_{d}$ on $R_{d}(Q)$, where $Q$ is a quiver (for the relevant definitions, see Section 1.2), and $d$ is a coprime dimension vector, i.e. the entries of $d$ admit no nontrivial common divisor (compare with Section 3.5 in [Rei08]).

(2) If $G=T$ is a torus, the fact that stability and semistability coincide for one chamber implies the same assertion for the other chambers (again we use a linearization to reduce to the case of a $T$-module, where it holds by Proposition 3.10 in [Hal04]).

Furthermore, we can use the linearization to transfer the Hilbert-Mumford criterion for $G$-modules, as established in [Hal04] or [K94], to an arbitrary normal affine variety. 
Next, we want to give a more explicit description for the rational maps

$$
f: Y_{\chi} \rightarrow Y_{\chi^{\prime}}
$$

between the quotients. Suppose that we are given two characters $\chi, \chi^{\prime}$ of $G$, and assume that $X^{\chi-s t}$ is nonempty. Setting

$$
V=X^{\chi-\mathrm{st}} \cap X^{\chi^{\prime}-\mathrm{sst}},
$$

the restriction $V \rightarrow q_{\chi}(V) \subset Y_{\chi}$ is again a geometric quotient, with open image. Further, the composition

$$
V \subset X^{\chi^{\prime}-\text { sst }} \rightarrow Y_{\chi^{\prime}}
$$

is $G$-invariant, and hence factors to give a morphism $q_{\chi}(V) \rightarrow Y_{\chi^{\prime}}$. This defines a rational map $f: Y_{\chi} \rightarrow Y_{\chi^{\prime}}$.

The situation is summarized in the following diagram.

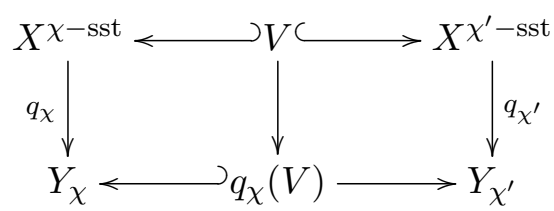

Note that $f$ is a birational map if $X^{\chi^{\prime}-\text { st }} \neq \emptyset$. Indeed, the images under $q_{\chi}$ and $q_{\chi^{\prime}}$ of

$$
V^{\prime}=X^{\chi-s t} \cap X^{\chi^{\prime}-\mathrm{st}}
$$

give open subsets $q_{\chi}\left(V^{\prime}\right) \subset Y_{\chi}$ and $q_{\chi^{\prime}}\left(V^{\prime}\right) \subset Y_{\chi^{\prime}}$, which are both geometric quotients of $V^{\prime}$, and are hence isomorphic.

\subsection{Quivers and their representations}

Quiver moduli were introduced to study the isomorphism classes of modules over artinian algebras (see [K94]). In this section, we recall the basic notions of a quiver and its representations. We also recall the concept of stability and the construction of a moduli space as introduced by King. For a concise introduction we refer the reader to [Rei08].

Furthermore, we develop an extension of these concepts to the case of labeled quivers, i.e. ordinary quivers with labeling vector spaces assigned to the arrows. This is a mostly straightforward procedure, but even though special cases have been studied (eg. in [ACK07] or [GRT16]), there seems to be no such concise treatment in the literature.

Definition 1.2.1. An (unlabeled) quiver $Q=\left(Q_{0}, Q_{1}\right)$ consists of a set of vertices $Q_{0}$, a set of arrows $Q_{1}$, and two functions

$$
t, h: Q_{1} \rightarrow Q_{0},
$$

assigning to an arrow $\alpha$ its tail and head.

Typically, arrows are denoted by $\alpha: i \rightarrow j$, where $i=t(\alpha)$ and $j=h(\alpha)$. We further assume that both sets $Q_{0}, Q_{1}$ are finite. 
Suppose that there are precisely $n$ distinct arrows

$$
1 \stackrel{n}{\longrightarrow} 2
$$

from vertex 1 to vertex 2 . Often, we are not interested in the concrete set of arrows, but just their joint properties, i.e. the sum of their images. This gives rise to the idea to replace them with a vector space $H$ of dimension $n$.

Definition 1.2.2. A labeling for a quiver $Q$ is a collection of vector spaces

$$
H=\left(H_{\alpha} \mid \alpha \in Q_{1}\right)
$$

of finite dimension for each arrow in $Q$. An arrow $\alpha$ with label $H_{\alpha}=k$ is considered to be unlabeled. The pair $(Q, H)$ is called a labeled quiver.

We think of an unlabeled quiver as the special case, where the labeling is trivial, i.e. $H_{\alpha}=k$ for all arrows.

\subsubsection{The category of representations.}

Usually, one is not very interested in the quiver itself, but in its representations, which are a very well-established concept.

A representation $M$ of an (unlabeled) quiver $Q$ in some category $\mathcal{C}$ consists of a tuple of objects $\left(M_{i} \mid i \in Q_{0}\right)$ for each vertex, and a tuple of morphisms

$$
\left(M_{\alpha}: M_{i} \rightarrow M_{j} \mid(\alpha: i \rightarrow j) \in Q_{1}\right)
$$

for each arrow. Together with the appropriate notion of morphisms, these representations form a category.

Definition 1.2.3. The category of representations of $Q$ in the category $C$ is denoted as $Q-\operatorname{rep}_{\mathcal{C}}$.

The special cases of representations in the category $k$ - vect of vector spaces of finite dimension, and in the category of coherent sheaves $\operatorname{Coh}(X)$ on some scheme $X$ over $k$ deserve the special notations

$$
Q-\operatorname{rep}=Q-\operatorname{rep}_{k-\text { vect }}, \quad Q-\operatorname{Coh}(X)=Q-\operatorname{rep}_{\operatorname{Coh}(X)} .
$$

In Chapter 3, we further discuss objects in $Q-\operatorname{Coh}(X)$, which are called quiver sheaves. 
A representation of a labeled quiver is given in a slightly different manner.

Definition 1.2.4. A representation $M$ of a labeled quiver $(Q, H)$ consists of a tuple $\left(M_{i} \mid i \in Q_{0}\right)$ of finite-dimensional vector spaces, and a tuple

$$
\left(M_{\alpha}: M_{i} \otimes_{k} H_{\alpha} \rightarrow M_{j} \mid(\alpha: i \rightarrow j) \in Q_{1}\right)
$$

of linear maps. A morphism of representations $\varphi: M \rightarrow N$ consists of a tuple of linear maps $\left(\varphi_{i}: M_{i} \rightarrow N_{i} \mid i \in Q_{0}\right)$, such that for all arrows $\alpha: i \rightarrow j$ in $Q$ the diagram

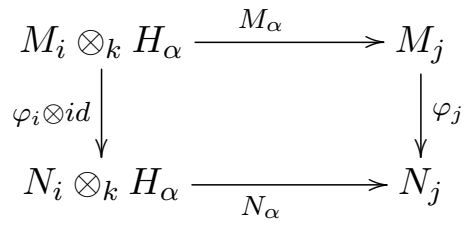

commutes.

Clearly, we recover the unlabeled case if we delete all occurrences of $\otimes_{k} H_{\alpha}$. Equivalently we can use the canonical identification $V \otimes_{k} k \simeq V$. Again, the representations form a category.

Definition 1.2.5. The category of representations of $(Q, H)$ is denoted as $(Q, H)$ - rep.

We also need the dimension vector of a representation.

Definition 1.2.6. The dimension vector of a vector space representation $M$ is defined as

both for the labeled and unlabeled case.

$$
\underline{d}(M)=\left(\operatorname{dim}\left(M_{i}\right) \mid i \in Q_{0}\right),
$$

In the unlabeled case, relations on the quiver are a well-established concept. To the best knowledge of the authors, there is no previous treatment of relations in the case of a labeled quiver.

We need such relations. More precisely, we need one very special case involving non-trivial labels, and relations only involving unlabeled arrows. Developing a more general theory seems tedious and technical, with no clear application.

Convention 1.2.7. Suppose that $(Q, H)$ contains a subquiver

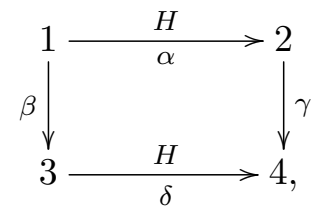

that is two unlabeled arrows $\beta, \gamma$ and two labeled arrows $\alpha, \delta$, with the same label $H$ on opposite sides of a square. We then say that a representation $M$ satisfies the relation $\gamma \alpha-\delta \beta$ if there is a commuting diagram

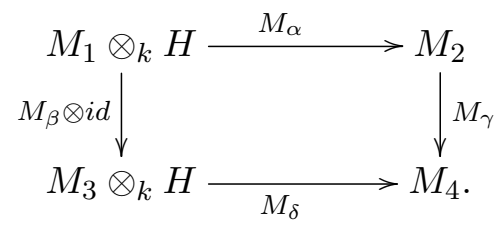


Again, after removing the terms $\otimes_{k} H$, we arrive at a very special case of a relation in the unlabeled case.

Another special case we need is that of relations which only involve unlabeled arrows. We recall that a path is simply a sequence

$$
\gamma=\alpha_{l} \ldots \alpha_{1}
$$

of arrows such that the tail of each arrow is the head of its predecessor. The relations we are interested in are then of the form

$$
\sum_{k=1}^{r} \lambda_{k} \gamma_{k}
$$

where the $\lambda_{k}$ are scalars, and all the $\gamma_{k}: i \rightsquigarrow j$ are non-trivial paths starting in the same vertex $i$ and ending in the same vertex $j$, such that the arrows which compose $\gamma$ are unlabeled.

For a path $\gamma=\alpha_{l} \ldots \alpha_{1}: i \rightsquigarrow j$ composed of unlabeled arrows, and a representation $M$ we denote

$$
M_{\gamma}=M_{\alpha_{l}} \ldots M_{\alpha_{1}}: M_{i} \rightarrow j,
$$

and say that $M$ satisfies a relation as given above if

$$
\sum_{k=1}^{r} \lambda_{k} M_{\gamma}=0 .
$$

Later in the discussion, we will provide a more general discussion of paths, allowing non-trivial labels.

We further remark that relations in the unlabeled case also make sense for arbitrary $k$-linear categories. In particular, we can consider quiver sheaves satisfying a set of relations $I$.

Definition 1.2.8. The full subcategory of quiver sheaves satisfying the relations $I$ is denoted as

$$
(Q, I)-\operatorname{Coh}(X) \subset Q-\operatorname{Coh}(X) .
$$

A labeled quiver with relations is now defined as a triple $(Q, H, I)$, where $(Q, H)$ is a labeled quiver and $I$ is a set of relations of a form as discussed above.

Definition 1.2.9. The category

$$
(Q, H, I)-\operatorname{rep} \subset(Q, H) \text { - rep }
$$

is the full subcategory of representations which satisfy the relations $I$.

The connection of the notion of labeled representations to the unlabeled ones is via a choice of basis for each label $H_{\alpha}$. This also allows us to inherit many already established results.

Specifically, let $(Q, H, I)$ denote any labeled quiver with relations. Construct a new (unlabeled) quiver $Q^{\prime}$ by setting

$$
Q_{0}^{\prime}=Q_{0}, Q_{1}^{\prime}=\left\{\alpha_{k}: i \rightarrow j \mid(\alpha: i \rightarrow j) \in Q_{1}, k=1, \ldots, \operatorname{dim}\left(H_{\alpha}\right)\right\} .
$$


Roughly speaking, we replace each arrow by $\operatorname{dim}\left(H_{\alpha}\right)$ copies. For a relation $\gamma \alpha-\delta \beta$ in a form as explained above, we equip $Q^{\prime}$ with $\operatorname{dim}(H)$ relations of the form

$$
\gamma \alpha_{k}-\delta_{k} \beta, \quad k=1, \ldots, \operatorname{dim}(H) .
$$

Relations only involving unlabeled arrows can be imposed on $Q^{\prime}$ in a straightforward way. The set of all such relations is denoted as $I^{\prime}$.

If $\theta$ is a stability condition on $Q$, as discussed in Subsection 1.2.2, it is also possible to consider it as a stability condition on $Q^{\prime}$.

Now, choose a basis for each label $H_{\alpha}$. We restrict this choice by assuming the following.

(1) If the label is trivial, i.e. $H_{\alpha}=k$, the canonical basis $1 \in k$ is chosen.

(2) If two labels are exactly the same, i.e. $H_{\alpha}=H_{\beta}$, the same bases are chosen.

Proposition 1.2.10. The choice of bases induces an isomorphism of categories

$$
(Q, H, I)-\text { rep } \rightarrow\left(Q^{\prime}, I^{\prime}\right)-\text { rep. }
$$

This identification respects dimension vectors.

Proof. We sketch the definition of the involved functors. Extending them to morphisms, and checking the identities is then straightforward.

The choice of bases $\mathrm{B}_{\alpha}=\left(h_{1}, \ldots, h_{n_{\alpha}}\right)$, where $n_{\alpha}=\operatorname{dim}\left(H_{\alpha}\right)$, induces direct sum decompositions

$$
M_{i} \otimes H_{\alpha}=\bigoplus_{l=1}^{n_{\alpha}} M_{i} \otimes_{k} k \cdot h_{l} \simeq \bigoplus_{l=1}^{n_{\alpha}} M_{i} .
$$

The associated projections and inclusions (composed with identification) are denoted as $\pi_{l}$ and $\varepsilon_{l}$ respectively. Suppose we are given a representation $M$ of $(Q, H, I)$. Define a representation $M^{\prime}$ by

$$
M_{i}^{\prime}=M_{i} \text { for all } i \in Q_{0} \text { and } M_{\alpha_{l}}^{\prime}=M_{\alpha} \varepsilon_{l} \text { for the arrows. }
$$

Our restrictions on the choices of bases imply, that the representation $M^{\prime}$ satisfies the relations $I^{\prime}$. Conversely, suppose that $M^{\prime}$ is a given representation of $\left(Q^{\prime}, I^{\prime}\right)$. Then $M$ can be constructed via

$$
M_{i}=M_{i}^{\prime} \text { for all } i \in Q_{0} \text {, and } M_{\alpha}=\sum_{l=1}^{n_{\alpha}} M_{\alpha_{l}}^{\prime} \pi_{l} \text { for the arrows. }
$$

It is obvious that dimension vectors are respected.

The paths in a labeled quiver $(Q, H)$ are simply paths in the underlying quiver $Q$, and the label of a path $\gamma=\alpha_{1} \alpha_{2} \ldots \alpha_{l}$ is defined as

$$
H_{\gamma}=H_{\alpha_{l}} \otimes_{k} \ldots \otimes_{k} H_{\alpha_{1}} \text {. }
$$

By convention, the paths $e_{i}$ of length zero should be labeled by $k$. 
Definition 1.2.11. The path algebra of $(Q, H)$ is

$$
A=A(Q, H)=\bigoplus_{\gamma \text { path }} k \cdot \gamma \otimes_{k} H_{\gamma}
$$

as a vector space, and multiplication is given on homogeneous elements by

$$
(\gamma \otimes h) \cdot\left(\gamma^{\prime} \otimes h^{\prime}\right)=\gamma \gamma^{\prime} \otimes\left(h^{\prime} \otimes h\right)
$$

if the concatenation $\gamma \gamma^{\prime}$ is possible, and zero otherwise. Relations of the form $\delta \beta-\gamma \alpha$ in $(Q, H)$ give rise to the relations

$$
\left(\delta \otimes h_{\delta}\right) \cdot\left(\beta \otimes h_{\beta}\right)-\left(\gamma \otimes h_{\gamma}\right) \cdot\left(\alpha \otimes h_{\alpha}\right)=0
$$

independent of the elements $h_{\alpha}, h_{\delta} \in H$ and $h_{\beta}, h_{\gamma} \in k$. The path algebra with relations is then simply the path algebra of $(Q, H)$ modulo the ideal generated by these relations.

Again, removing $\otimes_{k} H_{\alpha}$ from the definition recovers the path algebra of an unlabeled quiver. Similarly to the argument above, we can show that there is an identification of the labeled and unlabeled case.

Proposition 1.2.12. There is an isomorphism

$$
A \simeq A^{\prime}
$$

between the path algebra $A$ of $(Q, H, I)$ and the path algebra $A^{\prime}$ of $\left(Q^{\prime}, I^{\prime}\right)$.

As a first example of the translation of results from the unlabeled to the labeled case, we recall and proof the identification of modules over the path algebra and representations. Let $A$ denote the path algebra of $(Q, H, I)$ and let $A^{\prime}$ denote the path algebra of the associated unlabeled quiver $\left(Q^{\prime}, I^{\prime}\right)$.

Proposition 1.2.13. There is a commuting diagram of equivalences of categories

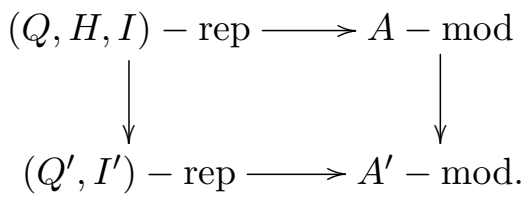

Proof. The vertical arrows are given by Proposition 1.2.12 and Proposition 1.2.10. The functor in the upper row is given as follows.

For a representation $M$ of $(Q, H, I)$, the corresponding $A$-module is given as

$$
M=\bigoplus_{i \in Q_{0}} M_{i},
$$

where an element $\alpha \otimes h \in A$ for an arrow $\alpha: i \rightarrow j$ acts as

$$
(\alpha \otimes h) \cdot m=M_{\alpha}(m \otimes h)
$$

from the $i$-th to the $j$-th summand, and as zero otherwise. Conversely, suppose we are given an $A$-module $M$. Since the elements $e_{i} \otimes 1$ for $i \in Q_{0}$ form a decomposition of unity into indecomposable orthogonal idempotents, there is a decomposition

$$
M=\bigoplus_{i \in Q_{0}} M_{i}=\bigoplus_{i \in Q_{0}}\left(e_{i} \otimes 1\right) M,
$$


which we take as the collection of vector spaces for a representation. An arrow $\alpha: i \rightarrow j$ is equipped with the map

$$
\left(e_{i} \otimes 1\right) M \otimes_{k} H_{\alpha} \rightarrow\left(e_{j} \otimes 1\right) M,\left(e_{i} \otimes 1\right) m \otimes h \mapsto(m \otimes h)\left(e_{i} \otimes 1\right) m .
$$

This construction respects choices of bases, and dropping the terms $\otimes_{k} H_{\alpha}$ gives a similar functor for the lower row, making the diagram commute. The lower row given by this is well-known to be an equivalence.

\subsubsection{Stability conditions.}

To construct meaningful moduli spaces, we need to restrict to semistable representations as introduced by King. His original work was concerned with the unlabeled case, but there is no difficulty in extending the definitions to the labeled case.

A stability condition on a quiver, labeled or unlabeled, is a tuple $\theta \in \mathbb{R}^{Q_{0}}$.

Definition 1.2.14. The slope of a representation with respect to $\theta$ is given as

$$
\mu(M)=\frac{\sum_{i \in Q_{0}} \theta_{i} \operatorname{dim}\left(M_{i}\right)}{\sum_{i \in Q_{0}} \operatorname{dim}\left(M_{i}\right)},
$$

and $M$ is said to be $\theta$-semistable if and only if

$$
\mu(N) \leq \mu(M)
$$

holds for all non-trivial subrepresentations $N \subset M$. If strict inequality holds for all subrepresentations we say that $M$ is stable. Consequently, for some representation $M$ we say that $N \subset M$ is destabilizing if $\mu(N) \geq \mu(M)$.

The full subcategory of semistable representations of fixed slope is closed under kernels, cokernels and extensions, and hence abelian. The stable ones are exactly the simple objects in this category, and objects are artinian. This gives us the following variant of the Jordan-Hölder filtration.

Proposition 1.2.15. Let $M$ denote a semistable representation of some quiver (labeled or unlabeled). Then there exists a filtration

$$
0=M^{0} \subsetneq M^{1} \subsetneq M^{2} \subsetneq \ldots \subsetneq M^{l}=M
$$

of semistable subrepresentations of the same slope, such that the subquotients $M^{k+1} / M^{k}$ are stable. Moreover, the subquotients are uniquely determined in any such filtration up to isomorphism and permutation.

The uniqueness of the subquotients allows us to define $S$-equivalence of representations.

Definition 1.2.16. For a semistable representation $M$ we define the graded representation as

$$
\operatorname{gr}(M)=\bigoplus_{k=1}^{l} M^{k} / M^{k-1},
$$

where the $M^{k}$ comprise some Jordan-Hölder filtration of $M$. We say that two semistable representations $M$ and $M^{\prime}$ are $S$-equivalent if $\operatorname{gr}(M) \simeq$ $\operatorname{gr}\left(M^{\prime}\right)$. 
Consider the identification of Proposition 1.2.10. Since $Q$ and $Q^{\prime}$ have the same set of vertices, a stability condition $\theta$ can be used on both simultaneously. Subrepresentations and dimension vectors are preserved, so that stability is preserved as well.

COROLlary 1.2.17. The identification

$$
(Q, H, I)-\operatorname{rep} \simeq\left(Q^{\prime}, I^{\prime}\right)-\text { rep }
$$

preserves stability, semistability and $S$-equivalence.

\subsubsection{Moduli spaces.}

The method of construction of a moduli space of semistable quiver representations is to parametrize representations by some variety $R_{d}(Q, H, I)$, with a canonical group action which encodes isomorphisms. In the next step, we relate stability of representations with stability on the variety in the sense of GIT, and the associated quotient is the moduli space. This program was first carried out by [K94].

Let $(Q, H, I)$ denote a labeled quiver with relations.

Definition 1.2.18. The representation variety for dimension vector $d \in \mathbb{N}^{Q_{0}}$ is given as

$$
R_{d}(Q, H)=\bigoplus_{\alpha: i \rightarrow j} \operatorname{Hom}\left(k^{d_{i}} \otimes H_{\alpha}, k^{d_{j}}\right),
$$

and the relations $I$ define a closed subvariety $R_{d}(Q, H, I) \subset R_{d}(Q, H)$.

On $R_{d}(Q, H)$ we have an action of the group

$$
G_{d}=\prod_{i \in Q_{0}} \mathrm{GL}\left(d_{i}, k\right)
$$

via conjugation, that is

$$
\left(g_{i} \mid i \in Q_{0}\right) *\left(f_{\alpha} \mid \alpha: i \rightarrow j\right)=\left(g_{j}^{-1} f_{\alpha}\left(g_{i} \otimes i d\right) \mid \alpha: i \rightarrow j\right) .
$$

Note that the diagonally embedded scalars $\mathbb{G}_{m} \subset G_{d}$ act trivially, so that we can equivalently pass to the action of the group

$$
P G_{d}=G_{d} / \mathbb{G}_{m} \text {. }
$$

Furthermore, there is a tautological bundle $\mathbb{M}$ of $A$-modules On $R_{d}(Q, H, I)$. Here, $A$ denotes the path algebra of $(Q, H, I)$.

As a vector bundle, $\mathbb{M}$ is trivial

$$
\mathbb{M}=\left(\bigoplus_{i \in Q_{0}} k^{d_{i}}\right) \times R_{d}(Q, H, I) \rightarrow R_{d}(Q, H, I) .
$$

Each fiber $\mathbb{M}_{x}$ carries the structure of an $A$-module, by declaring that each arrow acts via the morphisms encoded in $x$.

Clearly, any representation $N$ of $(Q, H, I)$ with dimension vector $d$ is isomorphic to a member of this family (identifying $N_{i} \simeq k^{d_{i}}$ ), and hence gives a point in $R_{d}(Q, H, I)$. Isomorphism classes of representations correspond 
to orbits under the action of $G_{d}$.

This construction is compatible with choices of bases, and restricts to the well-known case of unlabeled quivers. Hence, we can transfer the following theorem due to [K94]. Note, that we may assume $\theta \in \mathbb{Z}^{Q_{0}}$ without loss of generality (see Remark 1.2.22), and define a character via the following well-known identification.

Lemma 1.2.19. Mapping a tuple $\theta \in \mathbb{Z}^{Q_{0}}$ to the character

$$
\chi_{\theta}: G_{d} \rightarrow \mathbb{G}_{m}, \quad\left(g_{i} \mid i \in Q_{0}\right) \mapsto \prod_{i \in Q_{0}} \operatorname{det}\left(g_{i}\right)^{-\theta_{i}}
$$

induces an isomorphism $\mathbb{Z}^{Q_{0}} \simeq \chi\left(G_{d}\right)$. The characters of $P G_{d}$ correspond to tupels orthogonal to $d$.

On $R_{d}(Q, H, I)$, we hence have the notion of stability and semistability in the sense of GIT by considering the trivial line bundle $L$, linearized with $\chi_{\theta}$.

Proposition 1.2.20. Orbits in $R_{d}(Q, H, I)$ are in one-to-one correspondence with isomorphism classes of representations of $(Q, H, I)$ with dimension vector $d$. Stable and semistable representations correspond to stable and semistable orbits respectively, and two representations are $S$-equivalent if and only if the closures of the corresponding orbits intersect.

This implies that there is a GIT-construction of the moduli space. Here, we use the abbreviations st $=\theta-$ st and sst $=\theta-$ sst.

TheOREM 1.2.21. There is a commuting diagram

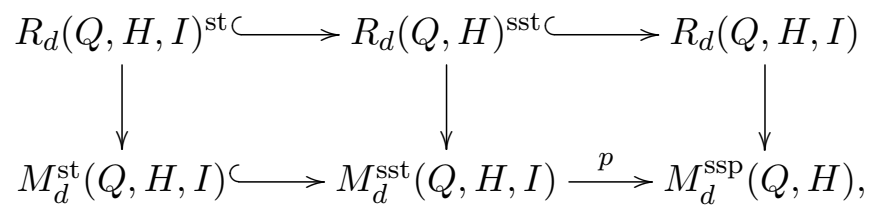

and the following assertions hold.

(1) The vertical arrows are good quotients, and the leftmost quotient is even geometric.

(2) The inclusions are open, and $p$ is a projective morphism.

(3) The varieties $M_{d}^{\mathrm{st}}(Q, H, I), M_{d}^{\mathrm{sst}}(Q, H, I)$ and $M_{d}^{\mathrm{ssp}}(Q, H, I)$ are the moduli spaces of stable, semistable and semisimple representations of $(Q, H, I)$ of dimension vector $d$, respectively.

(4) The variety $M_{d}^{\mathrm{ssp}}(Q, H, I)$ is affine. It is trivial if and only if $Q$ does not contain oriented cycles.

(5) Closed points in $M_{d}^{\text {sst }}(Q, H, I)$ correspond to $S$-equivalence classes of semistable representations, and closed points in $M_{d}^{\mathrm{st}}(Q, H, I)$ correspond to isomorphism classes of stable representations.

Proof. The special case of an unlabeled quiver without relations is settled by [K94], and the general theory of GIT (see eg. [Rei08], Section 3.5), and the question when $M_{d}^{\mathrm{ssp}}(Q)$ is affine is settled by [LP90].

Relations in the unlabeled case can be handled, once we note that $S_{-}$ equivalence respects relations. This is because orbit closure respects $S_{-}$ equivalence and

$$
R_{d}(Q, I)^{\mathrm{sst}}=R_{d}(Q, I) \cap R_{d}(Q)^{\mathrm{sst}} .
$$


Finally, our identifications allow a transfer to the labeled case. Note that $Q^{\prime}$ contains oriented cycles if and only if $Q$ does.

REMARK 1.2.22. As seen above, an integral tuple $\theta \in \mathbb{Z}^{Q_{0}}$ defines a character of $G_{d}$ such that stability with respect to the $G$-line bundle $L_{\chi}$ on $R_{d}(Q, H, I)$ is related to $\theta$-stability of representations. For arbitrary $\theta \in \mathbb{Q}^{Q_{0}}$ we can reduce to this case by scaling with a positive rational (and actually integral) number, allowing an extension of the definition of stability.

According to VGIT (see Section 1.1), the vector space $\mathbb{Q}^{Q_{0}}$ is divided into finitely many rational polyhedral chambers such that the behavior of stability does not depend on the explicit choice of $\theta$ inside any fixed chamber (this can also be seen by more concrete calculations, see eg. [Ch08]). The walls inducing this decomposition are the hyperplanes orthogonal to potential dimension vectors $e \leq d$ of subrepresentations, and hence rational. Using the closures of these walls, it is possible to extend the notion of stability to arbitrary real tuples $\theta \in \mathbb{R}^{Q_{0}}$.

Denote by $A$ the path algebra of $(Q, H, I)$, by $R$ the representation variety $R_{d}(Q, H, I)$, and by $G$ the group $G_{d}$. Consider the functor

$$
\mathcal{M}_{A}:(S c h / k)^{\mathrm{op}} \rightarrow \text { Sets },
$$

which maps a scheme $S$ to the set of isomorphism classes of $A \otimes \mathcal{O}_{S}$-modules which are locally free as $\mathcal{O}_{S}$-modules.

By sending a morphism $f: S \rightarrow R$ to the pullback $f^{*} \mathbb{M}$ of the tautological family, we obtain a natural transformation

$$
h: \underline{R} \rightarrow \mathcal{M}_{A} .
$$

Just like in [ACK07], Proposition 4.4, we can prove that $\mathcal{M}_{A}$ is locally isomorphic to a quotient functor.

Proposition 1.2.23. The natural transformation $h$ induces a local isomorphism

$$
h^{\prime}: \underline{R} / \underline{G} \rightarrow \mathcal{M}_{A} .
$$

\subsection{Rational maps and divisors}

In this section, we review some basic notions and conventions concerning divisors and rational maps.

The notion of a rational contraction is given as in the foundational paper [HK00]. We sometimes use equivalent descriptions provided by [Ca12]. For divisors, the standard reference is the book [Laz04] of Lazarsfeld.

\subsubsection{Divisors.}

We will often consider normal and projective varieties $X$ which are $\mathbb{Q}$ factorial. That is,

$$
\mathrm{Cl}(X)_{\mathbb{Q}}=\mathrm{CaCl}(X)_{\mathbb{Q}},
$$

i.e. every Weil divisor is $\mathbb{Q}$-Cartier. Recall that the Cartier Class group can be identified with the group $\operatorname{Pic}(X)$ of line bundles on $X$, where $\mathcal{O}(D)$ is 
the line bundle corresponding to a divisor $D$.

Let $D$ denote a Cartier divisor on a projective and normal variety $X$. The section ring of $D$ is defined as

$$
R(X, D)=\bigoplus_{m \geq 0} H^{0}(X, m D)=\bigoplus_{m \geq 0} H^{0}\left(X, \mathcal{O}_{X}(m D)\right) .
$$

Here, and elsewhere sometimes, we speak of sections of $\mathcal{O}(D)$ as sections of $D$ by a slight abuse of notation.

If $R(X, D)$ is finitely generated (as a $k$-algebra), then evaluation of sections gives rise to a rational map

$$
f_{D}: X \rightarrow Y_{D}=\operatorname{Proj}(R(X, D)),
$$

which is regular outside the stable base locus of $D$.

Here, the stable base locus is defined as the intersection

$$
B(D)=\bigcap_{m \geq 0} B s(m D)
$$

of the base loci of its multiples.

REMARK 1.3.1. Taking a thinning

$$
R(X, D)^{[k]}=\bigoplus_{m \geq 0} H^{0}(X, \mathcal{O}(k m D))
$$

does not change the projective spectrum, or the question whether the ring is finitely generated. Thus, the maps $f_{k D}$ are essentially the same for sufficiently large multiples $k$. Actually, they are Mori equivalent (consider Definition 1.3 .7 below).

Similarly to the situation in Remark 1.1.10, we need an appropriate quotient of the space $\operatorname{Pic}(X)$. That is, we consider the space

$$
N^{1}(X)=\mathrm{Ca}(X) / \equiv_{\text {num }}
$$

of Cartier divisors up to numerical equivalence. By definition, two divisors $D_{1}$ and $D_{2}$ are numerically equivalent if for all curves $C$ we have

$$
D_{1} \cdot C=D_{2} \cdot C \text {. }
$$

Recall that $N^{1}(X)_{\mathbb{R}}$ is a free abelian group of finite rank, and that this rank

$$
\rho(X)=\operatorname{rk}\left(N^{1}(X)\right)
$$

is called the Picard number of $X$. Inside $N^{1}(X)_{\mathbb{R}}$ one is often interested in the subcones

$$
\operatorname{Amp}(X) \subset \operatorname{Nef}(X) \subset \overline{\operatorname{Mov}}(X) \subset \overline{\operatorname{Eff}}(X) \subset N^{1}(X)_{\mathbb{R}},
$$

which we now want to describe.

It is well-known that a Cartier divisor $D$ is called ample if

$$
f_{k D}: X \hookrightarrow \operatorname{Proj}(R(X, k D))
$$


is a closed embedding for some multiple $k$, and that the classes of ample divisors span a cone

$$
\operatorname{Amp}(X) \subset N^{1}(X)_{\mathbb{R}}
$$

which is open. By the Kleiman-criterion, the closure of the ample cone is the nef cone, i.e.

$$
\operatorname{Nef}(X)=\overline{\operatorname{Amp}(X)} .
$$

By definition, it is spanned by classes of Cartier divisors $D$ such that

$$
D \cdot C \geq 0
$$

holds for all curves $C$. In between, there is the cone of semiample divisors

$$
\operatorname{Amp}(X) \subset \operatorname{SAmp}(X) \subset \operatorname{Nef}(X),
$$

which is spanned by classes of divisors $D$ such that some multiple is base point free. This cone is neither closed nor open in general.

Clearly, for a semiample divisor $D$, the associated map

$$
f_{D}: X \rightarrow Y_{D}
$$

is regular (if it exists). Even more, this map is a regular contraction, i.e. it is surjective and and has connected fibers.

However, the map

$$
f_{D}: X \rightarrow Y_{D}
$$

may be regular even though $D$ is not semiample. For an example, consider the Hirzebruch surface $\mathcal{F}_{1}$ (see Example 2.4.3).

A Cartier divisor $D$ is called movable if

$$
\operatorname{codim}(B(D)) \geq 2 .
$$

Clearly, semiample divisors are movable, so that there is an inclusion

$$
\operatorname{Nef}(X) \subset \overline{\operatorname{Mov}}(X) .
$$

Here, $\overline{\operatorname{Mov}}(X)$ is the closure of the cone which is spanned by classes of movable divisors.

Finally, we consider the cone of pseudoeffective divisors

$$
\overline{\operatorname{Eff}}(X) \subset N^{1}(X)_{\mathbb{R}} .
$$

It is the closure of the cone spanned by Cartier divisors $D$ which are effective, that is such that

$$
H^{0}(X, m D) \neq 0
$$

for some multiple $m$.

We remark that the interior of $\overline{\operatorname{Eff}}(X)$ is the so called big cone, spanned by classes of divisors $D$ such that

$$
f_{D}: X \rightarrow Y_{D}
$$

is a birational map. 


\subsubsection{Rational contractions.}

Let $f: X \rightarrow Y$ denote a dominant birational map of projective and normal varieties, where additionally $X$ is $\mathbb{Q}$-factorial.

Definition 1.3.2. We say that $f$ is a birational contraction if there exists a resolution

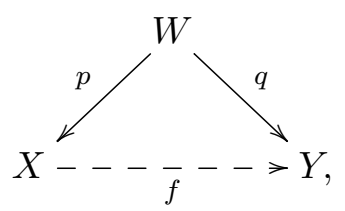

where $W$ is normal and projective, $p$ is birational, and for every $p$-exceptional effective divisor $E$ on $W$ the equation

$$
q_{*}\left(\mathcal{O}_{W}(E)\right) \simeq \mathcal{O}_{Y}
$$

holds. For a $\mathbb{Q}$-Cartier divisor $D$ on $Y$ the pullback is defined as

$$
f^{*}(D)=p_{*}\left(q^{*}(D)\right) .
$$

REMARK 1.3.3. All these notions do not depend on the choice of a resolution. The pullback is well-defined on numerical equivalence classes (cf. [Ca12], Lemma 1.3), but not functorial in general.

The rational map

$$
f_{m D}: X \rightarrow Y_{D},
$$

for a suitable multiple of a $\mathbb{Q}$-Cartier divisor $D$ with finitely generated section ring, provides a natural example of a rational contraction.

Definition 1.3.4. A small $\mathbb{Q}$-factorial modification (SQM) of a normal and projective variety $X$ is a birational map

$$
f: X \rightarrow Y
$$

to a normal, projective, and $\mathbb{Q}$-factorial variety which is an isomorphism in codimension one.

The map $f$ above is automatically a rational contraction. This is implied by the following characterization (consider [Ca12], Remark 2.2). This also shows that the inverse map $f^{-1}$ is a contraction as well.

Lemma 1.3.5. Consider a birational map $f: X \rightarrow Y$ between normal and projective varieties such that $X$ is additionally $\mathbb{Q}$-factorial. Then $f$ is a rational contraction if and only if there are open subsets $U \subset X$ and $X \subset Y$ such that

and $\operatorname{codim}(Y \backslash V) \geq 2$.

$$
f: U \stackrel{\sim}{\rightarrow} V
$$


REMARK 1.3.6. It is possible to define rational contractions

$$
f: X \rightarrow Y
$$

which are not necessarily birational, though the definition is rather technical ([HK00], Definition 1.1), and we make no use of it.

If $X$ is a Mori dream space it can be shown a posteriori, that all rational contractions factor as a SQM followed by a regular contraction (consider [Ca12], Proposition 3.3).

Definition 1.3.7. Let $D_{1}$ and $D_{2}$ denote $\mathbb{Q}$-Cartier divisors on a normal and projective variety $X$ such that their section rings are finitely generated. We say that $D_{1}$ and $D_{2}$ are Mori equivalent, if for some multiple $m>0$, the associated contractions are isomorphic. That is, there exists an isomorphism $\varphi$ between $Y_{D_{1}}=Y_{m D_{1}}$ and $Y_{D_{2}}=Y_{m D_{2}}$, such that the following diagram commutes.

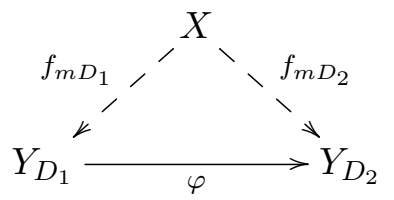

\subsection{Mori dream spaces}

Mori dream spaces were introduced by $\mathrm{Hu}$ and Keel in their foundational paper [HK00]. They form a class of varieties where Mori's program works very well, whence the name.

There are two different approaches to discuss the properties of a Mori dream space. One approach uses the cone structure inside the space of divisors, while it is also possible to describe it by VGIT of its Cox ring. In this section, we provide an overview of both points of view.

\subsubsection{Characterization via cones of divisors.}

The original definition of a Mori dream space ([HK00], Definition 1.10) characterizes it via its cone structure.

Definition 1.4.1. A projective and normal variety $Y$ is called a Mori dream space if the following holds.

(1) $Y$ is $\mathbb{Q}$-factorial, and $\operatorname{Pic}(Y)_{\mathbb{Q}}=N^{1}(Y)_{\mathbb{Q}}$.

(2) The nef cone $\operatorname{Nef}(Y)$ is spanned by finitely many semiample line bundles.

(3) There is a finite collection of SQMs

$$
f_{i}: Y \rightarrow Y_{i}, \quad i=1, \ldots, N
$$

such that each $Y_{i}$ satisfies (2), and the movable cone $\overline{\operatorname{Mov}}(Y)$ is the union of the cones $f_{i}^{*}\left(\operatorname{Nef}\left(Y_{i}\right)\right)$.

The second condition implies that the cones $\operatorname{Nef}\left(Y_{i}\right)$ are rationally polyhedral, so that $\overline{\operatorname{Mov}}(Y)$ is rational polyhedral as well.

If $f_{i}$ and $f_{j}$ are Mori equivalent, the pullbacks of the nef cones coincide, so 
that we can assume the $f_{i}$ to be pairwise inequivalent.

We state some of the good properties of a Mori dream space (consider [HK00], Proposition 1.11).

TheOrem 1.4.2. Let $Y$ denote a Mori dream space. Then the following holds.

(1) For all line bundles $L$, the section ring $R(Y, L)$ is finitely generated.

(2) The $S Q M s f_{i}$ appearing in Definition 1.4 .1 are, up to Mori equivalence, the only SQMs of $Y$.

(3) There are finitely many birational contractions

$$
g_{i}: Y \rightarrow Y_{i}, \quad i=1, \ldots, M,
$$

where the $Y_{i}$ are Mori dream spaces, such that

$$
\overline{\operatorname{Eff}}(Y)=\bigcup_{i=1}^{M} g_{i}^{*}\left(\operatorname{Nef}\left(Y_{i}\right)\right) * \operatorname{exc}\left(g_{i}\right) .
$$

In particular, $\overline{\operatorname{Eff}}(Y)$ is rational polyhedral. Here, $\operatorname{exc}\left(g_{i}\right)$ is the cone spanned by the $g_{i}$-exceptional prime divisors. Up to Mori equivalence, these are all birational contractions of $Y$ with $\mathbb{Q}$-factorial image.

(4) For any divisor $D$ on $Y$ we can run a Mori program. If the divisor becomes nef at some step in the program, it also becomes semiample.

As for the case of SQMs, we can assume the birational contractions $g_{i}$ to be pairwise Mori inequivalent. Clearly, the SQMs $f_{i}$ appear among the $g_{i}$.

Definition 1.4.3. Consider the situation of Theorem 1.4.2. The cones

$$
g_{i}^{*}\left(\operatorname{Nef}\left(Y_{i}\right)\right) * \operatorname{exc}\left(g_{i}\right)
$$

have disjoint interior for inequivalent $g_{i}$, and are called the Mori chambers of $Y$.

REMARK 1.4.4. The decomposition of the effective cone into Mori chambers has the following properties.

(1) The Mori chambers are precisely the closures of the Mori equivalence classes which have non-empty interior. Different Mori chambers can only intersect in their boundaries.

(2) The identity map is a rational contraction, so that

$$
\operatorname{Nef}(X) \subset \overline{\operatorname{Eff}}(X)
$$

is a Mori chamber.

(3) The decomposition of $\overline{\mathrm{Eff}}(X)$ into Mori chambers respects the movable cone. This is because the SQMs $f_{i}$ appear among the birational contractions $g_{i}$.

We close this subsection by a very brief sketch of Mori's program, motivating the naming of Mori dream spaces. We refer to [Mat02] for more details.

REMARK 1.4.5. The final property given in Theorem 1.4.2 is the reason why Mori dream spaces are named that way.

Suppose we are given a divisor $D$ on a variety $X$. The general goal of the 
Mori program is to find a birational model $\left(X^{\prime}, D^{\prime}\right)$ such that the transformed divisor $D^{\prime}$ is nef on $X^{\prime}$. The strategy to construct $X^{\prime}$ is to apply a sequence of contractions, which remove curves $C$ such that

$$
D \cdot C<0 .
$$

In general, it is not known whether the steps of such a program can be carried out, and even if this is possible, whether such a program terminates. But for a Mori dream space $X$, this is possible, and the contractions which are need are parameterized by the cone structure of $\overline{\operatorname{Eff}}(X)$.

\subsubsection{GIT quotients and the Cox ring.}

Suppose that $Y$ is a normal, projective and $\mathbb{Q}$-factorial variety such that $\operatorname{Pic}(Y)_{\mathbb{Q}}=N^{1}(Y)_{\mathbb{Q}}$. Choose a collection of line bundles

$$
L_{1}, \ldots, L_{r} \in \operatorname{Pic}(Y),
$$

which forms a basis of the vector space $N^{1}(Y)_{\mathbb{Q}}$ over $\mathbb{Q}$. The Cox ring of $Y$, with respect to that basis, is defined as

$$
\operatorname{Cox}(Y)=\bigoplus_{a \in \mathbb{Z}^{r}} H^{0}\left(Y, L_{1}^{\otimes a_{1}} \otimes \ldots \otimes L_{r}^{\otimes a_{r}}\right) .
$$

Corresponding to the grading of $\operatorname{Cox}(Y)$, there is the action of the torus $T=$ $T^{r}$ on the Cox ring. Namely, for $t \in T$ and $s$ a section in the homogeneous piece to some $a \in \mathbb{Z}^{r}$ we have

$$
t * s=\left(t_{1}^{a_{1}} \cdot \ldots \cdot t_{r}^{a_{r}}\right) \cdot s .
$$

REMARK 1.4.6. The Cox ring is named after Cox, who used a similar ring for the description of toric varieties (consider [Co95]).

The Cox ring itself depends on the choice of a basis $L_{1}, \ldots, L_{r}$, but the question whether the Cox ring is finitely generated does not.

With a more careful treatment (see [ADHL15]), it is possible to introduce a variant of the Cox ring which does not require the choice of a basis.

Using the Cox ring, one can give an alternative description of Mori dream spaces (consider [HK00], Proposition 2.9).

Theorem 1.4.7. Let $Y$ denote a normal, $\mathbb{Q}$-factorial and projective variety such that

$$
\operatorname{Pic}(Y)_{\mathbb{Q}}=N^{1}(Y)_{\mathbb{Q}}
$$

Then $Y$ is a Mori dream space if and only if $\operatorname{Cox}(Y)$ is a finitely generated $k$-algebra.

The strategy of proof rests on another result of [HK00]. They show that, under sufficiently good properties, a GIT quotient of an affine variety is a Mori dream space. They then show that the action

$$
T^{r} \times \operatorname{Spec}(\operatorname{Cox}(Y)) \rightarrow \operatorname{Spec}(\operatorname{Cox}(Y))
$$

satisfies these properties, and that a GIT quotient recovers $Y$. 
More precisely, they assume the following situation.

Assumption 1.4.8. Let a reductive group $G$ act on an affine variety $X$, and let $\chi_{0} \in \chi(G)$ denote a character such that the following assertions are satisfied.

(1) The general stabilizer $G_{x}$ is finite.

(2) $X$ is normal and $\mathrm{Cl}(X)_{\mathbb{Q}}=0$.

(3) Up to constants, there are no non-trivial $G$-invariant invertible regular functions, i.e.

$$
E(X)^{G}=0 .
$$

(4) All GIT quotients of $X$ are projective.

(5) For GIT chambers, stability and semistability coincides, i.e. Assumption 1.1.18 holds.

(6) $\chi_{0}$ is contained in the interior of a GIT chamber, and the unstable locus is of codimension at least two. That is,

$$
\operatorname{codim}\left(X \backslash X^{\chi_{0}-\text { sst }}\right) \geq 2 \text {. }
$$

The condition $\mathrm{Cl}(X)_{\mathbb{Q}}=0$ implies that $X$ is $\mathbb{Q}$-factorial, and that, up to multiples, all line bundles are trivial. Hence, the $G$-line bundles are of the form $L_{\chi}$ for some character $\chi$.

Theorem 1.4.9. Consider the situation as in Assumption 1.1.18, and denote by

$$
q: X^{\chi_{0}-\text { sst }} \rightarrow Y
$$

the GIT quotient. Then the following assertions hold.

(1) $Y$ is a Mori dream space.

(2) Descent of the linearized bundle $L_{\chi}$ induces a map

$$
\psi: \chi(G)_{\mathbb{Q}} \rightarrow \operatorname{Pic}(Y)_{\mathbb{Q}},
$$

which is an isomorphism.

(3) We have

$$
\psi\left(C^{G}(X)\right)=\overline{\operatorname{Eff}}(Y),
$$

i.e. the G-ample cone is identified with the pseudo-effective cone. Moreover, Mori chambers are identified with GIT chambers, and the GIT chamber containing $\chi_{0}$ is identified with the nef cone.

(4) Every rational contraction

$$
f: Y \rightarrow Y^{\prime}
$$

is induced by VGIT. That is, $Y^{\prime}=Y_{\chi}$ for a suitably chosen character $\chi$, and $f$ is equivalent to the rational map provided by VGIT. 


\subsection{Almost factorial domains}

Let $X=\operatorname{Spec}(A)$ denote an affine variety. In particular, $X$ is supposed to be irreducible, so $A$ is an integral domain. It is well-known that $A$ is a unique factorization domain (UFD) if and only if $X$ is normal and $\mathrm{Cl}(X)=0$.

Following [St67], we call $A$ an almost factorial domain (AFD) if and only if $X$ is normal and $\operatorname{Cl}(X)$ is torsion.

First, we review the relevant properties of and conventions on AFDs, which are established in [St67].

(1) A nonzero nonunit $x \in A$ is called primary if the ideal $(x) \subset A$ is a primary ideal. In that case, the associated prime ideal $\mathfrak{p}_{x}$ is defined as the prime ideal associated to $(x)$.

(2) Two primary elements which have the same associated prime ideal are up to units powers of a third primary element. Conversely, powers of a primary element are again primary.

(3) For a nonzero nonunit $y \in A$, a suitable power $y^{n}$ can be factored into primary elements.

(4) If $D \subset X$ is a prime divisor, there exists a primary element $x \in A$ and a natural number $n$ such that

$$
n D=\operatorname{div}(x) .
$$

Additionally, we need the uniqueness of the set of associated prime ideals in a primary decomposition, which can be proven by a slight variation of the well-known proof that the elements in a decomposition into primes are uniquely determined. We note that the prime ideal associated to a primary ideal is given as its radical ideal.

Lemma 1.5.1. The following statements hold in any integral domain.

(1) Suppose there are two decompositions

$$
a_{1} \cdot \ldots \cdot a_{r}=b_{1} \cdot \ldots \cdot b_{s}
$$

into primary elements. Then the sets of the prime ideals associated to the $a_{i}$ and associated to the $b_{j}$ respectively coincide.

(2) If we have a primary element $x \in A$ such that

$$
x \mid a_{1} \cdot \ldots \cdot a_{r},
$$

then $x \mid a_{i}^{n}$ for a suitable index $i$ and a suitable power $n$.

(3) Given two primary elements $x, y \in A$ such that $x \mid y$, it follows that the associated prime ideals coincide.

Proof. The first assertion easily follows from the second and third assertions.

Induction over $r$ proves the second assertion, where $r=1$ is trivial. If $x \mid a_{1} \cdot \ldots \cdot a_{r-1}$, we are done by the induction hypothesis, and if not we have $a_{r}^{n} \in(x)$ since $x$ is primary.

Under the hypothesis of the third assertion we may write $y=x y^{\prime}$. Now $y \nmid y^{\prime}$, since otherwise $x$ would be a unit, so $y \mid x^{n}$ for some power $n$. Thus 
there are inclusions of ideals

$$
(y) \subset(x) \supset\left(x^{n}\right) \subset(y) .
$$

This yields the inclusions

$$
\mathfrak{p}_{y} \subset \mathfrak{p}_{x}=\mathfrak{p}_{x^{n}} \subset \mathfrak{p}_{y}
$$

by taking the radicals, which finishes the proof. 



\section{CHAPTER 2}

\section{Mori dream spaces}

In this section, we generalize the theorem of $\mathrm{Hu}$ and Keel about GIT quotients to the case where the unstable locus is of codimension one. We apply this result to quiver moduli, and use it to describe quotients of Mori dream spaces.

\subsection{Quotients of spectra of AFDs}

In this first section we establish our main result. We use the properties of an almost factorial domain to describe the influence of unstable codimension components to the GIT chamber structure, and how this is related to Mori chambers of the quotients.

\subsubsection{Divisors under the group action.}

As a first step, we compute the regular invertible functions on an open subset of the spectrum of an AFD. Obviously, this does not require the introduction of a group action yet.

Assumption 2.1.1. Let $A$ denote an AFD, and let $U \subset X=\operatorname{Spec}(A)$ denote an open subset. By

$$
\mathcal{Z}=\mathcal{Z}(U)=\left\{Z_{1}, \ldots, Z_{q}\right\}
$$

we denote the set of irreducible components of $X \backslash U$ which are of codimension one in $X$. Interpreting the elements $Z_{i}$ as prime divisors on the variety $X$, the fact that $A$ is almost factorial implies that $n_{i} Z_{i}=\operatorname{div}\left(g_{i}\right)$ is a principal divisor for some natural number $n_{i}$ and a primary element $g_{i} \in A$ (consider Section 1.5). In particular it holds that $Z_{i}=\mathrm{N}\left(g_{i}\right)$.

In the special case where $A$ is a UFD, we have that every prime divisor is principal, and hence $Z_{i}=\operatorname{div}\left(g_{i}\right)$ for prime elements $g_{i} \in A$.

Lemma 2.1.2. In the situation of Assumption 2.1.1 the map

$$
F: A^{*} \times \mathbb{Z}^{q} \rightarrow \mathcal{O}(U)^{*},
$$

given by

$$
\left.\left(\lambda, a_{1}, \ldots, a_{q}\right) \mapsto \lambda \cdot g_{1}^{a_{1}} \cdot \ldots \cdot g_{q}^{a_{q}}\right|_{U},
$$

is injective with torsion cokernel. In particular, there is an isomorphism

$$
E(X)_{\mathbb{Q}} \times \mathbb{Q}^{q} \rightarrow E(U)_{\mathbb{Q}} .
$$

If $A$ is a UFD, the map $F$ is an isomorphism, and $E(X) \times \mathbb{Z}^{q} \simeq E(U)$.

Proof. First assume that

$$
1=\lambda \cdot g_{1}^{a_{1}} \cdot \ldots \cdot g_{q}^{a_{q}}
$$


on $U$ for some $\left(a_{1}, \ldots, a_{q}\right) \in \mathbb{Z}^{q}$, and $\lambda \in A^{*}$ on $U$. By removing factors with $a_{i}=0$ and bringing factors with $a_{i}<0$ to the other side, we can assume without loss of generality

$$
g_{1}^{a_{1}} \cdot \ldots \cdot g_{s}^{a_{s}}=\lambda \cdot g_{s+1}^{a_{s+1}} \cdot \ldots \cdot g_{r}^{a_{r}}
$$

for some $a_{i}>0$ globally on $X$.

If we assume that this equation is non-trivial, which is $r \neq 0 \neq s$, there exists a point $x \in Z_{1} \backslash\left(Z_{s+1} \cup \ldots \cup Z_{r}\right)$. But then we obtain the contradiction

$$
0=g_{1}(x)^{a_{1}} \cdot \ldots \cdot g_{s}(x)^{a_{s}}=\lambda(x) \cdot g_{s+1}(x)^{a_{s+1}} \cdot \ldots \cdot g_{r}(x)^{a_{r}} \neq 0 .
$$

Thus all $a_{i}$ have to vanish, which also implies $\lambda=1$. This proves the injectivity of the map $F$.

To prove surjectivity (up to torsion in the case where $A$ is an AFD), we may assume without loss of generality that $X \backslash U$ is of pure codimension one, using Hartogs' lemma 1.0.1.

Given $g, h \in \mathcal{O}(U)=\mathcal{O}\left(\mathrm{D}\left(g_{1} \cdot \ldots \cdot g_{q}\right)\right)$, which are inverse to each other, we may write

$$
g=a_{g} /\left(g_{1} \cdot \ldots \cdot g_{q}\right)^{s_{g}}, h=a_{h} /\left(g_{1} \cdot \ldots \cdot g_{q}\right)^{s_{h}}
$$

for some $a_{g}, a_{h} \in A$ and $s_{g}, s_{h} \in \mathbb{N}$, and hence

$$
\left(g_{1} \cdot \ldots \cdot g_{q}\right)^{s_{g}+s_{h}}=a_{g} a_{h} .
$$

First assume that $\mathrm{Cl}(X)=0$. Then, since the $g_{i}$ are irreducible in the unique factorization domain $A$, both $a_{g}$ and $a_{h}$ are products of multiples of the $g_{i}$ up to invertible elements, and are hence contained in the image of $F$, so $g$ is in the image of $F$ as well.

For the general case we choose a power $n$ such that there exist decompositions $a_{g}^{n}=x_{1} \cdot \ldots \cdot x_{s}$ and $a_{h}^{n}=y_{1} \cdot \ldots \cdot y_{t}$ into primary elements. Clearly

$$
\left(g_{1} \cdot \ldots \cdot g_{q}\right)^{n\left(s_{g}+s_{h}\right)}=a_{g}^{n} a_{h}^{n}=x_{1} \cdot \ldots \cdot x_{s} y_{1} \cdot \ldots \cdot y_{t}
$$

are two decompositions of $a_{g}^{n} a_{h}^{n}$ into primary elements. By uniqueness of the associated prime ideals in a primary decomposition (Lemma 1.5.1), the prime ideals associated to primary elements on the right must be contained in the family $\mathfrak{p}_{1}, \ldots, \mathfrak{p}_{r}$. Thus, up to units each of the elements $x_{i}$ is a multiple of a primary element $z_{i}$ such that one of the $g_{j}$ is a multiple of $z_{i}$. Again taking multiples if necessary, this implies that some power of $a_{g}$ is up to units a product of the $g_{i}$, which implies that $g^{N}$ is in the image of $F$ for some power.

Now we introduce a group action. Note that any geometric quotient of the form as below is given as a GIT quotient for a character in the interior of a GIT chamber according to Proposition 2.1.15. 
Assumption 2.1.3. Given the situation of Assumption 2.1.1, we assume furthermore that a reductive group $G$ acts on $X$ such that $U$ is $G$-invariant. Later on, we will further require that there exists a geometric quotient

$$
q: U \rightarrow Y,
$$

and that the stabilizers $G_{x}$ for $x \in U$ are finite. Except for in Proposition 2.1.7, we additionally assume that $Y$ is projective, and hence $E(Y)=0$. All GIT quotients of $X$ are required to be projective, which is implied by $\mathcal{O}(X)^{G}=A^{G}=k$.

We recall that the vanishing order of $g_{i}$ on $Z_{i}=\mathrm{N}\left(g_{i}\right)$ is denoted by $n_{i}$.

LEMma 2.1.4. In the situation of Assumption 2.1.3 the following holds.

(1) The action of $G$ on $X$ induces an action of $G$ on $\mathcal{Z}$, and we denote the set of orbits as

$$
\mathcal{Z} / G=\left\{B_{1}, \ldots, B_{r}\right\} .
$$

If $G$ is connected, this action is trivial.

(2) Suppose that $\mathrm{N}(h) \subset X$ is $G$-invariant for some nonzero nonunit $h \in$ A. We further require that in the primary decomposition

$$
h^{n}=h_{1} \cdot \ldots \cdot h_{s}
$$

the vanishing orders of $h_{i}$ and $h_{j}$ on their respective nullstellensets $\mathrm{N}\left(h_{i}\right)$ and $\mathrm{N}\left(h_{j}\right)$ agree, whenever $\mathrm{N}\left(h_{i}\right)$ and $\mathrm{N}\left(h_{j}\right)$ have the same orbit under the action of $G$ on $\mathcal{Z}(\mathrm{D}(h))$, and that the prime ideals associated to $h_{i}$ and $h_{j}$ differ when $i \neq j$. Then $h^{n}$ is a semiinvariant with respect to a uniquely determined character. Conversely, all semiinvariants are of such a form.

Proof. To prove the first assertion observe that if $G^{\prime} \subset G$ is a connected component, and $Z \in \mathcal{Z}$, then $G^{\prime} * Z \subset X \backslash U$ is irreducible and hence contained in some $Z^{\prime} \in \mathcal{Z}$. By equality of dimensions we have $G^{\prime} * Z=Z^{\prime}$. It is easy to see that this defines an action of $G / G^{0}$ on $\mathcal{Z}$, and hence an action of $G$.

For the second assertion we need some preparations.

By the first assertion, $G$ acts on $\mathcal{Z}(\mathrm{D}(h))=\left\{\mathrm{N}\left(h_{1}\right), \ldots, \mathrm{N}\left(h_{s}\right)\right\}$, and we denote by $\pi_{g}$ the permutation of indices associated to an element $g \in G$. It is immediate to verify that $\mathrm{N}\left(g * h_{i}\right)=\mathrm{N}\left(h_{\pi_{g}(i)}\right)$, so

$$
g * h_{i}=\lambda z^{k} \text { and } h_{\pi_{g}(i)}=\lambda^{\prime} z^{l}
$$

are multiples of a third primary element $z \in A$ up to units $\lambda, \lambda^{\prime} \in A^{*}$.

On the other hand, $g$ can be interpreted as an automorphism of $A$, and hence of $X$, which induces an isomorphism of local rings

$$
\mathcal{O}_{X, \xi_{i}} \rightarrow \mathcal{O}_{X, \xi_{\pi_{g}(i)}}
$$

where $\xi_{i}$ and $\xi_{j}$ are the generic points of $\mathrm{N}\left(h_{i}\right)$ and $\mathrm{N}\left(h_{j}\right)$ in the affine scheme $X$. So $\operatorname{ord}_{\mathrm{N}\left(h_{i}\right)}\left(h_{i}\right)=\operatorname{ord}_{\mathrm{N}\left(h_{\pi_{g}(i)}\right)}\left(g * h_{i}\right)$, from which we deduce $k=l$. In other words, $g$ permutes the elements $h_{i}$ up to units, so that the equation

$$
g * h_{1} \cdot \ldots \cdot h_{s}=\lambda(g) \cdot h_{1} \cdot \ldots \cdot h_{s}
$$


holds for a unit $\lambda(g) \in A$. A suitable power $g^{p}$ is contained in the connected component $G^{0}$ of the identity element, hence the class of $\left.h^{n}\right|_{\mathrm{D}(h)}$ in $E(\mathrm{D}(h))=E(\mathrm{D}(h))^{G^{0}}$ (the equality holds by [KKV 89], Proposition 1.3) is $g^{p}$-invariant. We conclude that $\lambda(g)^{p} \in \mathbb{G}_{m}$, so $\lambda(g)$ is a constant function on $\mathrm{D}(h)$, and hence on $X$, which takes value in $k^{*}$. Finally, it is easy to see that the assignment $g \mapsto \lambda(g) \in \mathbb{G}_{m}$ defines a character of $G$.

Conversely, $h^{n}$ and $g * h^{n}=\chi(g) \cdot h^{n}$ have the same vanishing order on any prime divisor, and the claim follows from the computations above.

For an orbit $B_{i} \in \mathcal{Z} / G$, the group action provides ambient isomorphisms between the divisors $Z_{j} \in B_{i}$. We can thus assume that the defining functions $g_{j}$ have the same vanishing order, i.e.

$$
n_{i} Z_{j}=\operatorname{div}\left(g_{j}\right) .
$$

LEMma 2.1.5. In the situation of Assumption 2.1.3, the following holds.

(1) For any orbit $B_{i} \in \mathcal{Z} / G$, the function

$$
f_{i}=\prod_{Z_{j} \in B_{i}} g_{j}
$$

satisfies the assertions of Lemma 2.1.4.(2), so $g * f_{i}=\chi_{i}(g) \cdot f_{i}$ for a uniquely determined character $\chi_{i} \in \chi(G)$.

(2) The isomorphism $F: E(X)_{\mathbb{Q}} \times \mathbb{Q}^{q} \rightarrow E(U)_{\mathbb{Q}}$ given in Lemma 2.1.2 induces an isomorphism

$$
E(X)_{\mathbb{Q}}^{G} \times \mathbb{Q}^{r} \rightarrow E(U)_{\mathbb{Q}}^{G} .
$$

If $A$ is a UFD, the same statement is true with coefficients in $\mathbb{Z}$.

Proof. The first assertion immediately follows from Lemma 2.1.4. For the second assertion, consider the map $F^{\prime}: E(X)^{G} \times \mathbb{Z}^{r} \rightarrow E(U)$, given by

$$
\left(\lambda, m_{1}, \ldots, m_{r}\right) \mapsto \lambda \cdot f_{1}^{m_{1}} \cdot \ldots \cdot f_{r}^{m_{r}},
$$

where the $f_{i}$ are associated to orbits as in the third assertion. We are done by applying the second assertion of Lemma 2.1.4, and Lemma 2.1.2.

From now on we will continue using the notation as it is introduced in Lemma 2.1.4 and Lemma 2.1.5.

Convention 2.1.6. Let $r$ denote the number of orbits of $\mathcal{Z}(U)$ under the action of $G$. Further, let $f_{i}$ and $\chi_{i}$ denote the associated semiinvariant and character to an orbit $B_{i} \in \mathcal{Z}(U) / G$.

Proposition 2.1.7. In the situation of Assumption 2.1.3, there is an up to torsion exact sequence

$$
0 \rightarrow E(Y) \rightarrow E(X)^{G} \times \mathbb{Z}^{r} \rightarrow \chi(G) \rightarrow \operatorname{Pic}(Y) \rightarrow 0,
$$

which is that this sequence becomes exact after tensoring with $\mathbb{Q}$. In particular, the formula

$$
\rho(Y)=\operatorname{rk}(\chi(G))-\left(|\mathcal{Z} / G|+\operatorname{rk}\left(E(X)^{G}\right)\right)+\operatorname{rk}(E(Y))
$$

for the Picard number holds. If the stabilizers for points on $U$ are trivial, $A$ is a UFD, and if furthermore $G$ is connected, then the above sequence is exact by itself. 
Proof. We want to apply Proposition 5.1 of [KKV89] to our situation. For the remainder of the proof we adopt the notation given there, except that we replace $X$ by $U$.

Because $U$ is open in $X$, the group

$$
\operatorname{Pic}(U) \longleftrightarrow \mathrm{Cl}(U) \longleftarrow \mathrm{Cl}(X)
$$

is torsion, and vanishes if $A$ is a UFD. The cokernel of

$$
q^{*}: \operatorname{Pic}(Y) \rightarrow \operatorname{Pic}^{G}(U)
$$

is a subgroup

$$
\operatorname{coker}\left(q^{*}\right) \subset \prod_{x \in \mathcal{C}} \chi\left(G_{x}\right),
$$

where $\mathcal{C}$ is a finite set of points representing closed orbits. To show that this group is finite, it thus suffices to prove that each $\chi\left(G_{x}\right)$ is finite, which is true since the $G_{x}$ are finite by assumption.

Furthermore, because the group $G / G^{0}$ is finite, the group $H^{1}\left(G / G^{0}, E(U)\right)$ is torsion (consider [W94], Theorem 6.5.8), and vanishes if $G$ is connected.

Up to torsion the diagram thus reduces to an exact sequence

$$
0 \rightarrow E(Y) \rightarrow E(U)^{G} \rightarrow \chi(G) \rightarrow \operatorname{Pic}(Y) \rightarrow 0,
$$

and the claim is implied by Lemma 2.1.5.

\subsubsection{Divisors under descent.}

We now introduce the descent map $\psi$, sending characters to line bundles on the quotient. This map is surjective, and by Lemma 2.1.5, the kernel is spanned by the characters $\chi_{i} \in \chi(G)$ and $\chi^{\prime} \in \chi(G)$ such that

$$
g * f_{i}=\chi_{i}(g) \cdot f_{i}, \text { and } g * f=\chi^{\prime} \cdot f
$$

for $B_{i} \in \mathcal{Z} / G$, and for $f \in E(X)^{G}$.

Definition 2.1.8. The last map in the exact sequence of Proposition 2.1.7 induces a map

$$
\psi: \chi(G)_{\mathbb{Q}} \rightarrow \operatorname{Pic}(Y)_{\mathbb{Q}},
$$

given by sending a character to the descent of the trivial line bundle $L$, linearized by that character.

It is easy to see that multiplication with $\chi^{\prime}$ corresponding to an invertible regular function $f$ does not change the semistable locus. This is not true in general for the characters $\chi_{i}$ associated to $B_{i} \in \mathcal{Z} / G$. Note that the $\chi_{i}$ as in Lemma 2.1.5 can be interpreted as directions in the space $\chi(G)_{\mathbb{Q}}$.

Definition 2.1.9. A GIT class $C \subset \chi(G)$ is called a stable class with respect to $U$ if for all the characters $\chi_{i}$ as in Lemma 2.1.5 we have

$$
\chi_{i} \cdot C \subset C \text {. }
$$


As might be expected, a GIT chamber is stable with respect to itself.

Lemma 2.1.10. Let $\chi \in \chi(G)$ denote a character in the interior of a GIT chamber $C$. Then, $C$ is stable with respect to

$$
U=X^{\chi-s s t} .
$$

Proof. Let $f_{i}$ denote the semiinvariant with respect to the character $\chi_{i}$, associated to some orbit in $\mathcal{Z}(U) / G$ according to Lemma 2.1.5. For a semiinvariant $f$ to some multiple of $\chi$, we have

$$
N\left(f \cdot f_{i}\right)=N(f),
$$

since the vanishing locus of $f_{i}$ is contained in the complement of $U$. Thus,

$$
X^{\chi-\text { sst }} \subset X^{\chi \chi_{i}-\text { sst }},
$$

which implies $\chi_{i} \cdot \chi \in \bar{C}$ by VGIT. But this holds for all $\chi \in C$, so that $\chi_{i} C \subset \bar{C}$. But this is only possible if $C$ remains invariant under translation by $\chi_{i}$.

Because there are only finitely many GIT classes, a ray starting in any character will stay in some fixed class for large distances, and the content of the following lemma is that the rays in the directions of the $\chi_{i}$ satisfy this simultaneously.

LEMma 2.1.11. Using the notation of Assumption 2.1.3, choose an arbitrary character $\chi \in \chi(G)$. Then, after replacing $\chi$ by a suitable multiple, there is an isomorphism of section rings

$$
R\left(X, L_{\chi \chi_{1}^{m_{1}} \ldots \chi_{r}^{m_{r}}}\right)^{G} \rightarrow R\left(U, L_{\chi}\right)^{G},
$$

given in degree $n$ as

$$
\left.s \mapsto \frac{1}{f_{1}^{n m_{1}} \cdot \ldots \cdot f_{r}^{n m_{r}}} s\right|_{U},
$$

if we take the $m_{i}=m_{i}(\chi)$ suitably large. In particular,

$$
\chi \cdot \chi_{1}^{m_{1}} \cdot \ldots \cdot \chi_{r}^{m_{r}}
$$

is contained in a stable class, for $m_{i}$ large enough.

Proof. Injectivity is obvious. Consider the map

$$
\phi: \bigoplus_{m \in \mathbb{N}^{r}, n \geq 0} H^{0}\left(X, L_{\chi^{n} \chi_{1}^{m_{1}} \ldots \chi_{r}^{m_{r}}}\right)^{G} \rightarrow \bigoplus_{n \geq 0} H^{0}\left(U, L_{\chi^{n}}\right)^{G},
$$

given on homogenous elements in a similar form as the map in the statement of the lemma. Note that $\phi$ is graded with respect to the $n$-gradings, and surjective since every section in $H^{0}\left(U, L_{\chi}\right)^{G}$ can be lifted to a global section after multiplication with sufficiently many $g_{i}$.

Note that the characters $\chi_{i}$ are linearly independent by the sequence in Lemma 2.1.7 and the isomorphism in Lemma 2.1.5.(2), so that the left hand side is isomorphic to

$$
H^{0}(X, L)\left[S, T_{1}, T_{2}, \ldots, T_{r}\right]^{G}=A\left[S, T_{1}, T_{2}, \ldots, T_{r}\right]^{G},
$$

where the action of $G$ is given as

$$
g * S=\chi(g)^{-1} S, g * T_{i}=\chi_{i}(g)^{-1} T_{i},
$$


and the action on scalars is inherited from the action of $G$ on $A$. This fixed point algebra is finitely generated as a $k$-algebra by the Theorem of HilbertNagata, so the right hand side is finitely generated as well. Of course it is sufficient to lift the finitely many generators, which after taking a suitable thinning of the section ring can be assumed to all live in degree 1. Taking fixed powers $m_{1}, \ldots, m_{r}$ which lift all generators thus gives a surjective map as claimed.

The following useful observation can be deduced from Lemma 2.1.11.

COROLlary 2.1.12. If $\chi \in \chi(G)$ is contained in a stable class, then

$$
X^{\chi-\text { sst }} \cap U=X^{\chi-\text { sst }}
$$

in codimension one.

Proof. Clearly, the only codimension one components which could contradict the equality are of the form $Z_{i}$. But by Lemma 2.1.11 there are isomorphisms

$$
H^{0}\left(X, L_{\chi \chi_{1}^{m_{1}+1} \ldots \chi_{r}^{m_{r}+1}}\right)^{G} \rightarrow H^{0}\left(U, L_{\chi}\right)^{G} \leftarrow H^{0}\left(X, L_{\chi \chi_{1}^{m_{1}} \ldots \chi_{r}^{m_{r}}}\right)^{G}
$$

for sufficiently large $m_{i}$, and thus we can assume without loss of generality, replacing $\chi$ by $\chi \cdot \chi_{1}^{m_{1}} \cdot \ldots \cdot \chi_{r}^{m_{r}}$, that any semiinvariant with respect to $\chi$ can be divided by $f_{1}, \ldots, f_{r}$. So the $Z_{i}$ are unstable with respect to $\chi$.

Another consequence of Lemma 2.1.11 is the following.

Lemma 2.1.13. For a character $\chi \in \chi(G)$ contained in a stable class, the canonical map

$$
R\left(X, L_{\chi}\right)^{G} \rightarrow R(Y, \psi(\chi))
$$

is an isomorphism, where we may have to replace $\chi$ by a suitable multiple. In particular, the pseudoeffective cone $\overline{\operatorname{Eff}}(Y)$ is exactly the image of the union of all stable $G$-ample classes.

Proof. By definition of descent we have an induced isomorphism

$$
q^{*}: R(Y, \psi(\chi)) \rightarrow R\left(U, L_{\chi}\right)^{G} .
$$

Taking suitable multiples and multiplying with suitable powers of the $\chi_{i}$, where the latter operation does not change $\psi(\chi)$, the right hand side is isomorphic to $R\left(X, L_{\chi}\right)^{G}$ by Lemma 2.1.11.

Hence, the divisor $\psi(\chi)$ admits a section if and only if $L_{\chi}$ admits a section, which is equivalent to $X^{\chi-\text { sst }} \neq \emptyset$ up to multiples of $\chi$.

Lemma 2.1.14. Let $\chi \in \mathcal{C}$ and $\chi^{\prime} \in \mathcal{C}^{\prime}$ denote two characters in the interior of GIT chambers which are stable with respect to $U$ such that $f_{\chi}$ and $f_{\chi^{\prime}}$ are Mori equivalent. Then

$$
X^{\chi-\text { sst }}=X^{\chi^{\prime}-\text { sst }}
$$

in codimension one.

Proof. We proceed as in the proof of Lemma 5.2 of [ST16], up to the point where

$$
X^{\chi-\text { sst }} \cap U=X^{\chi^{\prime}-\text { sst }} \cap U
$$

in codimension one. Corollary 2.1.12 now implies the desired result. 
We now have established the results needed to show that all subsets and quotients of the form as in Assumption 2.1.3 are induced by GIT.

Proposition 2.1.15. Suppose that a reductive group $G$ acts on the spectrum $X$ of an almost factorial domain $A$. Furthermore, assume that there exists an open $G$-invariant subset $U \subset X$ and a geometric quotient

$$
q: U \rightarrow Y,
$$

where $Y$ is projective. We then claim that $U=X^{\chi \text {-sst }}$ for a character $\chi \in \chi(G)$ in the interior of a GIT chamber.

Proof. We adapt the classical proof of a similar result in the smooth case ([MFK94] Converse 1.13) to our situation. Fix an ample line bundle $A \in$ $\operatorname{Pic}(Y)$ and consider the pullback

$$
q^{*}(A) \in \operatorname{Pic}^{G}(U) .
$$

Up to multiples, $q^{*}(A)$ is trivial as a line bundle, hence the action of $G$ on $q^{*}(A)$ is given by global multiplication with a cocycle. By the proof of Proposition 2.1.7 such cocycles are given by characters, so

$$
q^{*}(A)=\left.\left(L_{\chi}\right)\right|_{U}
$$

for some character $\chi \in \chi(G)$. Furthermore, the isomorphism

$$
\phi:\left.\left.\left(L_{\chi}\right)\right|_{U} \rightarrow\left(L_{\chi \chi_{i}}\right)\right|_{U}, \quad(x, v) \mapsto\left(x, f_{i}^{-1}(x) v\right),
$$

where we use the notation of Lemma 2.1.5, shows that we can assume $\chi$ to be stable with respect to $U$. Then, by an argument as in Corollary 2.1.12, we see that the codimension one components of $X \backslash U$ are unstable with respect to $\chi$.

The original proof of Mumford furthermore employs the fact that the complement of an open affine subset is of pure codimension one. This carries over to our case. Indeed, let $V \subset X$ denote an open affine subset, and denote by $Z \subset X$ the union of the codimension one components of the complement. Up to multiples, $Z$ is a prime divisor, so that $X \backslash Z$ is a principal affine open subset. The map

$$
\mathcal{O}(X \backslash Z) \rightarrow \mathcal{O}(V)
$$

is an isomorphism by Hartogs' lemma 1.0.1, so that these two subsets coincide.

Now that these facts are established, the proof of [MFK94] provides us with the assertion $U \subset X^{\chi-s t}$. Hence we have a diagram

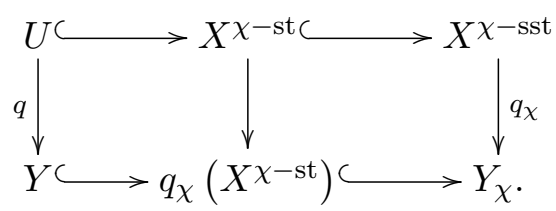

Since $Y$ is projective and $q_{\chi}\left(X^{\chi-s t}\right)$ is irreducible, the open immersion

$$
Y \hookrightarrow q_{\chi}\left(X^{\chi-\mathrm{st}}\right)
$$


is an isomorphism, and the same reasoning applies to the lower right arrow of the diagram. Because geometric quotients parametrize all orbits, this implies that $U$ and $X^{\chi-\text { st }}$ consist of the same orbits. For any orbit

$$
\mathcal{O} \subset X^{\chi-\text { sst }}
$$

the fiber of $q_{\chi}(\mathcal{O})$ must also contain a stable orbit $\mathcal{O}^{\prime}$ by the lower right isomorphism. By the properties of a good quotient, the closures of $\mathcal{O}$ and $\mathcal{O}^{\prime}$ must hence intersect, but $\mathcal{O}^{\prime}$ is closed and of maximal dimension among the orbits, so that the orbits coincide.

In other words, the maps in the upper row are identities as well.

\subsubsection{The main theorem.}

We are finally ready to prove the main result of this section. While the general outline of the proof follows that of the original proof in [HK00], our proof is much more detailed. This is because the presence of codimension one components presents us with several subtle problems, which have to be solved by applying the results discussed in the preceding subsections.

We assume the situation of Assumption 2.1.3. In particular, we have the action of a reductive group $G$ on an affine variety $X$ which is the spectrum of an AFD. We assume that all GIT quotients are projective, and the existence of a geometric quotient

$$
q: U \rightarrow Y
$$

to a projective variety. As Proposition 2.1.15 shows, there is no harm in assuming that $q$ is the GIT quotient associated to a character $\chi_{0}$ in the interior of a GIT chamber $C$. Also recall our general Assumption 1.1.18 about GIT chambers.

TheOrem 2.1.16. In the situation of Assumption 2.1.3 the following holds.

(1) Section rings of divisors on $Y$ are finitely generated, the rational maps associated to stable characters are contractions of $Y$, and every rational contraction to a normal, projective variety is of such a form.

(2) $Y$ is a Mori dream space.

(3) Under the canonical map

$$
\psi: \chi(G)_{\mathbb{Q}} \rightarrow \operatorname{Pic}(Y)_{\mathbb{Q}},
$$

the image of the $G$-ample stable GIT classes is exactly the pseudoeffective cone of $Y$.

(4) Mori chambers of $Y$ are identified with stable GIT chambers, and $C$ is identified with $\operatorname{Nef}(Y)$.

PROOF. It is well-known that a quotient of a normal variety is again normal. To ensure the $\mathbb{Q}$-factoriality of $Y$, we apply Lemma 2.1 of [HK00]. Furthermore, since we assume that stability and semistability with respect to chambers coincide, the chamber quotients are geometric, and hence normality and $\mathbb{Q}$-factoriality for them holds by the same argument. 
For discussing contractions, it suffices to consider the contractions associated to divisors, because all rational contractions are induced in such a way (consider [HK00], Lemma 1.6). Further, since $\psi$ is surjective, we can write any divisor on $Y$ as $D=\psi(\chi)$ up to multiples. By Lemma 2.1.11 we can additionally assume that $\chi$ is contained in a stable class, so that the canonical map

$$
R\left(X, L_{\chi}\right)^{G} \rightarrow R(Y, \psi(\chi))
$$

is an isomorphism by Lemma 2.1.13. Consider the induced isomorphism

$$
\phi: Y_{\chi}=\operatorname{Proj}\left(R\left(X, L_{\chi}\right)^{G}\right) \rightarrow \operatorname{Proj}(R(Y, D)),
$$

and the following diagram

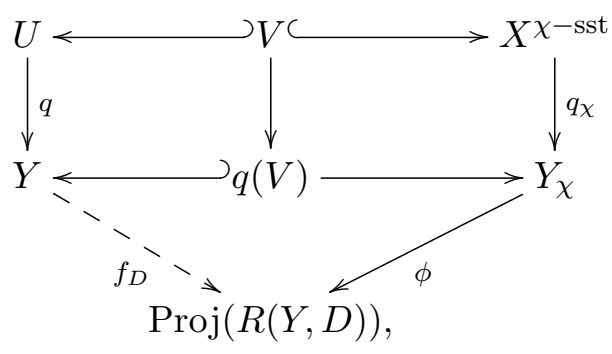

where again $V=U \cap X^{\chi-\text { sst }}$. Note that the two squares at the top form the diagram used in the construction of the rational map $f_{\chi}: Y \rightarrow Y_{\chi}$ (see section 1.1.3). Commutativity is clear except for the part involving $f_{D}$ and the isomorphism $\phi$.

If we choose generators $s_{0}, \ldots s_{d}$ for $R\left(X, L_{\chi}\right)^{G}$ and $s_{0}^{\prime}, \ldots, s_{d}^{\prime}$ for $R(Y, D)$ compatible with $\phi$, and without loss of generality of degree 1 , then the GIT quotient $q_{\chi}$ is given as

$$
x \mapsto\left[s_{0}(x): \ldots: s_{d}(x)\right],
$$

and a similar description holds for $f_{D}$. Hence $\left.f_{D} \circ q\right|_{V}=\left.\phi \circ q_{\chi}\right|_{V}$ and using the surjectivity of the quotient map $V \rightarrow q(V)$, this implies that the lower triangle commutes as well. This proves the first assertion.

If $\chi$ and $\chi^{\prime}$ are characters corresponding to the same stable class, then by a similar argument as above $\psi(\chi)$ and $\psi\left(\chi^{\prime}\right)$ are Mori equivalent.

Since there are only finitely many GIT chambers, which are rational polyhedral, this implies that there are only finitely many Mori chambers as well, which are rational polyhedral as well.

Now, assume conversely that $D$ and $D^{\prime}$ are general elements of the interior of the same Mori chamber. We can again write $D=\psi(\chi)$ and $D^{\prime}=\psi\left(\chi^{\prime}\right)$ for characters $\chi, \chi^{\prime} \in \chi(G)$ in stable GIT chambers, considered to be in the interior. We want to prove that $\chi$ and $\chi^{\prime}$ are GIT equivalent, for which it is sufficient to show $X^{\chi-\text { sst }} \subset X^{\chi^{\prime}-\text { sst }}$. 
Consider the following diagram, where $V=X^{\chi-\text { sst }} \cap X^{\chi^{\prime}-\text { sst }}$.

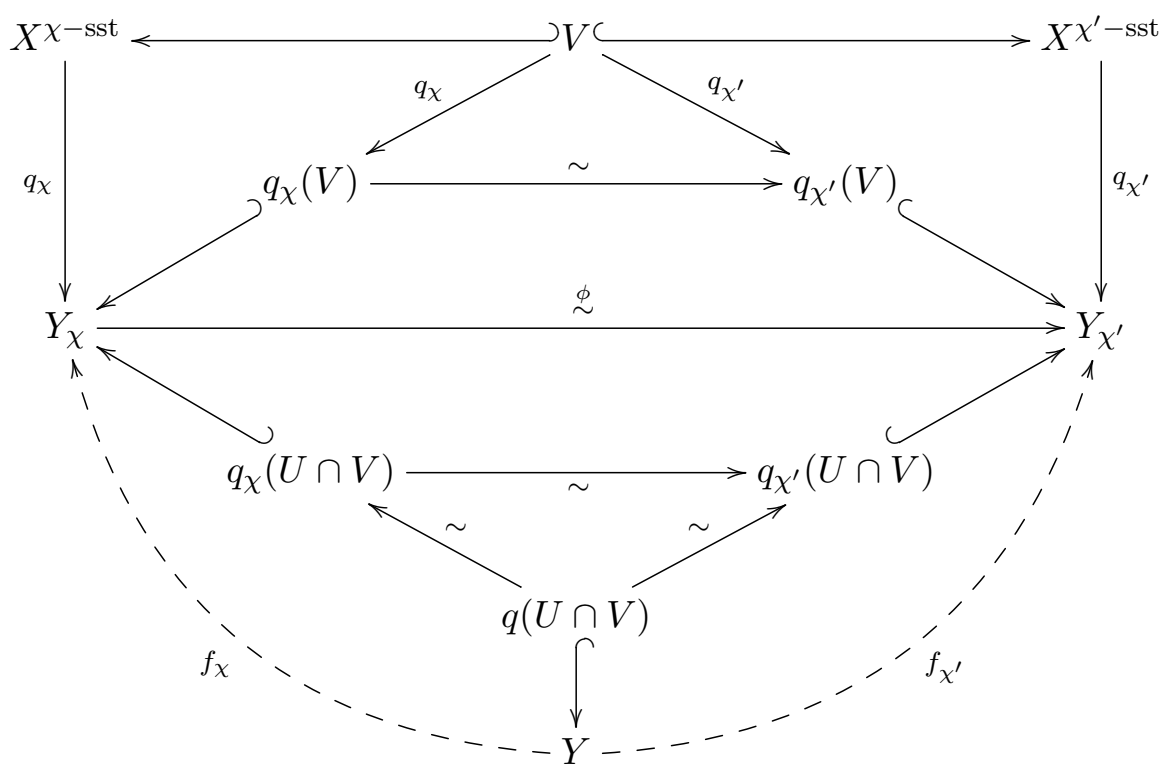

Note that $q(U \cap V)=q\left(U \cap X^{\chi-\text { sst }}\right) \cap q\left(U \cap X^{\chi^{\prime}-\text { sst }}\right)$ is an open subset, where both contractions $f_{\chi}, f_{\chi^{\prime}}$ are defined. For the commutativity of the square involving $\phi$ and the isomorphism $q_{\chi}(V) \simeq q_{\chi^{\prime}}(V)$, note that both maps coincide on the open subset $q_{\chi}(U \cap V)$. The commutativity of the other squares is immediately clear by construction.

Pick any point $x \in X^{\chi-\text { sst }}$.

By the GIT-construction, we have the following for any point $y \in Y_{\chi^{\prime}}$ : there exists a section $s \in H^{0}\left(X, L_{\chi^{\prime}}\right)^{G}$ such that $\left.s\right|_{X \chi^{\prime} \text {-sst }}=q_{\chi^{\prime}}^{*}\left(s^{\prime}\right)$, where $s^{\prime} \in H^{0}\left(Y_{\chi^{\prime}}, A^{\prime}\right)$ is a section of the ample line bundle $A^{\prime}$ on $Y_{\chi^{\prime}}$, given as the descent of the bundle $\left.\left(L_{\chi^{\prime}}\right)\right|_{X \chi^{\prime} \text {-sst }}$, such that $s^{\prime}(y) \neq 0$. In particular, this holds for $y=\left(\phi \circ q_{\chi}\right)(x) \in Y_{\chi^{\prime}}$.

Via $\phi$ the ample line bundle $A^{\prime}$ can be identified with an ample line bundle $A$ on $Y_{\chi}$, and $s^{\prime}$ with a section $s^{\prime \prime}$, and working through the diagram it is easy to check that

$$
\left.\left(L_{\chi^{\prime}}\right)\right|_{V} \simeq q_{\chi}^{*}\left(\left.A\right|_{q_{\chi}(V)}\right) .
$$

A similar statement holds for $s$ and $s^{\prime \prime}$. We thus have a diagram

$$
\begin{gathered}
H^{0}\left(X^{\chi-\text { sst }}, L_{\chi^{\prime}}\right)^{G C} \longrightarrow H^{0}\left(V, L_{\chi^{\prime}}\right)^{G} \\
\downarrow \sim \\
H^{0}\left(X^{\chi-\text { sst }}, q_{\chi}^{*}(A)\right)^{G C} \longrightarrow H^{0}\left(V, q_{\chi}^{*}(A)\right)^{G} .
\end{gathered}
$$

By Lemma 2.1.14, we have $X^{\chi-\text { sst }}=X^{\chi^{\prime}-\text { sst }}$ in codimension one, so by Hartogs' lemma 1.0.1 the map in the first line is an isomorphism.

This finally gives an identification

$$
\left.s\right|_{X \chi-\mathrm{sst}}=q_{\chi}^{*}\left(s^{\prime \prime}\right) \in H^{0}\left(X^{\chi-\mathrm{sst}}, L_{\chi^{\prime}}\right)^{G},
$$


and thus $s(x)=s^{\prime \prime}\left(q_{\chi}(x)\right)=s^{\prime}\left(\phi \circ q_{\chi}(x)\right) \neq 0$, so $x$ is semistable with respect to $\chi^{\prime}$ as desired. This proves the third assertion.

For the second assertion it remains to check that the Mori chambers constituting the moving cone are spanned by divisors which are pullbacks of semiample divisors under SQM's $f_{i}: Y \rightarrow Y_{i}$, and that the nef cones of the $Y_{i}$ are spanned by finitely many semiample bundles (note that $i d: Y \rightarrow Y$ is a SQM as well).

We first observe that the pullback $f^{*}(D)$ of a semiample divisor $D$ under a SQM $f: Y \rightarrow Y^{\prime}$ is movable. Indeed, this is even well-known for the pullback of movable divisors.

Conversely, assume that $D$ is a movable divisor which is contained in the interior of some Mori chamber. Then $f_{D}$ is an isomorphism outside the union of the stable base locus with the augmented base locus ([BCL14], Theorem A). A small perturbation $L-\frac{1}{m} A$ by an ample divisor $A$ does not leave the Mori chamber, and consequently both divisors are given by characters inside the same stable GIT chamber. Obviously, GIT equivalent divisors descend to line bundles with the same stable base locus (by lifting and descending sections), from which we deduce that the augmented base locus of $D$ agrees with its stable base locus

$$
\mathrm{B}_{+}(D)=\mathrm{B}(D) .
$$

This implies that $f_{D}$ is a SQM. By Lemma 1.6 in [HK00], it follows that $D$ is the pullback of an ample divisor under the SQM $f_{D}$.

This proves that $\overline{\operatorname{Mov}}(Y)$ is the union of the cones $f_{i}^{*}\left(\operatorname{Nef}\left(Y_{i}\right)\right)$, and we only need to establish that the cones $\operatorname{Nef}\left(Y_{i}\right)$ are spanned by semiample bundles.

We claim that it suffices to show that $\operatorname{Nef}(Y)$ is spanned by semiample line bundles. Indeed, as we have shown already, the $Y_{i}$ are themselves GIT quotients of $X$ with respect to a character in the interior of a GIT chamber. But all steps of the proof up to now can be carried out for these quotients in parallel, where the stable GIT chamber structure has to be changed accordingly.

Now, let $D \in \operatorname{Nef}(Y)$ denote any divisor, which we write as $D=\psi(\chi)$ for a character $\chi \in C^{\prime}$, where $C^{\prime}$ is a stable GIT class with respect to $U$. This class is contained in the closure of the GIT chamber $C \subset \chi(T)_{\mathbb{Q}}$, with respect to which $Y$ is the GIT quotient. Hence, by VGIT we know

$$
U=X^{\chi_{0}-\text { sst }} \subset X^{\chi-\text { sst }},
$$

ie. for any point in $U$, there exists a semiinvariant with respect to a multiple of $\chi$ which does not vanish in it. For any point $y \in Y$, choose a preimage $x \in U$ and some semiinvariant $f \in H^{0}\left(X, L_{\chi^{m}}\right)^{G}$, which does not vanish in $x$. Then, using Lemma 2.1.13, we know

$$
R\left(X, L_{\chi}\right)^{G} \simeq R(Y, \psi(\chi)),
$$

so that this semiinvariant descends to a section of $m D$ which does not vanish in $y$. This shows that $D$ is semiample, and we are done. 
REMARK 2.1.17. In the situation of Assumption 2.1.3, choose any $G$-ample character $\chi$. In any case we can find a GIT chamber $C$ such that $\chi$ is contained in its closure.

The quotient $Y$ associated to the chamber is a Mori dream space by Theorem 2.1.16, and still $Y_{\chi}$ is normal and projective but may fail to be $\mathbb{Q}$-factorial. As the construction shows, the rational contraction

$$
Y \rightarrow Y_{\chi}
$$

induced by GIT is a regular contraction, so $Y_{\chi}$ is at least a not necessarily $\mathbb{Q}$-factorial Mori dream space by the result of [016], Section 10.1.

Apart from an upper bound on the Picard number, and the assertion that the Mori chamber structure of $Y_{\chi}$ is a coarsening of the Mori chamber structure of $Y$, this observation does not seem to provide any quantitative results, and the qualitative result is already covered by [Ba11]. Even though this does not seem to be helpful in itself, it sparks the hope that the results of this section might possibly extend to good quotients.

REMARK 2.1.18. Another natural approach to extend $\mathrm{Hu}$ and Keel's result to the case where the unstable locus has codimension one works as follows. Suppose that we have the action of a group $G$ on an affine variety $X$, satisfying the assumptions of [HK00]. Let $U$ denote the semistable locus associated to a character $\chi_{0}$ in the interior of a GIT chamber, but where the unstable locus is of codimension one. Then the variety $X^{\prime}$ as in

$$
U \subset X^{\prime}=X \backslash Z,
$$

where $Z$ is the union of the components in $\mathcal{Z}(U)$, is again affine, normal and has $\operatorname{Cl}\left(X^{\prime}\right)_{\mathbb{Q}}=0$. For the induced action of $G$ on $X^{\prime}$ we have

$$
\left(X^{\prime}\right)^{\chi_{0}-\text { sst }}=X^{\chi_{0}-\text { sst }},
$$

so that $X^{\prime}$ additionally satisfies the codimension assumption of $\mathrm{Hu}$ and Keel. It then seems tempting, to apply $\mathrm{Hu}$ and Keel's result to $X^{\prime}$ instead of $X$.

However, the GIT chamber structure for the action of $G$ on $X^{\prime}$ differs from the chamber structure on $X$. To describe how they are related, one has to use arguments similar to those of the preceding subsections.

Furthermore, $X^{\prime}$ acquires additional invertible regular functions. Such functions yield a kernel for the descent map

$$
\psi: \chi(G)_{\mathbb{Q}} \rightarrow \operatorname{Pic}(Y)_{\mathbb{Q}},
$$

where $Y$ denotes the quotient. Hence, the theorem of [HK00] can not be applied directly.

On the other hand, this discussion shows that morally, invertible functions and codimension one components in the unstable locus are the same, at least with respect to the application of our result about quotients. 


\subsection{Quotients of Mori dream spaces}

Let $Y$ denote a Mori dream space, acted upon by a reductive group $G$, which we here assume to be connected. Again we assume that stability and semistability with respect to chambers coincide (consider Assumption 1.1.18). Suppose that there is a $G$-invariant open subset $V \subset Y$ admitting a geometric quotient

$$
q^{\prime}: V \rightarrow Z,
$$

where $Z$ is projective and stabilizers on $V$ are finite. We want to show in this section that $Z$ is again a Mori dream space, and compute the Picard number.

Again, by Remark 2.2.5 given below, such a quotient is given as a GIT quotient with respect to the interior of a chamber. But the proofs occurring in this section do not depend on this observation.

Recall that $Y$ admits the description as a GIT quotient via its Cox ring. Let $r=\rho(Y)$ denote the Picard number of $Y$, and choose line bundles

$$
D_{1}, \ldots, D_{r} \in \operatorname{Pic}(Y),
$$

which form a $\mathbb{Q}$-basis of $\operatorname{Pic}(Y)_{\mathbb{Q}}$. Without loss of generality, we can choose this basis such that $\overline{\operatorname{Eff}}(Y)$ is contained in the convex cone spanned by it. The Cox ring

$$
\operatorname{Cox}(Y)=\bigoplus_{m \in \mathbb{N}^{r}} H^{0}\left(Y, D_{1}^{m_{1}} \otimes \ldots \otimes D_{r}^{m_{r}}\right)
$$

is finitely generated as a $k$-algebra, and there is a quotient representation

$$
q^{\prime \prime}: U \rightarrow Y,
$$

where $U \subset X=\operatorname{Spec}(\operatorname{Cox}(Y))$ is the stable and semistable locus for some character $\chi_{0} \in \chi(T)$ in the interior of a GIT chamber, where $T$ is the torus acting on $\operatorname{Cox}(Y)$ via the grading. The codimension of $X \backslash U$ is greater or equal to two.

REMARK 2.2.1. It is easy to see that

$$
E(X)=\operatorname{Cox}(Y)^{*} / k^{*}=0
$$

using the projectivity of $Y$ and the $\mathbb{N}^{r}$-grading of $\operatorname{Cox}(Y)$ (compare with Corollary 2.2 in [A09]).

A lifting of the action $\mu_{G}: G \times Y \rightarrow Y$ is defined to be an action

$$
\mu_{G}^{\prime}: G \times X \rightarrow X
$$

such that $U$ is $G$-invariant, and such that the actions of $T$ and $G$ on $X$ commute. Obviously, this is equivalent to an action of the product group $T \times G$ on $X$.

As a first step, we ensure the existence of a lifting to a suitably chosen Cox ring. Additionally, we establish that we may take the Cox ring to be an AFD. Note that if $\operatorname{Pic}(Y)$ is torsion-free we may even assume $\operatorname{Cox}(Y)$ to be a UFD (consider [A 09]). 
Lemma 2.2.2. There exists a basis $D_{1}, \ldots, D_{r}$ of $\operatorname{Pic}(Y)_{\mathbb{Q}}$ such that the following assertions hold.

(1) The Cox ring with respect to this basis is almost factorial.

(2) There exists a lifting of the action $\mu_{G}: G \times Y \rightarrow Y$ to $X$.

Proof. We start with any basis $D_{1}, \ldots, D_{r} \in \operatorname{Pic}(Y)$ of $\operatorname{Pic}(Y)_{\mathbb{Q}}$ such that $\overline{\mathrm{Eff}}(Y)$ is contained in the cone spanned by these bundles.

The proof of [HK] Proposition 2.9 shows that $T$ acts freely on $U$ if we replace the $D_{i}$ with sufficiently high powers. In the terminology of Jow (see $[\mathrm{J}]$, Theorem 1.8) such a basis is called a preferred basis, and the associated Cox ring is normal ([J], Proposition 1.12).

We again consider the commutative diagram given in $[\mathrm{KKV}]$ Proposition 5.1, where $X$ in the notation of [KKV] corresponds to $U, G$ to $T$, and $X / / G$ to $Y$. By normality of $U$, and because $T$ is a torus, we know that any line bundle admits a linearization ([KKLV89], Proposition 2.4).

Using Hartogs' lemma 1.0.1, the group $E(U) \simeq E(X)$ vanishes by the Remark 2.2.1 above. Further, $H^{1}\left(T / T_{0}, E(U)\right)$ vanishes since $T$ is connected, and $\prod_{x \in \mathcal{C}} \chi\left(T_{x}\right)$ vanishes because the action of $T$ on $U$ is free. The diagram thus induces an exact sequence

$$
0 \rightarrow \chi(T) \rightarrow \operatorname{Pic}(Y) \rightarrow \operatorname{Pic}(U) \rightarrow 0,
$$

where $\chi(T)$ and $\operatorname{Pic}(Y)$ are both of rank $r$. Since $U$ is $\mathbb{Q}$-factorial ([HK] Lemma 2.1), this implies the first result.

To see the second assertion we fix a $G$-linearization for each bundle $D_{i}$. These linearizations induce a linearization of each product

$$
D^{a}=D_{1}^{a_{1}} \otimes \ldots \otimes D_{r}^{a_{r}}
$$

for $a \in \mathbb{N}^{r}$. Note that the linearization of $D^{a}$ is uniquely determined by the linearizations of the $D_{i}$ since the decomposition of $D^{a}$ as a product of the $D_{i}$ is unique. This gives an action of $G$ on $H^{0}\left(Y, D^{a}\right)$, and thus on $\operatorname{Cox}(Y)$ and $X$ as well.

Recall that the action of $T=\operatorname{Hom}\left(\mathbb{Z}^{r}, \mathbb{G}_{m}\right)$ on $\operatorname{Cox}(Y)$ is given as

$$
t * s=t(a) \cdot s
$$

for $t \in T$ and $s \in H^{0}\left(Y, D^{a}\right)$ homogenous of multidegree $a \in \mathbb{N}^{r}$. Since the action of $G$ is given by a linear action on the fibres of $D^{a}$ these two actions commute. The commuting actions of $G$ and $T$ on $X$ induce commuting contragredient actions on $H^{0}(X, L)=\mathcal{O}(X)$. Hence, if $f \in H^{0}\left(X, L_{\chi}\right)^{T}$ is a $T$-eigenfunction with respect to some character $\chi \in \chi(T)$, which does not vanish in a point $x \in X$, then $g * f$ for $g \in G$ is again a $T$-eigenfunction with respect to $\chi$, which does not vanish in the point $g * x$.

This implies that the semistable locus with respect to any character $\chi$ of $T$ is $G$-invariant; in particular this holds for $U$. 
REMARK 2.2.3. If $G$ is not connected, the proof of Lemma 2.2.2 applies in case there exists a basis $D_{1}, \ldots, D_{r}$ of $\operatorname{Pic}(Y)_{\mathbb{Q}}$ which consists of $G$-invariant line bundles. However such a basis might not exist. The obvious action of $\mathbb{Z}_{2}$ on $\mathbb{P}^{1} \times \mathbb{P}^{1}$ gives a counterexample (as explained in [Br14], Example $1.16)$.

Fixing a basis which satisfies the assertions of the lemma above, we obtain the following result. The proof is straightforward.

Lemma 2.2.4. Let $V \subset Y$ denote a $G$-invariant open subset such that there exists a good quotient

$$
q^{\prime}: V \rightarrow Z \text {. }
$$

Then the composition

$$
q:\left(q^{\prime \prime}\right)^{-1}(V) \stackrel{q^{\prime \prime}}{\rightarrow} V \stackrel{q^{\prime}}{\rightarrow} Z
$$

is a good quotient with respect to the action of $T \times G$ on $X$. If $q^{\prime}$ is geometric, so is the composition.

REMARK 2.2.5. A similar assertion as in Proposition 2.1.15 holds if we replace $X$ by a Mori dream space $Y$. That is, any geometric quotient

$$
q^{\prime}: V \rightarrow Z
$$

of an open and $G$-invariant subset $V \subset Y$ such that $Z$ is projective, is given as the GIT quotient of $X=\operatorname{Spec}(\operatorname{Cox}(Y))$ associated to a GIT chamber.

Proof. Replacing the notation $q: U \rightarrow Y$ in the proof of Proposition 2.1.15 by the geometric quotient

$$
q^{\prime}: V \rightarrow Z,
$$

we get by a similar argument, that $q^{-1}(V) \rightarrow Z$, where $q$ is given as in Lemma 2.2.4 and the preceding construction, is a geometric quotient. Using the descent properties of the quotient $q^{\prime \prime}: U \rightarrow Y$, with notation again as in Lemma 2.2.4, we can thus lift the pullback $\left(q^{\prime}\right)^{*}(A) \in \operatorname{Pic}^{G}(V)$ of an ample line bundle on $Z$ to a $G$-line bundle on $Y$, and the proof of Proposition 2.1.15 applies.

We are now able to prove the main result of this section.

Theorem 2.2.6. In the situation above, $Z$ is a Mori dream space with Picard number

$$
\rho(Z)=\rho(Y)+\operatorname{rk}(\chi(G))-|\mathcal{Z}(V)| .
$$

If $\operatorname{Pic}(Y)$ is torsion-free, and stabilizers of points in $V$ are trivial, the group $\operatorname{Pic}(Z)$ is torsion-free as well. Furthermore, the Mori chamber structure of $Z$ is given by the GIT chambers for the action of $T \times G$ on $X$ which are stable with respect to $\left(q^{\prime \prime}\right)^{-1}(V)$.

Proof. Using the Lemmata 2.2.2 and 2.2.4 we can write $Z$ as a geometric quotient of the open set $U^{\prime}=\left(q^{\prime \prime}\right)^{-1}(V)$. By the codimension assertion on the unstable locus with respect to $\chi_{0}$, the codimension one components of $X \backslash U^{\prime}$ in $X$ are in one-to-one correspondence under $q^{\prime \prime}$ with the codimension one components of $Y \backslash V$. 
Hence, $Z$ is a Mori dream space by Theorem 2.1.16, with Picard number

$$
\rho(Z)=\operatorname{rk}(\chi(T \times G))-|\mathcal{Z}(V)| .
$$

The claimed formula follows using

$$
\begin{aligned}
\chi(T \times G) & =\chi(T) \times \chi(G), \\
\operatorname{rk}(\chi(T)) & =\operatorname{rk}(T)=\rho(Y) .
\end{aligned}
$$

Under the additional assumptions we see that $T \times G$ is connected, $X$ is the spectrum of a UFD, and that stabilizers for the action of $T \times G$ on $\left(q^{\prime \prime}\right)^{-1}(V)$ are trivial, so the torsion-freeness of $\operatorname{Pic}(Z)$ follows.

Finally, by Theorem 2.1.16, we know that Mori chambers of $Z$ are identified with stable GIT chambers in $\chi(T \times G)_{\mathbb{Q}}$.

REMARK 2.2.7. It seems desirable to describe the Mori chambers of $Z$ in terms of the Mori chambers of $Y$. Using the notation of the proof of Theorem 2.2.6, we could try to apply the functor $\operatorname{Pic}^{*}(*) \otimes \mathbb{Q}$ (consider Lemma 1.1.4) to the diagram

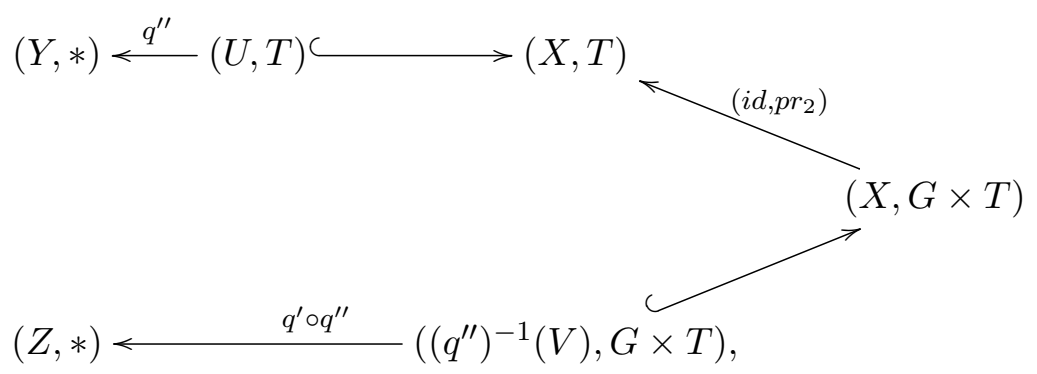

to obtain

$$
\operatorname{Pic}(Y)_{\mathbb{Q}} \simeq \chi(T)_{\mathbb{Q}} \hookrightarrow \chi(G \times T)_{\mathbb{Q}} \rightarrow \operatorname{Pic}(Z)_{\mathbb{Q}} .
$$

One would expect that the inclusion $\chi(T) \subset \chi(T \times G)$, or the projection $\chi(T \times G) \rightarrow \chi(T)$, respects the GIT chambers, though a proof remains elusive. The difficulty seems to be imposed by the change of the group action. For example, we could attempt to use the Hilbert-Mumford-criterion.

Recall that this criterion tells us that a point $x \in X$ is $\chi$-semistable if and only if for all one-parameter subgroups $\lambda$ such that the limit

$$
\lim _{s \rightarrow 0} \lambda(s) * x
$$

exists, the numerical criterion

$$
\langle\lambda, \chi\rangle \geq 0
$$

holds. Here, $\langle *, *\rangle$ denotes the canonical pairing between characters and one-parameter-subgroups. While the numerical side poses no problems, it seems difficult to relate the existence of limits for a group $\lambda: \mathbb{G}_{m} \rightarrow T \times G$ to the existence of limits for the group $\lambda_{T}: \mathbb{G}_{m} \rightarrow T$. 
There is a natural construction of Mori dream subspaces.

Remark 2.2.8. Let $Y$ denote a Mori dream space, and consider the description as a GIT quotient

$$
q: U \rightarrow Y
$$

with respect to the action of the torus $T$, where $U \subset X=\operatorname{Spec}(\operatorname{Cox}(Y))$. Suppose that we have a closed subset $X^{\prime} \subset X$ which is invariant under the action of $T$, and which is the spectrum of an AFD as well. Then, there is a diagram

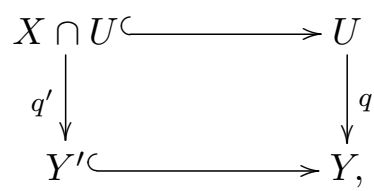

where $q^{\prime}$ is again a geometric quotient, and $Y^{\prime}$ is a Mori dream space. By applying the functor $\operatorname{Pic}^{*}(*)$, we can show that

$$
\operatorname{Pic}(Y)_{\mathbb{Q}} \stackrel{f}{\rightarrow} \operatorname{Pic}\left(Y^{\prime}\right)_{\mathbb{Q}} \rightarrow 0
$$

is surjective. Furthermore, by relating the Mori chamber structures through the GIT chamber structures of the prequotients, we can show that $f$ respects this chamber structure.

It seems very desirable to find torus-invariant hypersurfaces $X^{\prime}$ of $X$ which are again spectra of an AFD, because a positive answer in that direction would allow us to construct a flag of Mori dream spaces.

Of course, instead of considering the Cox ring construction of a Mori dream space, we could consider any other quotient of the spectrum $X$ of an AFD. Examples of such a situation are provided by representation varieties

$$
R_{d^{\prime}}\left(Q^{\prime}\right) \subset R_{d}(Q),
$$

where the quiver $Q^{\prime}$ is obtained from $Q$ by removing arrows or vertices.

\subsection{Application to quiver moduli}

From our point of view, quiver moduli form a class of examples for Mori dream spaces, which admits an at least partial description in a combinatorial flavor. An explicit instance of this philosophy is given in Examples 2.4.4 and 2.4.3. Conversely, the description of quiver moduli as Mori dream spaces might be helpful in understanding their birational geometry.

It has been observed before that Theorem 2.3 in [HK00] can be applied to some quiver moduli (see for example [Cr11]), but the assumption on the codimension of the unstable locus seems to restrict the possible applications.

For background on the construction of quiver moduli, consider Section 1.2. Recall that the representation variety with respect to a quiver $Q$ and dimension vector $d$ is defined as

$$
R_{d}(Q)=\bigoplus_{\alpha: i \rightarrow j} \operatorname{Hom}\left(k^{d_{i}}, k^{d_{j}}\right) .
$$


There is a canonical action of the reductive group

$$
P G_{d}=\left(\prod_{i \in Q_{0}} \mathrm{GL}_{d_{i}}(k)\right) / \mathbb{G}_{m}
$$

on $R_{d}(Q)$, given as simultaneous conjugation.

For a stability condition $\theta \in \mathbb{Z}^{Q_{0}}$, the moduli space $M_{d}^{\theta-\text { sst }}(Q)$ of quiver representations is then given as the GIT quotient of $R_{d}(Q)$ under $P G_{d}$ with respect to the character $\chi=\chi_{\theta}$.

Obviously, $P G_{d}$ is connected, and $R_{d}(Q)$ is just affine space. Note that if $d$ is coprime, which is that the greatest common divisor of the $d_{i}$ equals 1 , the set of stability conditions for which stability and semistability may differ is a finite union of hyperplanes (see Section 3.5 in [Rei08]). Thus for GIT chambers stability and semistability coincide. We thus have the following corollary.

THEOREM 2.3.1. Suppose that $Q$ is a quiver which does not admit oriented cycles, that $d$ is a coprime dimension vector, and that $\theta$ is a stability condition contained in the interior of a GIT chamber.

Then $\mathcal{M}_{d}^{\theta-\text { sst }}(Q)$ is a Mori dream space satisfying the assertions of Theorem 2.1.16. In particular, the Picard number is given as

$$
\rho\left(\mathcal{M}_{d}^{\theta-\text { sst }}(Q)\right)=\left|Q_{0}\right|-(r+1),
$$

where $r$ is the number of components of the unstable locus which are of codimension one.

If $Q$ admits oriented cycles, $\mathcal{M}_{d}^{\theta}(Q)$ is no longer projective, and hence fails to be a Mori dream space for trivial reasons. Since $\mathcal{M}_{d, \theta}(Q)$ is projective over the Hilbert quotient, the author expects that this however gives an example of a relative Mori dream space, which as of yet needs to be defined.

Furthermore, we note that the same results hold if we allow relations for the quiver, such that the associated representation variety is again a spectrum of an AFD. However, it seems very hard to describe relations which meet this criterion in general.

REMARK 2.3.2. There is an alternative proof showing that quiver moduli as in Theorem 2.3.1 are Mori dream spaces, which only makes use of the quotient result established in [HK00].

Consider the framed quiver $\widehat{Q}$ with additional stability parameter $\varepsilon$ attached to the additional point $\infty$ (we refer to [ER09], Section 3 for more details). We may take our framing to be large, in the sense that for each vertex $i \in Q_{0}$ we attach at least $n_{i}=d_{i}+1$ arrows $\infty \rightarrow i$.

Similarly to [ER09] Proposition 3.3, we see that for $\varepsilon \gg 0$ a representation $(M, f)$ of the framed quiver is stable if and only if it is semistable if and only if $f$ is dense, which is that there is no proper subrepresentation containing the vector space $k$ attached to $\infty$. Since the framing is large, we can always equip the arrows landing in each vertex $i \in Q_{0}$ with linear maps such that their images generate the vector space at $i$, and thus the unstable locus is 
contained in

$$
R_{d}(Q) \times \prod_{i \in Q_{0}} \operatorname{Hom}\left(k^{d_{i}+1}, k^{d_{i}}\right)_{\mathrm{rk} \leq d_{i}-1},
$$

which is well-known to be of codimension at least two. By Theorem 2.3 in [HK00], the associated quotient $\widehat{\mathcal{M}}_{\varepsilon \gg 0}$ is a Mori dream space, and so is the other chamber quotient $\widehat{\mathcal{M}}_{\varepsilon \approx 0}$, where we take $\varepsilon$ to be positive but sufficiently small ([HK00] Proposition 1.11.(2)). However, for this chamber there is the structure of a bundle

$$
\widehat{M}_{\varepsilon \approx 0} \rightarrow M
$$

where $\mathcal{M}$ is the moduli space for the unframed quiver ([ER09] Proposition 3.8). Hence $\mathcal{M}$ is a Mori dream space as well by the main result of [016]. However, it seems difficult to extract information about the Picard number or the Mori chambers using this construction.

Of course we could directly apply the result of [Ba11], since the prequotient $R_{d}(Q)$ is just affine space, but this does provide us with no quantitative information about the moduli space at all.

\subsection{Examples}

\subsubsection{Affine prequotients.}

We start with a very basic example, showing that Theorem 2.3 of [HK00] can be extended to the case of unstable components in codimension one.

EXAMPLE 2.4.1. Let the group $G=\mathbb{G}_{m} \times \mathbb{G}_{m}$ act on the variety

$$
X=\mathbb{A}^{1} \times \mathbb{A}^{2}
$$

via $(g, h) *(x, y)=(g x, h y)$, where we denote by $x$ the coordinate of $\mathbb{A}^{1}$ and by $y_{1}, y_{2}$ the coordinates on $\mathbb{A}^{2}$. It is easy to verify that there is only one GIT chamber with stable locus

$$
U=\left(\mathbb{A}^{1} \backslash 0\right) \times\left(\mathbb{A}^{2} \backslash 0\right),
$$

and associated quotient

$$
q: U \rightarrow \mathbb{P}^{0} \times \mathbb{P}^{1} \simeq \mathbb{P}^{1} .
$$

The quotient is a Mori dream space despite the fact that the unstable locus is of codimension one. However, the Picard number $\rho\left(\mathbb{P}^{1}\right)=1$ differs from the rank of the character group $\operatorname{rk}(\chi(G))=2$, as is predicted by Theorem 2.1.16. Indeed, we have that

$$
\mathcal{Z}(U)=\left\{0 \times \mathbb{A}^{2}\right\}
$$

consists of a single component. Furthermore, the relation between the stable GIT chambers and the Mori chambers is correctly predicted as well (though both are trivial). To see this note that

$$
0 \times \mathbb{A}^{2}=\mathrm{N}(x),(g, h) * x=g^{-1} x,
$$

so if we identify $\varphi: \mathbb{Z}^{2} \simeq \chi(G)$ via

$$
\varphi(a, b)=\left((g, h) \mapsto g^{-a} h^{-b}\right)
$$


the character associated to $0 \times \mathbb{A}^{2}$ is given as the vector $(-1,0)$ and the only GIT chamber is given as the cone spanned by the rays through the origin and $(-1,0)$ or $(0,-1)$ respectively.

In the first example we can easily replace $\mathbb{A}^{2}$ by any other prequotient.

EXAMPLE 2.4.2. Suppose that a reductive group $G$ acts on $X=\operatorname{Spec}(A)$, where $A$ is an AFD. Consider the action of the group $G^{\prime}=G \times \mathbb{G}_{m}$ on

$$
X^{\prime}=X \times \mathbb{A}^{1}
$$

given by $(g, h) *(x, y)=(g * x, h y)$. For the character lattice this corresponds to the addition of an orthogonal direction

$$
\chi\left(G^{\prime}\right) \simeq \chi(G) \times \mathbb{Z} .
$$

Since the action is diagonal, we can compute that

$$
\left(X^{\prime}\right)^{\chi-\text { sst }}=X^{\chi_{1}-\text { sst }} \times\left(\mathbb{A}^{1}\right)^{\chi_{2}-\text { sst }},
$$

where $\chi=\left(\chi_{1}, \chi_{2}\right)$ is the decomposition with respect to the above isomorphism. A similar statement holds for the stable loci. Hence the chamber structure for the action of $G$ on $X$ and for the action of $G^{\prime}$ on $X^{\prime}$ coincide in the sense that the chambers are stretched into the additional direction.

One can compute that for $U^{\prime}$ equal to the semistable locus to some chamber in $\chi\left(G^{\prime}\right)_{\mathbb{Q}}$ the unstable codimension one components are given as

$$
\mathcal{Z}\left(U^{\prime}\right)=\left\{Z \times \mathbb{A}^{1} \mid Z \in \mathcal{Z}(U)\right\} \cup\{X \times 0\},
$$

where $U$ is the semistable locus corresponding to the chamber in $\chi(G)_{\mathbb{Q}}$. The action of $G^{\prime}$ on $\mathcal{Z}\left(U^{\prime}\right)$ is induced by the action of $G$ on $\mathcal{Z}(U)$, where $X \times 0$ is fixed.

The associated characters for $Z \times \mathbb{A}^{1}$ lie in the hyperplane $\chi(G)_{\mathbb{Q}}$, and the character associated to $X \times 0$ points into the orthogonal direction. Thus, the stable chamber structure in $\chi\left(G^{\prime}\right)$ is induced by the stable chamber structure in $\chi(G)$, which agrees with the description of the quotients

$$
Y^{\prime} \simeq Y \times \mathbb{P}^{0} \simeq Y
$$

where $Y^{\prime}$ is the quotient with respect to a chamber in $\chi\left(G^{\prime}\right)_{\mathbb{Q}}$, and $Y$ is the quotient with respect to the corresponding chamber in $\chi(G)_{\mathbb{Q}}$.

Our first nontrivial example is the Hirzebruch surface $\mathcal{F}_{1}$, which also allows a description as a quiver moduli.

EXAmple 2.4.3. Consider the vector bundle $E=\mathcal{O}(0) \oplus \mathcal{O}(-1) \rightarrow \mathbb{P}^{1}$, and the associated projectivization

$$
\mathcal{F}_{1}=\mathbb{P}(\mathcal{O}(0) \oplus \mathcal{O}(-1)) \rightarrow \mathbb{P}^{1},
$$

which is the first Hirzebruch surface $\mathcal{F}_{1}$. Denoting by $\pi: \mathbb{A}^{2} \backslash 0 \rightarrow \mathbb{P}^{1}$ the canonical quotient map, we have the pullback $\pi^{*} E=L_{0} \oplus L_{-1} \rightarrow \mathbb{A}^{2} \backslash 0$, where as always $L \rightarrow \mathbb{A}^{2} \backslash 0$ is the trivial bundle, and 0 and -1 correspond to characters of $\mathbb{G}_{m}$ via the isomorphism $\chi\left(\mathbb{G}_{m}\right) \simeq \mathbb{Z}$. Note that as a variety $\pi^{*} E \simeq \mathbb{A}^{2} \times\left(\mathbb{A}^{2} \backslash 0\right)$, so taking out the image of the zero section gives the variety

$$
\left(\pi^{*} E\right)_{0} \simeq\left(\mathbb{A}^{2} \backslash 0\right) \times\left(\mathbb{A}^{2} \backslash 0\right) .
$$


There are two natural actions of $\mathbb{G}_{m}$ on $\left(\pi^{*} E\right)_{0}$. The first one is given by the description $\pi^{*} E=L_{0} \oplus L_{-1}$ and the second action is induced by the action of $\mathbb{G}_{m}$ on the fibres of $E$ (corresponding to the quotient $\pi^{\prime}: E_{0} \rightarrow \mathbb{P}(E)$ ). Since these two actions commute, we obtain an action of $G=\mathbb{G}_{m} \times \mathbb{G}_{m}$ on $\left(\pi^{*} E\right)_{0}$, which reads as

$$
(\lambda, \mu) *((a, b),(c, d))=((\lambda a, \lambda b),(\mu c, \lambda \mu d)) .
$$

We claim that the composition $\left(\pi^{*} E\right)_{0} \rightarrow E_{0} \rightarrow \mathbb{P}(E)=\mathcal{F}_{1}$ is a geometric quotient. Indeed, the construction of the involved bundles works locally over the open subsets where $E$ is trivial or the inverse images under $\pi$ thereof, and on these sets the claim holds.

We note that $\left(\pi^{*} E\right)_{0} \subset \mathbb{A}^{4}$ is the stable and semistable locus associated to the character $\chi(\lambda, \mu)=\lambda^{2} \mu$. Computing the GIT chambers for this action yields the following result, which is both accessible by an elementary calculation or by a quiver-like computation as in Example 2.4.4 (for the involved quiver see below).

\begin{tabular}{|c|c|c|}
\hline & stable locus & quotient \\
\hline \hline I & $(a, b) \neq 0 \wedge(c \neq 0 \vee d \neq 0)$ & $\mathcal{F}_{1}$ \\
\hline II & $c \neq 0 \wedge((a, b) \neq 0 \vee d \neq 0)$ & $\mathbb{P}^{2}$ \\
\hline
\end{tabular}

For chamber II, the formula for the Picard number as in Theorem 2.1.16 thus gives the correct result. We further remark that the only stable chamber with respect to chamber II is again chamber II, which agrees with the Mori chamber structure of $\mathbb{P}^{2}$.

Also, for chamber I, the theorem gives the correct result since there are no unstable codimension one components, and the GIT chamber structure agrees with the known Mori chamber structure of $\mathcal{F}_{1}$.

We can describe the morphisms induced by VGIT, or equivalently by the divisors, quite explicitly for this example. All of this agrees with the wellknown birational geometry of $\mathcal{F}_{1}$.

(1) For a character $\chi$ in the interior of chamber II, the map associated by VGIT

$$
f_{\chi}: \mathcal{F}_{1} \rightarrow \mathbb{P}^{2}
$$

is the blowup morphism, and is hence regular. This is also the contraction of the associated divisor $D=\psi(\chi)$, but $D$ is neither semiample nor movable.

(2) Further, for a character $\chi$ in the ray supporting both chamber I and the $G$-ample cone, the associated map

$$
f_{\chi}: \mathcal{F}_{1} \rightarrow \mathbb{P}^{1}
$$

is the map inducing the structure of $\mathcal{F}_{1}$ as a $\mathbb{P}^{1}$-bundle over $\mathbb{P}^{1}$.

(3) For a character in the ray supporting chamber II and the $G$-ample cone, the map induced by VGIT is trivial. 
Note that the action of $\mathbb{G}_{m} \times \mathbb{G}_{m}$ on $\mathbb{A}^{4}$ as above admits a description as the action of $P G_{d}$ on $R_{d}(Q)$, where the quiver $Q$ is given as

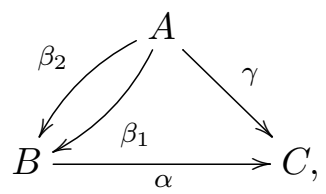

and the dimension vector as $d=(1,1,1)$ (compare with Example 3.6 in [CS08]).

\subsubsection{Quiver moduli examples.}

The following example provides a non-trivial stable chamber structure. Note that the group $G_{d}$ associated to the occurring dimension vector is a torus, and that the following quotients also admit a description in the toric language.

EXAMPLE 2.4.4. Let $Q$ denote the following quiver.

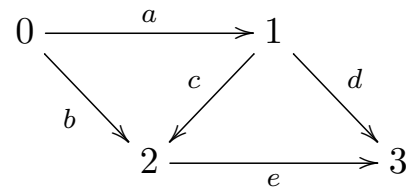

We consider the dimension vector $d=(1,1,1,1)$. If $M \in R_{d}(Q)$ is a representation, we use the notation $M=(a, b, c, d, e)$, where $a \in k$ is the linear map associated to the arrow $a$ and so forth.

As explained in section 2.3, the character group of $P G_{d}$ is identified with the hyperplane $(1,1,1,1)^{\perp} \subset \mathbb{Z}^{4} \simeq \chi\left(G_{d}\right)$. To facilitate the discussion, we will always extend the scalars to $\mathbb{R}$, and work with coordinates on $\chi\left(P G_{d}\right)_{\mathbb{R}}$ given by the basis

$$
\mathcal{B}: b_{1}=(1,1,-1,-1), b_{2}=(1,-1,1,-1), b_{3}=(1,-1,-1,1)
$$

for this hyperplane. To further simplify the picture, we consider the intersection of the GIT cone in $\mathbb{R}^{3}$ with an affine hyperplane $S$, given in parameter form as

$$
S: x(s, t)=p+s u_{1}+t u_{2},
$$

where $p=(1,1,0), u_{1}=(1,-1,0)$, and $u_{2}=(0,0,1)$. We can interpret this as the hyperplane of points which have value 1 in direction $(1,1,0)$. A GIT chamber in $\chi\left(P G_{d}\right)$ is thus represented by a polytope in the plane $\mathbb{R}^{2}$ corresponding to the coordinates on $S$.

We need to translate between cones, or half spaces, in $\chi\left(P G_{d}\right)$, and half spaces in $S$. This is a standard linear algebra computation, which gives the following result. Let $v \in(1,1,1,1)^{\perp} \subset \mathbb{R}^{4}$ denote a nonzero vector. Then the restriction of $v^{\perp} \subset \mathbb{R}^{4}$ to the slice $S$ in the coordinates as above is given as

$$
x(t)=a+t b,
$$


where

$$
b=\left(v_{1}+v_{4}, v_{3}-v_{2}\right),
$$

and $a$ is given by

$$
a=\left(0, \frac{v_{4}-v_{1}}{v_{1}+v_{4}}\right), \text { or } a=\left(\frac{v_{1}-v_{4}}{v_{3}-v_{2}}, 0\right) .
$$

If $v_{2}-v_{3}>0$, then the restriction of the half space $H(v) \subset \mathbb{R}^{4}$ with the slice $S$ is given by translating the line given above in the direction of the first coordinate. If $v_{2}-v_{3}<0$, the same holds with a change of direction. For the translation in the direction of the second coordinate a similar assertion holds when we use the inequalities $v_{1}+v_{4}>0$, or $v_{1}+v_{4}<0$.

We are now ready to compute the intersections of the 14 potential walls with $S$. Instead of a complete computation we focus on two prototypical examples and only state the full result without proof.

First, consider the dimension vector $e=(0,0,1,1)$. This dimension vector does always occur as the dimension type of a subrepresentation, and hence the associated hyperplane supports the $G$-ample cone. The condition for slope semistability reads as

$$
\frac{\theta_{2}+\theta_{3}}{2} \leq \frac{\theta_{0}+\theta_{1}+\theta_{2}+\theta_{3}}{4}
$$

Since this is equivalent to $0 \leq \theta_{0}+\theta_{1}-\theta_{2}-\theta_{3}$, the associated half space in $\chi\left(P G_{d}\right) \mathbb{R}$ is given as $H(1,1,-1,-1)$. A necessary condition for a character to be $G$-ample is thus that it is contained in the half space $H(1,1,-1,-1)$.

The dimension vector $e=(1,1,0,0)$ occurs as a subrepresentation of a representation $M=(a, b, c, d, e) \in R_{d}(Q)$ if and only if the maps starting in one of the points 0,1 and ending in of the points 2,3 are represented by 0 . That is, if and only if $b=c=d=0$.

Here, the condition for slope semistability reads as

$$
\frac{\theta_{0}+\theta_{1}}{2} \leq \frac{\theta_{0}+\theta_{1}+\theta_{2}+\theta_{3}}{4}
$$

which is equivalent to $0 \leq-\theta_{0}-\theta_{1}+\theta_{2}+\theta_{3}$. The associated half space in $\chi\left(P G_{d}\right)_{\mathbb{R}}$ is thus given as $H(-1,-1,1,1)$. For a character in $H(-1,-1,1,1)$ this dimension vector does not impose any condition on the semistable locus. However, if the character is not contained in the half space, a necessary condition for a representation to be semistable is that this dimension vector does not occur, which is $b \neq 0$ or $c \neq 0$ or $d \neq 0$.

Continuing the computation in the same spirit, the 14 potential dimension vectors of nontrivial subrepresentations give the following 14 potential walls. We also state into which direction the associated half space extends. 


\begin{tabular}{|c|c|c|c|c|c|}
\hline & $e$ & occurs iff & $a$ & $b$ & extends to \\
\hline \hline I & $(0,0,0,1)$ & (always) & $(0,2)$ & $(2,0)$ & neg. $y$-axis \\
\hline II & $(0,0,1,0)$ & $e=0$ & 0 & $(2,-4)$ & pos. $x$-axis \\
\hline III & $(0,1,0,0)$ & $c=d=0$ & 0 & $(2,4)$ & neg. $x$-axis \\
\hline IV & $(1,0,0,0)$ & $a=b=0$ & $(0,-2)$ & $(-2,0)$ & neg. $y$-axis \\
\hline V & $(1,1,0,0)$ & $b=c=d=0$ & $(-1,0)$ & $(0,2)$ & neg. $x$-axis \\
\hline VI & $(1,0,1,0)$ & $e=a=0$ & $(1,0)$ & $(0,-2)$ & pos. $x$-axis \\
\hline VII & $(1,0,0,1)$ & $a=b=0$ & 0 & $(-2,0)$ & neg. $y$-axis \\
\hline VIII & $(0,1,1,0)$ & $d=e=0$ & 0 & $(2,0)$ & pos. $y$-axis \\
\hline IX & $(0,1,0,1)$ & $c=0$ & $(1,0)$ & $(0,2)$ & neg. $x$-axis \\
\hline X & $(0,0,1,1)$ & $($ always $)$ & $(-1,0)$ & $(0,-2)$ & pos. $x$-axis \\
\hline XI & $(1,1,1,0)$ & $d=e=0$ & $(0,2)$ & $(2,0)$ & pos. $y$-axis \\
\hline XII & $(1,1,0,1)$ & $b=c=0$ & 0 & $(-2,4)$ & neg. $x$-axis \\
\hline XIII & $(1,0,1,1)$ & $a=0$ & 0 & $(-2,-4)$ & pos. $x$-axis \\
\hline XIV & $(0,1,1,1)$ & $($ always $)$ & $(0,-2)$ & $(2,0)$ & pos. $y$-axis \\
\hline
\end{tabular}

From this, we are able to read off the full chamber structure on $S$. The stability conditions for the chambers, which we sketch below in Figure 1, read as follows.

$$
\begin{aligned}
\left(R_{d}(Q)\right)^{A-\text { sst }}= & \{a \neq 0 \wedge e \neq 0 \wedge(b \neq 0 \vee c \neq 0 \vee d \neq 0)\} \\
\left(R_{d}(Q)\right)^{B-\text { sst }}= & \{a \neq 0 \wedge(b \neq 0 \vee c \neq 0) \wedge(d \neq 0 \vee e \neq 0)\} \\
\left(R_{d}(Q)\right)^{C-\text { sst }}= & \{(a \neq 0 \vee b \neq 0) \wedge(b \neq 0 \vee c \neq 0) \wedge(c \neq 0 \vee d \neq 0) \\
& \wedge(d \neq 0 \vee e \neq 0) \wedge(e \neq 0 \vee a \neq 0)\} \\
\left(R_{d}(Q)\right)^{D-\text { sst }}= & \{c \neq 0 \wedge(a \neq 0 \vee b \neq 0) \wedge(d \neq 0 \vee e \neq 0)\} \\
\left(R_{d}(Q)\right)^{E-\text { sst }}= & \{e \neq 0 \wedge(a \neq 0 \vee b \neq 0) \wedge(c \neq 0 \vee d \neq 0)\} .
\end{aligned}
$$

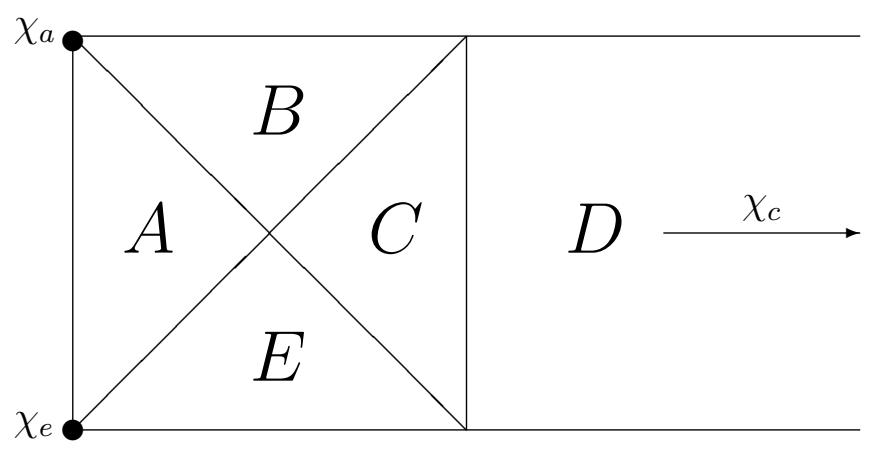

FiguRE 1. These are the restrictions of the GIT chambers to the slice $S$, where the $x$ - and $y$-axis correspond to coordinates on the slice and the $y$-axis is scaled with factor $\frac{1}{2}$. The origin is the point in which the chambers $A, B, C$ and $E$ meet. Additionally, the directions associated to possible unstable codimension one components are given. 
To compute which chambers are stable, we need to be able to translate directions in $\chi\left(P G_{d}\right)_{\mathbb{R}}$ to directions on the slice $S$. Hence suppose that we have a direction given as

$$
c=c_{p} p+c_{1} u_{1}+c_{2} u_{2} \in \mathbb{R}^{3},
$$

where $c_{p} \geq 0$, and an arbitrary vector

$$
x=p+s u_{1}+t u_{2} \in S .
$$

Then the translation of $x$ into the direction $c$ is given as

$$
x+n c=\left(1+n c_{p}\right) p+\left(s+n c_{1}\right) u_{1}+\left(t+n c_{2}\right) u_{2} .
$$

Obviously, the ray spanned by $x+n d$ through the origin meets the slice $S$ in the point

$$
p+\frac{s+n c_{1}}{1+n c_{p}} u_{1}+\frac{t+n c_{2}}{1+n c_{p}} u_{2} .
$$

If $c_{p} \neq 0$, this converges to the point $\left(c_{1}, c_{2}\right) \in S$ in the chosen coordinates on $S$. But if $c_{p}=0$, the translation into the direction of $c$ corresponds to the translation into the direction of $\left(c_{1}, c_{2}\right)$ in the coordinates on $S$.

Now we need the characters associated to codimension one components of the unstable loci. For the first coordinate function we have

$$
g * a=\frac{g_{0}}{g_{1}} a,
$$

which corresponds to the vector

$$
\chi_{a}=(1,-1,0,0) \in \chi\left(G_{d}\right)_{\mathbb{R}} .
$$

An elementary computation yields that this corresponds to a direction of the first type for $\chi_{a}=(-1,2)$ in coordinates on $S$.

With similar computation steps we obtain the directions $\chi_{e}=(-1,-2)$ of the first type, and $\chi_{c}=(1,0)$ of the second type.

This yields the following structure of stable chambers.

\begin{tabular}{|c|c|c|}
\hline chamber & $\mathcal{Z}$ & stable chambers \\
\hline \hline $\mathrm{A}$ & $\mathrm{N}(a), \mathrm{N}(e)$ & A \\
\hline $\mathrm{B}$ & $\mathrm{N}(a)$ & A,B \\
\hline $\mathrm{C}$ & $\emptyset$ & all chambers \\
\hline $\mathrm{D}$ & $\mathrm{N}(c)$ & $\mathrm{D}$ \\
\hline $\mathrm{E}$ & $\mathrm{N}(e)$ & $\mathrm{A}, \mathrm{E}$ \\
\hline
\end{tabular}

Finally, we compute some quotients associated to the chambers, showing that the Mori chamber structure of the quotients coincides with the stable GIT chamber structure. 
For the chamber B, we claim that the associated quotient is the Hirzebruch surface $\mathcal{F}_{1}$. Consider the maps

$$
\begin{aligned}
& \varphi: R_{d}(Q) \rightarrow R_{d^{\prime}}\left(Q^{\prime}\right),(a, b, c, d, e) \mapsto(e, b, c a, d a), \\
& \psi: R_{d^{\prime}}\left(Q^{\prime}\right) \rightarrow R_{d}(Q),\left(\alpha, \beta_{1}, \beta_{2}, \gamma\right) \mapsto\left(1, \beta_{1}, \beta_{2}, \gamma, \alpha\right),
\end{aligned}
$$

where the quiver $Q^{\prime}$ and the dimension vector $d^{\prime}$ are given as in Example 2.4.3. It is straightforward to check that $\varphi$ and $\psi$ respect the semistable loci of the chambers B and I respectively, and induce an isomorphism of the quotients.

Similarly the maps

$$
\begin{aligned}
& \varphi(a, b, c, d, e)=(e b, e c a, d a) \\
& \psi\left(\alpha_{1}, \alpha_{2}, \alpha_{3}\right)=\left(1, \alpha_{1}, \alpha_{2}, \alpha_{3}, 1\right),
\end{aligned}
$$

where $Q^{\prime}$ is given as

$$
A \rightleftharpoons B \text {, }
$$

show that the quotient with respect to the chamber $\mathrm{A}$ is isomorphic to $\mathbb{P}^{2}$.

For these chambers the predictions of Theorem 2.1.16 are thus confirmed. As a side remark we note that similar computations confirm the predictions for the chambers $\mathrm{D}$ and $\mathrm{E}$, where the quotients are both nontrivial $\mathbb{P}^{1}$-bundles over $\mathbb{P}^{1}$.

\subsubsection{Quotients of Mori dream spaces.}

We conclude with an example of a quotient of a Mori dream space.

EXAMPLE 2.4.5. The group $G=S L_{2}$ acts on the Mori dream space

$$
X=\left(\mathbb{P}^{1}\right)^{6}
$$

via the diagonalized canonical action on $\mathbb{P}^{1}$. Some quotients for this action are computed in [P95]. Following the notation given there, we consider quotients $X^{s}(m) / G$, where $m=\left(m_{1}, \ldots, m_{6}\right)$ indicates that we take the GIT quotient with respect to the bundle $\mathcal{O}\left(m_{1}\right) \otimes \ldots \otimes \mathcal{O}\left(m_{6}\right)$. Note that since the character group of $S L_{2}$ is trivial this bundle admits a unique $G^{-}$ linearization.

Corollary 5 in [P95] shows that in the case when $|m|=m_{1}+\ldots+m_{6}$ is odd, the corresponding bundle lies in the interior of a GIT chamber, and we can use Theorem 3 of [P95] to compute the unstable loci. 
Three geometric quotients are explicitly given. They are well-known to be Mori dream spaces (even toric), and the formula of Theorem 2.2.6 correctly predicts the Picard number.

(1) $X^{s}(2,3,3,1,1,1) / G \simeq B l_{(1,1,1),(\infty, \infty, \infty)}\left(\mathbb{P}^{1}\right)^{3}$, which has Picard number 5. Indeed, there is one unstable component of codimension one

$$
Z=\left\{\left(x_{1}, \ldots, x_{6}\right) \in\left(\mathbb{P}^{1}\right)^{6} \mid x_{2}=x_{3}\right\} .
$$

(2) $X^{s}(2,2,2,1,1,1) / G \simeq B l_{(0,0,0),(1,1,1),(\infty, \infty, \infty)}\left(\mathbb{P}^{1}\right)^{3}$, which has Picard number 6 . There are no unstable components of codimension one.

(3) $X^{s}(1,1,2,1,1,1) / G \simeq B l_{(0,0,0),(1,1,1),(\infty, \infty, \infty)}\left(\mathbb{P}^{1}\right)^{3}$, so the quotient again has Picard number 6 . In this case there are no unstable components of codimension one as well. 


\section{CHAPTER 3}

\section{Moduli spaces of quiver sheaves}

In this chapter, we provide a construction for the moduli spaces of multiGieseker semistable quiver sheaves, following Mumford's strategy. Furthermore, we discuss the variation of the moduli space under change of the stability condition.

\subsection{Quiver sheaves}

We start by introducing the notion of multi-Gieseker stability and the category of quiver sheaves. Also, we establish some foundational results about this notion of stability.

\subsubsection{Basic notions.}

Let $X$ denote any scheme, and let $Q$ denote an (unlabeled) quiver.

Definition 3.1.1. A quiver sheaf $\mathcal{E}$ on $X$ associated to the quiver $Q$ is a representation of $Q$ in the category of coherent sheaves on $X$. That is, we have a family of coherent sheaves $\mathcal{E}_{i}, i \in Q_{0}$ associated to the vertices, and a family $\mathcal{E}_{\alpha}: \mathcal{E}_{i} \rightarrow \mathcal{E}_{j}, \alpha: i \rightarrow j$ of morphisms of coherent sheaves associated to the arrows.

Occasionally, we will also consider representations of $Q$ in the category of quasi-coherent sheaves, and call them quasi-coherent quiver sheaves.

A morphism of quiver sheaves $\varphi: \mathcal{E} \rightarrow \mathcal{F}$ is a collection of morphisms $\varphi_{i}: \mathcal{E}_{i} \rightarrow \mathcal{F}_{i}$ for each vertex $i \in Q_{0}$, such that the canonical diagram

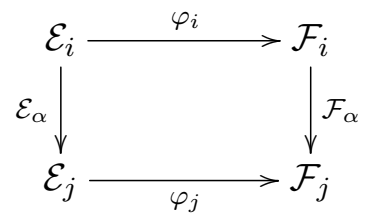

for each arrow $\alpha: i \rightarrow j$ commutes.

For a path $\gamma=\alpha_{l} \ldots \alpha_{1}$ and a quiver sheaf $\mathcal{E}$ recall that we denote

$$
\mathcal{E}_{\gamma}=\mathcal{E}_{\alpha_{l}} \ldots \mathcal{E}_{\alpha_{1}},
$$

and say that $\mathcal{E}$ satisfies a relation $\sum_{k=1}^{r} \lambda_{k} \gamma_{k}$ if the identity

$$
\sum_{k=1}^{r} \lambda_{k} \mathcal{E}_{\gamma_{k}}=0
$$


of morphisms of sheaves holds. This property is inherited by quiver subsheaves and quiver quotient sheaves.

To give a first idea about how to interpret quiver sheaves as configurations of morphisms of shevaes, we provide some simple examples.

EXAMPLE 3.1.2. The simplest non-trivial example of a quiver sheaf is a morphism

$$
\mathcal{E}_{1} \stackrel{\mathcal{E}_{\alpha}}{\longrightarrow} \mathcal{E}_{2},
$$

associated to the quiver consisting of two vertices and an arrow between them. Another example is that of a pair $(E, \phi)$, consisting of a sheaf $E$ and an endomorphism $\phi$. By using relations we could require

$$
\phi^{r}=0, \text { or } \phi^{r}=\phi,
$$

ie. that $\phi$ is nilpotent or idempotent respectively. A slightly more sophisticated example is that of two composable morphisms

$$
\mathcal{E}_{1} \stackrel{\mathcal{E}_{\alpha}}{\longrightarrow} \mathcal{E}_{2} \stackrel{\mathcal{E}_{\beta}}{\longrightarrow} \mathcal{E}_{3},
$$

where we could require $\mathcal{E}_{\beta} \mathcal{E}_{\alpha}=0$ by using relations. A commuting square of morphisms of sheaves can also be expressed as a quiver sheaf satisfying a relation.

Let us recall the notion of a topological type $\tau$ of a sheaf $E$ (compare with Definition 1.4 and Remark 1.5 of [GRT16]). It is given as

$$
\tau(E)=\operatorname{ch}(E) \operatorname{todd}(X) \in B(X)_{\mathbb{Q}},
$$

where $B(X)$ is the group of cycles on $X$ modulo algebraic equivalence (consider the proof of [F98] Theorem 15.2). By definition, the topological type thus remains constant in flat families over a connected and noetherian base scheme. Further, it determines the Hilbert polynomial with respect to any ample line bundle ([F98], Example 18.3.6).

By a slight abuse of notation we use the same letter for the topological type of a quiver sheaf.

DeFinition 3.1.3. We say that a quiver sheaf $\mathcal{E}$ has topological type

$$
\tau \in B(X)_{\mathbb{Q}}^{Q_{0}}
$$

if for all $i \in Q_{0}$ the sheaf $\mathcal{E}_{i}$ has topological type $\tau_{i}$.

There are some other properties of sheaves which transfer to quiver sheaves simply by requiring the sheaves at the vertices to fulfill this property.

Definition 3.1.4. A quiver sheaf $\mathcal{E}$ is called pure of dimension $d$ if all the shaves $\mathcal{E}_{i}$ are pure of dimension $d$.

Assume that $X$ is projective over $k$, and fix a tuple

$$
\underline{L}=\left(L_{1}, \ldots, L_{N}\right)
$$

of ample line bundles. Following [GRT16], we say that a sheaf $E$ is $(n, \underline{L})-$ regular if it is $n$-regular with respect to each line bundle $L_{j}$.

Definition 3.1.5. A quiver sheaf $\mathcal{E}$ is called $(n, \underline{L})$-regular if all the sheaves $\mathcal{E}_{i}$ are $(n, \underline{L})$-regular. Equivalently, the quiver sheaf $\mathcal{E}$ is $(n, \underline{L})$-regular if $\mathcal{E}_{i}$ is $n$-regular with respect to $L_{j}$ for all $i \in Q_{0}$ and all $j=1, \ldots, N$. 
Another such property is the saturation of sheaves.

Lemma 3.1.6. Let $\phi: E_{1} \rightarrow E_{2}$ denote a morphism of sheaves on $X$ which are of the same dimension $d$, and let $F_{1} \subset E_{1}, F_{2} \subset E_{2}$ denote subsheaves which are respected by the morphism, i.e. $\phi: F_{1} \rightarrow F_{2}$. Then the saturations

$$
\phi: F_{1, \mathrm{sat}} \rightarrow F_{2, \mathrm{sat}}
$$

are respected as well.

Proof. Recall ([HL10] Definition 1.1.5), that the saturation is given as

$$
F_{1, \text { sat }}=\operatorname{ker}\left(E_{1} \rightarrow E_{1} / F_{1} \rightarrow\left(E_{1} / F_{1}\right) /\left(T_{d-1}\left(E_{1} / F_{1}\right)\right)\right),
$$

where $T_{d-1}$ of a sheaf is defined to be the sum over all subsheaves of dimension at most $d-1$. We have an induced morphism $\phi^{\prime}: E_{1} / F_{1} \rightarrow E_{2} / F_{2}$. Since the dimension of a sheaf does not grow under taking the image, we have an induced morphism between the second quotients occurring in the above description of the saturation as well. The obvious commuting diagram tells us that $\phi$ thus induces a morphism between the kernels, which gives the result.

Lemma 3.1.6 shows that saturation of a quiver subsheaf is well-defined.

DEFINITION 3.1.7. Consider a quiver subsheaf $\mathcal{F} \subset \mathcal{E}$ of a quiver sheaf purely of dimension $d$. The saturation of $\mathcal{F}$ in $\mathcal{E}$ is defined as the quiver subsheaf $\mathcal{F}_{\text {sat }} \subset \mathcal{E}$ such that

$$
\left(\mathcal{F}_{\text {sat }}\right)_{i}=\mathcal{F}_{i, s a t}
$$

for all vertices $i \in Q_{0}$.

Definition 3.1.8. A family of quiver sheaves on $X$ is called flat or bounded over a $k$-scheme $S$ of finite type, if the families of sheaves $\mathcal{E}_{i}$ are flat or bounded for all $i \in Q_{0}$ respectively.

\subsubsection{Stability conditions.}

Now we introduce the notion of stability for quiver sheaves. To relate them to the already established notions of stability for vector space representations of a quiver ([K94]), and to the stability condition for sheaves as in [GRT16], we first give a slightly more general definition which interpolates between these two. However, our construction and variation results only hold in a special case.

Consider a projective scheme $X$ over $k$.

Definition 3.1.9. A multi-Gieseker stability condition $(\underline{L}, \sigma, \rho)$ for quiver sheaves associated to $Q$ over $X$ consists of a tuple

$$
\underline{L}=\left(L_{1}, \ldots, L_{N}\right)
$$

of ample line bundles on $X$, and two tuples

$$
\sigma, \rho \in\left(\mathbb{R}^{Q_{0} \times N}\right)_{+},
$$


where the right hand side is the subset of $\mathbb{R}^{Q_{0} \times N}$ consisting of tuples $\sigma$ of non-negative real numbers such that for any fixed $i$ not all $\sigma_{i j}$ vanish simultaneously. We denote the entries of such tuples $\sigma$ as

$$
\sigma=\left(\sigma_{i j}\right)_{(i, j) \in Q_{0} \times N} \text {. }
$$

If all entries of $\sigma$ are strictly positive we say that $\sigma$ is positive.

Typically, we think of $\underline{L}$ as being fixed, and just refer to $(\sigma, \rho)$ as the stability condition if $\underline{L}$ is clear from the context.

Definition 3.1.10. The multi-Hilbert polynomial of a quiver sheaf $\mathcal{E}$ with respect to $\sigma$ is defined as

$$
P_{\mathcal{E}}^{\sigma}=\sum_{i \in Q_{0}} \sum_{j=1}^{N} \sigma_{i j} P_{\mathcal{E}_{i}}^{L_{j}},
$$

where $P_{\mathcal{E}_{i}}^{L_{j}}$ is the usual Hilbert polynomial of $\mathcal{E}_{i}$ computed with respect to the ample line bundle $L_{j}$.

The multi-Hilbert polynomial can be written as

$$
P_{\mathcal{E}}^{\sigma}(T)=\frac{\alpha_{d}^{\sigma}(\mathcal{E})}{d !} T^{d}+\frac{\alpha_{d-1}^{\sigma}(E)}{(d-1) !} T^{d-1}+\ldots+\alpha_{0}^{\sigma}(\mathcal{E})
$$

for real numbers $\alpha_{k}^{\sigma}(\mathcal{E})$, and strictly positive leading coefficient. Here, $d$ is the maximum of the dimensions of the sheaves $\mathcal{E}_{i}$. The coefficients can be further expressed as

$$
\alpha_{k}^{\sigma}(\mathcal{E})=\sum_{i \in Q_{0}} \sum_{j=1}^{N} \sigma_{i j} \alpha_{k}^{L_{j}}\left(\mathcal{E}_{i}\right),
$$

where $\alpha_{k}^{L_{j}}\left(\mathcal{E}_{i}\right) / k$ ! is the coefficient to the monomial $T^{k}$ in the Hilbert polynomial $P_{\mathcal{E}_{i}}^{L_{j}}$, computed with respect to $L_{j}$.

Using these coefficients allows us to introduce the reduced version of the multi-Hilbert polynomial and, later, the slope.

Definition 3.1.11. The multi-rank of a quiver sheaf $\mathcal{E}$ with respect to $\rho$ is defined as

$$
\operatorname{rk}^{\rho}(\mathcal{E})=\sum_{i \in Q_{0}} \sum_{j=1}^{N} \rho_{i j} \alpha_{d}^{L_{j}}\left(\mathcal{E}_{i}\right),
$$

where $d$ is the maximum of the dimensions of the sheaves $\mathcal{E}_{i}$. The reduced multi-Hilbert polynomial of $\mathcal{E}$ with respect to $(\sigma, \rho)$ is defined as

$$
p_{\mathcal{E}}^{(\sigma, \rho)}=\frac{P_{\mathcal{E}}^{\sigma}}{\mathrm{rk}^{\rho}(\mathcal{E})} .
$$

Definition 3.1.12. A quiver sheaf $\mathcal{E}$ is called multi-Gieseker semistable with respect to $(\sigma, \rho)$, or simply semistable, if it is pure and for all nontrivial quiver subsheaves $\mathcal{F} \subset \mathcal{E}$ the inequality

$$
p_{\mathcal{F}}^{(\sigma, \rho)} \leq p_{\mathcal{E}}^{(\sigma, \rho)}
$$

holds. If all such inequalities are strict we call $\mathcal{E}$ stable. 
REMARK 3.1.13. This stability condition is a condition in the sense of [R97]. Additionally, any quiver sheaf $\mathcal{E}$ is noetherian, allowing us to adopt general results from $[\mathrm{R} 97]$ to our setting.

There are three special cases of this stability condition.

(1) In case $X=\operatorname{Spec}(k)$, coherent sheaves are just vector spaces $V$, and the Hilbert polynomial is just its dimension. Consequently, quiver sheaves are simply vector space representations $M$ of $Q$. Considering $\underline{L}=(L)$, i.e. just the trivial line bundle, $\sigma=\theta$ and $\rho=(1,1, \ldots, 1)$, we compute

$$
p^{(\sigma, \rho)}(M)=\frac{\sum_{i \in Q_{0}} \theta_{i} \operatorname{dim}\left(M_{i}\right)}{\sum_{i \in Q_{0}} \operatorname{dim}\left(M_{i}\right)} .
$$

In other words, $(\sigma, \rho)$-stability is the same as the well-known slope stability for vector space representations of $Q$ as introduced in [K94].

(2) If $Q=\bullet$ is the trivial quiver, quiver sheaves are the same as sheaves $E$ on $X$. Considering $\sigma=\rho$, the reduced multi-Hilbert polynomial for a $d$-dimensional sheaf $E$ reads as

$$
p_{E}^{(\sigma, \sigma)}=\frac{\sum_{j=1}^{N} \sigma_{j} P_{E}^{L_{j}}}{\sum_{j=1}^{N} \sigma_{j} \alpha_{d}^{L_{j}}(E)} .
$$

Thus this stability condition is the one introduced by [GRT16].

(3) From now on we consider the case of an arbitrary scheme $X$, an arbitrary quiver $Q$, but $\sigma=\rho$. The reduced multi-Hilbert polynomial of a quiver sheaf $\mathcal{E}$ then reads as

$$
p_{\mathcal{E}}^{\sigma}=\frac{\sum_{i \in Q_{0}} \sum_{j=1}^{N} \sigma_{i j} P_{\mathcal{E}_{i}}^{L_{j}}}{\sum_{i \in Q_{0}} \sum_{j=1}^{N} \sigma_{i j} \alpha_{d}^{\sigma}\left(\mathcal{E}_{i}\right)} .
$$

Of course it is convenient to just write $\sigma$ instead of $(\sigma, \sigma)$ in this case. Sometimes we need to compare our stability condition to stability conditions for the sheaves at the vertices in the sense of [GRT16].

DEFINITION 3.1.14. Let $(\underline{L}, \sigma)$ denote a stability condition for quiver sheaves on $X$ associated to a quiver $Q$. We use the notation

$$
\sigma_{i_{0}}=\left(\sigma_{i_{0} j}\right)_{j=1, \ldots, N}
$$

for the restriction of $\sigma$ to the vertex $i_{0} \in Q_{0}$. The tuple $\left(\underline{L}, \sigma_{i_{0}}\right)$ then is a stability condition for sheaves on $X$ in the sense of [GRT16].

\subsubsection{Properties of stability.}

From now on we restrict ourselves to the special case of $\rho=\sigma$, and examine some of the properties of such conditions. In this case, the leading coefficient of the reduced multi-Hilbert polynomial is $1 / d$ !, and thus not very interesting. The second highest order coefficient is of more importance.

Definition 3.1.15. The slope of a quiver sheaf $\mathcal{E}$ with respect to a stability condition $\sigma$ is

$$
\widehat{\mu}^{\sigma}(\mathcal{E})=\frac{\alpha_{d-1}^{\sigma}(\mathcal{E})}{\alpha_{d}^{\sigma}(\mathcal{E})},
$$

where $d$ is the degree of the multi-Hilbert polynomial. 
We need the following easy technical result.

Lemma 3.1.16. Let $\mathcal{E}$ denote a quiver sheaf such that

$$
\widehat{\mu}^{\sigma}(\mathcal{E}) \geq \mu
$$

for some real number $\mu$. Then there exist indices $1 \leq j \leq N$ and $i \in Q_{0}$ such that $\sigma_{i j} \neq 0$ and $\widehat{\mu}^{L_{j}}\left(\mathcal{E}_{i}\right) \geq \mu$.

Proof. With the obvious modifications the elementary proof of [GRT16] Lemma 2.11 applies.

The reduced multi-Hilbert polynomial can now be written as

$$
p_{\mathcal{E}}^{\sigma}(T)=\frac{1}{d !} T^{d}+\frac{\widehat{\mu}^{\sigma}(\mathcal{E})}{(d-1) !} T^{d-1}+\mathcal{O}\left(T^{d-2}\right) .
$$

As an instance of the philosophy mentioned in Remark 3.1.13, there are variants of the Harder-Narasimhan and Jordan-Hölder filtration for quiver sheaves. Alternatively, one could check that the proofs of the respective results for sheaves (eg. consider Proposition 1.5.2 and Theorem 1.3.4 in [HL10]) can be generalized.

The Harder-Narasimhan filtration measures to what extent a quiver sheaf fails to be semistable.

THEOREM 3.1.17. For a pure quiver sheaf $\mathcal{E}$ and a stability condition $\sigma$ there is a uniquely determined filtration

$$
0=\mathrm{HN}^{0}(\mathcal{E}) \subsetneq \mathrm{HN}^{1}(\mathcal{E}) \subsetneq \ldots \subsetneq \mathrm{HN}^{l}(\mathcal{E})=\mathcal{E},
$$

called the Harder-Narasimhan filtration of $\mathcal{E}$, such that all the subquotients $\mathcal{F}_{k+1}=\mathrm{HN}^{k+1}(\mathcal{E}) / \mathrm{HN}^{k}(\mathcal{E})$ are semistable and such that

$$
p^{\sigma}\left(\mathcal{F}^{1}\right)>p^{\sigma}\left(\mathcal{F}^{2}\right)>\ldots>p^{\sigma}\left(\mathcal{F}^{l}\right) .
$$

Further, $p_{\max }^{\sigma}(\mathcal{E})=p^{\sigma}\left(\mathrm{HN}^{1}(\mathcal{E})\right)$ is maximal among the reduced Hilbert polynomials of quiver subsheaves of $\mathcal{E}$, and $\mathrm{HN}^{1}(\mathcal{E})$ is maximal among the quiver subsheaves attaining this limit.

If a quiver sheaf is semistable, the Jordan-Hölder filtration describes if and how it fails to be stable.

TheOREM 3.1.18. Suppose that $\mathcal{E}$ is a semistable quiver sheaf with respect to a stability condition $\sigma$. There exists a filtration

$$
0=\mathcal{E}^{0} \subsetneq \mathcal{E}^{1} \subsetneq \ldots \subsetneq \mathcal{E}^{l}=\mathcal{E},
$$

called a Jordan-Hölder filtration, such that all $\mathcal{E}^{k}$ have the same reduced multi-Hilbert polynomial and such that all subquotients are stable.

The subquotients in any such filtration are uniquely determined up to isomorphism and permutation.

The uniqueness of the subquotients in a Jordan-Hölder filtration implies that the following notion is well-defined.

Definition 3.1.19. Fix a stability condition $\sigma$ and consider two semistable quiver sheaves $\mathcal{E}$ and $\mathcal{F}$. We define

$$
\operatorname{gr}(\mathcal{E})=\bigoplus_{k=1}^{l} \mathcal{E}^{k} / \mathcal{E}^{k-1},
$$


where the $\mathcal{E}^{k}$ are the quiver subsheaves occurring in some Jordan-Hölder filtration of $\mathcal{E}$. We say that $\mathcal{E}$ and $\mathcal{F}$ are $S$-equivalent if

$$
\operatorname{gr}(\mathcal{E}) \simeq \operatorname{gr}(\mathcal{F})
$$

As in the case of sheaves, it is enough to check the inequalities on saturated quiver subsheaves.

Lemma 3.1.20. A pure quiver sheaf $\mathcal{E}$ on $X$ is semistable with respect to a stability condition $\sigma$ if and only if

$$
p_{\mathcal{F}}^{\sigma} \leq p_{\mathcal{E}}^{\sigma}
$$

holds for all nontrivial saturated quiver subsheaves $\mathcal{F} \subset \mathcal{E}$. Similarly, $\mathcal{E}$ is stable if strict inequality holds for all such quiver subsheaves.

Proof. Consider any quiver subsheaf $\mathcal{F} \subset \mathcal{E}$. By construction, the saturation of $\mathcal{F}$ differs from $\mathcal{F}$ only in codimension one, and is a supersheaf of $\mathcal{F}$. Hence

$$
\alpha_{d}^{L_{j}}\left(\mathcal{F}_{i, \text { sat }}\right)=\alpha_{d}^{L_{j}}\left(\mathcal{F}_{i}\right) \text { and } P_{\mathcal{F}_{i}}^{L_{j}} \leq P_{\mathcal{F}_{i, s a t}}^{L_{j}}
$$

for all $i \in Q_{0}$ and $j=1, \ldots, N$, where $d$ is the dimension of $\mathcal{E}$. Thus the reduced multi-Hilbert polynomial of $\mathcal{F}$ is always smaller than the polynomial of the saturation.

We can prove a generalized version of a lemma of Grothendieck, which is a central boundedness result in our work. To prepare for the variation of moduli spaces as treated in Section 3.6, we formulate this result for a whole set of stability conditions. Consider a subset

$$
\Sigma \subset\left(\mathbb{R}^{Q_{0} \times N}\right)_{+}
$$

which is a closed cone without origin in $\mathbb{R}^{Q_{0} \times N}$. Clearly, we could also consider subsets which are contained in such a closed cone, or simply a single parameter $\sigma$.

LEMma 3.1.21. Fix a stability condition $\sigma$, integers $p$ and $d$, a real number $\mu$, a topological type $\tau$, and a cone $\Sigma$ as given above. Consider the family $S$ of quiver sheaves $\mathcal{F}$ which arise as quiver subsheaves of some quiver sheaf $\mathcal{E}$ such that

(1) $\mathcal{E}$ is $(p, \underline{L})$-regular of topological type $\tau$,

(2) $\mathcal{F}$ is saturated in $\mathcal{E}$,

(3) $\mathcal{E}$ is pure of dimension $d$,

(4) and $\widehat{\mu}^{\sigma}(\mathcal{F}) \geq \mu$ for some $\sigma \in \Sigma$.

This family is bounded.

Proof. We proceed by induction on $\left|Q_{0}\right|$.

In the case $\left|Q_{0}\right|=1$ the quiver subsheaves of some quiver sheaf $\mathcal{E}$ are clearly a subfamily of the family of subsheaves, and the boundedness of this family is settled by [GRT16], Lemma 4.5, invoking a lemma of Grothendieck ([HL10] Lemma 1.7.9). Note that this proof also works in the case of pure sheaves of arbitrary dimension.

In the general case, we get by Lemma 3.1.16 that $S$ decomposes into a finite union of families $S_{i j}$ where at least one sheaf satisfies $\widehat{\mu}^{L_{j}}\left(\mathcal{F}_{i}\right) \geq \mu$. Thus by the lemma of Grothendieck the family of sheaves $\mathcal{F}_{i}$ occurring at the vertex $i$ 
in quiver sheaves contained in $S_{i j}$ is bounded (note that we can equivalently pass to the quotient sheaves since $\mathcal{F}_{i}$ is saturated in $\mathcal{E}_{i}$ ). It suffices to prove that the families of the $\mathcal{F}_{i^{\prime}}, i \neq i^{\prime}$, occurring in $S_{i j}$, are bounded as well.

We concentrate on the case of $S_{1 j}$. In particular, the values of $\alpha_{e}^{L_{j}}\left(\mathcal{F}_{1}\right)$ are confined to a finite set, and for each of value in this set, the expression

$$
\widehat{\mu}^{\sigma_{1}}\left(\mathcal{F}_{1}\right)=\frac{\alpha_{d-1}^{\sigma_{1}}\left(\mathcal{F}_{1}\right)}{\alpha_{d}^{\sigma_{1}}\left(\mathcal{F}_{1}\right)}
$$

defines a well-defined and continuous function in $\sigma \in \Sigma$. Furthermore, this function is invariant under simultaneous scaling, and must thus be bounded; note that, modulo scaling, $\Sigma$ yields a compact set. Hence,

$$
\mu^{\prime} \geq \widehat{\mu}^{\sigma_{1}}\left(\mathcal{F}_{1}\right)
$$

uniformly in $\mathcal{F}_{1}$ and $\sigma$ for some real number $\mu^{\prime}$. Note that by the dimension assumption the $\alpha_{d}^{\sigma_{i}}\left(\mathcal{F}_{i}\right)$ are non-negative, so that we have

$$
\begin{aligned}
\frac{\sum_{1 \neq i \in Q_{0}} \alpha_{d-1}^{\sigma_{i}}\left(\mathcal{F}_{i}\right)}{\sum_{1 \neq i \in Q_{0}} \alpha_{d}^{\sigma_{i}}\left(\mathcal{F}_{i}\right)} & \geq \frac{\mu\left(\sum_{i \in Q_{0}} \alpha_{d}^{\sigma_{i}}\left(\mathcal{F}_{i}\right)\right)-\alpha_{d-1}^{\sigma_{1}}\left(\mathcal{F}_{1}\right)}{\sum_{1 \neq i \in Q_{0}} \alpha_{d}^{\sigma_{i}}\left(\mathcal{F}_{i}\right)} \\
& \geq \frac{\mu\left(\sum_{1 \neq i \in Q_{0}} \alpha_{d}^{\sigma_{i}}\left(\mathcal{F}_{i}\right)\right)+\left(\mu-\mu^{\prime}\right) \alpha_{d}^{\sigma_{1}}\left(\mathcal{F}_{1}\right)}{\sum_{1 \neq i \in Q_{0}} \alpha_{d}^{\sigma_{i}}\left(\mathcal{F}_{i}\right)} \\
& =\mu+\left(\mu-\mu^{\prime}\right) \frac{\alpha_{d}^{\sigma_{1}}\left(\mathcal{F}_{1}\right)}{\sum_{1 \neq i \in Q_{0}} \alpha_{d}^{\sigma_{i}}\left(\mathcal{F}_{i}\right)}
\end{aligned}
$$

The left hand side is the slope of the restriction of $\mathcal{F}$ to the quiver where the vertex 1 , together with all arrows starting and ending in it, are removed. We claim that the right hand side is bounded from below, which would finish the proof by induction. Indeed, the fraction on the right hand side can be estimated as

$$
\begin{aligned}
\frac{\alpha_{d}^{\sigma_{1}}\left(\mathcal{F}_{1}\right)}{\sum_{1 \neq i \in Q_{0}} \alpha_{d}^{\sigma_{i}}\left(\mathcal{F}_{i}\right)} & =\frac{\alpha_{d}^{\sigma_{1}}\left(\mathcal{F}_{1}\right)}{\sum_{i \in I} \sum_{j=1}^{N} \sigma_{i j} \alpha_{d}^{L_{j}}\left(\mathcal{F}_{i}\right)} \\
& \leq \frac{\alpha_{d}^{\sigma_{1}}\left(\mathcal{F}_{1}\right)}{\sum_{i \in I} \sum_{j=1}^{N} \sigma_{i j}}
\end{aligned}
$$

where $I \subset Q_{0}$ is the subset of vertices $i \in Q_{0}$ not equal to 1 for which $\mathcal{F}_{i}$ does not vanish. For these vertices, $\alpha_{d}^{L_{j}}\left(\mathcal{F}_{i}\right)$ is integral and non-vanishing, giving us the inequality. Now the right hand side is also a well-defined and continuous function which is invariant under scaling, and is hence bounded.

REMARK 3.1.22. The choice of a closed cone $\Sigma$ is to obtain a compact set after modding out scaling. For the final inequality in the proof of Lemma 3.1.21 to work, we also need that $\Sigma$ does not meet the boundary. 
To talk about stability in families we consider

$$
\underline{L}^{\prime}=\left(L_{1}^{\prime}, \ldots, L_{N}^{\prime}\right),
$$

the pullback of $\underline{L}$ to the space $X \times S$ where the family lives on. Note that $\underline{L}^{\prime}$ consists of relatively ample line bundles.

Proposition 3.1.23. Being pure, semistability and stability are open properties for flat families of quiver sheaves.

Proof. Let $\mathcal{E}$ denote a flat family of quiver sheaves of topological type $\tau$ on $X$ over some noetherian base scheme $S$, and consider the family of relatively ample line bundles $\underline{L}^{\prime}$ as in the preceding remark.

We know by definition that $\mathcal{E}_{s}$, where $s \in S$ is a geometric point, is pure if and only if all the sheaves $\mathcal{E}_{s, i}$ for $i \in Q_{0}$ are pure. Thus openness is implied by the openness of the sheaf version as provided by [HL10] Proposition 2.3.1.

To prove openness of stability and semistability we also follow the reasoning of [HL10]. For the sake of this proof we introduce the notation $\widehat{\mu}^{\sigma}(\tau)$ and $p^{\sigma}(\tau)$ for the slope and reduced Hilbert polynomial fixed by the topological type and the stability condition. Consider the set $A \subset B(X)_{\mathbb{Q}}^{Q_{0}}$ of topological types $\tau^{\prime}$ such that $\widehat{\mu}^{\sigma}\left(\tau^{\prime}\right) \leq \widehat{\mu}^{\sigma}(\tau)$ and such that there exists a geometric point $s \in S$ and a pure quotient $\mathcal{E}_{s} \rightarrow \mathcal{E}^{\prime}$ with the property $\tau\left(\mathcal{E}^{\prime}\right)=\tau^{\prime}$ and $p^{\sigma}\left(\tau^{\prime}\right)<p^{\sigma}(\tau)$. By the Grothendieck Lemma for quiver sheaves 3.1.21, the family of quotient quiver sheaves $\mathcal{E}^{\prime}$ underlying $A$ is bounded, so in particular $A$ is finite.

Note that $\mathcal{E}_{s}$ is semistable if and only if $s$ is contained in the complement of the union of the finitely many closed images of the morphisms

$$
\operatorname{Quot}_{\mathcal{E} / X / S}^{\tau^{\prime}} \rightarrow S
$$

where $\tau^{\prime} \in A$. Here we use the Quiver Quot-scheme as introduced in Section 3.8. For stability, we use a similar argument using the inequality $p^{\sigma}\left(\tau^{\prime}\right) \leq$ $p^{\sigma}(\tau)$ instead of strict inequality in the definition of $A$.

A necessary condition for the construction of the moduli space is the boundedness of the family of semistable quiver sheaves. In contrast to the case of Gieseker-semistable sheaves this is not automatic. For a partial result on this problem, consider Theorem 3.7.7. Again, we think of $\underline{L}$ and also of $\tau$ as fixed. Additionally, we fix a (possibly empty) set $I$ of relations on $Q$.

Definition 3.1.24. A subset

$$
\Sigma \subset\left(\mathbb{R}^{Q_{0} \times N}\right)_{+}
$$

is called a bounded set of stability conditions if the family of quiver sheaves $\mathcal{E}$, which are of topological type $\tau$, satisfy the relations $I$, and $\sigma$-semistable for some $\sigma \in \Sigma$, is bounded. In case that $\Sigma$ contains a single element $\sigma$, we say that $\sigma$ is bounded.

REMARK 3.1.25. Clearly, imposing more relations can only improve the boundedness of some set $\Sigma$.

Suppose we have a fixed projective scheme $X$, a stability condition $(\underline{L}, \sigma)$, a topological type $\tau$ and a set of relations $I$ on a quiver $Q$. 
Definition 3.1.26. The moduli functor of semistable quiver sheaves

$$
\mathcal{M}^{\text {sst }}=\mathcal{M}_{\tau}^{\sigma-\text { sst }}(Q, I, X):(S c h / k)^{\text {op }} \rightarrow \text { Sets }
$$

sends a scheme $S$ to the set of isomorphism classes of families which are flat over $S$ and consist of quiver sheaves on $X$ of topological type $\tau$ which are $\sigma$-semistable and satisfy the relations $I$. There is a similar notion of a moduli functor

$$
\mathcal{M}^{\text {st }}=\mathcal{M}_{\tau}^{\sigma-\mathrm{st}}(Q, I, X)
$$

for stable quiver sheaves.

Following [Si94], Section 1, we introduce the notion of moduli spaces.

Definition 3.1.27. A scheme $M^{\text {sst }}=M_{\tau}^{\sigma-\text { sst }}(Q, I, X)$ which corepresents $\mathcal{M}^{\text {sst }}$ is called the (coarse) moduli space of semistable quiver sheaves of topological type $\tau$. Similarly, the moduli space $M^{\text {st }}=M_{\tau}^{\sigma-s t}(Q, I, X)$ of stable quiver sheaves is required to corepresent $\mathcal{M}^{\text {st }}$.

If $M^{\text {sst }}$ or $M^{\text {st }}$ represent the respective moduli functor, they are called fine moduli spaces.

\subsection{The embedding functor}

According to our program of construction, we need a functor that embeds the category of sheaves into the category of representations of a quiver $Q\left(Q^{\prime}\right)$, which we need to construct.

\subsubsection{The twisted quiver.}

Our embedding functor maps quiver sheaves to representations of the twisted quiver $Q\left(Q^{\prime}\right)$, where the twisting, or auxiliar, quiver is given as in [GRT16].

Fix a projective scheme $X$ and a collection $\underline{L}=\left(L_{1}, \ldots, L_{N}\right)$ of ample line bundles on $X$. Furthermore, we fix a topological type $\tau$ of a quiver sheaf and two natural numbers $m>n$.

Definition 3.2.1. The auxiliary quiver with $N$ rows is defined as follows.

$$
\begin{aligned}
& Q_{0}^{\prime}=\left\{v_{1}, \ldots, v_{N}, w_{1}, \ldots, w_{N}\right\} \\
& Q_{1}^{\prime}=\left\{\varphi_{k l}: v_{k} \rightarrow w_{l} \mid k, l=1, \ldots, N\right\} .
\end{aligned}
$$

The labels $H_{i j}=H_{\varphi_{i j}}$, dependent on $\underline{L}, m$ and $n$, attached to the arrows $\varphi_{i j}: v_{i} \rightarrow w_{j}$ are given as

$$
H_{i j}=H^{0}\left(X, L_{i}^{-n} \otimes L_{j}^{m}\right)=\operatorname{Hom}\left(L_{i}^{n}, L_{j}^{m}\right) .
$$

For example, the auxiliary quiver $Q^{\prime}$ for $N=3$ looks as follows (ignoring the labels).

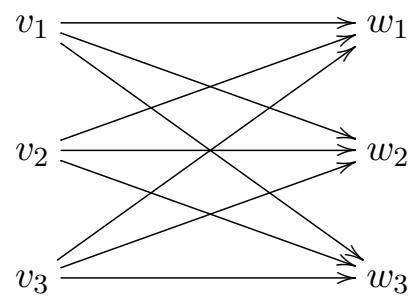


For integers $m>n$ consider the sheaf

$$
T=\bigoplus_{j=1}^{N} L_{j}^{-n} \oplus L_{j}^{-m}
$$

and the $k$-algebra

$$
A^{\prime}=L \oplus H \subset \operatorname{End}_{X}(T),
$$

where $L$ is generated by the projections onto the summands $L_{i}^{-n}$ and $L_{j}^{-m}$ and

$$
H=\bigoplus_{i, j=1}^{N} H_{i j}, H_{i j}=H^{0}\left(X, L_{i}^{-n} \otimes L_{j}^{m}\right)=\operatorname{Hom}_{X}\left(L_{j}^{-m}, L_{i}^{-n}\right) .
$$

The algebra $A^{\prime}$ is realized as the path algebra of the auxiliary quiver $Q^{\prime}$ with $N$ rows and labels $H_{i j}$.

Definition 3.2.2. Let $Q$ denote any finite quiver, and consider the auxiliary quiver $Q^{\prime}$ for some given $N, m$ and $n$ with given labels $H_{i j}$. Then the twisted quiver $Q\left(Q^{\prime}\right)$ is defined as follows.

$$
\begin{aligned}
Q\left(Q^{\prime}\right)_{0} & =\left\{v_{i j}, w_{i j} \mid i \in Q_{0}, j=1, \ldots, N\right\} \\
Q\left(Q^{\prime}\right)_{1} & =\left\{\varphi_{i k l}: v_{i k} \rightarrow w_{i l} \mid i \in Q_{0}, k, l=1, \ldots, N\right\} \\
& \cup\left\{\alpha_{k}^{\text {left }}: v_{i k} \rightarrow v_{j k} \mid(\alpha: i \rightarrow j) \in Q_{1}, k=1, \ldots, N\right\} \\
& \cup\left\{\alpha_{k}^{\text {right }}: w_{i k} \rightarrow w_{j k} \mid(\alpha: i \rightarrow j) \in Q_{1}, k=1, \ldots, N\right\}
\end{aligned}
$$

The label attached to an arrow $\varphi_{i k l}$ is $H_{k l}=H^{0}\left(L_{k}^{-n} \otimes L_{l}^{m}\right)$, independent of $i \in Q_{0}$, and the arrows $\alpha_{k}^{\text {left }}$ and $\alpha_{k}^{\text {right }}$ remain unlabeled. Further, for any path $\gamma=\alpha_{l} \ldots \alpha_{1}$ in $Q$ and any integer $1 \leq k \leq N$ we define

$$
\gamma_{k}^{\text {right }}=\left(\alpha_{l}\right)_{k}^{\text {right }} \ldots\left(\alpha_{1}\right)_{k}^{\text {right }},
$$

and $\gamma_{k}^{\text {left }}$ is defined in a similar way. The relations associated to the twisted quiver are then given by

$$
I^{\prime}(I)=I_{1}^{\prime} \cup I_{2}^{\prime},
$$

where

$$
\begin{aligned}
& I_{1}^{\prime}=\left\{\alpha_{l}^{\text {right }} \varphi_{i k l}-\varphi_{j k l} \alpha_{k}^{\text {left }} \mid(\alpha: i \rightarrow j) \in Q_{1} ; k, l=1, \ldots, N\right\}, \\
& I_{2}^{\prime}=\left\{\sum_{r=1}^{l} \lambda_{r}\left(\gamma_{r}\right)_{k}^{z} \mid\left(\sum_{r=1}^{l} \lambda_{r} \gamma_{r}\right) \in I ; 1 \leq k \leq N ; z \in\{\text { left, right }\}\right\} .
\end{aligned}
$$

We think of the twisted quiver as a copy of the auxiliary quiver $Q^{\prime}$ at each vertex of $Q$, where the arrows in $Q$ are copied for each vertex in $Q^{\prime}$. The relations $I_{1}^{\prime}$ then tell us that in following an arrow in $Q$ and an arrow in $Q^{\prime}$ the order does not matter (up to changing the copy of the arrow). The relations $I_{2}^{\prime}$ tell us that for each vertex in $Q^{\prime}$ the corresponding copies of paths in $Q$ still satisfy the relations $I$. 
For instance, let

$$
Q=\bullet \stackrel{a}{\rightarrow} \bullet
$$

denote the $a$-morphism quiver, and let $Q^{\prime}$ denote the auxiliary quiver for $N=2$. Then $Q\left(Q^{\prime}\right)$, which might as well be interpreted as the quiver $Q^{\prime}$ doubled, looks as follows.

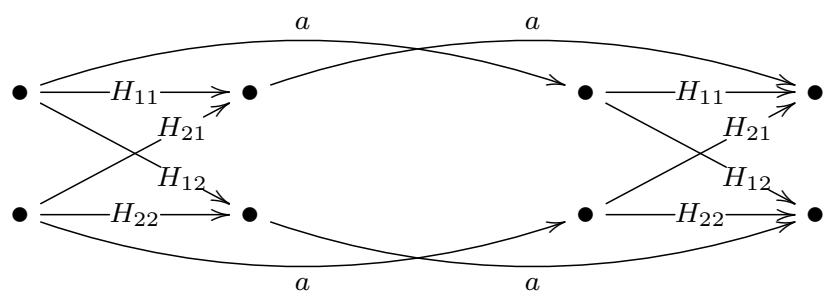

LEMMA 3.2.3. If the quiver $Q$ has no oriented cycles, the same holds for the twisted quiver $Q\left(Q^{\prime}\right)$.

Proof. The set of vertices of the quiver $Q\left(Q^{\prime}\right)$ is given as a copy, $Q^{\prime}(i)$, of $Q^{\prime}$ for each vertex $i \in Q_{0}$ of $Q$, and an arrow $\alpha: i \rightarrow j$ of $Q$ yields a set of arrows from the $i$ th copy $Q^{\prime}(i)$ to the $j$ th copy $Q^{\prime}(i)$.

Assume now that $Q\left(Q^{\prime}\right)$ has an oriented cycle $\gamma$. Then, for some $i_{0} \in Q_{0}$, the cycle $\gamma$ begins and ends in the $i_{0}$ th copy $Q^{\prime}\left(i_{0}\right)$ of $Q^{\prime}$. Now, $\gamma$ either stays entirely in $Q^{\prime}\left(i_{0}\right)$, or it passes through some $Q^{\prime}(i)$, with $i \neq i_{0}$, and then returns to $Q^{\prime}\left(i_{0}\right)$. In the first case, $Q^{\prime}$ would have to contain an oriented cycle, which is not the case. Hence, the second case holds. Then, however, $\gamma$ describes a sequence $Q^{\prime}\left(i_{0}\right), Q^{\prime}\left(i_{1}\right), \ldots, Q^{\prime}\left(i_{r}\right)=Q^{\prime}\left(i_{0}\right)$ of copies of $Q^{\prime}$ that it passes through, and this sequence is in fact defined by an oriented cycle in $Q$, a contradiction. Hence, $Q\left(Q^{\prime}\right)$ has no oriented cycles.

The natural target category of the extension of the embedding functor, which we construct in the next Section 3.2.2, can be identified with the category of representations of the twisted quiver.

LEMma 3.2.4. There is a canonical identification of categories

$$
(Q, I)-\operatorname{rep}_{A^{\prime}-\bmod } \simeq\left(Q\left(Q^{\prime}\right), H, I^{\prime}(I)\right)-\text { rep. }
$$

Proof. A representation of $\left(Q\left(Q^{\prime}\right), H\right)$ in particular consists of a representation of the auxiliary quiver for each vertex in $Q$. Also, for some fixed arrow $\alpha: i \rightarrow j$ of $Q$ the arrows $\alpha_{k}^{\text {left }}$ and $\alpha_{k}^{\text {right }}$ together with the relations $I_{1}^{\prime}$ comprise the data of a morphism of representations of $\left(Q^{\prime}, H\right)$. Moreover, the relations $I_{2}^{\prime}$ imply that these morphisms satisfy the relations $I$. The identification of $\left(Q^{\prime}, H\right)$ - rep with $A^{\prime}-\bmod$ thus induces the required equivalence. 


\subsubsection{The functor.}

The functor $\operatorname{Hom}(T, *)$ of [GRT16] extends to map quiver sheaves to representations of $Q$ in the category of representations of $\left(Q^{\prime}, H\right)$, which we identify with representations of $\left(Q\left(Q^{\prime}\right), H, I^{\prime}(I)\right)$.

Fix a topological type $\tau$ for a quiver sheaf. First, we recall Theorem 5.7 of [GRT16].

THEOREM 3.2.5. For $m \gg n \gg 0$, the functor

$$
\operatorname{Hom}(T, *): \operatorname{Coh}(X) \rightarrow A^{\prime}-\bmod
$$

is fully faithful on the full subcategory of $(n, \underline{L})$-regular sheaves of topological type $\tau_{i}$ for some $i \in Q_{0}$. More precisely, for $E$ in this subcategory the evaluation

$$
\eta_{E}: \operatorname{Hom}(T, E) \otimes_{A^{\prime}} T \rightarrow E
$$

is a natural isomorphism, and thus $\operatorname{Hom}(T, *)$ is left adjoint to the functor $* \otimes_{A^{\prime}} T$.

REMARK 3.2.6. Theorem 5.7 of [GRT16] is stated for one fixed topological type $\tau$. The main reason for this is to ensure the boundedness of the family of sheaves involved. The condition $m \gg n \gg 0$ can be satisfied for all of the finitely many $\tau_{i}$ simultaneously, providing us with an adjoint pair in this slightly more general case.

A module in the image of this functor can be written as

$$
\operatorname{Hom}(T, E)=\bigoplus_{j=1}^{N} H^{0}\left(E \otimes L_{j}^{n}\right) \oplus H^{0}\left(E \otimes L_{j}^{m}\right),
$$

where the arrow $\varphi_{i j}$ acts via the canonical map

$$
H^{0}\left(E \otimes L_{i}^{n}\right) \otimes_{k} H^{0}\left(L_{i}^{-n} \otimes L_{j}^{m}\right) \rightarrow H^{0}\left(E \otimes L_{j}^{m}\right) .
$$

Hence, the image of the functor (for a single $\tau$ ) lies in the full subcategory of representations of dimension vector $d^{\prime}=d^{\prime}(\tau, m, n)$ which reads as

$$
d_{v_{k}}^{\prime}=h^{0}\left(E \otimes L_{k}^{n}\right)=P_{E}^{L_{k}}(n), d_{w_{k}}^{\prime}=h^{0}\left(E \otimes L_{k}^{m}\right)=P_{E}^{L_{k}}(m) .
$$

Note that the Hilbert polynomials $P_{E}^{L_{k}}$, and hence the dimension vector, are fixed by the topological type $\tau$.

By abstract nonsense, using the naturalness of the evaluation, we obtain an extension of Theorem 3.2.5 to quiver sheaves. The target category of the induced embedding functor can be identified with the category of representations of the twisted quiver by Lemma 3.2.4.

TheOREM 3.2.7. For $m \gg n \gg 0$ the induced functor

$$
\operatorname{Hom}(T, *):(Q, I)-\operatorname{Coh}(X) \rightarrow\left(Q\left(Q^{\prime}\right), H, I^{\prime}(I)\right)-\text { rep }
$$

is fully faithful and exact on the full subcategory of $(n, \underline{L})$-regular quiver sheaves of topological type $\tau$. More precisely, for a quiver sheaf $\mathcal{E}$ in this subcategory the evaluation

$$
\eta_{\mathcal{E}}: \operatorname{Hom}(T, \mathcal{E}) \otimes_{A^{\prime}} T \rightarrow \mathcal{E}
$$


is a natural isomorphism, and thus $\operatorname{Hom}(T, *)$ is left adjoint to the functor $* \otimes_{A^{\prime}} T$. The image of the embedding lies in the full subcategory of representations of dimension vector $d(\tau, m, n)$.

Proof. By general nonsense, the functor of Theorem 3.2.5 extends to a functor

$$
\operatorname{Hom}(T, *):(Q, I)-\operatorname{Coh}(X) \rightarrow(Q, I)-\operatorname{rep}_{A^{\prime}-\bmod },
$$

and the target category is identified via Lemma 3.2.4. By definition, the functor is left-exact on $Q-\operatorname{Coh}(X)$, and on the subcategory it additionally is right-exact as it is a left-adjoint.

For a quiver sheaf $\mathcal{E}$ the decomposition reads as

$$
\operatorname{Hom}(T, \mathcal{E})=\bigoplus_{i \in Q_{0}} \bigoplus_{j=1}^{N} H^{0}\left(\mathcal{E}_{i} \otimes L_{j}^{n}\right) \oplus H^{0}\left(\mathcal{E}_{i} \otimes L_{j}^{m}\right),
$$

where the arrow $\varphi_{i k l}$ acts via the canonical map

$$
H^{0}\left(\mathcal{E}_{i} \otimes L_{k}^{n}\right) \otimes_{k} H^{0}\left(L_{k}^{-n} \otimes L_{l}^{m}\right) \rightarrow H^{0}\left(\mathcal{E}_{i} \otimes L_{l}^{m}\right),
$$

and the arrows $\alpha_{k}^{\text {left }}$ and $\alpha_{k}^{\text {right }}$ for some arrow $(\alpha: i \rightarrow j) \in Q_{1}$ act via

$$
H^{0}\left(\mathcal{E}_{i} \otimes L_{k}^{p}\right) \rightarrow H^{0}\left(\mathcal{E}_{j} \otimes L_{k}^{p}\right),
$$

where $p=n$ or $p=m$ respectively. Hence, $\operatorname{Hom}(T, \mathcal{E})$ has dimension vector $d=d(\tau, m, n)$ given as

$$
d_{v_{i k}}=h^{0}\left(\mathcal{E}_{i} \otimes L_{k}^{n}\right)=P_{\mathcal{E}_{i}}^{L_{k}}(n), d_{w_{i k}}=h^{0}\left(\mathcal{E}_{i} \otimes L_{k}^{m}\right)=P_{\mathcal{E}_{i}}^{L_{k}}(m) .
$$

Investigating the moduli functor requires a version of $\operatorname{Hom}(T, *)$ globalized to a functor between categories of flat families. First, recall the globalized functors in the setting of $A^{\prime}$-modules and ordinary sheaves as given in [GRT16].

Definition 3.2.8. Let $S$ denote any scheme.

Denote by flat $A^{\prime}(S)$ the category of $\mathcal{O}_{S} \otimes A^{\prime}$-modules on $S$ which are locally free as $\mathcal{O}_{S}$-modules. By flat ${ }_{S}(X \times S)$ we denote the category of $\mathcal{O}_{X \times S^{-}}$ modules which are flat over $S$.

The globalized functors

$$
\begin{array}{r}
\operatorname{Hom}^{\prime}(T, *): \operatorname{flat}_{S}(X \times S) \rightarrow \operatorname{flat}_{A^{\prime}}(S) \\
* \otimes_{A^{\prime}}^{\prime} T: \operatorname{flat}_{A^{\prime}}(S) \rightarrow \operatorname{flat}_{S}(X \times S)
\end{array}
$$

are defined as

$$
\operatorname{Hom}^{\prime}(T, \mathbb{E})=\left(p_{S}\right)_{*} \operatorname{Hom}\left(p_{X}^{*} T, \mathbb{E}\right), \mathbb{M} \otimes_{A^{\prime}}^{\prime} T=p_{S}^{*} \mathbb{M} \otimes_{\mathcal{O}_{X \times S} \otimes A^{\prime}} p_{X}^{*} T,
$$

where $p_{S}: X \times S \rightarrow S$ and $p_{X}: X \times S \rightarrow X$ are the canonical projections.

The globalized functor $\operatorname{Hom}^{\prime}(T, *)$ is also fully faithful, as shown in [GRT16] Proposition 5.8 by reducing to the fibers.

Proposition 3.2.9. For $m \gg n \gg 0$ the functor $\operatorname{Hom}^{\prime}(T, *)$ is fully faithful on the full subcategory of flat $_{S}(X \times S)$ consisting of flat families of $(n, \underline{L})$ regular sheaves of topological type $\tau$. 
Next, we want to extend these globalized functors to the case of a quiver $Q$ with relations $I$. Let $A$ denote the path algebra of the twisted quiver $\left(Q\left(Q^{\prime}\right), H, I^{\prime}(I)\right)$

Definition 3.2.10. Denote the category of representations of $Q$ in flat families as

$$
\operatorname{flat}_{S}(X \times S, Q, I)=(Q, I)-\operatorname{rep}_{f_{\text {flat }}(X \times S)} .
$$

By $\operatorname{flat}_{A}(S)$ we denote the category of $\mathcal{O}_{S} \otimes A$-modules on $S$ which are locally free as $\mathcal{O}_{S}$-modules.

Lemma 3.2.11. There is an equivalence of categories

$$
(Q, I)-\operatorname{rep}_{\text {flat }_{A^{\prime}}(S)} \simeq \operatorname{flat}_{A}(S),
$$

i.e. the category of representations of $(Q, I)$ in the category $\operatorname{flat}_{A^{\prime}}(S)$ is equivalent to the category $\operatorname{flat}_{A}(S)$.

Proof. This is the same identification as in Lemma 3.2.4, but twisted by $\mathcal{O}_{S}$.

Proposition 3.2.12. For $m \gg n \gg 0$ the functor $\operatorname{Hom}^{\prime}(T, *)$ induces a fully faithful functor

$$
\operatorname{Hom}^{\prime}(T, *): \operatorname{flat}_{S}(X \times S, Q, I)_{\tau} \rightarrow \operatorname{flat}_{A}(S)
$$

from the full subcategory of $(n, \underline{L})$-regular representations of topological type $\tau$ in flat $_{S}(X \times S)$ to flat $_{A}(S)$, which we will also denote by $\operatorname{Hom}^{\prime}(T, *)$. The corresponding functor induced by $* \otimes_{A^{\prime}}^{\prime} T$ will be denoted by $* \otimes_{A}^{\prime} T$.

Proof. The functorial nature of $\operatorname{Hom}^{\prime}(T, *)$ allows an extension to the category of representations of $Q$. The target of this extension can be identified with $\operatorname{flat}_{A}(S)$ by Lemma 3.2.11, and it is clear by abstract nonsense that the functor remains fully faithful.

We can give a description of the image of this functor.

Proposition 3.2.13. For $m \gg n \gg 0$ as in Proposition 3.2.12 and a flat family $\mathbb{M}$ of right-A-modules of dimension vector $d=d(\tau, m, n)$ over some scheme $B$ there exists a unique locally closed subscheme $i: B_{\tau}^{[\mathrm{reg}]} \subset B$ such that the following conditions hold.

(1) $i^{*} \mathbb{M} \otimes_{A^{\prime}}^{\prime} T$ is a family of $(n, \underline{L})$-regular quiver sheaves of topological type $\tau$ and satisfying the relations $I$ on $X$ which is flat over $B_{\tau}^{[\mathrm{reg}]}$, and the unit map

$$
\eta_{i^{*} \mathbb{M}}: i^{*} \mathbb{M} \rightarrow \operatorname{Hom}^{\prime}\left(T, i^{*} \mathbb{M} \otimes_{A^{\prime}}^{\prime} T\right)
$$

is an isomorphism.

(2) If $\sigma: S \rightarrow B$ is a subscheme such that $\sigma^{*} \mathbb{M} \simeq \operatorname{Hom}^{\prime}(T, \mathbb{E})$ for some family $\mathbb{E}$ of $(n, \underline{L})$-regular quiver sheaves of topological type $\tau$ which satisfy the relations $I$ on $X$ which is flat over $S$, then $\sigma$ factors through the embedding morphism $i$ and

$$
\mathbb{E} \simeq \sigma^{*} \mathbb{M} \otimes_{A^{\prime}}^{\prime} T .
$$


Proof. The identification of Lemma 3.2.11 allows us to consider $\mathbb{M}$ as a representation of $Q$ in the category of flat families of $A^{\prime}$-modules satisfying the relations $I$. Consider Proposition 5.9 in [GRT16], which is analogous to the present Proposition, and take $B_{\tau}^{[\mathrm{reg}]}$ to be the intersection of the corresponding closed subschemes provided for these families of $A^{\prime}$-modules. To see that this subscheme satisfies the intended assertions we can again trace back the identification of Lemma 3.2.11 and observe that all assertions may be checked at each vertex of $Q$, since the preservation of the relations is built into the functors. For the second assertion we also note that a morphism factors through a set of locally closed subschemes if and only if it factors through the intersection.

It seems natural to think of the relations as defining a closed subscheme. This is made precise in the following result. Just for the purpose of formulating this corollary, we let $A_{0}$ denote the path algebra for the case $I=\emptyset$, i.e. the path algebra of the quiver $\left(Q\left(Q^{\prime}\right), H, I_{1}^{\prime}\right)$.

Corollary 3.2.14. Suppose that we are given a topological type $\tau$, a set of relations $I$, and integers $m \gg n \gg 0$ such that Proposition 3.2.12 holds for $I=\emptyset$. For a flat family $\mathbb{M}$ of right- $A_{0}$-modules of dimension vector $d=d(\tau, m, n)$ over some scheme $B$ which restricts to a family $\mathbb{M}^{\prime}$ of modules which satisfy the relations $I_{2}^{\prime}=I_{2}^{\prime}(I)$ over some closed subscheme $B^{\prime} \subset B$ we have

$$
\left(B^{\prime}\right)_{\tau}^{[\mathrm{reg}], I}=B_{\tau}^{[\mathrm{reg}]} \cap B^{\prime} .
$$

Here, $B_{\tau}^{[\mathrm{reg}]} \subset B$ is the locally closed subscheme as in Proposition 3.2.12 in a version without relations, and $\left(B^{\prime}\right)_{\tau}^{[\mathrm{reg}], I}$ is the locally closed subscheme as in Proposition 3.2.12 in a version incorporating the relations $I$.

Proof. By construction, the restriction of the family $\mathbb{M}$ allows a description

$$
\left.\mathbb{M}\right|_{B[\mathrm{reg}]} \simeq \operatorname{Hom}^{\prime}(T, \mathbb{E}),
$$

where $\mathbb{E}$ is a family of quiver sheaves over $B^{[\mathrm{reg}]}=B_{\tau}^{[\mathrm{reg}]}$. Further restricted to $B_{\tau}^{[\mathrm{reg}]} \cap B^{\prime}$, the modules satisfy the relations $I_{2}^{\prime}$, so that, due to the construction of the functor, the quiver sheaves satisfy the relations $I$. This shows that

$$
B_{\tau}^{[\mathrm{reg}]} \cap B^{\prime} \subset\left(B^{\prime}\right)_{\tau}^{[\mathrm{reg}], I} .
$$

Conversely, $\mathbb{M}^{\prime}$ restricted to $\left(B^{\prime}\right)^{[\mathrm{reg}]}=\left(B^{\prime}\right)_{\tau}^{[\mathrm{reg}], I}$ allows a description

$$
\left.\mathbb{M}^{\prime}\right|_{\left(B^{\prime}\right)}{ }^{[\mathrm{reg}]}=\operatorname{Hom}^{\prime}\left(T, \mathbb{E}^{\prime}\right)
$$

for a family of quiver sheaves $\mathbb{E}^{\prime}$ which satisfy the relations $I$. Over the intersection $\left(B^{\prime}\right)^{[\mathrm{reg}]} \cap B^{[\mathrm{reg}]}$, the families $\mathbb{M}$ and $\mathbb{M}^{\prime}$ coincide, so that, by faithfulness of the functor $\operatorname{Hom}^{\prime}(T, *)$, the families $\mathbb{E}$ and $\mathbb{E}^{\prime}$ coincide as well. Thus, $\mathbb{E}$ and $\mathbb{E}^{\prime}$ glue to a flat family of right- $A$-modules over the subscheme

$$
\left(B^{\prime}\right)^{[\mathrm{reg}]} \cup B^{[\mathrm{reg}]} .
$$

But by maximality, this subscheme must equal $B^{[\mathrm{reg}]}$. 
Consider the functor

$$
\mathcal{M}^{\mathrm{reg}}(X, Q, I):(S c h / k)^{\mathrm{op}} \rightarrow \text { Sets },
$$

which assigns to a scheme $S$ the set of isomorphism classes of families of $(n, \underline{L})$-regular quiver sheaves on $X$ satisfying the relations $I$ of topological type $\tau$ which are flat over $S$.

For brevity denote $B=R_{d}\left(Q\left(Q^{\prime}\right), H, I^{\prime}(I)\right)_{\tau}^{[\text {reg }]}$, where we use the tautological family of modules $\mathbb{M}$ as explained in Appendix 1.2.3. Sending a morphism $f: S \rightarrow B$ to the family $f^{*}\left(\left.\mathbb{M}\right|_{B} \otimes_{A}^{\prime} T\right)$ defines a natural transformation

$$
g: \underline{B} \rightarrow \mathcal{M}^{\mathrm{reg}}(X, Q, I) .
$$

As in [ACK07] Theorem 4.5, the existence of the locally closed subscheme parameterizing the image of the embedding functor implies that this moduli functor is locally a quotient functor.

Proposition 3.2.15. There is a local isomorphism of functors

$$
\mathcal{M}^{\mathrm{reg}}(X, Q, I) \simeq \underline{B} / \underline{G},
$$

where $G=G_{d}$. This functor is induced by $g$.

Proof. The proof of [ACK07] Theorem 4.5 holds verbatim, where we use our version of the local isomorphism

$$
h^{\prime}: \underline{R} / \underline{G} \rightarrow \mathcal{M}_{A}
$$

as in Proposition 1.2.23 and our Proposition 3.2.13.

\subsection{Stability under embedding}

The next step in our program is to make sure that the embedding functor $\operatorname{Hom}(T, *)$ we constructed preserves stability. The technical cornerstone of this is a variant of the Le Potier-Simpson theorem for quiver sheaves and multi-Gieseker stability. We basically follow the reasoning of [GRT16], Sections 7 and 8 .

\subsubsection{The Le Potier-Simpson theorem.}

Suppose we are given a quiver $Q$ together with a (possibly empty) set of relations $I$, a stability condition $(\underline{L}, \sigma)$ and a topological type $\tau=\left(\tau_{i}\right)_{i \in Q_{0}}$.

We assume that $\sigma$ is a bounded stability condition, i.e. that the family of semistable quiver sheaves with respect to $\sigma$ which satisfy the relations is bounded. Using this we choose $p \gg 0$ such that all such quiver sheaves are $(p, \underline{L})$-regular. Furthermore, we assume that $\operatorname{dim}(E)=d$ for all sheaves of type $\tau_{i}$ for some $i \in Q_{0}$.

REMARK 3.3.1. Basically, the relations $I$ and $I_{2}^{\prime}$ are not of great importance in this section. This is due to the fact that the property of satisfying some given relation is inherited by quiver subsheaves, so that the notion of semistability is insensitive to relations. The only relevance these relations 
have is via the question of boundedness, which might only hold on the subfamily of semistable quiver sheaves which satisfy some relations.

On the other hand, the relations $I_{1}^{\prime}$ are essential for the technical step of Lemma 3.3.14.

Let us recall the Le Potier-Simpson estimate for the dimension of the space of global sections of a sheaf twisted by an ample line bundle. Because we use similar notation, we refer to the formulation in [GRT16] Theorem 7.1. Here, for a real number $x$ we use the notation $[x]_{+}=\max (x, 0)$.

TheOREM 3.3.2. Let $X$ denote a projective scheme, and $L$ a very ample line bundle on $X$. Let $E$ denote a purely d-dimensional sheaf on $X$ and define the number

$$
C_{E}^{L}=\left(r^{L}\right)^{2}+\frac{1}{2}\left(r^{L}+d\right)-1,
$$

where $r^{L}$ is the rank of $E$ with respect to $L$. Then, for any $n>0$ we have

$$
h^{0}\left(E \otimes L^{n}\right) \leq \frac{r^{L}-1}{d !}\left[\widehat{\mu}_{\max }^{L}(E)+C_{E}^{L}+n\right]_{+}^{d}+\frac{1}{d !}\left[\widehat{\mu}^{L}(E)+C_{E}^{L}+n\right]_{+}^{d} .
$$

To distinguish between strictly semistable and stable quiver sheaves we need more control over the destabilizing quiver subsheaves (compare with Lemma 2.13 in [GRT16]). Note that boundedness of $\sigma$ is not needed for the following proof.

Lemma 3.3.3. Suppose that $\mathcal{F} \subset \mathcal{E}$ is a destabilizing quiver subsheaf of a semistable quiver sheaf $\mathcal{E}$ of topological type $\tau$. Then $\mathcal{F} \subset \mathcal{E}$ is saturated and

$$
\mathcal{F} \oplus \mathcal{E} / \mathcal{F}
$$

is semistable with Hilbert polynomial $p_{\mathcal{E}}^{\sigma}$ and of topological type $\tau$.

Proof. $\mathcal{F}$ has the same Hilbert polynomial as $\mathcal{E}$ and its quiver subsheaves are quiver subsheaves of $\mathcal{E}$. It is thus again semistable. A similar reasoning using quotients shows that $\mathcal{E} / \mathcal{F}$ is semistable with Hilbert polynomial $p_{\mathcal{E}}^{\sigma}$ as well, which is a property inherited by the direct sum. The assertion about the topological type is implied by its additivity.

To see that $\mathcal{F}$ is saturated consider the inequalities

$$
p_{\mathcal{F}}^{\sigma}(n)=\frac{\sum_{i, j} \sigma_{i, j} h^{0}\left(\mathcal{F}_{i} \otimes L_{j}^{n}\right)}{r^{\sigma}(\mathcal{F})} \leq \frac{\sum_{i, j} \sigma_{i, j} h^{0}\left(\mathcal{G}_{i} \otimes L_{j}^{n}\right)}{r^{\sigma}(F)}=p_{\mathcal{G}}^{\sigma}(n) \leq p_{\mathcal{E}}^{\sigma}(n)
$$

for $n \gg 0$, where $\mathcal{G} \subset \mathcal{E}$ is the saturation of $\mathcal{F}$, and the last inequality holds by semistability. The inequalities are thus actually equalities, and in particular for each $\mathrm{i} \in Q_{0}$ we have

$$
H^{0}\left(\mathcal{F}_{i} \otimes L_{j}^{n}\right)=H^{0}\left(\mathcal{G}_{i} \otimes L_{j}^{n}\right)
$$

for at least one index $j=j(i)$ such that $\sigma_{i j}$ does not vanish. For $n \gg 0$ the sheaves $\mathcal{G}_{i} \otimes L_{j}^{n}$ are globally generated, so $\mathcal{G}_{i} \subset \mathcal{F}_{i}$ for all $i \in Q_{0}$, which shows $\mathcal{F}=\mathcal{G}$.

We are ready to proof the Le Potier-Simpson theorem for quiver sheaves (compare with [GRT16], Theorem 7.2). Recall that we assume $\sigma$ to be bounded, and $\sigma$-semistable quiver sheaves to be $(p, \underline{L})$-regular. 
THEOREM 3.3.4. For an integer $n \gg p \gg 0$ the following assertions are equivalent for a purely $d$-dimensional quiver sheaf $\mathcal{E}$ of topological type $\tau$ which satisfies the relations $I$.

(1) $\mathcal{E}$ is semistable.

(2) $\mathcal{E}$ is $(p, \underline{L})$-regular and for all quiver subsheaves $\mathcal{F} \subset \mathcal{E}$ we have

$$
\frac{\sum_{i \in Q_{0}} \sum_{j=1}^{N} \sigma_{i j} h^{0}\left(\mathcal{F}_{i} \otimes L_{j}^{n}\right)}{r^{\sigma}(\mathcal{F})} \leq p_{\mathcal{E}}^{\sigma}(n) .
$$

(3) $\mathcal{E}$ is $(p, \underline{L})$-regular and the above inequality holds for all saturated quiver subsheaves $\mathcal{F} \subset \mathcal{E}$ such that

$$
\widehat{\mu}^{\sigma}(\mathcal{F}) \geq \widehat{\mu}^{\sigma}(\mathcal{E}) .
$$

The same statement holds for stable quiver sheaves and strict inequality. Moreover, equality holds for a quiver subsheaf $\mathcal{F} \subset \mathcal{E}$ if and only if $\mathcal{F}$ is destabilizing.

Proof. The set of $(p, \underline{L})$-regular sheaves of topological type $\tau_{i}$ for some $i \in Q_{0}$ is bounded. Hence we can bound

$$
\widehat{\mu}_{\max }^{L_{j}}(F) \leq C_{1}
$$

for all such sheaves $F$ by a constant $C_{1}>0$. Recall that

$$
C_{E}^{L}=\frac{1}{2} r^{L_{j}}(E)\left(r^{L_{j}}(E)+\operatorname{dim}(E)\right),
$$

the constant used in the Le Potier-Simpson estimate, is fixed by the topological type of the sheaf $E$. Hence we may set

$$
\bar{C}=\max \left(C_{\mathcal{E}_{i}}^{L_{j}}\right),
$$

where $\mathcal{E}$ is any quiver sheaf of topological type $\tau$.

We now claim that there exists a constant $C_{2}>0$ which satisfies the following assertions.

(1) For all $(p, \underline{L})$-regular quiver sheaves $\mathcal{E}$ of topological type $\tau$ we have

$$
-\widehat{\mu}^{\sigma}(\mathcal{E})+1 \leq C_{2} .
$$

(2) For any $(p, \underline{L})$-regular quiver sheaf $\mathcal{E}$ of topological type $\tau$, all nonempty subsets $I \subset Q_{0}$ and all integers $0 \leq e_{i, j} \leq \mathrm{rk}^{L_{j}}\left(\mathcal{E}_{i}\right)$ the inequality

$$
\begin{aligned}
& \sum_{i \in I^{c}, j}\left(\sigma_{i j} e_{i j} C_{1}\right)+\sum_{i \in I, j}\left(\frac{\sigma_{i j}}{d !}\left(e_{i j}-1\right)\left(C_{1}+\bar{C}\right)+\left(-C_{2}+\bar{C}\right)\right) \\
& \leq\left(\widehat{\mu}^{\sigma}(\mathcal{E})-1\right) \sum_{i \in Q_{0}, j} \sigma_{i j} e_{i j}
\end{aligned}
$$

holds, where $I^{c}=Q_{0} \backslash I$ denotes the complement, and the sums are simultaneously taken over $j=1, \ldots, N$.

This is possible because of the boundedness of the family of involved quiver sheaves, and because the parameters occurring in the second inequality, besides $C_{2}$, are either fixed beforehand or only vary within a finite set.

Consider the set $S$ of sheaves $F$ which are saturated subsheaves $F \subset E$ of a $(p, \underline{L})$-regular sheaf $E$ of topological type $\tau$ and such that $\widehat{\mu}^{L_{j}}(F) \geq-C_{2}$ 
for some $j$. This set is bounded by the Grothendieck Lemma for sheaves (because the subsheaves are saturated we can equivalently consider the quotients and apply [HL10] Lemma 1.7.9).

Furthermore, consider the set $S^{\prime}$ of quiver sheaves $\mathcal{F}$ which are saturated quiver subsheaves $\mathcal{F} \subset \mathcal{E}$ of some $(p, \underline{L})$-regular quiver sheaf $\mathcal{E}$ of topological type $\tau$ and such that $\widehat{\mu}^{\sigma}(F) \geq-C_{2}$. This set is bounded as well according to the Grothendieck Lemma 3.1.21 for quiver sheaves.

To shorten notation we introduce the numbers

$$
D_{1}=C_{1}+\bar{C}, D_{2}=-C_{2}+\bar{C} .
$$

Now we further claim that for $n \gg p$ the following assertions hold.

(1) Given any quiver sheaf $\mathcal{F}$ which consists of sheaves in $S$ or is in $S^{\prime}$ and any quiver sheaf $\mathcal{E}$ which is $(p, \underline{L})$-regular and of topological type $\tau$ we have that

$$
p_{\mathcal{F}}^{\sigma}(n) \sim p_{\mathcal{E}}^{\sigma}(n) \Leftrightarrow p_{\mathcal{F}}^{\sigma} \sim p_{\mathcal{E}}^{\sigma},
$$

where $\sim$ is one of the relations $=, \leq$ or $<$.

(2) All $F \in S$ are $(n, \underline{L})$-regular.

(3) The quiver sheaves $\mathcal{F} \in S^{\prime}$ are all $(n, \underline{L})$-regular.

(4) $n>C_{2}-\bar{C}$

(5) For all quiver subsheaves $\mathcal{F} \subset \mathcal{E}$ of some $(p, \underline{L})$-regular quiver sheaf $\mathcal{E}$ of topological type $\tau$ and all non-empty subsets $I \subset Q_{0}$ we have

$$
\begin{aligned}
& \sum_{i \in I^{c}, j} \sigma_{i j} P_{\mathcal{F}_{i}}^{L_{j}}(n)+\sum_{i \in I, j} \frac{\sigma_{i j}}{d !}\left(\left(r^{L_{j}}\left(\mathcal{F}_{i}\right)-1\right)\left(D_{1}+n\right)^{d}+\left(D_{2}+n\right)^{d}\right) \\
& \leq r^{\sigma}(\mathcal{F})\left(p_{\mathcal{E}}^{\sigma}(n)-1\right) .
\end{aligned}
$$

The first three assertions are true because the involved families of quiver sheaves are bounded, and the fourth assertion holds because the right hand side is just a constant. To see that the last assertion holds note that, after bringing $r^{\sigma}(\mathcal{F})$ to the other side, the left hand side is a polynomial in $n$ with leading coefficient $\frac{1}{d !}$ and second coefficient

$$
\frac{\sum_{i \in I^{c}, j}\left(\sigma_{i j} \alpha_{d-1}^{L_{j}}\left(\mathcal{F}_{i}\right)\right)+\sum_{i \in I, j}\left(\frac{\sigma_{i j}}{d !}\left(\mathrm{rk}^{L_{j}}\left(\mathcal{F}_{i}\right)-1\right) D_{1}+D_{2}\right)}{r^{\sigma}(\mathcal{F})} .
$$

Then we may estimate

$$
\alpha_{d-1}^{L_{j}}\left(\mathcal{F}_{i}\right)=\alpha_{d}^{L_{j}}\left(\mathcal{F}_{i}\right) \widehat{\mu}^{L_{j}}\left(\mathcal{F}_{i}\right) \leq \operatorname{rk}^{L_{j}}\left(\mathcal{F}_{i}\right) \widehat{\mu}_{\max }^{L_{j}}\left(\mathcal{E}_{i}\right) \leq \operatorname{rk}^{L_{j}}\left(\mathcal{F}_{i}\right) C_{1}
$$

to arrive at an expression as in the second condition for $C_{2}$. Thus for large $n$ the inequality of polynomials holds as claimed, and there are only finitely many such conditions.

We now prove 1.) $\Rightarrow 2$.). Let $\mathcal{E}$ denote some semistable quiver sheaf satisfying the relations, which is hence $(p, \underline{L})$-regular by assumption. Consider a quiver subsheaf $\mathcal{F} \subset \mathcal{E}$. Note that if $\mathcal{G}$ denotes the saturation of $\mathcal{F}$ in $\mathcal{E}$ we have

$$
\frac{\sum_{i \in Q_{0}} \sum_{j=1}^{N} \sigma_{i j} h^{0}\left(\mathcal{F}_{i} \otimes L_{j}^{n}\right)}{r^{\sigma}(\mathcal{F})} \leq \frac{\sum_{i \in Q_{0}} \sum_{j=1}^{N} \sigma_{i j} h^{0}\left(\mathcal{G}_{i} \otimes L_{j}^{n}\right)}{r^{\sigma}(\mathcal{G})},
$$


so we may assume that $\mathcal{F}$ is saturated without loss of generality.

Let $I \subset Q_{0}$ denote the set of vertices such that

$$
\widehat{\mu}^{L_{j}}\left(\mathcal{F}_{i}\right)<-C_{2}
$$

for all $j=1, \ldots, N$. Again, let $I^{c}$ denote the complement of this set.

Note that for $i \in I^{c}$ we clearly have $\mathcal{F}_{i} \in S$, so $\mathcal{F}_{i}$ is $(n, \underline{L})$-regular by the choice of $n$. If $i \in I$ we know

$$
\widehat{\mu}_{\max }^{L_{j}}\left(\mathcal{F}_{i}\right) \leq \widehat{\mu}_{\max }^{L_{j}}\left(\mathcal{E}_{i}\right) \leq C_{1}
$$

and $C_{\mathcal{F}_{i}}^{L_{j}} \leq C_{\mathcal{E}_{i}}^{L_{j}} \leq \bar{C}$. Thus the Le Potier-Simpson estimate (Theorem 3.3.2) tells us

$$
\begin{aligned}
h^{0}\left(\mathcal{F}_{i} \otimes L_{j}^{n}\right) & \leq \frac{r^{L_{j}}\left(\mathcal{F}_{i}\right)-1}{d !}\left[\widehat{\mu}_{\max }^{L_{j}}\left(\mathcal{F}_{i}\right)+C_{\mathcal{F}_{i}}^{L_{j}}+n\right]_{+}^{d} \\
& +\frac{1}{d !}\left[\widehat{\mu}^{L_{j}}\left(\mathcal{F}_{i}\right)+C_{\mathcal{F}_{i}}^{L_{j}}+n\right]_{+}^{d} \\
& \leq \frac{r^{L_{j}}\left(\mathcal{F}_{i}\right)-1}{d !}\left(D_{1}+n\right)^{d}+\frac{1}{d !}\left(D_{2}+n\right)^{d}
\end{aligned}
$$

by our choice of constants and $n$.

Now we distinguish two cases.

If $I=\emptyset$, i.e. $I^{c}=Q_{0}$, we have that

$$
\frac{\sum_{i \in Q_{0}} \sum_{j=1}^{N} \sigma_{i j} h^{0}\left(\mathcal{F}_{i} \otimes L_{j}^{n}\right)}{r^{\sigma}(\mathcal{F})}=p_{\mathcal{F}}^{\sigma}(n) \leq p_{\mathcal{E}}^{\sigma}(n),
$$

where the first equality holds because all $\mathcal{F}_{i}$ are $(n, \underline{L})$-regular and the inequality is equivalent to $p_{\mathcal{F}}^{\sigma} \leq p_{\mathcal{E}}^{\sigma}$, which holds by semistability of $\mathcal{E}$.

If $\mathcal{E}$ is stable, the inequality is strict by the same argument.

On the other hand, if $I \neq \emptyset$ we may rewrite

$$
\begin{aligned}
& \sum_{i \in Q_{0}} \sum_{j=1}^{N} \sigma_{i j} h^{0}\left(\mathcal{F}_{i} \otimes L_{j}^{n}\right)=\sum_{i \in I^{c}, j} \sigma_{i j} h^{0}\left(\mathcal{F}_{i} \otimes L_{j}^{n}\right)+\sum_{i \in I, j} \sigma_{i j} h^{0}\left(\mathcal{F}_{i} \otimes L_{j}^{n}\right) \\
& \leq \sum_{i \in I^{c}, j} \sigma_{i j} P_{\mathcal{F}_{i}}^{L_{j}}(n)+\sum_{i \in I, j} \frac{\sigma_{i j}}{d !}\left(\left(r^{L_{j}}\left(\mathcal{F}_{i}\right)-1\right)\left(D_{1}+n\right)^{d}+\left(D_{2}+n\right)^{d}\right)
\end{aligned}
$$

and the right hand side is strictly smaller than $p_{\mathcal{E}}^{\sigma}(n) r^{\sigma}(\mathcal{F})$ by choice of $n$.

That 2.$) \Rightarrow 3$.) holds is very obvious. It thus remains to check the direction 3.) $\Rightarrow 1$.).

Let $\mathcal{E}$ denote any $(p, \underline{L})$-regular quiver sheaf of topological type $\tau$, and let $\mathcal{F} \subset \mathcal{E}$ denote a quiver subsheaf. Without loss of generality we assume that $\mathcal{F}$ is saturated.

If $\widehat{\mu}^{\sigma}(\mathcal{F})<\widehat{\mu}^{\sigma}(\mathcal{E})$ then clearly $\mathcal{F}$ does not destabilize. If on the other hand $\widehat{\mu}^{\sigma}(\mathcal{F}) \geq \widehat{\mu}^{\sigma}(\mathcal{E}) \geq-C_{2}$, where the latter inequality holds by choice of $C_{2}$, 
we know that $\mathcal{F}$ is contained in $S^{\prime}$ and is hence $(n, \underline{L})$-regular. Thus

$$
p_{\mathcal{F}}^{\sigma}(n)=\frac{\sum_{i \in Q_{0}} \sum_{j=1}^{N} \sigma_{i j} h^{0}\left(\mathcal{F}_{i} \otimes L_{j}^{n}\right)}{r^{\sigma}(\mathcal{F})} \leq p_{\mathcal{E}}^{\sigma}(n)
$$

for $n \gg 0$, which implies $p_{\mathcal{F}}^{\sigma} \leq p_{\mathcal{E}}^{\sigma}$.

If the inequality in 2.) is strict the above inequality is also strict, and thus $\mathcal{E}$ is stable.

It remains to show the addendum.

Consider some quiver subsheaf $\mathcal{F} \subset \mathcal{E}$ and its saturation $\mathcal{G} \subset \mathcal{E}$. Recall the argument of 1.) $\Rightarrow 2$.), and note that most of it does not actually make use of the assumption of 1.). Only the case $I=\emptyset$ is relevant though. Because if $I \neq \emptyset$ holds for $\mathcal{G}$, the inequality in 2.) is strict, so $\mathcal{G}$ and thus $\mathcal{F}$ can not be destabilizing.

If $\mathcal{F}$ is destabilizing it is saturated by Lemma 3.3.3, and hence consists of sheaves in the family $S$. This implies that the desired equality in 2.) is equivalent to the equality $p_{\mathcal{F}}^{\sigma}=p_{\mathcal{E}}^{\sigma}$. Conversely, if equality holds in 2.) we have that

$$
\begin{aligned}
p_{\mathcal{E}}^{\sigma}(n)=p_{\mathcal{F}}^{\sigma}(n) & =\frac{\sum_{i \in Q_{0}} \sum_{j=1}^{N} \sigma_{i j} h^{0}\left(\mathcal{F}_{i} \otimes L_{j}^{n}\right)}{r^{\sigma}(\mathcal{F})} \\
& \leq \frac{\sum_{i \in Q_{0}} \sum_{j=1}^{N} \sigma_{i j} h^{0}\left(\mathcal{G}_{i} \otimes L_{j}^{n}\right)}{r^{\sigma}(\mathcal{G})}=p_{\mathcal{E}}^{\sigma}(n) .
\end{aligned}
$$

Note that as $\mathcal{G}$ consists of sheaves in $S$ it in particular is $(n, \underline{L})$-regular, so that an argument as in the proof of Lemma 3.5.7 shows that $\mathcal{F}$ is saturated. Hence $\mathcal{F}=\mathcal{G}$ and equality in 2.) is equivalent to $p_{\mathcal{F}}^{\sigma}=p_{\mathcal{E}}^{\sigma}$.

Actually, a slight reformulation of the Theorem of Le Potier-Simpson is needed.

COROLlary 3.3.5. For $n \gg p \gg 0$, the following assertions are equivalent for any pure quiver sheaf $\mathcal{E}$ which is of topological type $\tau$ and which satisfies the relations $I$.

(1) $\mathcal{E}$ is semistable.

(2) $\mathcal{E}$ is $(p, \underline{L})$-regular and for all quiver subsheaves $\mathcal{F} \subset \mathcal{E}$ it holds

$$
\sum_{i, j} \sigma_{i j} h^{0}\left(\mathcal{F}_{i} \otimes L_{j}^{n}\right) P_{\mathcal{E}}^{\sigma} \leq P_{\mathcal{E}}^{\sigma}(n) P_{\mathcal{F}}^{\sigma}
$$

(3) $\mathcal{E}$ is $(p, \underline{L})$-regular and the above inequality holds for all saturated quiver subsheaves $\mathcal{F} \subset \mathcal{E}$ such that $\widehat{\mu}^{\sigma}(\mathcal{F}) \geq \widehat{\mu}^{\sigma}(\mathcal{E})$.

Moreover, for semistable $\mathcal{E}$ and destabilizing $\mathcal{F} \subset \mathcal{E}$, equality in 2) holds if and only if $\mathcal{F}$ is destabilizing.

Proof. As in the proof of [GRT16] Corollary 7.3, we may rewrite the inequality concerning the polynomials $P_{\mathcal{E}}^{\sigma}$ and $P_{\mathcal{F}}^{\sigma}$ as an inequality concerning the leading coefficients. The reformulated assertions are then equivalent by the Le Potier-Simpson Theorem 3.3.4. 


\subsubsection{Semistability under embedding.}

We now want to show that a quiver sheaf $\mathcal{E}$ is semistable if and only if its image under the embedding functor $\operatorname{Hom}(T, \mathcal{E})$ is semistable. Recall that we still assume $\sigma$ to be a bounded stability condition given the relations $I$. To that end, we start by fixing integers $m \gg n \gg p \gg 0$ that satisfy certain technical conditions. Later, we will show that the embedding functor defined by these integers has the desired property of preserving stability.

To formulate the technical conditions we first need some definitions.

Definition 3.3.6. Let $E$ denote any $(n, \underline{L})$-regular sheaf of topological type $\tau_{i}$ for some $i \in Q_{0}$. Consider the evaluation map

$$
\mathrm{ev}_{j}: H^{0}\left(E \otimes L_{j}^{n}\right) \otimes L_{j}^{-n} \rightarrow E \rightarrow 0 .
$$

For any subspace $V_{j}^{\prime} \subset H^{0}\left(E \otimes L_{j}^{n}\right)$ denote the image and kernel of the map induced by $\mathrm{ev}_{j}$ as

$$
0 \rightarrow F_{j}^{\prime} \rightarrow V_{j}^{\prime} \otimes L_{j}^{-n} \rightarrow E_{j}^{\prime} \rightarrow 0 .
$$

Furthermore, consider

$$
0 \rightarrow K \rightarrow \bigoplus_{j=1}^{N} V_{j}^{\prime} \otimes L_{j}^{-n} \rightarrow \sum_{j=1}^{N} E_{j}^{\prime} \rightarrow 0 .
$$

Denote by $S_{\mathrm{ev}}$ the family of sheaves $E_{j}^{\prime}, F_{j}^{\prime}, \sum_{j=1}^{N} E_{j}^{\prime}$ and $K$ that arise in such a way.

Definition 3.3.7. Let $S$ denote the family of quiver sheaves $\mathcal{F}$ such that $\mathcal{F} \subset \mathcal{E}$ is a saturated quiver subsheaf, where $\mathcal{E}$ is $(p, \underline{L})$-regular of topological type $\tau$, and such that $\widehat{\mu}^{\sigma}(\mathcal{F}) \geq \widehat{\mu}^{\sigma}(\mathcal{E})$.

We claim that there are integers $m \gg n \gg p \gg 0$ satisfying the following assertions, which we will keep fixed in the following arguments.

(1) All semistable quiver sheaves of topological type $\tau$ which satisfy the relations $I$ are $(p, \underline{L})$-regular.

(2) The Le Potier-Simpson corollary 3.3.5 holds.

(3) $L_{j}^{-n}$ is $(m, \underline{L})$-regular for all $j=1, \ldots, N$.

(4) All quiver sheaves in $S$ and all sheaves in $S_{\text {ev }}$ are $(m, \underline{L})$-regular.

(5) For all quiver sheaves $\mathcal{E}$ of topological type $\tau$, for all integers $c_{i j}$ between 0 and $P_{\mathcal{E}_{i}}^{L_{j}}(n)$ and for all quiver sheaves $\mathcal{F}$ which are in $S$ or consist of sheaves in $S_{\text {ev }}$ the relation of polynomials

$$
P_{\mathcal{E}}^{\sigma} \sum_{i \in Q_{0}} \sum_{j=1}^{N} \sigma_{i j} c_{i j} \sim P_{\mathcal{E}}^{\sigma}(m) P_{\mathcal{F}}^{\sigma}
$$

holds if and only if the relation

$$
P_{\mathcal{E}}^{\sigma}(m) \sum_{i \in Q_{0}} \sum_{j=1}^{N} \sigma_{i j} c_{i j} \sim P_{\mathcal{E}}^{\sigma}(m) P_{\mathcal{F}}^{\sigma}(m)
$$

holds, where $\sim \in\{=, \leq,<\}$.

(6) The functor $\operatorname{Hom}(T, *)$ is an embedding, i.e. Theorem 3.2.7 holds. 
The first assertion can be achieved because the family of semistable quiver sheaves is bounded by assumption. The second and the sixth assertions hold by the statements of the Corollary and the Theorem, and the third assertion is easily achieved because there are only finitely many $L_{j}$. To guarantee the fourth and fifth assertion note that $S$ is bounded by the Grothendieck Lemma 3.1.21, and $S_{\text {ev }}$ is also bounded (see [GRT16] Definition 8.3).

Recall that the embedding functor $\operatorname{Hom}(T, *)$ maps any $(n, \underline{L})$-regular quiver sheaf $\mathcal{E}$ to a representation of the twisted quiver $Q\left(Q^{\prime}\right)$ of dimension vector $d$, given by

$$
d_{i j 1}=h^{0}\left(\mathcal{E}_{i} \otimes L_{j}^{n}\right)=P_{\mathcal{E}_{i}}^{L_{j}}(n), d_{i j 2}=h^{0}\left(\mathcal{E}_{i} \otimes L_{j}^{m}\right)=P_{\mathcal{E}_{i}}^{L_{j}}(m) .
$$

For brevity, we let the indices $(i j 1)$ and $(i j 2)$ refer to the vertices $v_{i j}$ and $w_{i j}$ respectively. We also define a stability condition $\theta=\theta(\sigma, d)$ on $Q\left(Q^{\prime}\right)$ by

$$
\theta_{i j 1}=\frac{\sigma_{i j}}{\sum_{k \in Q_{0}} \sum_{l=1}^{N} \sigma_{k l} d_{k l 1}}, \theta_{i j 2}=\frac{-\sigma_{i j}}{\sum_{k \in Q_{0}} \sum_{l=1}^{N} \sigma_{k l} d_{k l 2}} .
$$

For a representation $M$ of the twisted quiver $Q\left(Q^{\prime}\right)$ we use the notation

$$
M=\bigoplus_{i \in Q_{0}} \bigoplus_{j=1}^{N} V_{i j} \oplus W_{i j},
$$

where $V_{i j}$ is the value of $M$ at the vertex $v_{i j}$ and $W_{i j}$ is the value of $M$ at the vertex $w_{i j}$. The maps associated to the arrows $\varphi_{i k l}$ are denoted as

$$
\phi_{i k l}: V_{i k} \otimes H_{k l} \rightarrow W_{i l},
$$

and the maps associated to the arrows $\alpha_{k}^{\text {left }}$ and $\alpha_{k}^{\text {right }}$ are denoted as $A_{k}$ and $B_{k}$ in a similar fashion. We will also use obvious variants of this notation, for example for some other representation $M^{\prime}$.

Using this notation we may rewrite

$$
\theta(M)=\sum_{i, j}\left(\theta_{i j 1} \operatorname{dim}\left(V_{i j}\right)+\theta_{i j 2} \operatorname{dim}\left(W_{i j}\right)\right) .
$$

Note that for a representation $M$ of dimension vector $d$ we have $\theta(M)=0$, so that $M$ is semistable if and only if $\theta(N) \leq 0$ holds for all subrepresentations $N \subset M$.

To relate stability of representations to stability of quiver sheaves a slightly different notion of the slope is useful.

Definition 3.3.8. Let $M=\oplus_{i, j} V_{i j} \oplus W_{i j}$ denote a representation of $Q\left(Q^{\prime}\right)$ such that $\sum_{i, j} \sigma_{i j} \operatorname{dim}\left(W_{i j}\right)$ or $\sum_{i, j} \sigma_{i j} \operatorname{dim}\left(V_{i j}\right)$ are non-zero. Then we define the (auxiliary) slope of $M$ as

$$
\mu^{\prime}(M)=\frac{\sum_{i, j} \sigma_{i j} \operatorname{dim}\left(V_{i j}\right)}{\sum_{i, j} \sigma_{i j} \operatorname{dim}\left(W_{i j}\right)} \in[0, \infty] .
$$


Lemma 3.3.9. Let $M$ denote a representation of $Q\left(Q^{\prime}\right)$ of dimension vector $d$, and let $M^{\prime}$ denote a representation such that $\sum_{i, j} \sigma_{i j} \operatorname{dim}\left(W_{i j}^{\prime}\right) \neq 0$.

Then $\theta\left(M^{\prime}\right) \leq 0$ if and only if $\mu^{\prime}\left(M^{\prime}\right) \leq \mu^{\prime}(M)$.

The same assertion holds if we replace $\leq$ by $<$.

Proof. This can be shown by an elementary argument as in the proof of [GRT16] Lemma 8.6.

There are subrepresentations, called non-degenerate, on which the auxiliary slope is well-defined. For representations in the image of the embedding functor, it suffices to check semistability on such subrepresentations. Degenerate subrepresentations always destabilize.

Definition 3.3.10. A representation $M$ of $Q\left(Q^{\prime}\right)$ is called degenerate if $V_{i j}=0$ for all $i \in Q_{0}$ and $j \in\{1, \ldots, N\}$ and $W_{i j}=0$ for all $i$ and $j$ such that $\sigma_{i j}=0$.

Lemma 3.3.11. Let $M=\operatorname{Hom}(T, \mathcal{E})$ denote the representation of $Q\left(Q^{\prime}\right)$ of dimension vector $d$ given by a $(n, \underline{L})$-regular quiver sheaf $\mathcal{E}$ of topological type $\tau$. Then the following holds.

(1) If a representation $M^{\prime}$ is non-degenerate we have

$$
\sum_{i, j} \sigma_{i j} \operatorname{dim}\left(W_{i j}^{\prime}\right) \neq 0
$$

and $\mu^{\prime}\left(M^{\prime}\right)$ is well-defined.

(2) $M$ is $\theta$-semistable if and only if $\mu^{\prime}\left(M^{\prime}\right) \leq \mu^{\prime}(M)$ for all non-degenerate $M^{\prime} \subset M$.

(3) Suppose that $M$ is $\theta$-semistable. Then $M^{\prime} \subset M$ is destabilizing if and only if $M^{\prime}$ is degenerate or $M$ is non-degenerate and $\mu^{\prime}(M)=\mu^{\prime}\left(M^{\prime}\right)$.

Proof. Write $M=\operatorname{Hom}(T, \mathcal{E})=\bigoplus_{i, j} H^{0}\left(\mathcal{E}_{i} \otimes L_{j}^{n}\right) \oplus H^{0}\left(\mathcal{E}_{i} \otimes L_{j}^{m}\right)$. Recall that in this representation the arrows $\varphi_{i k l}$ are equipped with maps

$$
\phi_{i k l}: H^{0}\left(\mathcal{E}_{i} \otimes L_{k}^{n}\right) \otimes H^{0}\left(L_{l}^{m} \otimes L_{k}^{-n}\right) \rightarrow H^{0}\left(\mathcal{E}_{i} \otimes L_{l}^{m}\right) .
$$

By our assumption on $m$ and $n$ the sheaf $L_{l}^{m} \otimes L_{k}^{-n}$ is globally generated, so as in the proof of [GRT16] Lemma 8.8, if $\phi_{i k l}(s \otimes h)=0$ for all $h$ then $s=0$. Thus the first assertion can be shown as in the case of sheaves.

The rest follows by Lemma 3.3.9 once we note that $\theta\left(M^{\prime}\right)=0$ if $M^{\prime}$ is degenerate. Compare with the proof of [GRT16] Lemma 8.8. 
We consider the notion of subordinate subrepresentations and tight representations as in [GRT16] Definition 8.10.

Definition 3.3.12. Let $M^{\prime}$ and $M^{\prime \prime}$ denote subrepresentations of some representation $M$ of $Q\left(Q^{\prime}\right)$.

(1) We say that $M^{\prime}$ is subordinate to $M^{\prime \prime}$ if

$$
V_{i j}^{\prime} \subset V_{i j}^{\prime \prime} \text { and } W_{i j}^{\prime \prime} \subset W_{i j}^{\prime}
$$

holds for all $i \in Q_{0}$ and all $j=1, \ldots, N$. We denote this by

$$
M^{\prime} \preceq M^{\prime \prime} .
$$

(2) A subrepresentation $M^{\prime}$ is called tight if whenever $M^{\prime} \preceq M^{\prime \prime}$ for another subrepresentation of $M$ we have

$$
V_{i j}^{\prime}=V_{i j}^{\prime \prime} \text { and } W_{i j}^{\prime}=W_{i j}^{\prime \prime}
$$

for all indices $i$ and $j$ such that $\sigma_{i j} \neq 0$.

LEMMA 3.3.13. Let $M^{\prime}$ and $M^{\prime \prime}$ denote subrepresentations of some representation $M$ such that $\mu^{\prime}\left(M^{\prime}\right)$ and $\mu^{\prime}\left(M^{\prime \prime}\right)$ are well-defined. If $M^{\prime} \preceq M^{\prime \prime}$ we have

$$
\mu^{\prime}\left(M^{\prime}\right) \leq \mu^{\prime}\left(M^{\prime \prime}\right)
$$

Moreover, if $M^{\prime}$ is tight equality holds.

Proof. This is elementary. Compare with [GRT16] Lemma 8.11.

Interestingly, the next Lemma needs the relations $I_{1}^{\prime}$ on the twisted quiver.

Lemma 3.3.14. Let $M$ satisfy the relations $I_{1}^{\prime}$, and consider any subrepresentation $M^{\prime} \subset M$. Then

$$
M^{\prime} \preceq M^{\prime \prime}
$$

for a tight subrepresentation $M^{\prime \prime} \subset M$.

Proof. Adapting the proof of [GRT16] Lemma 8.12 we define $M^{\prime \prime}$ by

$$
\begin{aligned}
W_{i j}^{\prime \prime} & =\sum_{k=1}^{N} \phi_{i k j}\left(V_{i k}^{\prime} \otimes H_{k j}\right) \\
V_{i j}^{\prime \prime} & =\left\{v \in V_{i j} \mid \phi_{i j k}(v \otimes h) \in W_{i k}^{\prime \prime} \text { for all } k \text { and for all } h \in H_{j k}\right\} .
\end{aligned}
$$

It is immediately clear that these subspaces get respected by the maps $\phi_{i k l}$, and because of the relations $I_{1}^{\prime}$ they are also respected by the maps $A_{k}$ and $B_{k}$ and thus define a subrepresentation. Following the remainder of the elementary proof of [GRT16] Lemma 8.12 we can see that $M^{\prime}$ is subordinated to $M^{\prime \prime}$ and that $M^{\prime \prime}$ is tight.

Putting the results obtained so far together, we arrive at the following criterion for semistability of a representation.

Lemma 3.3.15. Let $M$ denote a representation of $Q\left(Q^{\prime}\right)$ of dimension vector $d$ which satisfies the relations $I_{1}^{\prime}$. Then $M$ is semistable if and only if

$$
\mu^{\prime}\left(M^{\prime}\right) \leq \mu^{\prime}(M)
$$

for all tight non-degenerate subrepresentations $M^{\prime} \subset M$. 
Proof. This works just as [GRT16] Lemma 8.13: Using Lemma 3.3.11 it suffices to check the claimed inequality for non-degenerate subrepresentations. Now combining Lemma 3.3.14 with Lemma 3.3.13 gives the result.

We need to compare subrepresentations and quiver subsheaves. The key construction for this is given by the following Lemma.

Lemma 3.3.16. Let $\mathcal{E}$ denote an $(n, \underline{L})$-regular quiver sheaf of topological type $\tau$. Given a subrepresentation $M^{\prime} \subset M=\operatorname{Hom}(T, \mathcal{E})$ we consider the subsheaves

$$
\mathcal{E}_{i}^{\prime}=\sum_{j=1}^{N}\left(\mathcal{E}_{i}\right)_{j}^{\prime},
$$

where $\left(\mathcal{E}_{i}\right)_{j}^{\prime}=\mathrm{ev}_{j}\left(V_{i j}^{\prime} \otimes L_{j}^{-n}\right)$ is given as the image of the evaluation. These subsheaves are respected by the morphisms $\mathcal{E}_{\alpha}: \mathcal{E}_{i} \rightarrow \mathcal{E}_{j}$ and hence form a quiver subsheaf $\mathcal{E}^{\prime}=\mathcal{E}^{\prime}\left(M^{\prime}\right) \subset \mathcal{E}$.

Proof. Choose any arrow $\alpha: a \rightarrow b$ in $Q$. The induced maps

$$
H^{0}\left(\mathcal{E}_{\alpha} \otimes L_{j}^{-n}\right): H^{0}\left(\mathcal{E}_{a} \otimes L_{j}^{-n}\right) \rightarrow H^{0}\left(\mathcal{E}_{b} \otimes L_{j}^{-n}\right)
$$

map $V_{a j}^{\prime}$ to $V_{b j}^{\prime}$ because $M^{\prime}$ is a subrepresentation by assumption. Hence the diagram

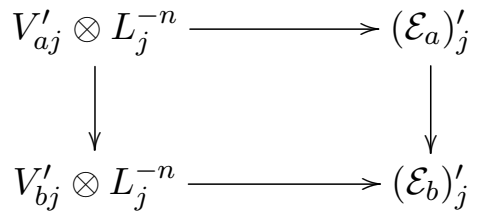

commutes because morphisms of sheaves commute with restriction. Summing up over $j$ gives the result.

Proposition 3.3.17. Suppose that $\mathcal{E}$ is an $(n, \underline{L})$-regular quiver sheaf of topological type $\tau$ and $M^{\prime} \subset M=\operatorname{Hom}(T, \mathcal{E})$ is a subrepresentation. Consider the quiver subsheaf

$$
\mathcal{E}^{\prime} \subset \mathcal{E}
$$

as in Lemma 3.3.16. Then

$$
M^{\prime} \preceq \operatorname{Hom}\left(T, \mathcal{E}^{\prime}\right) .
$$

If $M^{\prime}$ is tight and non-degenerate $M^{\prime}$ satisfies the equality

$$
\mu^{\prime}\left(M^{\prime}\right)=\mu^{\prime}\left(\operatorname{Hom}\left(T, \mathcal{E}^{\prime}\right)\right) .
$$

Proof. The proof of [GRT16] Proposition 8.14 applies to each vertex. This is sufficient to obtain a quiver sheaf version.

This remark also applies to the case of a tight subrepresentation, as the proof of [GRT16] shows that $\operatorname{Hom}\left(T, \mathcal{E}^{\prime}\right)$ is non-degenerate and we can apply Lemma 3.3.13. 
Lemma 3.3.18. Let $\mathcal{E}$ denote an $(n, \underline{L})$-regular quiver sheaf of topological type $\tau$. Then the following assertions are equivalent.

(1) $\operatorname{Hom}(T, \mathcal{E})$ is semistable.

(2) For all quiver subsheaves $\mathcal{F} \subset \mathcal{E}$ we have

$$
\sum_{i \in Q_{0}} \sum_{j=1}^{N} h^{0}\left(\mathcal{F}_{i} \otimes L_{j}^{n}\right) P_{\mathcal{E}}^{\sigma}(m) \leq \sum_{i \in Q_{0}} \sum_{j=1}^{N} \sigma_{i j} h^{0}\left(\mathcal{F}_{i} \otimes L_{j}^{m}\right) P_{\mathcal{E}}^{\sigma}(n) .
$$

(3) The above inequality holds for all quiver subsheaves $\mathcal{E}^{\prime} \subset \mathcal{E}$ of the form as in Lemma 3.3.16 for subrepresentations $M^{\prime} \subset \operatorname{Hom}(T, \mathcal{E})$.

Proof. The proof of [GRT16] Lemma 8.15 applies:

The inequality in the second assertions is equivalent to $\theta(\operatorname{Hom}(T, \mathcal{F})) \leq 0$ after unwrapping the definitions. By Proposition 3.3.17 it suffices to check this inequality on subrepresentations of the form $\operatorname{Hom}\left(T, \mathcal{E}^{\prime}\right)$.

Finally, we are ready to prove that semistability is respected by the embedding functor.

TheOREM 3.3.19. Let $\mathcal{E}$ denote a quiver sheaf of topological type $\tau$ which satisfies the relations $I$. Then $\mathcal{E}$ is semistable if and only if $\mathcal{E}$ is pure, $(p, \underline{L})-$ regular and $\operatorname{Hom}(T, \mathcal{E})$ is semistable.

Proof. With the appropriate modifications this can be proven in the same way as the case of sheaves ([GRT16] Theorem 8.16) by combining the preceding results.

\subsection{3. $S$-equivalence under embedding.}

In this subsection, we strengthen the comparison result established in the preceding subsection. For this to work, we need to get rid of degenerate subrepresentations. One way to ensure this is to assume that $\sigma$ is positive, i.e. all its entries are strictly positive. We remind ourselves that we still assume $\sigma$ to be bounded given a set of relations $I$, and semistable quiver sheaves which satisfy these relations to be $(p, \underline{L})$-regular.

Now that we exclude degenerate subrepresentations of $\operatorname{Hom}(T, \mathcal{E})$, the only remaining destabilizing subrepresentations are given by destabilizing quiver subsheaves.

LEMma 3.3.20. Let $\mathcal{E}$ denote a semistable quiver sheaf which satisfies the relations I for a positive stability parameter $\sigma$. Any destabilizing quiver subsheaf $\mathcal{F} \subset \mathcal{E}$ is $(p, \underline{L})$-regular and $\operatorname{Hom}(T, \mathcal{F}) \subset \operatorname{Hom}(T, \mathcal{E})$ is destabilizing as well.

Proof. By Lemma $3.3 .3, \mathcal{E}^{\prime}$ is $(p, \underline{L})$-regular since it is a direct summand

$$
\mathcal{E}^{\prime} \subset \mathcal{E}^{\prime} \oplus \mathcal{E} / \mathcal{E}^{\prime}
$$


of a semistable quiver sheaf of topological type $\tau$ satisfying the relations $I$, which is thus $(p, \underline{L})$-regular by our assumption. In particular, it is $(m, \underline{L})-$ regular and $(n, \underline{L})$-regular as well. This yields the equations

$$
\begin{aligned}
\mu^{\prime}(\operatorname{Hom}(T, \mathcal{F})) & =\frac{\sum_{i j} \sigma_{i j} h^{0}\left(\mathcal{F}_{i} \otimes L_{j}^{n}\right)}{\sum_{i, j} \sigma_{i j} h^{0}\left(\mathcal{F}_{i} \otimes L_{j}^{m}\right)}=\frac{P_{\mathcal{F}}^{\sigma}(n)}{P_{\mathcal{F}}^{\sigma}(m)}=\frac{p_{\mathcal{F}}^{\sigma}(n)}{p_{\mathcal{F}}^{\sigma}(m)} \\
& =\frac{p_{\mathcal{E}}^{\sigma}(n)}{p_{\mathcal{E}}^{\sigma}(m)}=\mu^{\prime}(\operatorname{Hom}(T, \mathcal{E})),
\end{aligned}
$$

which proves that $\operatorname{Hom}(T, \mathcal{F})$ is destabilizing.

Lemma 3.3.21. Suppose that $M$ is a $\theta$-semistable representation, where $\theta$ is defined using a positive stability condition $\sigma$. A destabilizing subrepresentation $M^{\prime} \subset M$ is then tight.

Proof. The elementary proof of [GRT16] Lemma 8.18 applies word for word.

We are now ready to prove that subrepresentations of $\operatorname{Hom}(T, \mathcal{E})$ which are destabilizing can be deduced from destabilizing quiver subsheaves.

LEMma 3.3.22. Suppose that $\sigma$ denotes a positive stability condition and let $\mathcal{E}$ be a semistable quiver sheaf of topological type $\tau$ which satisfies the relations $I$. For any destabilizing subrepresentation

$$
M^{\prime} \subset \operatorname{Hom}(T, \mathcal{E})
$$

the quiver subsheaf $\mathcal{E}^{\prime} \subset \mathcal{E}$ given as in Proposition $3.3 .1^{77}$ is either equal to $\mathcal{E}$ or destabilizing as well.

Proof. Consider our version of the Le Potier-Simpson theorem 3.3.4 and Condition 5 on $m$ and $n$ for quiver sheaves. The proof of Lemma 8.19 in [GRT16] then carries over.

We end this subsection with the desired result.

TheOrem 3.3.23. Suppose that $\sigma$ is a positive stability condition. For semistable quiver sheaves $\mathcal{E}$ of topological type $\tau$ which satisfy the relations $I$ the identity

$$
\operatorname{Hom}(T, \operatorname{gr}(\mathcal{E})) \simeq \operatorname{gr}(\operatorname{Hom}(T, \mathcal{E}))
$$

holds. Hence, $\operatorname{Hom}(T, *)$ respects $S$-equivalence and $\mathcal{E}$ is stable if and only if $\operatorname{Hom}(T, \mathcal{E})$ is.

Proof. Essentially, the proof of [GRT16] Theorem 8.20 applies, which we repeat here as a sketch.

Consider a Jordan-Hölder filtration

$$
0=\mathcal{E}^{0} \subsetneq \mathcal{E}^{1} \subsetneq \ldots \subsetneq \mathcal{E}^{l}=\mathcal{E}
$$

in the category of quiver sheaves. That is, the quiver subsheaves $\mathcal{E}^{i}$ are destabilizing and the filtration is maximal with this property. Let $M^{i}=$ $\operatorname{Hom}\left(T, \mathcal{E}^{i}\right)$ and $M=\operatorname{Hom}(T, \mathcal{E})$ denote the image of the filtration under the embedding, so that

$$
0=M^{0} \subsetneq M^{1} \subsetneq \ldots \subsetneq M^{l}=M .
$$


If we assume that this filtration allows a refinement, i.e. $M^{p} \subset M^{\prime} \subset M^{p+1}$ for a destabilizing subrepresentation $M^{\prime} \subset M$, we get that $\mathcal{E}^{p} \subset \mathcal{E}^{\prime} \subset \mathcal{E}^{p+1}$ by an argument as in [GRT16], where $\mathcal{E}^{\prime}$ is given as in Lemma 3.3.16. But this contradicts the maximality of the Jordan-Hölder filtration of $\mathcal{E}$.

The exactness of the embedding functor now implies that it respects $S$ equivalence since

$$
\begin{aligned}
\operatorname{gr}(\operatorname{Hom}(T, \mathcal{E})) & =\bigoplus_{k=1}^{l} \operatorname{Hom}\left(T, \mathcal{E}^{k}\right) / \operatorname{Hom}\left(T, \mathcal{E}^{k-1}\right) \\
& =\operatorname{Hom}\left(T, \bigoplus_{k=1}^{l} \mathcal{E}^{k} / \mathcal{E}^{k-1}\right)=\operatorname{Hom}(T, \operatorname{gr}(\mathcal{E})) .
\end{aligned}
$$

This also implies that $\operatorname{Hom}(T, *)$ can distinguish stability from semistability; because $\mathcal{E}$ is stable if and only if $\operatorname{gr}(\mathcal{E})=\mathcal{E}$ and $\operatorname{Hom}(T, \mathcal{E})$ is stable if and only if $\operatorname{gr}(\operatorname{Hom}(T, \mathcal{E}))=\operatorname{Hom}(T, \mathcal{E})$.

\subsection{Construction of the moduli space}

The actual construction of the moduli space is the final step in our program, which can be summarized as follows. The existence of the tautological family $\mathbb{M}$ on the representation variety gives a subscheme which is the image of the embedding functor, and openness of semistability provides an open subscheme of it. Finally, the GIT quotient of the representation variety descends to this subscheme because stability of representations and quiver sheaves are compatible, and the quotient thus obtained is the moduli space of quiver sheaves.

Suppose that we are given a bounded and positive stability parameter $(\underline{L}, \sigma)$ for some fixed set of relations $I$. We fix integers $m \gg n \gg p \gg 0$ such that the following assertions hold.

(1) All $\sigma$-semistable quiver sheaves which satisfy the relations $I$ are $(p, \underline{L})-$ regular.

(2) The functor $\operatorname{Hom}(T, *)$ is an embedding, i.e. Theorem 3.2.7 holds.

(3) Stability is preserved by $\operatorname{Hom}(T$, *), i.e. Theorem 3.3.19 holds.

(4) $S$-equivalence is preserved as well, i.e. Theorem 3.3.23 holds.

Consider the representation variety

$$
R=R_{d}\left(Q\left(Q^{\prime}\right), H, I^{\prime}(I)\right)
$$

for the twisted quiver of dimension vector $d=d(\tau, m, n)$ and recall that we have a group action of $G=G_{d}$ on it as well as a stability condition $\theta=\theta(d, \sigma)$. The tautological family $\mathbb{M}$ of right- $A$-modules, where $A$ is the path algebra of the twisted quiver with labels and relations, gives us a locally closed subscheme

$$
i: B=R_{\tau}^{[\mathrm{reg}]} \subset R
$$

according to Proposition 3.2.13. In particular, the fibers of the family

$$
i^{*} \mathbb{M} \otimes_{A^{\prime}}^{\prime} T
$$


are $(n, \underline{L})$-regular quiver sheaves of topological type $\tau$. Recall (Proposition 3.2.15) that the moduli functor $\mathcal{M}^{\text {reg }}(X, Q, I)$ of $(n, \underline{L})$-regular quiver sheaves on $X$ is locally isomorphic to the quotient functor $\underline{B} / \underline{G}$. The openness of regularity, stability and semistability (Proposition 3.1.23) provides us with open subschemes

$$
B^{[\mathrm{st}]} \subset B^{[\mathrm{sst}]} \subset B^{[\mathrm{reg}]} \subset B,
$$

where the members of the family $i^{*} \mathbb{M} \otimes_{A^{\prime}}^{\prime} T$ are stable, semistable and $(p, \underline{L})$ regular respectively.

Proposition 3.4.1. There is a local isomorphism of functors

$$
\mathcal{M}_{\tau}^{\text {sst }}(Q, X, I) \simeq \underline{B^{[\mathrm{sst}]}} / \underline{G},
$$

and a similar local isomorphism for the case of stable quiver sheaves.

Proof. We have a natural transformation

$$
\underline{\left.B^{[s s t}\right]} / \underline{G} \rightarrow \mathcal{M}_{\tau}^{\text {sst }}(X, Q, I),
$$

given by pulling back the restriction of the family $i^{*} \mathbb{M} \otimes_{A^{\prime}}^{\prime} T$ to $B^{[s s t]}$. As in the proof of [ACK07] Theorem 6.1, this is the restriction of the natural transformation which induced the local isomorphism of Proposition 3.2.15. The same argument applies to the stable case.

Proposition 3.4.2. There exists a commuting diagram

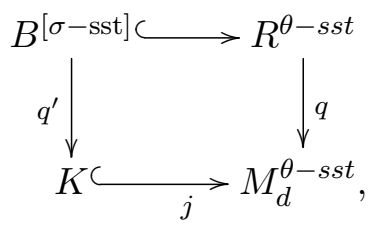

where $M_{d}^{\theta-\text { sst }}=M_{d}^{\theta-\text { sst }}\left(Q\left(Q^{\prime}\right), H, I^{\prime}(I)\right), q$ is the GIT quotient as in Theorem 1.2.21, the restriction $q^{\prime}$ is a good quotient and $j$ is the embedding of a locally closed subscheme.

Proof. Let $Y$ denote $B^{[\text {sst }]}$. By definition, the pullback of the tautological family $\mathbb{M}$ to $Y$ satisfies

$$
i^{*} \mathbb{M} \simeq \operatorname{Hom}^{\prime}(T, \mathbb{E})
$$

for a family $\mathbb{E}$ of $(p, \underline{L})$-regular semistable quiver sheaves of topological type $\tau$ which satisfy the relations $I$.

Consider $Z=\bar{Y} \cap R^{\theta-s s t}$, where $\bar{Y}$ is the closure in $R$. This is a closed and $G^{-}$ invariant subscheme in $R^{\theta-s s t}$. By our assumption that $k$ has characteristic zero, we know that $q$ induces a good quotient on $Z$, and it thus suffices to show that $Y$ lies in $Z$ and that $Y \subset Z$ satisfies the assumptions of Lemma 3.4.3. To see the inclusion, note that for a point $x \in Y$ the fiber

$$
\mathbb{M}_{x} \simeq \operatorname{Hom}(T, \mathcal{E})
$$

is $\theta$-semistable due to Theorem 3.3.19. A similar argument yields the technical condition involving the closed orbits in the closure. Indeed, as a point $x \in Q^{[s s t]}$ corresponds to some representation $M=\operatorname{Hom}(T, \mathcal{E})$ for 
a semistable quiver sheaf $\mathcal{E}$, the closed orbit in the closure of the orbit of $x$ corresponds to the module

$$
\operatorname{gr}(M)=\operatorname{gr}(\operatorname{Hom}(T, \mathcal{E})) \simeq \operatorname{Hom}(T, \operatorname{gr}(\mathcal{E})),
$$

where the latter isomorphism is given by Theorem 3.3.23. Since $\operatorname{gr}(E)$ is again semistable of topological type $\tau$, the closed orbit is also contained in the image of the semistable quiver sheaves under the embedding functor.

We repeat the technical lemma used in the preceding theorem as given in [ACK07], Lemma 6.2.

LEMma 3.4.3. Suppose that the action of a reductive group $G$ on a scheme $Z$ admits a good quotient $\pi: Z \rightarrow Z / / G$. Further, suppose that $Y \subset Z$ denotes an open and $G$-invariant subset such that for each orbit $\mathcal{O} \subset Y$ the unique closed orbit $\mathcal{O}^{\prime}$ contained in the closure $\overline{\mathcal{O}}$ is also contained in $Y$. Then, $\pi$ restricts to a good quotient

$$
\left.\pi\right|_{Y}: Y \rightarrow \pi(Y)
$$

of $Y$, where $\pi(Y) \subset Z / / G$ is open.

Theorem 3.4.4. The scheme $K$ as in Proposition 3.4.2 is the coarse moduli space for semistable quiver sheaves of topological type $\tau$ which satisfy the relations $I$. The closed points of

$$
K=M_{\tau}^{\sigma-s s t}(X, Q, I)
$$

correspond to $S$-equivalence classes of semistable quiver shaves. Furthermore, there exists an open subscheme

$$
M_{\tau}^{\sigma-\mathrm{st}}(X, Q, I) \subset M_{\tau}^{\sigma-\mathrm{sst}}(X, Q, I)
$$

which is the coarse moduli space for stable quiver sheaves of topological type $\tau$. Its closed points correspond to isomorphism classes of stable quiver sheaves.

Proof. Since $q: B^{[s s t]} \rightarrow K$ is a good quotient, the corresponding natural transformation

$$
\underline{q}: \underline{B}\left[{ }^{[\mathrm{sst}]} / \underline{G} \rightarrow \underline{K}\right.
$$

corepresents the quotient functor $\underline{B}^{[s s t]} / \underline{G}$ (compare with the remark to Definition [ACK07], Definition 4.6). But this quotient functor is locally isomorphic to the moduli functor of semistable quiver sheaves by Proposition 3.2.15. Hence $M$ also corepresents this moduli functor (where we use [ACK07], Lemma 4.7).

The closed points of $K$ correspond to closed orbits in $B^{[s s t]}$. These orbits further correspond to $S$-equivalence classes of $A$-modules (consider Proposition 1.2.20), which are of the form $\operatorname{Hom}(T, \mathcal{E})$ for some semistable quiver sheaf $\mathcal{E}$ of topological type $\tau$, as explained in the proof of Proposition 3.4.2. We also know by Theorem 3.3.23 that $S$-equivalence classes of such modules correspond to $S$-equivalence classes of semistable quiver sheaves.

A semistable quiver sheaf $\mathcal{E}$ is stable if and only if the $A$-module $\operatorname{Hom}(T, \mathcal{E})$ is stable. Hence

$$
B^{[\mathrm{st}]}=B^{[\mathrm{sst}]} \cap R^{\theta-\mathrm{st}} .
$$


The geometric quotient of $R^{\theta-s t}$ then restricts to a geometric quotient $B^{[\mathrm{st}]} \rightarrow q\left(B^{[\mathrm{st}]}\right)=K^{\prime} \subset K$, which has open image inside $K$.

Repeating the argument above we see that $K^{\prime}$ corepresents the moduli functor of stable quiver sheaves, and that its closed points correspond to $S_{-}$ equivalence classes of stable quiver sheaves. But $S$-equivalence reduces to isomorphism for stable objects.

COROllary 3.4.5. The moduli space of semistable quiver sheaves satisfying the relations

$$
M_{\tau}^{\text {sst }}(X, Q, I) \subset M_{\tau}^{\text {sst }}(X, Q)
$$

is a closed subscheme of the moduli space without relations, and the same assertion holds for the moduli space of stable quiver sheaves.

Proof. The representation variety with relations $I^{\prime}(I)=I_{1}^{\prime} \cup I_{2}^{\prime}$ is a closed subvariety

$$
R_{d}\left(Q\left(Q^{\prime}\right), H, I^{\prime}(I)\right) \subset R_{d}\left(Q\left(Q^{\prime}\right), H, I_{1}^{\prime}\right),
$$

and the tautological family $\mathbb{M}$ of right- $A$-modules on $R=R_{d}\left(Q\left(Q^{\prime}\right), H, I_{1}^{\prime}\right)$ restricts to a family $\mathbb{M}^{\prime}$ of modules additionally satisfying the relations $I_{2}^{\prime}$ on $R^{\prime}=R_{d}\left(Q\left(Q^{\prime}\right), H, I^{\prime}(I)\right)$. According to Corollary 3.2.14 we thus have

$$
\left(R^{\prime}\right)^{[\mathrm{reg}]}=R^{\prime} \cap R^{[\mathrm{reg}]},
$$

i.e. $\left(R^{\prime}\right)^{[\mathrm{reg}]}$ is a closed subscheme of $R^{[\mathrm{reg}]}$. Further, the property of stability and semistability is insensitive to the question whether relations are imposed, so that the corresponding open subschemes parametrizing stable and semistable quiver sheaves are closed inside the corresponding open subschemes without the relations $I_{2}^{\prime}$. This inclusion is respected by the quotient.

\subsection{Projectivity of the moduli space}

In this section we want to show that the moduli space of quiver sheaves is projective, if the quiver $Q$ does not contain oriented cycles. In face of the similar result for moduli spaces of representations (compare with Theorem 1.2.21) it seems unlikely that one can get rid of this condition. Furthermore, we require that $\sigma$ is a rational stability condition for a crucial technical step of Theorem 3.5.11 below.

Since the moduli space with relations is a closed subscheme of the moduli space without relations (consider Corollary 3.4.5), it is sufficient to show the projectivity for the case without relations, up to the question of boundedness. We consider the case of a smooth and projective scheme $X$.

Theorem 3.5.1. Assume that $Q$ does not contain oriented cycles, and that $\sigma$ is rational. Then, the moduli space of multi-Gieseker semistable quiver sheaves of topological type $\tau$ is projective over $k$.

Proof. Consider the proof of [ACK07] Proposition 6.6, which holds almost word for word in our setting. Nevertheless, we give a brief sketch to point out the relevant references. 
Let $R$ denote a discrete valuation ring over $k$ with field of fractions $K$. The valuative criterion then requires us to construct an extension

$$
x: \operatorname{Spec}(R) \rightarrow M^{\text {sst }}
$$

for any given morphism $x_{0}: \operatorname{Spec}(K) \rightarrow M^{\text {sst }}=M_{\tau}^{\text {sst }}(Q, X)$. The identification of the moduli space with a quotient as in Proposition 3.4.2 allows a lift

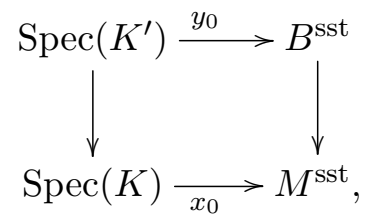

where $K \subset K^{\prime}$ is a suitable finite extension of fields. Denote by $R^{\prime}$ a discrete valuation ring with field of fractions $K^{\prime}$ which dominates $R$.

The remainder of the proof then reduces, modulo borrowing arguments from [ACK07], to showing that a flat family over $\operatorname{Spec}\left(K^{\prime}\right)$ of semistable quiver sheaves of topological type $\tau$ on $X$ extends to a flat family of such quiver sheaves over $\operatorname{Spec}\left(R^{\prime}\right)$.

By Proposition 3.5.4 we can extend the family to a flat one since $Q$ does not contain oriented cycles. Our version of Langton's theorem 3.5.11 then allows to modify the family to obtain a family of semistable quiver sheaves as needed.

REMARK 3.5.2. For the strategy of proof presented here, both the condition that $Q$ contains no cycles, and the condition that $\sigma$ is rational, are essential. Interestingly, for extensions of families of quiver sheaves (as in Subsection 3.5.1), the rationality of $\sigma$ plays no role, while for the proof of Langton's theorem (consider Subsection 3.5.2), we may allow cycles in $Q$.

\subsubsection{Extensions.}

In this subsection we construct extensions of flat families of quiver sheaves from the base $\operatorname{Spec}(K)$ to $\operatorname{Spec}(R)$, where $K$ is the quotient field of a discrete valuation ring $R$. A condition for this to work is that the quiver does not contain oriented cycles.

Let $U \subset X$ denote an open subset of a noetherian scheme over $k$ with inclusion map $i$. It is well-known that coherent sheaves on $U$ can be extended to coherent sheaves on $X$, but we need to ensure that morphisms extend as well. For the case of a quiver without oriented cycles we can show that this even holds for quiver sheaves.

Proposition 3.5.3. Consider a quiver sheaf $\mathcal{E}$ on $U$ over a quiver $Q$ without oriented cycles. There exists a quiver sheaf $\mathcal{E}^{\prime}$ on $X$ such that

$$
\left.\mathcal{E}^{\prime}\right|_{U}=\mathcal{E} \text {. }
$$

This extension is given as a quiver subsheaf of the quasi coherent quiver sheaf $i_{*} \mathcal{E}$. 
Proof. We recall the construction of the extension for a single coherent sheaf $F$ on $U$. As a first step, suppose that $X=\operatorname{Spec}(A)$ is affine. The push forward under the inclusion map $i$ is quasi coherent and hence given by an $A$-module

$$
i_{*} F=\widetilde{M_{F}} .
$$

The module $M_{F}$ can be written $\mathrm{n}$ as a projective limit

$$
M_{F}=\sum_{N} N=\overleftrightarrow{\lim }_{N} N
$$

where the sum and limit are taken over the projective system of finitely generated submodules $N \subset M_{F}$. Both the restriction functor and the tilde functor preserve colimits, which yields

$$
F=\left.i_{*} F\right|_{U}=\left.\varlimsup_{N} \tilde{N}\right|_{U}=\sum_{N} \tilde{N}
$$

But this sum is equal to one of the summands because $F$ is coherent, so that $F=\left.\widetilde{N^{0}}\right|_{U}$. In other words $F^{\prime}=\widetilde{N^{0}}$ is the desired extension.

For the general case take an affine open covering $X=V_{1} \cup \ldots \cup V_{n}$ and use induction on $n$. If an extension $F^{\prime}$ is already given on $X_{n}=V_{1} \cup \ldots \cup V_{n}$, that is $\left.F^{\prime}\right|_{X_{n} \cap U}=\left.F\right|_{X_{n} \cap U}$, we apply the above construction to the open subset $\left(X_{n} \cup U\right) \cap V_{n+1} \subset V_{n+1}$ to obtain an extension to $V_{n+1}$.

To construct the extension of a quiver sheaf we recursively apply a slight variation of this argument. It is easy to see that the same argument for the reduction step to an affine scheme also holds in the quiver sheaf setting; hence assume $X=\operatorname{Spec}(A)$.

If $i \in Q_{0}$ is a source of the quiver, we use the construction to obtain some extension $\mathcal{E}_{i}^{\prime}=\left(N_{i}^{0}\right)^{\sim}$ for a finitely generated submodule $N_{i}^{0} \subset M_{i}=M_{\mathcal{E}_{i}}$. Suppose we have a vertex $j \in Q_{0}$ such that for all its predecessors, i.e. vertices which allow an arrow $\alpha_{k}: i_{k} \rightarrow j$, the extensions are already constructed. That is

$$
\mathcal{E}_{i_{k}}^{\prime}=\left(N_{i_{k}}^{0}\right)^{\sim}
$$

for certain finitely generated submodules $N_{i_{k}}^{0} \subset M_{i_{k}}$. Under the tilde functor the push forward of the morphisms for the arrows corresponds to morphisms of $A$-modules

$$
\left(i_{*} \mathcal{E}_{\alpha_{k}}: i_{*} \mathcal{E}_{i_{k}} \rightarrow i_{*} \mathcal{E}_{j}\right)=\left(f_{k}: M_{i_{k}} \rightarrow M_{j}\right)^{\sim} .
$$

To construct the extension of $\mathcal{E}_{j}$ we do not consider the full system of finitely generated submodules of $M_{j}$ but the subsystem of finitely generated submodules which contain the finitely generated submodule

$$
\sum_{k} f_{k}\left(N_{i_{k}}^{0}\right) \subset M_{j}
$$

Obviously, the sum over this system again has $M_{j}$ as a limit, and the rest of the construction works as above.

The fact that $Q$ does not contain oriented cycles guarantees that this recursion extends the sheaves at all vertices and the morphisms at the arrows in finitely many steps. 
We need the application of this result to the case of families over a discrete valuation ring.

Proposition 3.5.4. Let $R$ denote a discrete valuation ring with field of fractions $K$, and suppose that $Q$ is a quiver without oriented cycles. Any family $\mathcal{E}$ of quiver sheaves over $K$ extends to a flat family of quiver sheaves over $R$.

Proof. An application of Proposition 3.5.3 to the inclusion

$$
U=X \times \operatorname{Spec}(K) \subset X \times \operatorname{Spec}(R)
$$

provides the existence of an extension. It remains to show flatness.

Locally on $\operatorname{Spec}(A) \times \operatorname{Spec}(K)$, the sheaf $\mathcal{E}_{i}$ is given by an $A \otimes K$-module $M_{i}$, and the push forward $i_{*} \mathcal{E}_{i}$ is given by the same vector space equipped with the structure of an $A \otimes R$-module via the canonical map

$$
A \otimes R \rightarrow A \otimes K .
$$

According to Proposition 3.5.3 the extension $\mathcal{E}_{i}^{\prime}$ is given by some finitely generated submodule $N_{i}^{0}$. Because the canonical map is injective, $M$ is torsion-free as an $R$-module, and so $N_{i}^{0}$ is torsion-free as well. This is sufficient to show flatness over the principal ideal domain $R$.

\subsubsection{Langton's theorem.}

This rather technical subsection is devoted to proving a version of Langton's theorem (introduced in [Lan75]) for multi-Gieseker semistable quiver sheaves. Suppose that we have a flat family of quiver sheaves over the spectrum of a discrete valuation ring. The theorem then states that if the fiber over the open point is semistable, the family can be modified over the closed point such that the fiber there becomes semistable as well.

For the proof we first introduce some technicalities, which are not of general interest with respect to the other sections of this thesis. For the proof of the theorem itself, we mostly follow the reasoning of [HL10], Theorem 2.B.1.

Fix a quiver $Q$, a projective and smooth scheme $X$ over $k$, a dimension $d \leq \operatorname{dim}(X)$ and a stability condition $(\underline{L}, \sigma)$ on $Q$.

For a quiver sheaf $\mathcal{E}$ we consider the number

$$
s(\mathcal{E})=\max \left(\operatorname{dim}\left(\mathcal{E}_{i}\right)\right) \in \mathbb{N} .
$$

Following [HL10] Definition 1.6.1, the category $Q-\operatorname{Coh}(X)_{s}$ is given as the full subcategory of the category $Q-\operatorname{Coh}(X)$ of quiver sheaves $\mathcal{E}$ with $s(\mathcal{E}) \leq s$. Clearly,

$$
Q-\operatorname{Coh}(X)_{s} \subset Q-\operatorname{Coh}(X)_{t}
$$

for $s \leq t$ is a full subcategory, which obviously is closed under subobjects, quotients and extensions. Hence it forms a Serre subcategory, and we can consider the quotient category

$$
Q-\operatorname{Coh}(X)_{d, d^{\prime}}=Q-\operatorname{Coh}(X)_{d} / Q-\operatorname{Coh}(X)_{d^{\prime}-1} .
$$


For background on this construction we refer to [Ga90]. The quotient category is again abelian and the canonical functor

$$
Q-\operatorname{Coh}(X)_{d} \rightarrow Q-\operatorname{Coh}(X)_{d, d^{\prime}}
$$

is exact ([Ga90], Lemma III.1). Note that two objects $\mathcal{E}, \mathcal{F}$ in $Q-\operatorname{Coh}(X)_{d, d^{\prime}}$ are isomorphic if and only if there exists an ordinary morphism $\phi: \mathcal{E} \rightarrow \mathcal{F}$ such that kernel and cokernel of $\phi$ are contained in $Q-\operatorname{Coh}(X)_{d^{\prime}-1}$ ([Ga90] Lemma III.4). In this case we say that they are isomorphic in dimension $d^{\prime}$. By additivity of Hilbert polynomials on exact sequences we thus get a welldefined map

$$
P^{\sigma}: Q-\operatorname{Coh}(X)_{d, d^{\prime}} \rightarrow \mathbb{R}[T]_{d, d^{\prime}},
$$

assigning to a quiver sheaf its multi-Hilbert polynomial with respect to the fixed stability condition. Here, $\mathbb{R}[T]_{d, d^{\prime}}$ denotes the ordered vector space of polynomials of degree at most $d$ modulo polynomials of degree at most $d^{\prime}-1$. By

$$
p(\mathcal{E})=p^{\sigma}(\mathcal{E})
$$

we denote the reduced version.

We say that $\mathcal{E}$ is pure in $Q-\operatorname{Coh}(X)_{d, d^{\prime}}$ if $T_{d-1}(\mathcal{E})=T_{d^{\prime}-1}(\mathcal{E})$. The definition of semistability and stability applies to the relative setting, where we replace the multi-Hilbert polynomial by its class in $\mathbb{R}[T]_{d, d^{\prime}}$. This satisfies the properties of a stability condition in the sense of [R97].

REMARK 3.5.5. Clearly, for $d^{\prime}=0$ we recover the definition of semistability in $Q-\operatorname{Coh}(X)$, and for $d^{\prime}=d-1$ we obtain slope semistability. The case $d^{\prime}=d$ is trivial because the reduced Hilbert polynomial is just the monomial $\frac{1}{d !} T^{d}$.

REMARK 3.5.6. Unless emphasized differently, we are concerned with ordinary quiver sheaves in the proof of Theorem 3.5.11. That is, in writing $\mathcal{F} \in Q-\operatorname{Coh}(X)_{d, d^{\prime}}$ we refer to an object in $Q-\operatorname{Coh}(X)_{d, d^{\prime}}$ which is represented by the quiver sheaf $\mathcal{F}$.

By Proposition 1.9 of [R97], there exist maximally destabilizing subobjects for objects in $Q-\operatorname{Coh}(X)_{d, d^{\prime}}$. For technical reasons we want to make sure that these subobjects are represented by saturated quiver subsheaves.

Lemma 3.5.7. The maximally destabilizing subobject

$$
\mathcal{G} \subset \mathcal{F}
$$

of any quiver sheaf $\mathcal{F} \in Q-\operatorname{Coh}(X)_{d, d^{\prime}}$ is represented by an actual quiver subsheaf $\mathcal{G} \subset \mathcal{F}$ which is saturated.

Proof. First consider any representative and the map $i: \mathcal{G} \rightarrow \mathcal{F}$ giving the inclusion. This provides the diagram

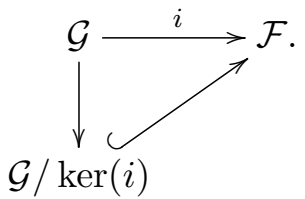


Since $\operatorname{ker}(i)$ is small we may replace $\mathcal{G}$ by $\mathcal{G} / \operatorname{ker}(i)$. In the second step consider the saturation

$$
\mathcal{G} \subset \mathcal{G}_{\text {sat }} \subset \mathcal{F} .
$$

Assuming $\mathcal{G}_{\text {sat }} / \mathcal{G}$ not to be small, we have a strict inclusion $\mathcal{G} \subset \mathcal{G}_{\text {sat }}$ in the quotient category. But this contradicts the maximality of $\mathcal{G}$ because $\mathcal{G}_{\text {sat }}$ has larger multi-Hilbert polynomial (both ordinary and modulo smaller degrees). Hence, $\mathcal{G}$ and $\mathcal{G}_{\text {sat }}$ are isomorphic in $\operatorname{Coh}_{d, d^{\prime}}(X)$.

We need another preparatory lemma.

Lemma 3.5.8. Suppose that $\mathcal{E}$ and $\mathcal{F}$ are quiver sheaves projective scheme $X$ of dimension $n$ such that $s(\mathcal{E}), s(\mathcal{F}) \leq d$ and such that there is an isomorphism $\varphi: \mathcal{E} \rightarrow \mathcal{F}$ in dimension $d-1$. Then the induced morphism

$$
\varphi^{D}: \mathcal{F}^{D} \rightarrow \mathcal{E}^{D}
$$

is an isomorphism.

Proof. It is sufficient to prove the corresponding assertion about sheaves on $X$ in a functorial way. To that end, consider the exact sequence

$$
0 \rightarrow \operatorname{ker}(\varphi) \rightarrow F \rightarrow \operatorname{im}(\varphi) \rightarrow 0,
$$

where by assumption the codimension $c$ of the kernel is greater equal to $n-d$. Consider the induced exact sequence

$$
\begin{aligned}
\operatorname{Ext}^{c-1}\left(\operatorname{ker}(\varphi), \omega_{X}\right) & \rightarrow \operatorname{Ext}^{c}\left(\operatorname{im}(\varphi), \omega_{X}\right) \\
& \rightarrow \operatorname{Ext}^{c}\left(F, \omega_{X}\right) \rightarrow \operatorname{Ext}^{c}\left(\operatorname{ker}(\varphi), \omega_{X}\right) .
\end{aligned}
$$

By [HL10] Proposition 1.1.6 both terms involving the kernel vanish. Hence there is an isomorphism

$$
\varphi^{D}: \operatorname{Ext}^{c}\left(\operatorname{im}(\varphi), \omega_{X}\right) \rightarrow F^{D} .
$$

Applying the same argument to the cokernel sequence shows that

$$
G^{D} \rightarrow \operatorname{Ext}^{c}\left(\operatorname{im}(\varphi), \omega_{X}\right)
$$

is also an isomorphism, which finishes the proof.

Let $X$ denote a projective scheme over an algebraically closed field $k$ of characteristic 0 , and consider a field extension $k \subset K$. Denote by $\mathcal{E}_{K}$ the base change of a quiver sheaf $\mathcal{E}$ on $X$, and by $\underline{L}_{K}$ the base change of the tuple $\underline{L}$. We now show that semistability is preserved by field extensions. This is a variant of [HL10], Theorem 1.3.7.

Proposition 3.5.9. Let $\mathcal{E}$ denote a pure quiver sheaf on a projective scheme $X$ over $k$ which is semistable with respect to some stability condition $(\underline{L}, \sigma)$. Consider a finitely generated field extension $k \subset K$. The pullback $\mathcal{E}_{K}$ is a semistable quiver sheaf on $X_{K}$ with respect to $\left(\underline{L}_{K}, \sigma\right)$.

Proof. We even claim that the Harder-Narasimhan filtrations are compatible in the sense that

$$
\mathrm{HN}_{i}\left(\mathcal{E}_{K}\right)=\mathrm{HN}_{i}(\mathcal{E})_{K}
$$


First note that any morphism $l \rightarrow L$ of fields induces a flat morphism $\operatorname{Spec}(L) \rightarrow \operatorname{Spec}(l)$, so that

$$
h^{0}\left(X_{L}, E_{L}\right)=h^{0}\left(X_{l}, E_{l}\right)
$$

for any coherent sheaf $E_{l}$ on $X_{l}$ (compare with the proof of [Har77] Proposition III.9.3). In particular, this implies that the Hilbert polynomials for quiver sheaves $\mathcal{E}$ on $X=X_{k}$ remain identical in the sense that

$$
p^{(\underline{L}, \sigma)}(\mathcal{E})=p^{\left(\underline{L}_{K}, \sigma\right)}\left(\mathcal{E}_{K}\right) .
$$

Further, the flatness implies that quiver subsheaves and quiver subquotients of $\mathcal{E}$ get mapped to quiver subsheaves and quiver subquotients of $\mathcal{E}_{K}$ respectively.

A first consequence of these remarks is that if $\mathcal{E}_{K}$ is semistable, so is $\mathcal{E}$.

If we can then show that the Harder-Narasimhan filtration of $\mathcal{E}_{K}$ is induced by some filtration $\mathcal{F}^{*}$ of $\mathcal{E}$ in the sense that $\operatorname{HN}_{i}\left(\mathcal{E}_{K}\right)=\left(\mathcal{F}^{i}\right)_{K}$, then $\mathcal{F}^{*}$ satisfies the properties of the Harder-Narasimhan filtration of $\mathcal{E}$.

By induction on the number of generators of the field extension $k \subset K$ we reduce to the case that $K=k(x)$, where $x$ is either transcendental or algebraic and hence separable over $k$ (note that $k$ is perfect).

In the separable case we pass to the normal hull, so that we may assume the extension to be Galois. Thus, $\operatorname{HN}_{i}\left(\mathcal{E}_{K}\right)$ is induced by a quiver subsheaf $\mathcal{F}^{i} \subset \mathcal{E}$ if and only if $\operatorname{HN}_{i}\left(\mathcal{E}_{K}\right)$ is invariant under the induced action of $G=\operatorname{Gal}(K / k)$ on $\mathcal{E}_{K}$. To see this, we note that the corresponding descent question for sheaves is locally a question whether a submodule $N \subset M \otimes_{k} K$ over $R \otimes_{k} K$, where $M$ is a module over some $k$-algebra $R$, is induced by a submodule $N^{\prime} \subset M$ if $N$ is invariant under the induced action of $G$ on $M \otimes_{k} K$. This is true by [Mi15], Proposition 16.7, with $N^{\prime}=M \otimes_{k} k \cap N$. Clearly, these descents are also respected by induced morphisms $f \otimes_{k} K: M_{1} \otimes_{k} K \rightarrow M_{2} \otimes_{k} K$.

In our situation, we can see that for any $g \in G$ the $g * \operatorname{HN}_{i}\left(\mathcal{E}_{K}\right)$ satisfy the properties of the Harder-Narasimhan filtration by applying our initial remarks to the induced morphism $g: \operatorname{Spec}(K) \rightarrow \operatorname{Spec}(K)$. Hence the Harder-Narasimhan filtration is invariant and we are done.

In the case where $x$ is transcendental over $k$, i.e. $K=k(x)$ is the field of rational functions, we can use a similar argument using the relative automorphism group $G=\operatorname{Aut}(K / k)$, once we note that the relative automorphisms

$$
x \mapsto a x
$$

for $a \in k^{*}$ have $k$ as their fixed point field (this follows from the fact that there are no invariant polynomials by using [Muk03] Proposition 6.2). 
Lemma 3.5.10. Let $X \rightarrow S$ denote a morhpism of finite type between noetherian schemes. Suppose that $S_{0} \subset S$ is a closed subscheme defined by a nilpotent ideal sheaf $\mathcal{I} \subset \mathcal{O}_{S}$. Then a quiver sheaf $\mathcal{F}$ on $X$ is flat over $S$ if and only if it is flat over $S_{0}$ and the natural multiplication map

$$
\mathcal{I} \otimes_{S} \mathcal{F} \rightarrow \mathcal{I} F
$$

is an isomorphism.

Proof. This follows from the sheaf version [HL10] Lemma 2.1.3 once we note that the notion of flatness can be checked at each vertex and the natural multiplication map for sheaves extends to quiver sheaves.

We are ready to prove Langton's theorem for families of semistable quiver sheaves.

TheOREM 3.5.11. Let $R$ denote a discrete valuation ring with maximal ideal $\mathfrak{m}=(\pi)$, field of fractions $K$, and residue field $k$.

Let $\mathcal{F}$ be an $R$-flat family of $d$-dimensional quiver sheaves on $X$ such that $\mathcal{F}_{K}=\mathcal{F} \otimes_{R} K$ is semistable in $Q-\operatorname{Coh}\left(X_{K}\right)_{d, d^{\prime}}$ for some $d^{\prime}<d$. Then there exists a quiver subsheaf $\mathcal{E} \subset \mathcal{F}$ such that $\mathcal{E}_{K}=\mathcal{F}_{K}$ and such that $\mathcal{E}_{k}$ is also semistable in $Q-\operatorname{Coh}(X)_{d, d^{\prime}}$.

Proof. We prove the following (stronger) auxiliary statement:

Suppose that for $d^{\prime} \leq \delta<d$ we have that $\mathcal{F}_{k}$ is semistable in $Q-\operatorname{Coh}(X)_{d, \delta+1}$ in addition to the assumptions of the theorem. Then there is a quiver subsheaf $\mathcal{E} \subset \mathcal{F}$ such that $\mathcal{E}_{K}=\mathcal{F}_{K}$ and $\mathcal{E}_{k}$ is semistable in $Q-\operatorname{Coh}(X)_{d, \delta}$.

Assuming that the auxiliary statement is true we obtain the statement of the theorem by induction on $\delta$, where the case $\delta=d-1$ is trivial (compare with Remark 3.5.5). From now on we assume that the auxiliary statement is false. By recursion we will define a descending sequence of quiver sheaves on $X_{R}$

$$
\mathcal{F}=\mathcal{F}^{0} \supset \mathcal{F}^{1} \supset \mathcal{F}^{2} \supset \ldots,
$$

where $\mathcal{F}_{K}^{n}=\mathcal{F}_{K}$ for all $n$. Under the assumption that the auxiliary statement is false each $\mathcal{F}_{k}^{n}$ is unstable in $Q-\operatorname{Coh}(X)_{d, \delta}$.

Suppose that $\mathcal{F}^{n}$ was already defined. Let $\mathcal{K}^{n} \subset \mathcal{F}_{k}^{n}$ be a saturated representative for the maximally destabilizing quiver subsheaf (given by Lemma 3.5.7) and define $\mathcal{G}^{n}=\mathcal{F}_{k}^{n} / \mathcal{K}^{n}$ (note that $\mathcal{G}^{n}$ is pure). Then $\mathcal{F}^{n+1}$ is given as

$$
\mathcal{F}^{n+1}=\operatorname{ker}\left(\mathcal{F}^{n} \rightarrow \mathcal{F}_{k}^{n} \rightarrow \mathcal{G}^{n}\right) .
$$

Note for later use that this implies $\mathcal{K}^{n-1}=\mathcal{F}^{n} / \pi \mathcal{F}^{n-1}$. Because outside of the closed point $\operatorname{Spec}(k)$, that is on $X_{K}$, every section gets mapped to zero we have $\mathcal{F}_{K}^{n+1}=\mathcal{F}_{K}^{n}$.

There is an obvious exact sequence

$$
(S 1): 0 \rightarrow \mathcal{K}^{n} \rightarrow \mathcal{F}_{k}^{n} \rightarrow \mathcal{G}^{n} \rightarrow 0 .
$$

Furthermore, we may now construct a second exact sequence

$$
(S 2): 0 \rightarrow \mathcal{G}^{n} \rightarrow \mathcal{F}_{k}^{n+1} \rightarrow \mathcal{K}^{n} \rightarrow 0 .
$$

Note that using induction these two exact sequences imply

$$
(E Q 1): P^{\sigma}\left(\mathcal{F}_{k}^{n}\right)=P^{\sigma}\left(\mathcal{F}_{k}\right) \in \mathbb{R}[T] .
$$


To construct the second sequence first note that $\mathcal{F}_{k}^{n}=\mathcal{F}^{n} / \pi \mathcal{F}^{n}$, where we denote the projection map as

$$
q: \mathcal{F}^{n} \rightarrow \mathcal{F}_{k}^{n}=\mathcal{F}^{n} / \pi \mathcal{F}^{n} .
$$

By construction of $\mathcal{F}^{n+1}$ and the universal property of the kernel we have an induced map $q_{0}: \mathcal{F}^{n+1} \rightarrow \mathcal{K}^{n}$. Note that $\pi \mathcal{F}^{n} \subset \mathcal{F}^{n+1}$, and $\pi \mathcal{F}^{n}$ clearly gets annihilated by $q$. Hence we obtain an induced morphism

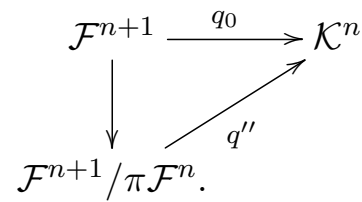

Further note that $\pi \mathcal{F}^{n+1} \subset \pi \mathcal{F}^{n}$, so that $q^{\prime \prime}$ induces a map

$$
q^{\prime}: \mathcal{F}_{k}^{n+1}=\mathcal{F}^{n+1} / \pi \mathcal{F}^{n+1} \rightarrow \mathcal{K}^{n} .
$$

The kernel of this map consists of (classes of) sections of $\mathcal{F}^{n+1}$ which get annihilated by $q_{0}$. Hence

$$
\operatorname{ker}\left(q^{\prime}\right)=\operatorname{ker}\left(q_{0}\right) / \pi \mathcal{F}^{n+1}=\pi \mathcal{F}^{n} / \pi \mathcal{F}^{n+1} .
$$

By flatness, we further know that $\pi \mathcal{F}^{n} \simeq \mathcal{F}^{n}$ and $\pi \mathcal{F}^{n+1} \simeq \mathcal{F}^{n+1}$. Since the maps used for the construction of $\mathcal{F}^{n+1}$ are surjective we also have $\mathcal{G}^{n} \simeq \mathcal{F}^{n} / \mathcal{F}^{n+1}$. This yields $\operatorname{ker}\left(q^{\prime}\right) \simeq \mathcal{G}^{n}$.

To finish the construction of $(S 2)$ observe that the surjectivity of $q^{\prime}$ is inherited from $q_{0}$.

We define $\mathcal{C}^{n}=\mathcal{G}^{n} \cap \mathcal{K}^{n+1}$ considered as quiver subsheaves of $\mathcal{F}_{k}^{n+1}$ via the exact sequences above. Then the exact sequence $(S 2)$ and the obvious inclusions $\mathcal{C}^{n} \subset \mathcal{G}^{n}$ and $\mathcal{C}^{n} \subset \mathcal{K}^{n+1}$ induce a map $\mathcal{K}^{n+1} / \mathcal{C}^{n} \rightarrow \mathcal{K}^{n}$

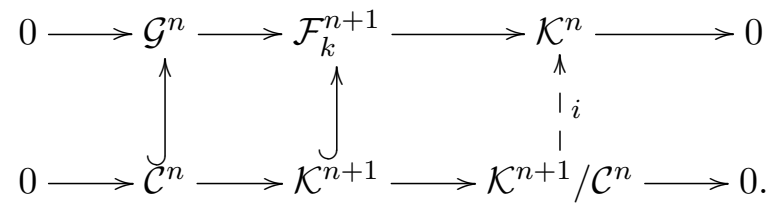

A proof by diagram chasing shows that $i$ is an inclusion (note that $\mathcal{C}^{n}$ is a pullback). In a similar fashion, using sequence $(S 1)$, we get a monomorphism $\mathcal{G}^{n} / \mathcal{C}^{n} \rightarrow \mathcal{G}^{n+1}$.

Assuming that $\mathcal{C}^{n}$ is not isomorphic to zero in $Q-\operatorname{Coh}\left(X_{k}\right)_{d, \delta}$ yields a contradiction as follows. In case $\mathcal{C}^{n}=\mathcal{K}^{n+1}$ we get the inequalities

$$
(I E 1): p^{\sigma}\left(\mathcal{K}^{n+1}\right)=p^{\sigma}\left(\mathcal{C}^{n}\right) \leq p_{\max }^{\sigma}\left(\mathcal{G}^{n}\right)<p^{\sigma}\left(\mathcal{K}^{n}\right) \in \mathbb{R}[T]_{d, \delta} .
$$

The rightmost inequality holds because $p_{\max }^{\sigma}\left(\mathcal{G}^{n}\right)$ and $p^{\sigma}\left(\mathcal{K}^{n}\right)$ are the second and first Harder-Narasimhan weights of $\mathcal{F}_{k}^{n}$ respectively. In case $\mathcal{C}^{n} \neq \mathcal{K}^{n+1}$ we have the inequalities

$$
(I E 2): p^{\sigma}\left(\mathcal{C}^{n}\right)<p^{\sigma}\left(\mathcal{K}^{n+1}\right)<p^{\sigma}\left(\mathcal{K}^{n+1} / \mathcal{C}^{n}\right) \leq p^{\sigma}\left(\mathcal{K}^{n}\right) \in \mathbb{R}[T]_{d, \delta} .
$$

The first and last inequalities hold by the defining property of a destabilizing subobject. Note that the first inequality is strict because of the assumption, and the latter inequality also uses the inclusion constructed above. The 
inequality in the middle is implied by the first one using the obvious exact sequence.

In any case we have

$$
(I E 3): p^{\sigma}\left(\mathcal{K}^{n+1}\right) \leq p^{\sigma}\left(\mathcal{K}^{n}\right) \in \mathbb{R}[T]_{d, \delta} .
$$

If $\mathcal{C}^{n}$ is not isomorphic to zero this holds by $(I E 1)$ and $(I E 2)$. And if $\mathcal{C}^{n} \simeq 0$ this holds because we have the inclusion $\mathcal{K}^{n+1}=\mathcal{K}^{n+1} / \mathcal{C}^{n} \subset \mathcal{K}^{n}$.

Since we assume $\mathcal{F}_{k}$ to be semistable in $Q-\operatorname{Coh}(X)_{d, \delta+1}$ we have that $p^{\sigma}\left(\mathcal{K}^{n}\right) \leq p^{\sigma}\left(\mathcal{F}_{k}^{n}\right) \in \mathbb{R}[T]_{d, \delta+1}$. But strict inequality would also imply

$$
p^{\sigma}\left(\mathcal{K}^{n}\right)<p^{\sigma}\left(\mathcal{F}_{k}^{n}\right) \in \mathbb{R}[T]_{d, \delta},
$$

contradicting the fact that $\mathcal{F}_{k}^{n}$ is not semistable. Together with (EQ1) we thus arrive at

$$
(E Q 2): p^{\sigma}\left(\mathcal{K}^{n}\right)=p^{\sigma}\left(\mathcal{F}_{k}\right) \in \mathbb{R}[T]_{d, \delta+1} .
$$

Hence

$$
p^{\sigma}\left(\mathcal{K}^{n}\right)-p^{\sigma}\left(\mathcal{F}_{k}\right)=\beta_{n} T^{\delta} \in \mathbb{R}[T]_{d, \delta}
$$

for some $\beta_{n} \in \mathbb{R}$. We need some properties of the sequence $\beta_{n} \in \mathbb{R}$.

Because $p^{\sigma}\left(\mathcal{K}^{n}\right)>p^{\sigma}\left(\mathcal{F}_{k}^{n}\right)=p^{\sigma}\left(\mathcal{F}_{k}\right) \in \mathbb{R}[T]_{d, \delta}$, which holds by unstability of $\mathcal{F}_{k}^{n}$ and $(E Q 1)$, the $\beta_{n}$ are strictly positive, and by (IE3) their sequence is decreasing. Finally, the possible values for $\beta_{n}$ are contained in discrete set (consider Lemma 3.5.12). Hence, $\beta_{n}$ must become stationary for $n \gg 0$, and without loss of generality we restrict ourselves to such $n$ in the following.

This implies $p^{\sigma}\left(\mathcal{K}^{n}\right)=p^{\sigma}\left(\mathcal{K}^{n+1}\right)$ in $\mathbb{R}[T]_{d, \delta}$, and because this contradicts both $(I E 1)$ and (IE2) it implies furthermore that

$$
\mathcal{G}^{n} \cap \mathcal{K}^{n+1}=\mathcal{C}^{n} \simeq 0 .
$$

Observe that $\mathcal{C}^{n}$ is a quiver subsheaf of a pure quiver sheaf of dimension $d$ $\left(\mathcal{G}^{n}\right.$ or $\left.\mathcal{K}^{n+1}\right)$. But since it can not have dimension $d$ it must equal zero, so that there are inclusions $i: \mathcal{K}^{n+1} \rightarrow \mathcal{K}^{n}$ as well as $j: \mathcal{G}^{n} \rightarrow \mathcal{G}^{n+1}$ in $Q-\operatorname{Coh}\left(X_{k}\right)_{d, \delta}$.

Note that $P^{\sigma}\left(\mathcal{K}^{n}\right)=P^{\sigma}\left(\mathcal{K}^{n+1}\right) \in \mathbb{R}[T]_{d, \delta}$ as well for large enough $n$ because the rank of the $\mathcal{K}^{n}$ can not descend forever. Combined with $(E Q 1)$ this gives

$$
(E Q 3): P^{\sigma}\left(\mathcal{G}^{n}\right)=P^{\sigma}\left(\mathcal{G}^{n+1}\right) \in \mathbb{R}[T]_{d, \delta} .
$$

Since $\mathcal{G}^{n}$ is pure the kernel of $j$ is either zero or of dimension $d$. Clearly the latter is absurd, and hence there are actual inclusions $j: \mathcal{G}^{n} \subset \mathcal{G}^{n+1}$. Thus we get exact sequences

$$
0 \rightarrow \mathcal{G}^{n} \rightarrow \mathcal{G}^{n+1} \rightarrow \mathcal{G}^{n} / \mathcal{G}^{n+1} \rightarrow 0,
$$

where $(E Q 3)$ implies that the rightmost term is isomorphic to zero in the category $Q-\operatorname{Coh}\left(X_{k}\right)_{d, \delta}$. So the $\mathcal{G}^{n}$ are isomorphic in dimension $\delta$ and thus in particular in dimension $d-1$. By Lemma 3.5.8 their reflexive hulls $\left(\mathcal{G}^{n}\right)^{D D}$ are all isomorphic, so that the sequence

$$
\mathcal{G}^{0} \subset \mathcal{G}^{1} \subset \mathcal{G}^{2} \subset \ldots
$$

is an increasing sequence of quiver subsheaves of the fixed quiver sheaf which is given as this reflexive hull, and must thus become stationary. Again we 
restrict to the case where $n \gg 0$ is such that this is the case and set $\mathcal{G}=\mathcal{G}^{n}$. We can see that this implies that the sequences $(S 1)$ and $(S 2)$ split as follows. Consider the diagram

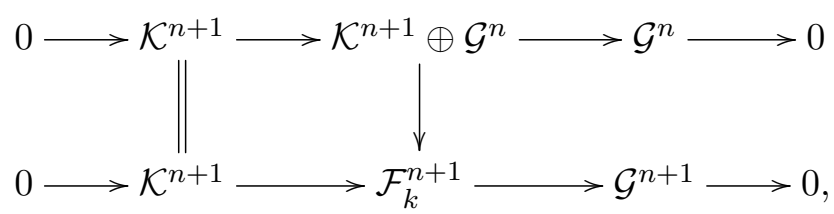

where the first row is given by $(S 1)$ and the middle morphism as the sum of the injective morphisms in $(S 1)$ and $(S 2)$. Thus there is an induced morphism $\mathcal{G}^{n} \rightarrow \mathcal{G}^{n+1}$. By construction, this is exactly the inclusion morphism $\mathcal{G}^{n} \subset \mathcal{G}^{n+1}$, which turned out to be an isomorphism, so that

$$
\mathcal{F}_{k}^{n+1} \simeq \mathcal{K}^{n+1} \oplus \mathcal{G}^{n} \simeq \mathcal{K} \oplus \mathcal{G}
$$

for all $n$ large enough.

Define $\mathcal{E}^{n}=\mathcal{F} / \mathcal{F}^{n}$. Because $\pi \mathcal{F}^{n} \subset \mathcal{F}^{n+1}$, and so by induction $\pi^{n} \mathcal{F} \subset \mathcal{F}^{n}$, there is a well-defined quotient map

$$
\mathcal{F} / \pi^{n} \mathcal{F} \rightarrow \mathcal{F} / \mathcal{F}^{n}=\mathcal{E}^{n} .
$$

By the local flatness criterion for quiver sheaves 3.5.10 we thus know that $\mathcal{E}^{n}$ is flat over $R / \pi^{n}$.

Next, we want to show $\mathcal{E}_{k}^{n} \simeq \mathcal{G}$, which gives us the topological type of $\mathcal{E}^{n}$. Using Noether's isomorphism theorem we obtain

$$
\begin{aligned}
\mathcal{E}_{k}^{n} & =\left(\mathcal{F} / \mathcal{F}^{n}\right) /\left(\pi\left(\mathcal{F} / \mathcal{F}^{n}\right)\right)=\left(\mathcal{F} / \mathcal{F}^{n}\right) /\left(\left(\pi \mathcal{F}+\mathcal{F}^{n}\right) / \mathcal{F}^{n}\right) \simeq \mathcal{F} /\left(\pi \mathcal{F}+\mathcal{F}^{n}\right) \\
& \simeq(\mathcal{F} / \pi \mathcal{F}) /\left(\left(\mathcal{F}^{n}+\pi \mathcal{F}\right) / \pi \mathcal{F}\right)=\mathcal{F}_{k} / \operatorname{im}(\alpha),
\end{aligned}
$$

where $\alpha$ is the composition of the morphisms

$$
\alpha_{n}: \mathcal{F}_{k}^{n} \rightarrow \mathcal{F}_{k}^{n-1}, f+\pi \mathcal{F}^{n} \mapsto f+\pi \mathcal{F}^{n-1} .
$$

Note that

$$
\begin{aligned}
\operatorname{ker}\left(\alpha_{n}\right) & =\pi \mathcal{F}^{n-1} / \pi \mathcal{F}^{n} \simeq \mathcal{F}^{n-1} / \mathcal{F}^{n} \simeq \mathcal{G}^{n-1} \\
\operatorname{im}\left(\alpha_{n}\right) & =\mathcal{F}^{n} / \pi \mathcal{F}^{n-1}=\mathcal{K}^{n-1},
\end{aligned}
$$

which implies that we get decompositions

$$
\mathcal{F}_{k}^{n}=\mathcal{K}^{n} \oplus \mathcal{G}^{n-1}=\operatorname{im}\left(\alpha_{n+1}\right) \oplus \operatorname{ker}\left(\alpha_{n}\right) .
$$

Hence $\operatorname{im}(\alpha)=\operatorname{im}\left(\alpha_{1}\right)=\mathcal{K}^{0}$ and $\mathcal{E}_{k}^{n}=\mathcal{G}^{0} \simeq \mathcal{G}$ as desired.

Summarizing these results we have shown that $\mathcal{E}^{n}$ is a quotient

$$
\mathcal{F}_{R_{n}} \rightarrow \mathcal{E}^{n} \rightarrow 0
$$


which is flat over $R_{n}=R / \pi^{n}$. This corresponds to a morphism $\phi$ over $\operatorname{Spec}(R)$ which fits into a diagram

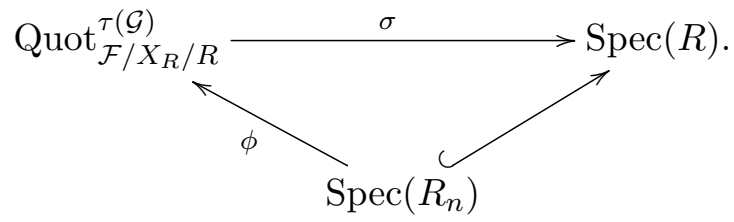

Hence the image of $\sigma$ contains the closed subschemes $\operatorname{Spec}\left(R_{n}\right)$ of $\operatorname{Spec}(R)$ for all $n$, which is only possible if $\sigma$ is surjective.

The morphism $\operatorname{Spec}(K) \rightarrow \operatorname{Spec}(R)$ corresponds to a point $y \in \operatorname{Spec}(R)$ such that $k(y) \subset K$. By surjectivity of $\sigma$ we can find an inverse image $x \in \operatorname{Quot}_{\mathcal{F} / X_{R} / R}^{\tau(\mathcal{G})}(\mathcal{F}, \tau(\mathcal{G}))$. Let $K^{\prime}$ denote the common extension of the induced field extension $k(x) \subset k(y)$ and the extension $k(x) \subset K$, so that

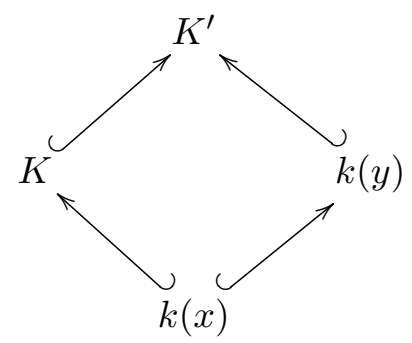

Reversing the correspondences used above the extension $k(y) \subset K^{\prime}$ gives a morphism $\operatorname{Spec}\left(K^{\prime}\right) \rightarrow \operatorname{Quot}_{\mathcal{F} / X_{R} / R}^{\tau(\mathcal{G})} \operatorname{over} \operatorname{Spec}(R)$ and hence a quotient

$$
\mathcal{F}_{K^{\prime}} \rightarrow \mathcal{U} \rightarrow 0
$$

with topological type $\tau(\mathcal{G})$. By Proposition 3.5.9 we know that $\mathcal{F}_{K^{\prime}}$ is semistable. But by our assumptions, the Hilbert polynomials satisfy the inequality

$$
p^{\sigma}(\mathcal{U})=p^{\sigma}(\mathcal{G})>p^{\sigma}\left(\mathcal{F}_{k}^{n}\right)=p^{\sigma}\left(\mathcal{F}_{K^{\prime}}\right) .
$$

This is a contradiction.

The fact that the stability condition $\sigma$ is rational is crucial for the validity of the proof of Theorem 3.5.11. Because of its significance, we state the relevant step as a separate lemma.

LEMMA 3.5.12. With notation as in the proof of Theorem 3.5.11, the numbers $\beta_{n}$ such that

$$
p^{\sigma}\left(\mathcal{K}^{n}\right)-p^{\sigma}\left(\mathcal{F}_{k}\right)=\beta_{n} T^{\delta}
$$

are contained in a discrete set, if $\sigma$ is rational.

Proof. Since the polynomial $p^{\sigma}\left(\mathcal{F}_{k}\right)$ is independent of $n$, it remains to show that the coefficients of $p^{\sigma}\left(\mathcal{K}^{n}\right)$ take values in a discrete set. Such a coefficient is given as

$$
\frac{\sum_{i \in Q_{0}} \sum_{j=1}^{N} \sigma_{i j} \alpha_{\delta}^{L_{j}}\left(\mathcal{K}_{i}^{n}\right)}{\sum_{i \in Q_{0}} \sum_{j=1}^{N} \sigma_{i j} \alpha_{d}^{L_{j}}\left(\mathcal{K}_{i}^{n}\right)} .
$$


By construction, $\mathcal{K}^{n}$ is a quiver subsheaf of $\mathcal{F}_{k}^{n}$, so that each $\alpha_{d}^{L_{j}}\left(\mathcal{K}_{i}^{n}\right)$ is an integer between 1 and $\alpha_{d}^{L_{j}}\left(\mathcal{K}_{i}^{n}\right)$. The upper bound is independent of $n$ because $\mathcal{F}_{K}^{n}=\mathcal{F}_{K}^{n+1}$ and $\mathcal{F}^{n}$ is flat, so that there are only finitely many possible values for the denominator.

For $\delta<d$ it is well-known that the coefficients $\alpha_{\delta}^{L_{j}}\left(\mathcal{K}_{i}^{n}\right)$ take value in some lattice $(1 / r !) \mathbb{Z}$. The numerator thus takes values in a set of the form

$$
\mathbb{Z} a_{1}+\ldots+\mathbb{Z} a_{r}
$$

for finitely many rational numbers $a_{r}$. By factoring out the denominators of the $a_{i}$, we see that this set is contained in a cyclic subgroup $\mathbb{Z} \alpha \subset \mathbb{R}$, and is thus discrete.

REMARK 3.5.13. If $\alpha \in \mathbb{R}$ is irrational, the set

$$
\mathbb{Z}+\mathbb{Z} \alpha \subset \mathbb{R}
$$

is dense. Hence, for $\sigma$ which are not rational, the proof of Lemma 3.5.12, and hence the proof of Theorem 3.5.11, is no longer valid.

\subsection{Variation of stability conditions}

In this section we study the question of the dependence of the moduli spaces on the stability parameter $\sigma$. We consider some given (possibly empty) set of relations $I$.

\subsubsection{Walls and chambers.}

Fix a natural number $p$, a topological type $\tau$, a dimension $d$ and a tuple $\underline{L}$ of ample line bundles on some projective scheme $X$. Furthermore, consider a fixed subset

$$
\Sigma \subset\left(\mathbb{R}^{Q_{0} \times N}\right)_{+}
$$

which is separated from the boundary of $\left(\mathbb{R}^{Q_{0} \times N}\right)_{+}$. That is, we require the existence of a closed polyhedral cone

$$
\Sigma \subset \Sigma^{\prime} \subset \mathbb{R}^{Q_{0} \times N}
$$

which, except for the origin, is contained in $\left(\mathbb{R}^{Q_{0} \times N}\right)_{+}$. We recall that a chamber structure on $\Sigma^{\prime}$, in the sense of [GRT16] Definition 4.1, is a collection of real hypersurfaces

$$
W=\left(W_{i}, i \in J\right) .
$$

The chambers are the maximal connected subsets $C \subset \mathbb{R}^{n}$ such that for each wall $W_{i}$ either $C \subset W_{i}$ or $C \cap W_{i}=\emptyset$ holds. Note that, by definition, the chambers are connected components of real semialgebraic sets, and so are path-connected as well (since they even admit triangulations according to [Lo64]). 
We consider the family $S$ of quiver subsheaves $\mathcal{F} \subset \mathcal{E}$ which satisfy the conditions

(1) $\mathcal{E}$ is pure of dimension $d,(p, \underline{L})$-regular, of topological type $\tau$, and satisfies the relations $I$

(2) $\mathcal{F} \subset \mathcal{E}$ is saturated,

(3) $\widehat{\mu}^{\sigma}(\mathcal{F}) \geq \widehat{\mu}^{\sigma}(\mathcal{E})$ for some $\sigma \in \Sigma^{\prime}$.

We need to ensure that this family is bounded, and the proof of this fact employs the properties of the auxiliary cone $\Sigma^{\prime}$.

Lemma 3.6.1. The family $S$ is bounded.

Proof. Since the topological type $\tau$ is fixed, the expression

$$
\widehat{\mu}^{\sigma}(\mathcal{E})=\frac{\sum_{i \in Q_{0}} \sum_{j=1}^{N} \sigma_{i j} \alpha_{d-1}^{L_{j}}\left(\mathcal{E}_{i}\right)}{\sum_{i \in Q_{0}} \sum_{j=1}^{N} \sigma_{i j} \alpha_{d}^{L_{j}}\left(\mathcal{E}_{i}\right)}
$$

does only depend on $\sigma \in \Sigma^{\prime}$. By an argument as in the proof of the Grothendieck lemma 3.1.21, this is bounded from below as a function in $\sigma$. Hence, $S$ is bounded by the Grothendieck lemma.

To each quiver sheaf $\mathcal{F} \in S$, and each integer $0 \leq e \leq d-1$, we can associate a wall. It is defined by the equation

$$
W_{e, \mathcal{F}}: \sum_{i, k \in Q_{0}} \sum_{j, l=1}^{N} \sigma_{i j} \sigma_{k l}\left(\alpha_{e}^{L_{j}}\left(\mathcal{F}_{i}\right) \alpha_{d}^{L_{l}}\left(\mathcal{E}_{k}\right)-\alpha_{e}^{L_{j}}\left(\mathcal{E}_{i}\right) \alpha_{d}^{L_{l}}\left(\mathcal{F}_{k}\right)\right)=0 .
$$

The chamber structure we consider is given by the equations which are nontrivial, i.e. which carve out neither the empty set nor the whole of $\Sigma^{\prime}$.

Note that $\sigma$ is contained in the wall $W_{e, \mathcal{F}}$ if $p_{\mathcal{E}}^{\sigma}$ and $p_{\mathcal{F}}^{\sigma}$ have the same coefficient in degree $e$. Also note that the boundedness implies that there are finitely many distinct walls, as the coefficients of the equations are determined by the topological type of $\mathcal{F}$.

Lemma 3.6.2. Let $\mathcal{E}$ denote a purely $d$-dimensional and $(p, \underline{L})$-regular quiver sheaf of topological type $\tau$ which satisfies the relations $I$, and consider a quiver subsheaf $\mathcal{F} \subset \mathcal{E}$ contained in the family $S$. Suppose that $\sigma$ and $\sigma^{\prime}$ are stability conditions in $\Sigma^{\prime}$ which are contained in the same chamber $C$. Then

$$
p_{\mathcal{F}}^{\sigma} \leq p_{\mathcal{E}}^{\sigma} \text { if and only if } p_{\mathcal{F}}^{\sigma^{\prime}} \leq p_{\mathcal{E}}^{\sigma^{\prime}} .
$$

A similar assertion holds for strict inequality.

Proof. This is a variant of the proof of Lemma 4.6 in [GRT16]. For brevity, we concentrate on the case of non-strict inequality.

We may write

$$
p_{\mathcal{F}}^{\sigma}-p_{\mathcal{E}}^{\sigma}=\sum_{s=0}^{d} \frac{c_{s}^{\sigma}}{s !} T^{s} \in \mathbb{R}[T] .
$$

Assuming that $p_{\mathcal{F}}^{\sigma} \leq p_{\mathcal{E}}^{\sigma}$ but $p_{\mathcal{F}}^{\sigma^{\prime}}>p_{\mathcal{E}}^{\sigma^{\prime}}$, we can choose a minimal index $e$ such that $c_{t}^{\sigma}=c_{t}^{\sigma^{\prime}}=0$ for all $t>e$, so that

$$
c_{e}^{\sigma} \leq 0 \text { and } c_{e}^{\sigma^{\prime}} \geq 0,
$$


but not both vanish at the same time. The function

$$
f(\sigma)=\frac{\sum_{i \in Q_{0}} \sum_{j=1}^{N} \sigma_{i j} \alpha_{e}^{L_{j}}\left(\mathcal{F}_{i}\right)}{\sum_{k \in Q_{0}} \sum_{l=1}^{N} \sigma_{k l} \alpha_{d}^{L_{l}}\left(\mathcal{F}_{k}\right)}-\frac{\sum_{i \in Q_{0}} \sum_{j=1}^{N} \sigma_{i j} \alpha_{e}^{L_{j}}\left(\mathcal{E}_{i}\right)}{\sum_{k \in Q_{0}} \sum_{l=1}^{N} \sigma_{k l} \alpha_{d}^{L_{l}}\left(\mathcal{E}_{k}\right)}
$$

is continuous in $\sigma$, has the wall $W_{e, \mathcal{F}}$ as vanishing locus, and satisfies $c_{e}^{\sigma}=$ $f(\sigma)$. Hence, either $\sigma$ or $\sigma^{\prime}$ is not an element of $W_{e, \mathcal{F}}$, so that $C$ is not contained in the wall. But if neither $c_{e}^{\sigma}$ nor $c_{e}^{\sigma^{\prime}}$ vanishes, they have differing signs so that $f\left(\sigma^{\prime \prime}\right)=0$ for some $\sigma^{\prime \prime}$ on a connecting path from $\sigma$ to $\sigma^{\prime}$ inside $C$. In any case, we deduce that $C \cap W_{e, \mathcal{F}} \neq \emptyset$, which is a contradiction.

Proposition 3.6.3. Consider $\Sigma, \Sigma^{\prime}, p, d, \tau$ and $\underline{L}$ as explained above. Then, the finite chamber structure $W=\left(W_{e, \mathcal{F}}\right)$ encodes the equivalence of stability conditions. More precisely, for any stability conditions $\sigma$ and $\sigma^{\prime}$ inside $\Sigma^{\prime}$ which belong to the same chamber $C$, and for any purely d-dimensional and $(p, \underline{L})$-regular quiver sheaves $\mathcal{E}$ and $\mathcal{E}^{\prime}$ of topological type $\tau$ which satisfy the relations $I$, the following assertions hold.

(1) $\mathcal{E}$ is $\sigma$-semistable if and only if $\mathcal{E}$ is $\sigma^{\prime}$-semistable, and the same assertion holds for stability.

(2) Let $\mathcal{E}$ and $\mathcal{E}^{\prime}$ be semistable with respect to both $\sigma$ and $\sigma^{\prime}$. Then $\mathcal{E}$ is $S$-equivalent to $\mathcal{E}^{\prime}$ with respect to $\sigma$ if and only if it is with respect to $\sigma^{\prime}$.

Proof. The proof is the same as the corresponding proof of Proposition 4.2 in [GRT16], where we use our Lemma 3.6.2.

COROLlary 3.6.4. The chamber structure on $\Sigma^{\prime}$ as in Proposition 3.6.3 induces a finite partition of $\Sigma$ such that the behavior of stability and $S$ equivalence remains unchanged as $\sigma$ varies within any fixed block of the partition.

Proposition 4.2 in [GRT16] is a special case of our Proposition 3.6.3 in the case of the trivial quiver $Q=\bullet$ and torsion-free sheaves on an integral scheme. In contrast to their result, we can not guarantee the existence of rational points in the chambers, though in the special case of symmetric stability conditions, discussed in Section 3.7 we can. For our variation results however, we need to assume the existence of rational points.

Assumption 3.6.5. Each chamber on $\Sigma^{\prime}$ contains a rational point. In that case, we can replace any stability condition $\sigma$ by a rational one without loss of generality.

REMARK 3.6.6. The relations we used in our definition of $S$ select a subfamily from the corresponding family $S^{\prime}$ wich we get without imposing the relations. The chamber structure without relations is thus a refinement of the chamber structure with relations.

As in [GRT16], we think of $\Sigma^{\prime}$ as a bounded set of stability parameters, so that the integer $p$ could then be chosen so that all semistable sheaves with respect to some $\sigma \in \Sigma^{\prime}$ are $(p, \underline{L})$-regular.

The cone $\Sigma^{\prime}$ is just an auxiliary object. Its purpose is to ensure the boundedness of the family $S$ (compare with the proof of the Grothendieck Lemma 3.1 .21 ), so that there are only finitely many walls. A priori it seems possible 
that for the whole set $\left(\mathbb{R}^{Q_{0} \times N}\right)$, this is no longer true, i.e. that the walls accumulate at the boundary.

It is also true that the partition of $\Sigma$ which is induced by the chamber structure on $\Sigma^{\prime}$ does not essentially depend on the choice of $\Sigma^{\prime}$. We say that two conditions $\sigma$ and $\sigma^{\prime}$ in $\Sigma$ are equivalent if for any purely $d$-dimensional quiver sheaves $\mathcal{E}$ and $\mathcal{E}^{\prime}$ of topological type $\tau$ we have

(1) $\mathcal{E}$ is $\sigma$-semistable if and only if $\mathcal{E}$ is $\sigma^{\prime}$-semistable,

(2) $\mathcal{E}$ and $\mathcal{E}^{\prime}$ are $S$-equivalent with respect to $\sigma$ if and only if they are $S$-equivalent with respect to $\sigma^{\prime}$.

COROLlary 3.6.7. The partition of $\Sigma$ induced by a chamber structure on $\Sigma^{\prime}$, as in Corollary 3.6.4, is a refinement of the partition of $\Sigma$ into equivalence classes. In particular, the latter is finite.

REMARK 3.6.8. Even though we can not ensure the existence of rational points in the chambers, we can ensure the existence of points with real algebraic entries. Such points are even dense within each chamber.

To see this, consider a real closed field $K \subset \mathbb{R}$, eg. $K=\mathbb{R}$ or $K$ equal to the set of real algebraic numbers. We note that each chamber in $\Sigma^{\prime}$ is an open subset of a real algebraic variety $Z$, which is defined by polynomials (of degree two) with rational coefficients. Hence, for any real closed field $K \subset \mathbb{R}$, any fixed rational point $x \in \mathbb{R}^{Q_{0} \times N}$, and any rational number $\varepsilon>0$, the statement

$$
\left(S_{x, \varepsilon}, K\right): Z(K) \cap B_{\varepsilon}(x) \text { is non-empty }
$$

is a statement of elementary arithmetic in the sense of Tarski (see [Ta98]), with variables which take values in $K$, and not containing free variables. According to [Ta98], the theory of elementary arithmetic is quantifiereliminable, so that $\left(S_{x, \varepsilon}, K\right)$ is equivalent to a statement $\left(S_{x, \varepsilon}^{\prime}\right)$ which does not contain any variables at all. In particular, $\left(S_{x, \varepsilon}^{\prime}\right)$ is independent of the choice of a model $K$ for the axioms of a real closed field. Consequently, the same is true for $\left(S_{x, \varepsilon}, K\right)$. Now, $Z(K) \subset Z=Z(\mathbb{R})$ is dense if

$$
\forall x \in \mathbb{Q}^{Q_{0} \times N_{\forall}} \forall \varepsilon \in \mathbb{Q}_{>0}: Z(K) \cap B_{\varepsilon}(x) \neq \emptyset \Leftrightarrow Z \cap B_{\varepsilon}(x) \neq \emptyset,
$$

which, as we have seen above, is a true statement.

\subsubsection{Variation of moduli spaces.}

We now want to describe the behavior of the moduli spaces when changing the stability condition. For this to work, we need the moduli spaces $M_{\tau}^{\sigma-s s t}(X, Q, I)$ to be projective. Hence, we assume that $Q$ contains no oriented cycles, which also implies that $Q\left(Q^{\prime}\right)$ does not contain oriented cycles (Lemma 3.2.3). Furthermore, we assume that $X$ is smooth, and that Assumption 3.6.5 holds, ie. that each chamber contains a rational point. 
Let $\Sigma \subset \mathbb{R}_{>0}^{Q_{0} \times N}$ be a finite subset of rational, positive, and bounded stability conditions under a given set of relations $I$, and choose $m \gg n \gg p \gg 0$ so that the conditions (1)-(4) in the beginning of Section 3.4 are satisfied for all $\sigma \in \Sigma$. Let

$$
R_{I^{\prime}}=R_{d}\left(Q\left(Q^{\prime}\right), H, I^{\prime}(I)\right)
$$

be the representation variety of $Q\left(Q^{\prime}\right)$ with labeling $H$ and relations $I^{\prime}(I)$, where $d$ is the dimension vector determined by $\tau, m$ and $n$. This embeds as a closed subvariety into the representation variety $R=R_{d}\left(Q\left(Q^{\prime}\right), H\right)$. Moreover, for $\sigma \in \Sigma$ let $\theta=\theta(d, \sigma)$ be the corresponding parameter defined in Section 3.3, and let

$$
B^{[\sigma-s s t]} \subseteq R_{I^{\prime}}
$$

denote the locally closed subset of $R_{I^{\prime}}$ that parametrizes $\theta$-semistable points in $R_{I^{\prime}}$ that lie in the image of the embedding functor $\operatorname{Hom}(T, *)$ from Section 3.2 .2 , i.e., $\theta$-semistable points that come from $\sigma$-semistable quiver sheaves of topological type $\tau$ which satisfy the relations $I$. Then, $B^{[\sigma-s s t]}$ is also a locally closed, and $G$-invariant, subset of $R$. Define

$$
Z=\overline{\bigcup_{\sigma \in \Sigma} B^{[\sigma-s s t]}} \text {. }
$$

TheOrem 3.6.9. Let $Z$ be as above. Then,

$$
Z^{\theta-s s t}=R^{\theta-s s t} \cap Z=B^{[\sigma-s s t]},
$$

and the moduli space $M_{\tau}^{\sigma-s s t}(X, Q, I)$ is given by the GIT quotient

$$
M_{\tau}^{\sigma-s s t}(X, Q, I)=Z^{\theta-s s t} / / G .
$$

Proof. First of all, by the same argument as in the proof of Theorem 10.1 of [GRT16], it follows that $B^{[\sigma-s s t]}$ is a dense open subset of $Z^{\theta-s s t}$.

Now, by the assumption that $Q$ has no oriented cycles, the same holds for the twisted quiver $Q\left(Q^{\prime}\right)$, and hence the quotient $R^{\sigma-s s t} / / G$ is projective. Since $Z$ is a closed and $G$-invariant subscheme, the GIT quotient $Z^{\theta-s s t} / / G$ embeds as a closed subscheme of $R^{\theta-s s t} / / G$ and is thus also projective. Since $M_{\tau}^{\sigma-s s t}(X, Q, I)=B^{[\sigma-s s t]} / / G$ and $Z^{\theta-s s t} / / G$ are both projective, the image of $M_{\tau}^{\sigma-s s t}(X, Q, I)=B^{[\sigma-s s t]} / / G$ in $Z^{\theta-s s t} / / G$ by the morphism induced by the inclusion $B^{[\sigma-s s t]} \subseteq Z^{\theta-s s t}$ is closed. Being also dense, this image has to coincide with $Z^{\theta-s s t} / / G$.

Let $\pi: Z^{\theta-\text { sst }} \rightarrow Z^{\theta-s s} / / G$ denote the quotient morphism. By the comparison of $S$-equivalence (Theorem 3.3.23), for each $G$-orbit $\mathcal{O}$ in $B^{[\sigma-s s t]}$, the closed orbit $\mathcal{O}^{\prime}$ in the orbit closure $\overline{\mathcal{O}}$ lies in $B^{[\sigma-s s t]}$. Hence, we can apply [ACK07], Lemma 6.2, to conclude that $\pi$ restricts to a good quotient $B^{[\sigma-s s t]} \rightarrow B^{[\sigma-s s t]} / / G$, and that $\pi^{-1}\left(\pi\left(B^{[\sigma-s s t]}\right)\right)=B^{[\sigma-s s t]}$. Together with the above established identity $\pi\left(B^{[\sigma-s s t]}\right)=Z^{\theta-s s t}$, this implies that $B^{[\sigma-s s t]}=Z^{\theta-s s t}$.

We obtain a Mumford-Thaddeus principle for $\sigma$-semistable quiver sheaves, with essentially the same proof as in [GRT16]. Note that the Thaddeus flips occurring here are to be interpreted in the context of VGIT of $Z$. 
COROLlaRY 3.6.10. Let $\sigma$ and $\sigma^{\prime}$ be bounded stability parameters under some given set of relations $I$. Then the moduli spaces

$$
M_{\tau}^{\sigma-s s t}(X, Q, I) \text { and } M_{\tau}^{\sigma-s s t}(X, Q, I)
$$

are related by a finite sequence of Thaddeus flips induced by VGIT of the variety $Z$.

\subsection{Symmetric stability conditions}

The results about construction and variation of the moduli spaces, as outlined in Sections 3.4 and 3.6, demand that the family of semistable sheaves under consideration is bounded. In general, we can not guarantee that this is true, though in the case that $\sigma_{i j}$ does not depend on the vertex $i \in Q_{0}$, we can build upon the boundedness results of [GRT16].

Recall that

$$
\sigma_{i}=\left(\sigma_{i j}\right)_{j \in\{1, \ldots, N\}}
$$

is the restriction of $\sigma$ to the vertex $i \in Q_{0}$. For the remainder of this section we make the following assumption, which could also be understood as $\sigma$ being symmetric under permutations of $Q_{0}$.

Assumption 3.7.1. The tuples $\sigma_{i} \in \mathbb{R}_{\geq 0}^{N} \backslash 0$, for $i \in Q_{0}$, all coincide.

Under this assumption on $\sigma$, we let $\hat{\sigma} \in \mathbb{R}^{N}$ denote this common restriction of $\sigma$. As a technical tool, we consider $Q_{0}$-tuples

$$
\left(\mathcal{E}_{i}\right)_{i \in Q_{0}}
$$

of coherent sheaves on $X$, following an idea of Schmitt (cf. [Sch05]). Tupels can be identified with quiver sheaves for the quiver with the set of vertices $Q_{0}$ and with no arrows. In particular, if $\mathcal{E}$ is a quiver sheaf, we obtain a $Q_{0^{-}}$ tuple $\left(\mathcal{E}_{i}\right)_{i \in Q_{0}}$ of coherent sheaves by forgetting the morphisms $\mathcal{E}_{\alpha}: \mathcal{E}_{i} \rightarrow \mathcal{E}_{j}$ given by the arrows $\alpha: i \rightarrow j$ of $Q$. By a subtuple of a $Q_{0}$-tuple $\left(\mathcal{E}_{i}\right)_{i \in Q_{0}}$ we mean a $Q_{0}$-tuple $\left(\mathcal{F}_{i}\right)_{i \in Q_{0}}$, where $\mathcal{F}_{i}$ is a coherent subsheaf of $\mathcal{E}_{i}$ for each $i$. If $\sigma \in\left(\mathbb{R}^{Q_{0} \times N}\right)_{+}$, we say that a $Q_{0}$-tuple $\left(\mathcal{E}_{i}\right)_{i \in Q_{0}}$ is $\sigma$-semistable if it is pure of some dimension and the inequality

$$
p_{\mathcal{F}}^{\sigma} \leq p_{\mathcal{E}}^{\sigma}
$$

holds for all nontrivial subtuples $\left(\mathcal{F}_{i}\right)_{i \in Q_{0}}$ of $\left(\mathcal{E}_{i}\right)_{i \in Q_{0}}$.

Let $E$ denote any coherent sheaf on $X$, and pick any vertex $i_{0} \in Q_{0}$. Then we define the quiver sheaf $\delta_{i_{0}}(E)$ by

$$
\delta_{i_{0}}(E)_{i}=\left\{\begin{array}{ll}
E & i=i_{0} \\
0 & i \neq i_{0}
\end{array}, \delta_{i_{0}}(E)_{\alpha}=0 .\right.
$$

It is very easy to verify that

$$
p_{\delta_{i_{0}}(E)}^{\sigma}=p_{E}^{\sigma_{i_{0}}} .
$$

Lemma 3.7.2. Let $\mathcal{E}$ denote a $\sigma$-semistable tuple of sheaves on $X$. Then

$$
p_{\mathcal{E}}^{\sigma}=p_{\mathcal{E}_{i}}^{\sigma_{i}}
$$

for all vertices $i \in Q_{0}$. Moreover, all $\mathcal{E}_{i}$ are $\sigma_{i}$-semistable. 
Proof. Clearly, $\delta_{i_{0}}\left(\mathcal{E}_{i_{0}}\right)$ is both a subobject and a quotient of $\mathcal{E}$ in the category of tuples. Thus the equality of the Hilbert polynomials follows.

If $U \subset \mathcal{E}_{i}$ is any subsheaf, then $\delta_{i}(U)$ is also a subobject of the tuple $\mathcal{E}$. Hence

$$
p_{U}^{\sigma_{i}}=p_{\delta_{i}(U)}^{\sigma} \leq p_{\mathcal{E}}^{\sigma}=p_{\mathcal{E}_{i}}^{\sigma_{i}}
$$

REMARK 3.7.3. The condition

$$
p_{\mathcal{E}}^{\sigma}=p_{\mathcal{E}_{i}}^{\sigma_{i}}
$$

of course implies the equality of degrees of these polynomials. Since we require that $\sigma \in\left(\mathbb{R}^{Q_{0} \times N}\right)_{+}$, this implies that the dimensions

$$
\operatorname{dim}\left(\mathcal{E}_{i}\right), i \in Q_{0},
$$

all coincide.

LEMMA 3.7.4. Let $\left(\mathcal{E}_{i}\right)_{i \in Q_{0}}$ and $\left(\mathcal{F}_{i}\right)_{i \in Q_{0}}$ be $\sigma$-semistable tuples and assume that $p_{\mathcal{E}}^{\sigma}>p_{\mathcal{F}}^{\sigma}$. Then

$$
\operatorname{Hom}\left(\mathcal{E}_{i}, \mathcal{F}_{k}\right)=0
$$

for all $i, k \in Q_{0}$.

Proof. Let $i, k \in Q_{0}$. By Lemma 3.7.2, $\mathcal{E}_{i}$ and $\mathcal{F}_{k}$ are both semistable with respect to $\hat{\sigma}$, and $p_{\mathcal{E}_{i}}^{\hat{\sigma}}>p_{\mathcal{F}_{k}}^{\hat{\sigma}}$. The claim then follows from general properties of stability conditions (cf. [HL10], Proposition 1.2.7 and its proof).

Although we are interested in quiver sheaves and tuples, we formulate our next lemma in the general setting of stability conditions on abelian categories (cf. [R97]).

LEMMA 3.7.5. Let

$$
0=\mathcal{E}_{0} \subset \mathcal{E}_{1} \subset \cdots \subset \mathcal{E}_{r}=\mathcal{E}
$$

denote the Harder-Narasimhan filtration for some object $\mathcal{E}$ with respect to some semistability condition $\mu$ (in the sense of [R97]).

Then, for all $0<i<r$ we have

$$
\mu\left(\mathcal{E}_{i}\right)>\mu(\mathcal{E}) .
$$

Proof. We proceed by induction on $i$.

If the filtration is nontrivial, we clearly have $\mu\left(\mathcal{E}_{1}\right)>\mu(\mathcal{E})$. Now suppose that $\mu\left(\mathcal{E}_{i}\right)>\mu(\mathcal{E})$ is already settled, that is, $\mu(\mathcal{E})>\mu\left(\mathcal{E} / \mathcal{E}_{i}\right)$. The HarderNarasimhan filtration of $\mathcal{E} / \mathcal{E}_{i}$ is given by

$$
0 \subset \mathcal{E}_{i+1} / \mathcal{E}_{i} \subset \cdots \subset \mathcal{E} / \mathcal{E}_{i}
$$

and by definition $\mathcal{E}_{i+1} / \mathcal{E}_{i}$ is then a maximal destabilizing subobject of $\mathcal{E} / \mathcal{E}_{i}$, so that

$$
\mu\left(\mathcal{E}_{i+1} / \mathcal{E}_{i}\right)>\mu\left(\mathcal{E} / \mathcal{E}_{i}\right) .
$$

The short exact sequence

$$
0 \rightarrow \mathcal{E}_{i+1} / \mathcal{E}_{i} \rightarrow \mathcal{E} / \mathcal{E}_{i} \rightarrow \mathcal{E} / \mathcal{E}_{i+1} \rightarrow 0
$$

then yields

$$
\mu\left(\mathcal{E} / \mathcal{E}_{i}\right)>\min \left\{\mu\left(\mathcal{E}_{i+1} / \mathcal{E}_{i}\right), \mu\left(\mathcal{E} / \mathcal{E}_{i+1}\right)\right\}=\mu\left(\mathcal{E} / \mathcal{E}_{i+1}\right),
$$


where the equality holds by virtue of (1). Hence,

$$
\mu(\mathcal{E})>\mu\left(\mathcal{E} / \mathcal{E}_{i}\right)>\mu\left(\mathcal{E} / \mathcal{E}_{i+1}\right),
$$

which then implies that $\mu\left(\mathcal{E}_{i+1}\right)>\mu(\mathcal{E})$. The claim thus follows by induction.

Proposition 3.7.6. Assume that $\sigma$ satisfies Assumption 3.7.1. Let $\mathcal{E}$ denote any pure quiver sheaf. Then $\mathcal{E}$ is $\sigma$-semistable as a quiver sheaf if and only if $\mathcal{E}$ is $\sigma$-semistable as a $Q_{0}$-tuple of sheaves on $X$.

In that case, all sheaves $\mathcal{E}_{i}$ are $\hat{\sigma}$-semistable. Moreover, the numbers $\operatorname{dim}\left(\mathcal{E}_{i}\right)$ all coincide.

Proof. We first assume that $\mathcal{E}$ is semistable as a quiver sheaf and show that it is then semistable as a tuple. Let therefore

$$
0=\mathcal{F}^{0} \subset \mathcal{F}^{1} \subset \cdots \subset \mathcal{F}^{r}=\mathcal{E}
$$

denote the Harder-Narasimhan filtration in the category of tuples. We assume that this filtration is not trivial, i.e., $r \geq 2$.

Because $p_{\mathcal{F} k}^{\sigma}>p_{\mathcal{E}}^{\sigma}$ for all $k$, by Lemma 3.7.5, none of the $\mathcal{F}^{k}$ can be a quiver subsheaf of $\mathcal{E}$, so that for each index $k$ there exists at least one arrow $(\alpha: i \rightarrow j)=\left(\alpha^{k}: i^{k} \rightarrow j^{k}\right)$ such that

$$
\mathcal{E}_{\alpha}\left(\mathcal{F}_{i}^{k}\right) \nsubseteq \mathcal{F}_{j}^{k} .
$$

Fix one such $k$ and $\alpha: i \rightarrow j$ and choose $l$ minimal such that $\mathcal{E}_{\alpha}\left(\mathcal{F}_{i}^{k}\right) \subseteq \mathcal{F}_{j}^{l}$. By choice of $\alpha$ we clearly have $l \geq k+1$. Furthermore, choose $m$ maximal such that $\mathcal{F}_{i}^{m} \subseteq \operatorname{ker}\left(\mathcal{E}_{\alpha}\right)$. Again, the choice of $\alpha$ implies $m \leq k-1$. Then we have a well-defined induced morphism

$$
f: \mathcal{F}_{i}^{m+1} / \mathcal{F}_{i}^{m} \rightarrow \mathcal{F}_{j}^{l} / \mathcal{F}_{j}^{l-1}
$$

which does not vanish by the choice of $m$ and $l$. Note that

$$
\mathcal{F}_{i}^{m-1} / \mathcal{F}_{i}^{m}=\left(\mathcal{F}^{m-1} / \mathcal{F}^{m}\right)_{i}
$$

is $\hat{\sigma}$-semistable according to Lemma 3.7.2, with the same multi-Hilbert polynomial as the corresponding subquotient of tuples. Now,

$$
\begin{aligned}
p_{\mathcal{F}^{k} / \mathcal{F}^{k-1}}^{\sigma} & \leq p_{\mathcal{F}^{m+1} / \mathcal{F}^{m}}^{\sigma} \\
& \leq p_{\mathcal{F}^{l} / \mathcal{F}^{l-1}}^{\sigma} \\
& \leq p_{\mathcal{F}^{k+1} / \mathcal{F}^{k}}^{\sigma},
\end{aligned}
$$

where the first and third inequalities follow by the properties of the HarderNarasimhan filtration, and the second inequality follows from Lemma 3.7.4 because of the existence of the nonzero morphism $f$. Now, the inequality

$$
p_{\mathcal{F} k / \mathcal{F} k-1}^{\sigma} \leq p_{\mathcal{F} k+1 / \mathcal{F} k}^{\sigma}
$$

is a contradiction. Hence, $\mathcal{E}$ is already semistable as a tuple.

The other implication is trivial, and the question of semistability of the $\mathcal{E}_{i}$ and their dimensions is taken care of by Lemma 3.7.2 and Remark 3.7.3. 
Under the assumption of this section we can now show a boundedness result for families of semistable quiver sheaves for certain varieties $X$, building on the work of [GRT16]. Note that this also implies the boundedness of the subfamily defined by any set of relations $I$.

THEOREM 3.7.7. Let $X$ be a smooth projective variety, and let $\tau \in B(X)_{\mathbb{Q}}^{Q_{0}}$ be a fixed topological type. Assume further that one of the following conditions holds:

(1) $\operatorname{dim}(X) \leq 3$,

(2) the Picard rank of $X$ is at most two,

(3) all the sheaves under consideration are of rank at most two.

Then, for any subset $\Sigma \subseteq\left(\mathbb{R}^{Q_{0} \times N}\right)_{+}$of parameters $\sigma$ satisfying Assumption 3.7.1, the family of all quiver sheaves of topological type $\tau$ which are semistable with respect to some $\sigma \in \Sigma$ is bounded.

Proof. By Proposition 3.7.6, the claim follows from the corresponding boundedness result for $\hat{\sigma}$-semistable sheaves in [GRT16], Corollary 6.12.

Clearly, the set of stability conditions $\sigma$ which satisfy Assumption 3.7.1 form a subset $\Sigma_{0}$ of $\left(\mathbb{R}^{Q_{0} \times N}\right)$, which is convex. Hence, it is possible to move from one such condition to another without leaving this subset. At least for torsion-free quiver sheaves, the walls encoding the change of stability are hyperplanes.

Proposition 3.7.8. Let $\tau$ denote a topological type of torsion-free quiver sheaves on an integral and projective scheme $X$, and let $p$ be a natural number. Then, for any closed polyhedral cone $\Sigma^{\prime} \subset \Sigma_{0}$, the walls, as given by Subsection 3.6.1, are rational hyperplanes. In particular, each chamber in $\Sigma^{\prime}$ contains a rational point.

Proof. Consider the wall $W_{e, \mathcal{F}}$ associated to the quiver subsheaf $\mathcal{F} \subset \mathcal{E}$ and integer $e$, which is defined by the equation

$$
\frac{\sum_{i \in Q_{0}} \sum_{j=1}^{N} \sigma_{i j} \alpha_{e}^{L_{j}}\left(\mathcal{F}_{i}\right)}{\sum_{k \in Q_{0}} \sum_{l=1}^{N} \sigma_{k l} \alpha_{d}^{L_{l}}\left(\mathcal{F}_{k}\right)}-\frac{\sum_{i \in Q_{0}} \sum_{j=1}^{N} \sigma_{i j} \alpha_{e}^{L_{j}}\left(\mathcal{E}_{i}\right)}{\sum_{k \in Q_{0}} \sum_{l=1}^{N} \sigma_{k l} \alpha_{d}^{L_{l}}\left(\mathcal{E}_{k}\right)}=0 .
$$

Because $X$ is integral, and each sheaf $\mathcal{E}_{k}$ is torsion-free, we can write $\alpha_{d}^{L_{l}}\left(\mathcal{F}_{k}\right)=\alpha_{d}^{L_{l}}\left(\mathcal{O}_{X}\right) \operatorname{rk}\left(\mathcal{F}_{k}\right)$, and similarly for the $\mathcal{E}_{k}$ (compare with [HL10], Definition 1.2.2). Note that the rank does not depend on the choice of an ample line bundle. Further, by Assumption 3.7.1, we can write $\sigma_{i j}=\hat{\sigma}_{j}$, so that we may rewrite the equation as

$$
f(\sigma) \frac{\sum_{i \in Q_{0}} \sum_{j=1}^{N} \sigma_{i j}\left(\operatorname{rk}(\mathcal{E}) \alpha_{e}^{L_{j}}\left(\mathcal{F}_{i}\right)-\operatorname{rk}(\mathcal{F}) \alpha_{e}^{L_{j}}\left(\mathcal{E}_{i}\right)\right)}{\operatorname{rk}(\mathcal{F}) \operatorname{rk}(\mathcal{E})}=0
$$


Here, we introduce the notation

$$
\begin{aligned}
\operatorname{rk}(\mathcal{E}) & =\sum_{i \in Q_{0}} \operatorname{rk}\left(\mathcal{E}_{i}\right), \\
f(\sigma) & =\left(\sum_{k \in Q_{0}} \sum_{l=1}^{N} \hat{\sigma}_{l} \alpha_{d}^{L_{l}}\left(\mathcal{O}_{X}\right)\right)^{-1} .
\end{aligned}
$$

The function $f$ is always positive, and hence can be ignored for the definition of the wall. The remaining equation is linear in the entries $\sigma_{i j}$.

COROLlary 3.7.9. Let $X$ denote a smooth projective variety, and let $\tau$ denote a fixed topological type for torsion-free quiver sheaves. Assume that one of the conditions of Theorem 3.7 .7 holds. Then, for any stability conditions $\sigma, \sigma^{\prime} \in \Sigma_{0}$ the moduli spaces

$$
M_{\tau}^{\sigma-\mathrm{sst}}(X, Q) \text { and } M_{\tau}^{\sigma^{\prime}-\mathrm{sst}}(X, Q)
$$

exist. Moreover, if $Q$ does not contain oriented cycles, these moduli spaces are projective, and are related by a finite sequence of Thaddeus flips, induced by VGIT of the variety $Z$ as in Theorem 3.6.9.

\subsection{Quiver Quot-Schemes}

We construct the quiver sheaf version of the Quot-scheme, which is helpful as a technical tool throughout this chapter. This section is mostly independent of the other sections.

First, we need a version of the Quot-scheme for sheaves which uses the topological type instead of the Hilbert polynomials.

Recall the strategy of proof in the construction of the sheaf version of the Quot-scheme Quot ${ }_{E / X / S}^{P}$, parametrizing flat quotients of the sheaf $E$ with Hilbert polynomial $P$ (eg. consider [HL10], Theorem 2.2.4). Reducing to the case

$$
X=\mathbb{P}^{n} \rightarrow S=\operatorname{Spec}(k),
$$

one constructs an embedding of functors

$$
\mathcal{Q u o t}_{E / X / S}^{P} \rightarrow \mathcal{G r a s s}_{k}\left(H^{0}(E(m)), P\right),
$$

where the right hand side is represented by the Grassmannian scheme. The Quot-scheme is then given as the component corresponding to $P$ of the flattening stratification of some suitable sheaf $F$ on the Grassmannian.

This proof carries over to the case of the Quot-scheme Quot $_{E / X / S}^{\tau}$, parameterizing flat quotients of $E$ with topological type $\tau \in B(X)_{\mathbb{Q}}$. More precisely, the same construction as above gives an embedding

$$
\mathcal{Q u o t}_{E / X / S}^{\tau} \rightarrow \mathcal{G r a s s}_{k}\left(H^{0}(E(m)), P\right),
$$

where $P=P(\tau)$ is the Hilbert polynomial determined by $\tau$ (and the implicitly fixed relatively very ample line bundle). Since $\tau$ is locally constant in flat families, we may consider the component of the flattening stratification 
corresponding to $\tau$. The same argument as above shows that this component represents the Quot-functor.

We will mainly need the generalization to the Quiver Quot-scheme in the version using the topological type. Though all arguments hold for the version using Hilbert polynomials equally well.

Definition 3.8.1. Consider a projective scheme $X \rightarrow S$ over a noetherian base scheme $S$, a topological type $\tau \in B(X)_{\mathbb{Q}}^{Q_{0}}$ and a quiver sheaf $\mathcal{E}$ on $X$. The Quiver Quot-functor

$$
\mathcal{Q u o t}_{\mathcal{E} / X / S}^{\tau}:(S c h / S)^{\text {op }} \rightarrow \text { Sets }
$$

assigns to a scheme $T \rightarrow S$ the set of equivalence classes of quotient quiver sheaves $q: \mathcal{E}_{T} \rightarrow \mathcal{F}$ on $X_{T}$ where $\mathcal{F}$ is flat over $T$ and the topological type of the fiberwise quiver sheaves $(\mathcal{E})_{t}$ are equal to $\tau$.

Definition 3.8.2. Consider a projective scheme $X \rightarrow S$ over a noetherian base scheme $S$, a tuple of polynomials $P \in \mathbb{Q}[T]^{Q_{0}}$ and a quiver sheaf $\mathcal{E}$ on $X$. Also, implicitly fix a relatively very ample line bundle on $X$. The Quiver Quot-functor

$$
\operatorname{Quot}_{\mathcal{E} / X / S}^{P}:(S c h / S)^{\mathrm{op}} \rightarrow \text { Sets }
$$

assigns to a scheme $T \rightarrow S$ the set of equivalence classes of quotient quiver sheaves $q: \mathcal{E}_{T} \rightarrow \mathcal{F}$ on $X_{T}$ where $\mathcal{F}$ is flat over $T$ and the Hilbert polynomials of the fibrewise sheaves at the vertices $\left(\mathcal{E}_{i}\right)_{t}$ are equal to $P_{i}$.

Proposition 3.8.3. Let $X \rightarrow S$ denote a projective scheme over a noetherian base scheme $S$, and let $E$ and $F$ denote coherent sheaves on $X$ such that $F$ is flat over $S$. Consider a morphism $f: E \rightarrow F$ and the functor

$$
F:(S c h / S)^{\mathrm{op}} \rightarrow \text { Sets, }(T \rightarrow S) \mapsto\left\{\phi \in \operatorname{Hom}_{S}(T, X) \mid \Phi^{*}(f)=0\right\} .
$$

This functor is represented by a closed subscheme of $X$.

Proof. Consider the functor

$$
F^{\prime}:(S c h / S)^{\mathrm{op}} \rightarrow \text { Sets, }(T \rightarrow S) \mapsto \operatorname{Hom}_{X_{T}}\left(E_{T}, F_{T}\right)
$$

as in [N05], Theorem 5.8. It is represented by a scheme $V=\operatorname{Spec}\left(\operatorname{Sym}_{\mathcal{O}_{S}}(Q)\right)$ for a suitable sheaf $Q$ on $S$.

In our situation, $f \in F^{\prime}(X)$ corresponds to a morphism $\phi_{f}: X \rightarrow V$, so that Remark 5.9 in [N05] implies that our functor $F$ is represented by the closed subscheme $\phi_{f}^{-1}\left(V_{0}\right)$, where $V_{0} \subset V$ is the image of the zero section $S \rightarrow V$.

THEOREM 3.8.4. The Quiver Quot-functor is represented by a projective scheme $\operatorname{Quot}_{\mathcal{E} / X / S}^{\tau}$, the so called Quiver Quot-scheme. It is given as the closed subscheme

$$
\operatorname{Quot}_{\mathcal{E} / X / S}^{\tau} \subset \prod_{i \in Q_{0}} \operatorname{Quot}_{\mathcal{E}_{i} / X / S}^{\tau_{i}}
$$

which satisfies the conditions to be compatible with the morphisms $\mathcal{E}_{\alpha}$. A similar statement holds for the version using Hilbert polynomials. 
Proof. By projection to the quotient sheaves at the vertices we get a natural transformation of functors

$$
\mathcal{Q} \operatorname{uot}_{\mathcal{E} / X / S}^{\tau} \rightarrow \prod_{i \in Q_{0}} \mathcal{Q u o t}_{\mathcal{E}_{i} / X / S}^{\tau_{i}},
$$

where the right hand side is clearly represented by the projective scheme

$$
G=\prod_{i \in Q_{0}} G_{i}=\prod_{i \in Q_{0}} \operatorname{Quot}_{\mathcal{E}_{i} / X / S}^{\tau_{i}} .
$$

Consider the universal quotients at the vertices

$$
q_{i}:\left(\mathcal{E}_{i}\right)_{G_{i}} \rightarrow \mathcal{U}_{i}
$$

on the Quot-schemes $G_{i}$, and denote by $e_{i}: \mathcal{K}_{i} \rightarrow\left(\mathcal{E}_{i}\right)_{G_{i}}$ their kernels. For a morphism

$$
\phi: T \rightarrow G
$$

of noetherian schemes over $S$ and any arrow $\alpha: i \rightarrow j$ we obtain the following exact diagram via pullback.

$$
\begin{aligned}
& \Phi^{*} \mathcal{K}_{i} \stackrel{\Phi^{*} e_{i}}{\longrightarrow}\left(\mathcal{E}_{i}\right)_{T} \stackrel{\Phi^{*} q_{i}}{\longrightarrow} \Phi^{*} \mathcal{U}_{i} \longrightarrow 0 \\
& \Phi^{*} \mathcal{K}_{j} \stackrel{\Phi^{*} e_{j}}{\longrightarrow}\left(\mathcal{E}_{j}\right)_{T} \stackrel{\Phi^{*} q_{j}}{\longrightarrow} \Phi^{*} \mathcal{U}_{j} \longrightarrow 0
\end{aligned}
$$

Hence the morphism $\left(\mathcal{E}_{\alpha}\right)_{T}$ descends to the quotients if and only if

$$
0=\Phi^{*} q_{j}\left(\mathcal{E}_{\alpha}\right)_{T} \Phi^{*} e_{i}=\Phi^{*}\left(q_{j}\left(\mathcal{E}_{\alpha}\right)_{G} e_{i}\right),
$$

which shows that the Quiver Quot-functor is given as the subfunctor of $\operatorname{Hom}_{S}(*, G)$ which satisfies finitely many pullback equations. It is thus represented by the intersection of the finitely many closed subschemes provided by Proposition 3.8.3. 


\section{Bibliography}

[A09] Arzhantsev, I. V. On the factoriality of Cox rings. (Russian) translated from Mat. Zametki 85 (2009), no. 5, 643-651 Math. Notes 85 (2009), no. $5-6,623-629$.

[ACK07] Álvarez-Cónsul, Luis; King, Alastair. A functorial construction of moduli of sheaves. Invent. Math. 168 (2007), no. 3, 613-666.

[ADHL15] Arzhantsev, Ivan; Derenthal, Ulrich; Hausen, Jürgen; Laface, Antonio. Cox rings. Cambridge Studies in Advanced Mathematics, 144. Cambridge University Press, Cambridge, 2015. viii+530 pp. ISBN: 978-1-107-02462-5.

[Ba11] Bäker, Hendrik. Good quotients of Mori dream spaces. Proc. Amer. Math. Soc. 139 (2011), no. 9, 3135-3139.

[Br14] Brion, Michel. Local properties of algebraic group actions (script). notes for a mini-course at the 3rd Swiss-French workshop on algebraic geometry, Enney, January 27-31 2014, https://algebra.dmi.unibas.ch/blanc/enney2014.html

[BP89] Brion, Michel; Procesi, Claudio. Action d'un tore dans une variété projective. (French) Operator algebras, unitary representations, enveloping algebras, and invariant theory (Paris, 1989), 509-539, Progr. Math., 92, Birkhäuser Boston, Boston, MA, 1990.

[BCL14] Boucksom, Sébastien; Cacciola, Salvatore; Lopez, Angelo Felice. Augmented base loci and restricted volumes on normal varieties. Math. Z. 278 (2014), no. 3-4, 979-985.

[Ca12] Casagrande, Cinzia. Mori Dream Spaces and Fano varieties. expanded notes from a minicourse given at 'Geometrie Algebrique et Geometrie Complexe', CIRM, Marseille, 12-16 March 2012, http://math.univlille1.fr/ serman/GAG2012/MDSGAG2012.pdf.

[Ch08] Chindris, Calin. Notes on GIT-fans for quivers. 2008. arXiv:0805.1440 [math.RT].

[Co95] Cox, David A. The homogeneous coordinate ring of a toric variety. J. Algebraic Geom. 4 (1995), no. 1, 17-50.

[Cr11] Craw, Alastair. Quiver flag varieties and multigraded linear series. Duke Math. J. 156 (2011), no. 3, 469-500.

[CS08] Craw, Alastair; Smith, Gregory G. Projective toric varieties as fine moduli spaces of quiver representations. Amer. J. Math. 130 (2008), no. 6, 1509-1534. 
[DH98] Dolgachev, Igor V.; Hu, Yi. Variation of geometric invariant theory quotients. With an appendix by Nicolas Ressayre. Inst. Hautes Études Sci. Publ. Math. No. 87 (1998), 5-56.

[ER09] Engel, Johannes; Reineke, Markus. Smooth models of quiver moduli. Math. Z. 262 (2009), no. 4, 817-848.

[F98] Fulton, William. Intersection theory. Second edition. Results in Mathematics and Related Areas. 3rd Series. A Series of Modern Surveys in Mathematics, 2. Springer-Verlag, Berlin, 1998. xiv+470 pp. ISBN: 3-540-62046-X; 0-387-98549-2.

[Ga90] Gabriel, Pierre. Des catégories abéliennes. (French) Bull. Soc. Math. France 901962 323-448.

[Gi77] Gieseker, D. On the moduli of vector bundles on an algebraic surface. Ann. of Math. (2) 106 (1977), no. 1, 45-60.

[GW10] Görtz, Ulrich; Wedhorn, Torsten. Algebraic geometry I. Schemes with examples and exercises. Advanced Lectures in Mathematics. Vieweg + Teubner, Wiesbaden, 2010. viii+615 pp. ISBN: 978-3-8348-0676-5.

[GRT16] Greb, Daniel; Ross, Julius; Toma, Matei. Variation of Gieseker moduli spaces via quiver GIT. Geom. Topol. 20 (2016), no. 3, 1539-1610.

[Hal04] Halic, Mihai. Quotients of affine spaces for actions of reductive groups. 2004. arXiv:math/0412278 [math.AG].

[Har77] Hartshorne, Robin. Algebraic geometry. Graduate Texts in Mathematics, No. 52. Springer-Verlag, New York-Heidelberg, 1977. xvi+496 pp. ISBN: 0-387-90244-9.

[Hu92] Hu, Yi. The geometry and topology of quotient varieties of torus actions. Duke Math. J. 68 (1992), no. 1, 151-184.

[HK00] Hu, Yi; Keel, Sean. Mori dream spaces and GIT. Michigan Math. J. 48 (2000), 331-348.

[HL10] Huybrechts, Daniel; Lehn, Manfred. The geometry of moduli spaces of sheaves. Second edition. Cambridge Mathematical Library. Cambridge University Press, Cambridge, 2010. xviii+325 pp. ISBN: 978-0-521-13420-0.

[J11] Jow, Shin-Yao. A Lefschetz hyperplane theorem for Mori dream spaces. Math. Z. 268 (2011), no. 1-2, 197-209.

[K94] King, A. D. Moduli of representations of finite-dimensional algebras. Quart. J. Math. Oxford Ser. (2) 45 (1994), no. 180, 515-530.

[KKV89] Knop, Friedrich; Kraft, Hanspeter; Vust, Thierry. The Picard group of a $G$-variety. Algebraische Transformationsgruppen und Invariantentheorie, 77-87, DMV Sem., 13, Birkhäuser, Basel, 1989.

[KKLV89] Knop, Friedrich; Kraft, Hanspeter; Luna, Domingo; Vust, Thierry. Local properties of algebraic group actions. Algebraische Transformationsgruppen und Invariantentheorie, 63-75, DMV Sem., 13, Birkhäuser, Basel, 1989 . 
[Lan75] Langton, Stacy G. Valuative criteria for families of vector bundles on algebraic varieties. Ann. of Math. (2) 101 (1975), 88-110.

[Laz04] Lazarsfeld, Robert. Positivity in algebraic geometry. I. Classical setting: line bundles and linear series. Results in Mathematics and Related Areas. 3rd Series. A Series of Modern Surveys in Mathematics, 48. SpringerVerlag, Berlin, 2004. xviii+387 pp. ISBN: 3-540-22533-1.

[Lo64] Lojasiewicz, S. Triangulation of semi-analytic sets. Ann. Scuola Norm. Sup. Pisa (3) 181964 449-474.

[LP90] Le Bruyn, Lieven; Procesi, Claudio. Semisimple representations of quivers. Trans. Amer. Math. Soc. 317 (1990), no. 2, 585-598.

[Mar77] Maruyama, Masaki. Moduli of stable sheaves.I. J. Math. Kyoto Univ. 17 (1977), no. 1, 91-126.

[Mas15] Maslovarić, Marcel. Quotients of spectra of almost factorial domains and Mori dream spaces. 2015. arXiv:1508.00539 [math.AG].

[Mat02] Matsuki, Kenji. Introduction to the Mori program. Universitext. SpringerVerlag, New York, 2002. xxiv+478 pp. ISBN: 0-387-98465-8.

[Mi15] Milne, J.S. (Topics in) Algebraic Geometry, Ch. 16: Descent theory. lecture notes, http://www.jmilne.org/math/CourseNotes/ag.html.

[Muk03] Mukai, Shigeru. An introduction to invariants and moduli. Translated from the 1998 and 2000 Japanese editions by W. M. Oxbury. Cambridge Studies in Advanced Mathematics, 81. Cambridge University Press, Cambridge, 2003. $\mathrm{xx}+503$ pp. ISBN: 0-521-80906-1.

[Mum63] Mumford, David. Projective invariants of projective structures and applications. 1963 Proc. Internat. Congr. Mathematicians (Stockholm, 1962) pp. 526-530 Inst. Mittag-Leffler, Djursholm.

[MS17] Maslovarić, Marcel; Seppänen, Henrik. Multi-Gieseker semistability and moduli of quiver sheaves. 2017. arXiv:1710.00371 [math.AG].

[MW97] Matsuki, Kenji; Wentworth, Richard. Mumford-Thaddeus principle on the moduli space of vector bundles on an algebraic surface. Internat. J. Math. 8 (1997), no. 1, 97-148.

[MFK94] Mumford, D.; Fogarty, J.; Kirwan, F. Geometric invariant theory. Third edition. Ergebnisse der Mathematik und ihrer Grenzgebiete (2), 34. Springer-Verlag, Berlin, 1994. xiv+292 pp. ISBN: 3-540-56963-4.

[N05] Nitsure, Nitin. Construction of Hilbert and Quot schemes. Fundamental algebraic geometry, 105-137, Math. Surveys Monogr., 123, Amer. Math. Soc., Providence, RI, 2005.

[O16] Okawa, Shinnosuke. On images of Mori dream spaces. Math. Ann. 364 (2016), no. 3-4, 1315-1342.

[P95] Polito, Marzia. SL(2, C)-quotients de $\left(\mathbf{P}^{1}\right)^{n}$. (French) C. R. Acad. Sci. Paris Sér. I Math. 321 (1995), no. 12, 1577-1582. 
[Rei08] Reineke, Markus. Moduli of representations of quivers. Trends in representation theory of algebras and related topics, 589-637, EMS Ser. Congr. Rep., Eur. Math. Soc., Zürich, 2008.

[Res98] Ressayre, Nicolas. Appendix: An example of thick wall. Inst. Hautes Études Sci. Publ. Math. No. 87 (1998), 53-56.

[R97] Rudakov, Alexei. Stability for an abelian category. J. Algebra 197 (1997), no. $1,231-245$.

[Sch00] Schmitt, Alexander. Walls for Gieseker semistability and the MumfordThaddeus principle for moduli spaces of sheaves over higher dimensional bases. Comment. Math. Helv. 75 (2000), no. 2, 216-231.

[Sch05] Schmitt, Alexander. Moduli for decorated tuples of sheaves and representation spaces for quivers. Proc. Indian Acad. Sci. Math. Sci. 115 (2005), no. $1,15-49$.

[Si94] Simpson, Carlos T. Moduli of representations of the fundamental group of a smooth projective variety. I. Inst. Hautes Études Sci. Publ. Math. No. 79 (1994), 47-129.

[St67] Storch, Uwe. Fastfaktorielle Ringe. (German) Schr. Math. Inst. Univ. Münster No. 361967 iv+42 pp.

[ST16] Seppänen, Henrik, Tsanov, Valdemar V. Unstable loci in flag varieties and variation of quotients. 2016. arXiv:1607.04231v2 [math.RT].

[Ta98] Tarski, Alfred. A decision method for elementary algebra and geometry. Quantifier elimination and cylindrical algebraic decomposition (Linz, 1993), 24-84, Texts Monogr. Symbol. Comput., Springer, Vienna, 1998.

[Th96] Thaddeus, Michael. Geometric invariant theory and flips. J. Amer. Math. Soc. 9 (1996), no. 3, 691-723.

[W94] Weibel, Charles A. An introduction to homological algebra. Cambridge Studies in Advanced Mathematics, 38. Cambridge University Press, Cambridge, 1994. xiv+450 pp. ISBN: 0-521-43500-5; 0-521-55987-1. 


\begin{abstract}
Anhänge
Erklärung gemäß $§ 8.2$

Ich versichere hiermit, dass ich die Dissertation selbstständig und ohne unerlaubte Hilfe angefertigt habe. Ich habe anderweitig keine Promotion beantragt, für die die hier vorgelegte Dissertation oder Teile hiervon eingereicht worden sind.
\end{abstract}

Langenfeld, den 27.11.2017 


\section{Lebenslauf}

\section{Persönliche Daten}

Name Marcel Helmut Maslovarić

Geburtsdatum 29.02.1988

Geburtsort Langenfeld Rhld.

Familienstand ledig

\section{Schulausbildung}

1994-1998 Friedrich-Fröbel-Schule, Grundschule, Langenfeld

1998-2007 Konrad-Adenauer-Gymnasium, Langenfeld

\section{Wehrdienst}

2007-2008 Führungsunterstützungsbataillon 282, Kastellaun

Zentrum Innere Führung, Koblenz

\section{Studium}

2008-2011 Bachelor of Applied Science, Bergische Universität, Wuppertal, in den Hauptfächern Mathematik und Physik

2011-2014 Master of Science, Bergische Universität, Wuppertal im Hauptfach Mathematik, im Nebenfach Physik

seit 2014 Promotionsstudent, Georg-August-Universität, Göttingen Mitglied im Graduiertenkolleg 1493 